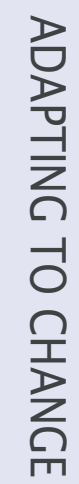

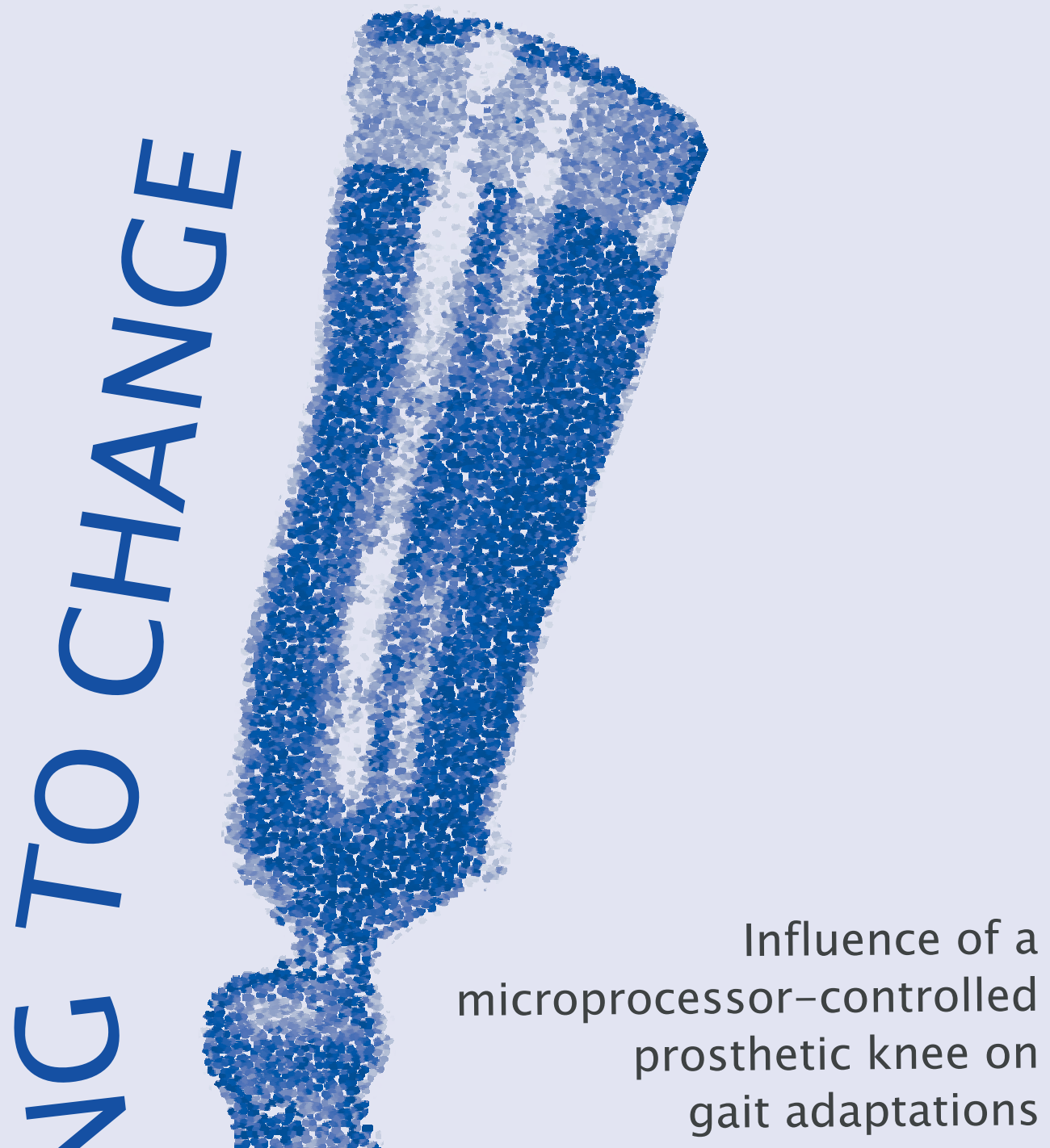

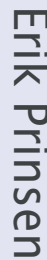

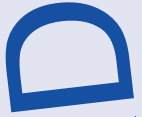

Erik Prinsen

38 


\section{ADAPTING TO CHANGE}

INFLUENCE OF A MICROPROCESSOR-CONTROLLED PROSTHETIC KNEE ON GAIT ADAPTATIONS 
The publication of this thesis was generously supported by:

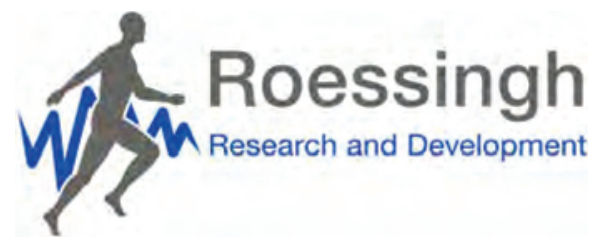

Cover: Jos Spoelstra

Print: Gildeprint - the Netherlands

ISBN: 978-90-365-4206-7

DOI: $10.3990 / 1.9789036542067$

(C)2016, Erik Prinsen, Enschede, the Netherlands

All rights reserved. No part of this book may be reproduced, stored in a retrieval system, or transmitted, in any form or by any means, electronic, mechanical, photocopying, recording, or otherwise, without prior written permission of the holder of the copyright. 


\title{
ADAPTING TO CHANGE \\ INFLUENCE OF A MICROPROCESSOR-CONTROLLED \\ PROSTHETIC KNEE ON GAIT ADAPTATIONS
}

\section{PROEFSCHRIFT}

\author{
ter verkrijging van \\ de graad van doctor aan de Universiteit Twente, \\ op gezag van de rector magnificus, \\ prof. dr. T.T.M. Palstra, \\ volgens besluit van het College voor Promoties \\ in het openbaar te verdedigen \\ op donderdag 8 december 2016 om 14.45 uur
}

door

Erik Christiaan Prinsen

geboren op 15 mei 1983

te Groenlo 
DIT PROEFSCHRIFT IS GOEDGEKEURD DOOR

Prof. dr. J.S. Rietman

Prof. dr. ir. H.F.J.M. Koopman

Dr. M.J. Nederhand 


\section{Voorzitter/Secretaris}

Prof. dr. ir. G.P.M.R. Dewulf

Universiteit Twente

\section{Promotoren}

Prof. dr. J.S. Rietman

Universiteit Twente

Prof. dr. ir. H.F.J.M. Koopman

Universiteit Twente

\section{Co-promotor}

Dr. M.J. Nederhand

Roessingh Research and Development

\section{Overige commissieleden}

Prof. dr. ir. N.J.J. Verdonschot

Universiteit Twente

Prof. dr. V. Evers

Universiteit Twente

Prof. dr. H. Burger

University Rehabilitation Institute, Slovenia

Prof. dr. ir. J. Harlaar

Vrije Universiteit

Prof. dr. F. Nollet

Universiteit van Amsterdam 



\section{INHOUDSOPGAVE}

CHAPTER 1 General introduction.

PAGE 1

CHAPTER 2 Adaptation strategies of the lower extremities of PAGE 16 individuals with a transfemoral or transtibial amputation during level walking: A systematic review.

CHAPTER 3 Comparison of muscle activity patterns of transfemoral PAGE 44 amputees and control subjects during walking.

CHAPTER 4 The influence of a user-adaptive prosthetic knee across PAGE 62 varying walking speeds: A randomized cross-over trial.

CHAPTER 5 Added value of a user-adaptive prosthetic knee in PAGE 78 planned gait initiation: Off to a good start?

CHAPTER 6 The influence of a user-adaptive prosthetic knee on PAGE 92 planned gait termination: A randomized cross-over trial.

CHAPTER 7 Responses of individuals with an amputation to posterior PAGE 108 platform perturbations during walking: influence of a user-adaptive prosthetic knee.

CHAPTER 8 Influence of a user-adaptive prosthetic knee on quality of PAGE 126 life, balance confidence and measures of mobility: A randomized cross-over trial.

CHAPTER 9 General discussion.

PAGE 140

References

PAGE 154

Summary

PAGE 167

Samenvatting

PAGE 171

Dankwoord

PAGE 175

About the author

PAGE 178

Progress range

PAGE 180 

General introduction 


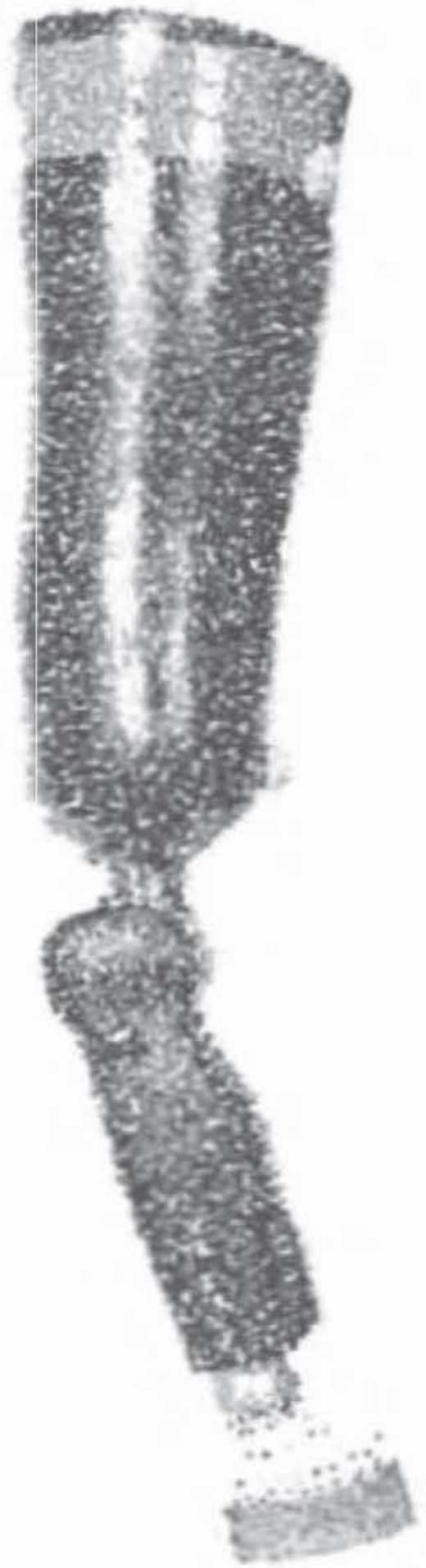




\begin{abstract}
"A person can never be broken. Our built
environment, our technologies, are broken and

disabled. We the people need not accept our

limitations, but can transcend disability through

technological innovation."

Hugh Herr
\end{abstract}

This quote is from the TED talk of Hugh Herr, who is working as an associate professor and director of the Biomechatronics Group in the Media Lab of the Massachusetts Institute of Technology. Next to his research that is aimed at improving rehabilitation and the design of augmentation technology, he has personal experience with prosthetic design as he has a double-sided amputation himself. Being someone with an amputation, he experienced the "inadequacy of the available technology". ${ }^{1}$ Realizing this inadequacy "... was a call to arms, to advance technology for the elimination of my own disability, and ultimately, the disability of others." ${ }^{1}$

Hugh Herr is not alone in this. Over the last decades considerable efforts have been made to improve the functionality of prosthetic components. These advancements have led to the introduction of microprocessor-controlled prosthetic knees (MPK) for individuals with a transfemoral amputation or knee disarticulation. The main focus of this dissertation will be on a specific MPK, the Rheo Knee II, and how its use influences the gait adaptations seen after transfemoral amputation or through-knee amputation. This general introduction will shortly focus on the consequences of a knee disarticulation or transfemoral amputation. This is followed by a description of the different available prosthetic knees and their working mechanisms. In this description, gait adaptations found in individuals with a transfemoral amputation or knee disarticulation will be included. This will be followed by an overview of the evidence for an added value of MPKs and the possibilities for progressing prosthetic knee research. The general introduction will conclude with the objectives and outline of this dissertation.

\title{
KNEE DISARTICULATION AND TRANSFEMORAL AMPUTATION
}

Both a knee disarticulation and a transfemoral amputation result in the loss of the knee and ankle and foot at the amputated side (see Figure 1.1). The difference between the two is that a knee disarticulation is an amputation through the knee and that a transfemoral amputation is performed somewhere between the knee and hip joint. Next to loss of the knee and the ankle and foot, muscle geometry also changes as a consequence of an amputation. Bi-articular muscles spanning the hip and knee become mono-articular hip muscles and mono-articular knee muscles no longer span a joint, which affects the function they can 


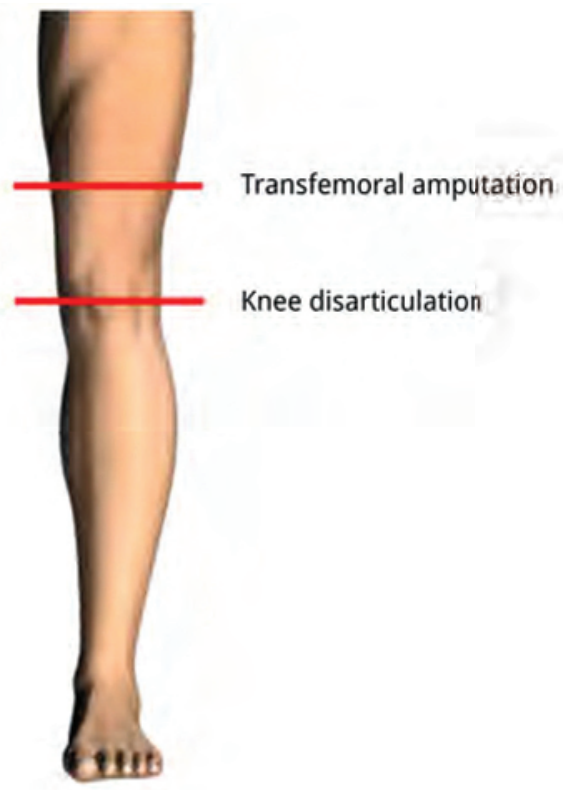

Figure 1.1: Amputation levels

exert. ${ }^{2}$ To substitute for the amputated body part, a prosthesis can be prescribed during the rehabilitation. Generally, a prosthesis consists of: (1) a socket and liner which acts as the interfaces between the prosthesis and the residual leg; (2) a prosthetic knee; (3) a pylon connecting the prosthetic knee to the prosthetic ankle and foot; and (4) a prosthetic ankle and foot. As this thesis focuses on the comparison of different prosthetic knee units, the main focus of this introduction section will be on prosthetic knees. The role of the prosthetic ankle is also shortly discussed.

\section{PROSTHETIC ANKLE-FOOT UNITS}

Prosthetic ankle-foot units are designed to substitute parts of the functions of the ankle and foot complex, which are: (1) shock absorption, (2) weight-bearing stability, and (3) progression. ${ }^{3}$ Prosthetic ankle-foot units can be rigid (e.g. the SACH foot), store and release energy (e.g. the Flex foot), or can include a motor that is able to generate energy (e.g. the BiOM ankle). In daily clinical practice, motorized prosthetic ankle-foot units are not prescribed often. Rigid and energy storing and releasing prosthetic ankle-foot units are limited in fulfilling the functional roles of the ankle-foot complex. The lack of active ankle control leads to problems in predominantly weight-bearing stability and progression. 


\section{PROSTHETIC KNEE UNITS}

There are a large number of prosthetic knee units commercially available, which have different features. To provide standardization in the description of prosthetic knee units, the ISO norm 13405-2:2015 presents a classification tree based on four features. These are: (1) motions (flexion/extension and/or axial rotation), (2) axis of rotation (monocentric/polycentric), (3) activation and control mechanism, and (4) transition between stance and swing phase. ${ }^{4}$

In daily clinical practice and in the scientific community the activation and control mechanism of prosthetic knees is used to further classify prosthetic knees. Control of prosthetic knees can be achieved by friction, pneumatic or hydraulic means. In turn, this control can be adjustable, adaptable or auto-adaptable. In this context, adjustable means that the features of prosthetic components can be changed before use by the manufacturer, prosthetist, or user. ${ }^{5}$ Adaptable indicates prosthetic components whose features can be changed by the user to make it suitable for different situations. ${ }^{5}$ Auto-adaptive components are prosthetic components whose features change automatically in response to varying situations in daily life. $^{5}$

Based on the activation and control mechanism prosthetic knees can be divided into:

1. Non-microprocessor-controlled prosthetic knees (NMPKs)

2. Microprocessor-controlled prosthetic knees (MPKs)

3. Powered prosthetic knees

These categories can be distinguished from one another based on two characteristics: (1) adjustable/adaptable vs auto-adaptive control and (2) the absence or presence of actuation. NMPKs are adjustable/adaptable whereas MPKs and powered prosthetic knees are auto-adaptive. In turn, NMPKs and MPKs both have no actuation. This means that these types of prosthetic knees can only dissipate energy. Powered prosthetic knee do have actuation, which means that these prosthetic knees can generate energy. Powered prosthetic components are not commonly prescribed in daily practice at the moment, mostly because of their high price. Powered prosthetic knees fall outside the scope of this thesis and will, therefore, not be further discussed in this chapter. In the general discussion (chapter 9), powered prosthetic knees will be discussed in more details.

\section{Non-microprocessor-controlled prosthetic knees}

In Figure 1.2 several examples of NMPKs are shown. As stated before, NMPKs can have an adjustable and/or adaptable control. One example of adaptable control is that there are prosthetic knees that can be manually locked or unlocked. One example of an adjustable control is the level of resistance against knee flexion (knee damping) and assist of knee extension which can be set by the prosthetist or the user before use. The fact that control can 

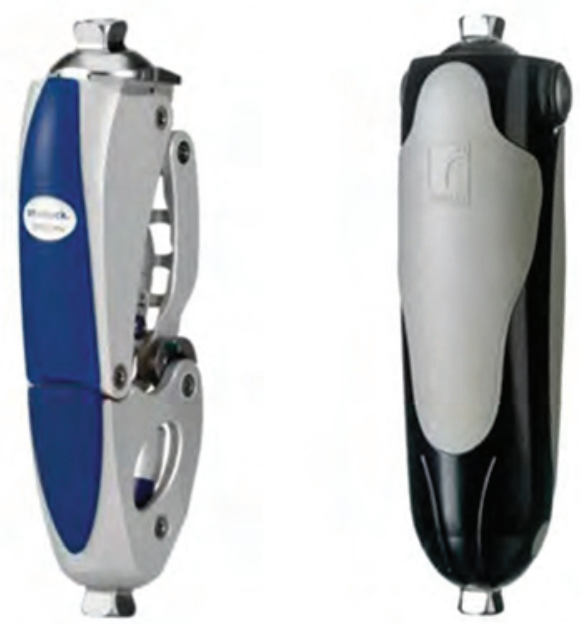

Figure 1.2: Examples of NMPKs; Left: Otto Bock 3R60; Right: Össur Mauch SNS

only be changed before use, directly shows the limitations of NMPKs with adjustable control. Faster or slower walking and negotiating stairs or ramps requires different amounts of knee damping. As NMPKs are not capable of changing their knee damping while performing these activities of daily living, knee damping is not optimal. This in turn, might require additional compensations of the individual with an amputation. Because of their fixed settings, NMPKs are limited in fulfilling the functional tasks of a physiological knee.

The first role of the knee is shock absorption during the first phase of double limb support. ${ }^{3}$ By allowing knee flexion during the loading response, the knee absorbs the energy that is associated with the weight transfer during double limb support. 3,6 In most commercially available NMPKs early stance knee flexion is not possible. ${ }^{7-10}$ Allowing early stance prosthetic knee flexion would require high knee damping. This high level of damping would restrict prosthetic knee flexion later on in the gait cycle when the knee has to be sufficiently flexed for forward progression of the swing leg. As restricted knee flexion during swing is undesirable, knee damping is not set at the levels that are required to allow early stance knee flexion. There are NMPKs available in which stance and swing phase control can be adjusted separately from one another. In, for example, the Mauch SNS, knee damping during the stance phase can be set at a level that would allow early stance prosthetic knee flexion and a different level of knee damping during the swing phase allowing sufficient knee flexion during swing. However, previous studies showed that individuals with an amputation exhibit no early stance prosthetic knee flexion while walking with the Mauch SNS. ${ }^{11,12}$ The reason of the lack of early stance prosthetic knee flexion while walking with the Mauch SNS is not known. A possible explanation could be that the knee has to be moved towards extension during mid stance. In individuals without an amputation, this is achieved by a brief 
concentric contraction of the knee extensors. ${ }^{13}$ Because the quadriceps muscle is cut during the amputation, this mechanism is impaired in individuals with a transfemoral amputation or knee disarticulation. In these individuals, moving the knee towards extension can only be achieved by hip extension through gluteal muscle action. Because of the short lever arm of the gluteus maximus to the joint center of rotation, this would require a high joint torque and, thus, could be energy inefficient. Whether this explanation is viable and applicable to individuals with an amputation is yet unknown.

The second role of the knee is to provide stability throughout the stance phase. ${ }^{3}$ To increase stability, prosthetic knees with polycentric knee axes have been developed. Polycentric knee units are free to flex and extend but facilitate knee joint stabilization because their instantaneous axis of rotation moves posteriorly as the prosthetic knee joint moves towards extension. ${ }^{4} \mathrm{~A}$ more posterior position of the instantaneous axis of rotation increases the likelihood that the ground reaction force vector is aligned anterior to the knee. This anterior position of the ground reaction force with respect to the knee joint center creates a knee extension moment on the prosthetic knee leading to a stable situation. Despite the development of prosthetic knees with increased stance stability, falling is still a prevalent problem amongst individuals with an amputation. The results of a survey published in 2001 indicated that $66 \%$ of the participants with a transfemoral amputation fell at least once in the last 12 months. $^{14}$

The third and final role of the knee is to achieve sufficient knee flexion during the swing phase in order to progress the swing leg forward. ${ }^{3}$ Knee damping in adjustable NMPKs can be set to be optimal at preferred walking speed. However, at lower or faster walking speed this knee damping is non-optimal. At lower walking speeds, knee damping should be lower and at higher walking speeds, knee damping should be higher. Previous trials studying NMPKs across different walking speeds indeed found that peak prosthetic knee flexion during swing increases with walking speed. ${ }^{10,15}$

\section{Gait adaptations associated with the use of NMPKs}

The fact that NMPKs are limited in fulfilling the functional roles of a physiological knee means that individuals with an amputation have to adapt their gait pattern to be able to safely walk with a NMPK.

One of the gait adaptations that is seen in individuals with a transfemoral amputation or knee disarticulation is decreased loading of the prosthetic leg and increased loading of the intact leg. ${ }^{12,16}$ It is thought that this is related to the limited prosthetic knee flexion during stance, which causes a higher vertical position of the center of mass. ${ }^{17,18}$ This higher position means that the center of mass covers a larger vertical trajectory during the loading response of the intact leg, potentially leading to a higher loading of the intact leg.

The limited amount of damping NMPKs can provide during early stance and associated chance of knee buckling also leads to gait alterations. Individuals with a transfemoral amputation or knee disarticulation show increased hip extensor activity of the amputated 
leg during (early) stance. ${ }^{2}$ Through the closed kinetic chain increased hip extensor activity assists in keeping the prosthetic knee extended. Second, the double support phase after initial contact of the prosthetic leg is increased when compared to the other double support phase.

The fact that NMPKs might lead to non-optimal knee kinematics also might be of influence on the gait pattern. Too little prosthetic knee flexion during swing increases the risk of toe dragging. It is thought that this might lead to intact ankle vaulting which can be defined as "a premature midstance plantar flexion of the sound limb which assist toe clearance of the prosthetic limb by lifting the body". ${ }^{19}$ Vaulting leads to a relative increase in leg length of the intact leg which decreases the chance of toe dragging of the prosthetic leg. The causal link between vaulting and reduced prosthetic knee flexion during swing, however, has not been established yet in individuals with a transfemoral amputation or knee disarticulation.

Finally, there are several gait adaptions seen in individuals with an transfemoral amputation or knee disarticulation that cannot be linked to one specific functional role of the knee, but seem to be the results of a combination of factors. These include a reduced preferred walking speed, ${ }^{10,12,16}$ reduced duration of single limb support on the prosthetic leg, ${ }^{10}$ and increased duration of the prosthetic swing phase. ${ }^{10}$

\section{Microprocessor-controlled prosthetic knees}

Relating back to the quote of Hugh Herr at the start of this chapter, the fixed pre-set damping properties of NMPKs are one of the "broken and disabled" characteristics of these prosthetic knees. From the 1970s onwards, research groups have focused on advancing the design of NMPKs which have led to the development of MPKs. The first MPK to be commercially available was the Intelligent Prosthesis (now known as the SmartIP) which was released by Blatchford in 1993. This was followed by the release of the C-Leg, which was introduced in 1997 by Otto Bock. At the Massachusetts Institute of Technoloy Hugh Herr worked on his own version of a MPK, details of which were published in $2003 .{ }^{7}$ This MPK went on to become known as the Rheo Knee which was released by Össur in 2004.

MPKs have auto-adaptive control, which means that knee damping changes automatically in response to varying walking situations. To be able to do so, MPKs incorporate sensors measuring variables such as knee angle, knee angular velocity, knee moment and force applied on the prosthesis. Based on the information of these sensors, a control algorithm adapts knee damping to the desired level. These prosthetic knees claim to provide early stance prosthetic knee flexion, increase stance stability during stance, and provide optimal knee damping during swing irrespective of walking speed. ${ }^{7,20}$ They, thus, should be able to fulfill the roles of the knee to a larger extent than NMPKs. Figure 1.3 shows several MPKs that are commercially available.

The question whether MPKs are beneficial for individuals with an amputation has been subject of several studies. In these studies the use of a MPK is usually compared to the use of a NMPK. While there are several MPKs on the market, research has predominantly 

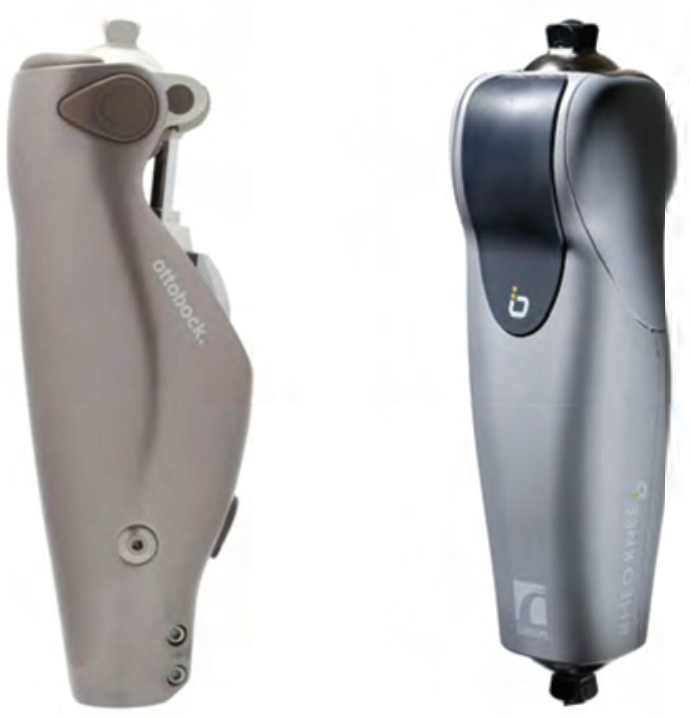

Figure 1.3: Examples of MPKs; Left: Otto Bock C-Leg; Right: Össur Rheo Knee

focused on the SmartIP (formerly known as the Intelligent Prosthesis), the C-Leg, the C-Leg Compact, the Rheo Knee, and the Genium. While these prosthetic knees all have autoadaptive control mechanism, there are some distinct differences between them. In Table 1.1 these differences are described.

Because this thesis focuses on the Rheo Knee II, this prosthetic knee is described into more detail. The Rheo Knee incorporates a magnetorheological fluid, which is a carrier oil in which magnetic particles are dispersed. Based on the information of the sensors, electromagnets control the magnetic field within a magnetorheological fluid. ${ }^{7}$ The magnetical particles in the magnetorheological fluid form torque-producing chains in response to the applied magnetic field. ${ }^{7}$ The Rheo Knee, thus, can control knee damping by controlling the magnetic field. Next to the sensors, the Rheo Knee also included artificial intelligence known as the Dynamic Learning Matrix Algorithm. ${ }^{21}$ This aim of this algorithm is to learn the individual's walking style. ${ }^{21}$ This should enable parameters to constantly change over time, instead of adapting parameters within pre-set and limited parameters. ${ }^{21}$ This feature is called user-adaptive control. ${ }^{7}$

\section{EVIDENCE FOR MPKS}

In recent years, multiple systematic reviews have been performed that aim to collect and appraise the literature comparing MPKs to NMPKs in individuals with a transfemoral amputa- 
Table 1.1: Differences between microprocessor-controlled prosthetic knees

\begin{tabular}{|c|c|c|c|}
\hline Name & $\begin{array}{l}\text { Gait phase of auto-adaptive } \\
\text { control }\end{array}$ & Type of control & Sensors \\
\hline SmartIP & Swing phase & Pneumatic & $\begin{array}{l}\text { Proximity switch detecting step } \\
\text { time }\end{array}$ \\
\hline C-Leg 4 & Stance and swing phase & Hydraulic & $\begin{array}{l}\text { Inertial magnetic units, load } \\
\text { cells, knee angle sensor }\end{array}$ \\
\hline C-Leg compact & Stance phase & Hydraulic & $\begin{array}{l}\text { Strain gauges, knee angle sensor, } \\
\text { knee angular velocity sensor }\end{array}$ \\
\hline Rheo Knee II & Stance and swing phase & Magnetorheological & $\begin{array}{l}\text { Load cells, knee angle sensor, } \\
\text { knee angular velocity }\end{array}$ \\
\hline Genium & Stance and swing phase & Hydraulic & $\begin{array}{l}\text { Gyroscope, inertial magnetic } \\
\text { units, knee angle sensor, knee } \\
\text { moment sensor, load cells }\end{array}$ \\
\hline
\end{tabular}

tion and knee disarticulation. ${ }^{22-24}$ These reviews all have a slightly different aim: Highsmith et al. ${ }^{23}$ solely focused on the C-Leg in their review, Kannenberg et al. ${ }^{24}$ focus on limited community ambulators only, and Sawers and Hafner ${ }^{22}$ include all available literature. Because Sawers and Hafner appear to have written the review with the broadest scope, this review is taken as starting point for the presentation of the evidence for MPKs. Based on the results of included studies, Sawers and Hafner drew empirical evidence statements, which are presented below. The results of trials published after the literature search of Sawers and Hafner was completed (October 2009) that could be combined with one of the empirical evidence statement are added to results of this review. Because this dissertation focuses on the Rheo Knee, only the results relating to stance and swing phase MPKs are presented. Next to this, only the results of gait mechanics, environmental obstacle negotiation, safety, preference and satisfaction, and health and quality of life will be presented here as these outcomes will be the focus of this thesis.

\section{Gait mechanics}

There is a low level of evidence that the use of stance and swing MPKs result in an increased preferred walking speed, ${ }^{11,12,25,26}$ equivalent spatiotemporal symmetry, ${ }^{11,12,27,28}$ and increased prosthetic knee moment ${ }^{11,12,29}$ when compared with the use of NMPKs. In addition, there is insufficient evidence that the use of stance and swing MPKs result in equivalent peak prosthetic knee flexion angle during early stance at preferred walking speed ${ }^{7,12,29}$ and equivalent prosthetic power in late stance ${ }^{11,12}$ when compared to the use of NMPKs.

Three studies have been found published after the search strategy of Sawers and Hafner was completed. ${ }^{29-31}$ The results of the trials of Kaufman et al. ${ }^{29}$ and Mâaref et al. ${ }^{30}$ cannot be combined with results of other trials and are therefore omitted. Schaarschmidt et al. ${ }^{31}$ found no differences in spatiotemporal symmetry between walking with a MPK and a NMPK, further strengthening this empirical evidence statement. 


\section{Environmental obstacle negotiation}

There is a low level of evidence that the use of stance and swing MPKs results in increased walking speed on uneven terrain ${ }^{26,27,32,33}$ and in an improved gait pattern during stair descent ${ }^{26,27,32}$ when compared with the use of NMPKs.

Two new publications comparing the use of a MPK to a NMPK while walking an obstacle course were found. Hafner et al. ${ }^{34}$ found no differences in walking speed, whereas Meier et al. ${ }^{35}$ found an increase in walking speed while using a MPK to navigate an obstacle course. These results, thus, are both in conflict and in line with the results of the studies included by Sawers and Hafner. ${ }^{22}$

Three studies have been published after October 2009 that compared the use of MPKs and NMPKs while descending a slope. ${ }^{34,36,37}$ The results of these studies could be combined with one previously conducted trial ${ }^{27}$ which was omitted by Sawers and Hafner ${ }^{22}$ as it was the only available study that used the Hill Assessment Index at that time. There is ambiguity in results when looking at qualitative aspects of slope descent (e.g. use of hand rails, use of walking aid). Both an increase in Hill Assessment Index (HAI) score when walking with a MPK $^{36,37}$ as no differences in HAI score between MPK and NMPK use ${ }^{27}$ have been reported. A higher HAI score indicates a higher qualitative performance, such as no use of hand rail or walking aids. There is also ambiguity when looking at the time needed to descent a slope. Both a decrease 27,36 as an increase in time ${ }^{34}$ have been reported while using a MPK compared to the use of a NMPK.

\section{Safety}

There is a low level of evidence that the use of stance and swing phase MPKs result in decreased number of subject-reported stumbles and falls when compared with the use of NMPKs. ${ }^{26-28,32}$ Next to this, there is insufficient evidence that the use of stance and swing phase MPKs results in decrease in subject-reported frustration with falling when compared with the use of NMPKs. ${ }^{27,32}$ Finally, there is moderate evidence that the use of stance and swing MPKs result in increased subject-reported confidence while walking when compared with the use of NMPKs. ${ }^{27,32,38,39}$

Two trials ${ }^{34,36}$ have been published studying aspects of safety after the search strategy of the review of Sawers and Hafner was completed. The trial of Hafner et al. ${ }^{34}$ found no statistical significant difference in subject-reported confidence between the use of a MPK or a NMPK. This result is in contrast to the results of the included studies by Sawers and Hafner. ${ }^{22}$ The publication by Highsmith et al. ${ }^{36}$ presents some additional calculations based on previously published results. ${ }^{29}$ Because these results could not be combined with the results of other studies, the publication of Highsmith et al. is omitted.

\section{Preference and satisfaction}

There is a low level of evidence that the use of swing and stance MPKs results in increased subject-reported preference ${ }^{25-28}$ and satisfaction ${ }^{26,27}$ when compared with using NMPKs. 
No new trials studying preference or satisfaction were identified.

\section{Health and quality of life}

There is a moderate level of evidence that the use of swing and stance MPKs results in equivalent self-reported general health ${ }^{27,40}$ and well-being ${ }^{27,32,41}$ when compared with the use of NMPKs. In addition, there is a moderate level of evidence that the use of swing and stance MPKs results in increased quality adjusted life years when compared with the use of NMPKs. ${ }^{42,43}$

One new publication was identified that studied several aspects of health and quality of life. ${ }^{34}$ The authors found no differences in general health between the use of MPK and NMPK which is in line with the results of the studies included by Sawers and Hafner. ${ }^{22}$

\section{Conclusion}

In conclusion, while a considerable number of efforts have been made to study the potential added value of a MPK when compared to the use of a NMPK there is substantial ambiguity in the published results. Comparison of more objective to more subjective outcome parameters seem to indicate that the users' perception of the added value of a MPK is higher than the objective outcome parameters seem to be able to explain. In addition, the added value of MPK seems to be more pronounced in activities outside of level walking at preferred walking speed such as stair and slope walking.

\section{POSSIBILITIES FOR PROGRESSING PROSTHETIC KNEE RESEARCH}

The relatively low incidence of transfemoral amputation and knee disarticulation combined with the relatively high mortality rate in individuals in which the amputation was caused by vascular problems, which is the main reason of amputation in the western world, make it hard to have large study populations. This increases the chances that studies are underpowered making it harder to find statistical significant differences as these differences need to be larger to be detected. One solution for this problem could be a meta-analysis of earlier conducted trials in which the results of comparable outcome parameters are pooled. In case of data pooling, the study population is increased, possibly increasing statistical power and the chance to detect statistically significant differences.

In addition, while there is a substantial body of knowledge regarding spatiotemporal, kinematic and kinetic variables of the walking pattern of individuals with an amputation, muscle activation patterns have been scarcely studied. More in-depth insight into muscle activity during walking of individuals with a knee disarticulation or transfemoral amputation could increase our understanding of the possible changes in motor programs used for locomotion.

Next to this, the majority of studies comparing MPKs to NMPKs have focused of the C- 
Leg, whereas the Rheo Knee has received considerably less attention. 7,11,34,44,45 As discussed before, the Rheo Knee has a different control algorithm and mechanism when compared to the C-Leg. Therefore, there might be a difference in effect between the Rheo Knee and the C-Leg. The trial of Johansson et al., indeed, suggest that there are some differences between the Rheo Knee vs Mauch SNS on one side, and the C-Leg and Mauch SNS on the other side. ${ }^{11}$ This indicates the necessity to evaluate the effectiveness of the Rheo Knee when compared to NMPKs.

Finally, the effect of MPKs has predominantly been evaluated during walking at preferred walking speed and ramp and/or stair negotiation. The effect of a MPK across different walking speeds has been scarcely studied. In addition, the effect of a MPK during the transitional stages of gait has not been studied yet. Finally, there have been no attempts to quantify the influence of a MPK on the responses on balance perturbations during walking.

\section{GENERAL AIM AND RESEARCH QUESTIONS}

Based on the possibilities for progressing microprocessor-controlled prosthetic knee research, the overarching aims of this thesis are to increase our understanding about gait adaptations seen after amputation and investigate how a user-adaptive prosthetic knee influences these gait adaptations. Based on the general aim, this thesis aims to answer the following research questions:

1. What compensations in terms of joint work and power can be seen at the hip and knee of the amputated leg and the joints of the intact leg of individuals with a transtibial amputation. In addition, what compensations in terms of joint work and power can be seen at the hip of the amputated leg and the joints of the intact leg of individuals with a transfemoral amputation or knee disarticulation?

2. What are differences in muscle activation patterns during walking of individuals with a transfemoral amputation or knee disarticulation when compared to individuals without an amputation?

3. What is the influence of the Rheo Knee II on gait adaptations seen during level walking at varying walking speeds, gait initiation, gait termination, and responses to platform perturbation during walking?

4. What is the influence of the Rheo Knee II on prosthetis-related quality of life, functional status, and balance confidence? 


\section{General OUtLine}

In chapter 2 we present the systematic review and meta-analysis we performed synthesizing the available joint power and joint work data of level walking of individuals with a transitibial or transfemoral amputation. Because only few studies quantified muscle activation patterns of prosthetic gait we measured muscle activity of upper leg muscles in six individuals with a transfemoral amputation or knee disarticulation. We compared these data with muscle activity measured in five individuals without an amputation. The results are presented in chapter 3.

In chapter 4-8 we present the results of the randomized cross-over trial we performed comparing the Rheo Knee II to NMPKs. Ten individuals with a transfemoral amputation and ten individuals without an amputation were included in this trial. In chapter $\mathbf{4}$ we present the results of our level walking experiments in which participants walked at three walking speeds. In the data analysis we specifically emphasized on prosthetic knee kinematics during stance and swing and compensatory movements associated with non-optimal prosthetic knee kinematics. In chapter 5 and $\mathbf{6}$ the results of, respectively, our gait initiation and gait termination experiments are presented. In the data analysis we primarly focused on the role of the prosthetic leg in generating forces (gait initiation) and absorbing forces (gait termination). Chapter 7 presents the results of our evoked balance perturbations experiments. We quantified the margins of stability and variables that influence the margins of stability. We present the influence of the Rheo Knee II on prosthesis-related quality of life, balance confidence, and functional status in chapter 8. Finally, in chapter 9 we discuss the results of the presented studies and possibilities of future research in the field of user-adaptive prosthetic knees and other prosthetic components in general. 

Adaptation strategies of the lower extremities of individuals with a transfemoral or transtibial amputation during level walking: A systematic review

Prinsen EC, Nederhand MJ, Rietman JS. Arch Phys Med Rehabil 2011;92:1311-25. 


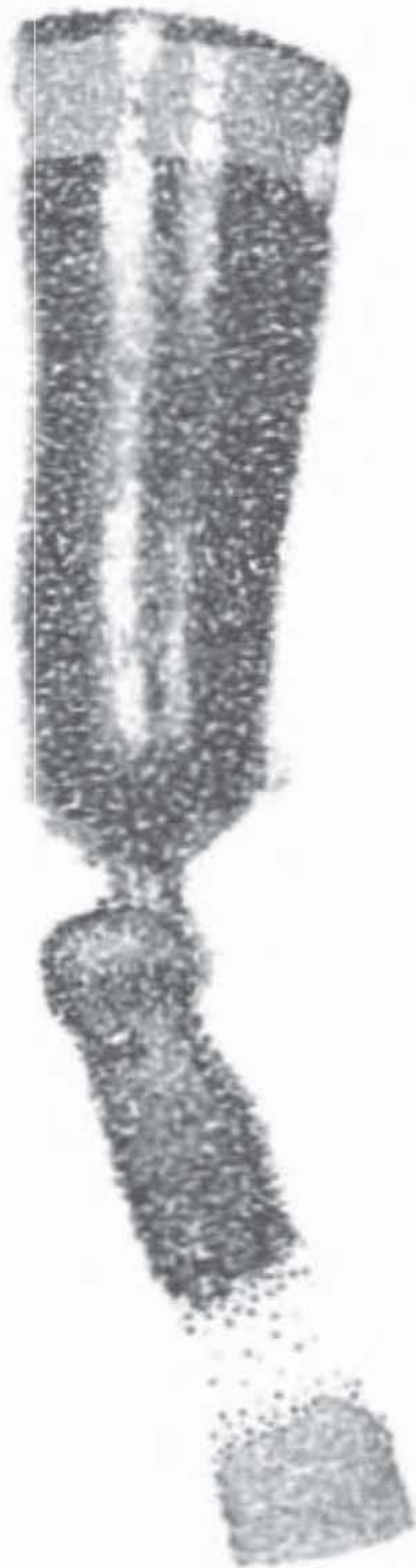




\section{Abstract}

Objective To describe adaptation strategies in terms of joint power or work in the amputated and intact leg of patients with a transtibial (TT) or transfemoral (TF) amputation.

Data sources MEDLINE, CINAHL, Physiotherapy Evidence Database, Embase, and the Cochrane Register of Controlled Trials were searched. Studies were collected up to November 1 , 2010. Reference lists were additionally scrutinized.

Study selection Studies were included when they presented joint power or work and compared (1) the amputated and intact legs, (2) the amputated leg and a referent leg, or (3) the intact leg and a referent leg. Eligibility was independently assessed by 2 reviewers. A total of 13 articles were identified.

Data extraction Data extraction was performed using standardized forms of the Cochrane Collaboration. Methodologic quality was independently assessed using the Downs and Black instrument by 2 reviewers. The possibility of data pooling was examined. Significant differences found in studies that could not be pooled are also presented.

Data synthesis Significant results $(P<.05)$. For work TT, for the concentric work total stance phase knee, the amputated was less than the intact/referent side, and the referent was less than the intact side. For the eccentric knee extensor (K1) phase, the amputated was less than the intact side, and the intact was greater than the referent side. For the concentric knee extensor (K2) phase, the amputated/referent was less than the intact side. For the concentric work total stance phase hip, the amputated/intact was greater than the referent side. For the concentric hip extensor ( $\mathrm{H} 1)$ phase, the amputated/intact was greater than the referent side. For power TT, for the peak power generation stance phase knee, the amputated was less than the referent side. For peak power generation swing phase knee, the amputated was less than the referent side. For the eccentric knee flexor (K4) phase, the amputated was less than the intact side. For the eccentric hip flexor ( $\mathrm{H} 2)$ phase, the amputated was greater than the intact side. For work TF, for the concentric plantar flexor (A2) phase, the referent was less than the intact side. For the $\mathrm{H} 1$ phase, the referent was less than the intact side. For the $\mathrm{H} 2$ phase, the amputated was greater than the intact/referent side, and the referent was greater than the intact side. For power TF, for the K2 phase, the referent was less than the intact side. Sensitivity analysis did not alter the conclusions.

Conclusions Adaptations were seen in the amputated and intact legs. TT and TF use remarkably similar adaptation strategies at the level of the hip to compensate for the loss of plantar flexion power and facilitate forward progression. At the knee level, adaptations differed between TT and TF. 


\section{INTRODUCTION}

Walking is highly dependent on dynamic interactions between sensory afferents and the central motor program for locomotion. ${ }^{46}$ Because an amputation leads to the loss of sensorimotor function of a leg, these skills are challenged. During the period of rehabilitation, a person with an amputation learns to compensate for the deterioration of these skills by adaptation strategies in both the intact leg and the remaining stump.

There are numerous ways to describe the gait pattern of people with an amputation, such as using temporal-spatial, kinematic, and/or kinetic variables. Kinetic variables are used to describe the forces acting across joints and include joint moment, power, and work. Joint power is defined as the product of the joint moment and joint angular velocity ${ }^{13}$ and gives insight into the net power produced or absorbed by the uniarticular and biarticular musculotendon complexes. ${ }^{47}$ Power studies allow quantification of the contribution of the musculotendon complex to the observed motion of body segments. ${ }^{47}$ Joint work is computed as the time integral of joint power. ${ }^{48}$ Power and work can have positive and negative values. By convention, positive values represent energy generation, and negative values represent energy absorption. Energy generation is the result of concentric muscle action, energy absorption the result of eccentric muscle action. ${ }^{13}$

Winter ${ }^{13}$ studied the gait of able-bodied people and categorized the power profile into different phases. The ankle has 2 major phases: (1) the eccentric plantar flexor (A1) phase as the tibia rotates forward over the foot flat, and (2) the concentric plantar flexor (A2) phase (push-off) as the foot plantar flexes prior to swing phase. The knee has 4 major phases: (1) the eccentric knee extensor (K1) phase as the knee flexes during weight acceptance, (2) the concentric knee extensor (K2) phase as the knee extends during midstance, (3) the eccentric knee extensor (K3) phase during push-off as the knee flexes, and (4) the eccentric knee flexor (K4) phase as the knee extends at the end of swing. The hip has 3 major phases: (1) the concentric hip extensor $(\mathrm{H} 1)$ phase as the hip extends during weight acceptance, (2) the eccentric hip flexor $(\mathrm{H} 2)$ phase to decelerate the backward rotating thigh, and (3) the concentric hip flexor (H3) phase as the hip flexes prior to swing phase. These phases are also applicable on the intact joints of amputees. ${ }^{49}$ The phases are less applicable on the prosthetic joints because most prosthetic components are passive and therefore solely capable of absorbing energy.

In the able-bodied, the generation of energy during the A2 phase plays an important role in the forward progression of the body, ${ }^{13,50-53}$ the acceleration of the leg into swing, ${ }^{53,54}$ and the generation of knee flexion during swing. ${ }^{55}$ Previous research has shown that in persons with a transtibial (TT) or transfemoral (TF) amputation, significantly less power is generated during the A2 phase in the amputated leg compared with the ankle of an ablebodied person. ${ }^{48,56-58}$ Because the generation of energy of the ankle is decreased, persons with TT and TF amputation must adapt the motor strategies used for forward progression of the body, acceleration of the leg, and achievement of sufficient knee flexion during the 
swing phase.

In TF amputation, there is an additional loss of sensorimotor function of the knee. To our knowledge, no studies have been published describing the generated and absorbed energy of the prosthetic knee of TF amputation. However, reduced knee moments and knee range of motion of the prosthetic knee have been described, ${ }^{59}$ thereby affecting the gait pattern of TF amputees.

Insight in the gait adaptation of TT and TF amputation could help improving prosthetic devices and form the basis for rehabilitation programs. To fulfill these objectives, numerous studies have been performed studying power and work performance of both the amputated and intact legs. These studies generally use small study populations, making it difficult to reach sufficient statistical power. Therefore, combining the results of multiple studies would be interesting. This will increase the overall study population and thereby, possibly, the statistical power.

We hypothesized that the required adaptation strategies needed for successful ambulation would have an effect on joint power and work. Furthermore, we hypothesized that these adaptations will take place in both the intact leg and the amputated leg. The aim of this systematic review is to describe the adaptation strategies in both the intact leg and the amputated leg in TT and TF amputation in terms of joint power or work compared with able-bodied or normative data.

\section{Methods}

\section{Literature search}

We conducted a computerized literature search in MEDLINE (1950 to November 2010), Embase (<1950 to November 2010), CINAHL (1966 to November 2010), Physiotherapy Evidence Database (1929 to November 2010), and the Cochrane Central Register of Controlled Trials (Cochrane Library 2010 issue 4). The search strategy used in MEDLINE was based on the following Medical Subject Headings terms: amputation, amputees, artificial limbs, walking, gait, locomotion, biomechanics, kinetics, and muscle. We adapted the search strategy to fit the different databases. In addition, we examined the reference lists of potentially relevant articles.

\section{Selection criteria}

There were no restrictions on study design because most trials studying gait use an observational study design, which is already considered a low level of evidence. If restrictions on study design were applied, most eligible studies would have been excluded. We included studies in which the participants had TT or TF amputation and were able to walk without assistance devices. Outcome measures had to include joint power or work obtained in level walking. Studies were eligible when comparisons were made between (1) an amputated leg 
and the contralateral intact leg, (2) the amputated leg and a referent leg (leg of an ablebodied person) or normative data, or (3) the intact leg of an amputee and a referent leg or normative data. We placed no restrictions on used feet and/or prosthesis. Finally, we excluded studies not published in English, Dutch, or German.

\section{Selection of studies}

The titles and abstracts of the studies identified in the literature search were independently scrutinized by E.C.P. and M.J.N. on potential eligibility for inclusion. If the title and abstract provided inconclusive information, we retrieved the full-text version to decide on inclusion.

If the studies met the inclusion criteria but failed to report information needed for data synthesis, we contacted the primary author. If the required information could be provided, the study was included. When the primary author could not provide the information or when there was no response on the request, the study was excluded.

\section{Categorization of studies}

We categorized studies according to amputation level into TT and TF amputation, and according to presented outcome measures into either joint power or work. Furthermore, we investigated the possibility to combine multiple trials studying a specific type of foot and/or prosthesis. We analyzed each category separately. When studies used different walking speeds, we used the data obtained at the walking speed that most closely resembled the walking speed of the other trials in the category in the analysis.

Based on an exploratory search, it was expected that study results would include both peak values with ambiguity on timing and peak values presented according to the phases of Winter. ${ }^{13}$ When the timing of the peak value was unclear, we checked the results section for a graphic presentation of the power profile. If this was available, the graphic presentation was studied to determine whether the peak value corresponded with one of the phases described by Winter. ${ }^{13}$ In the case of correspondence, we analyzed the peak values as being a peak of a phase described by Winter. ${ }^{13}$ In the case of non-correspondence or when no graphic presentation was provided, we used the peak value in the analysis and ambiguity on timing of the peak value remained.

\section{Methodologic quality and data extraction}

We assessed methodologic quality of included studies with the instrument developed by Downs and Black. ${ }^{60}$ The checklist includes 27 items in 5 subscales: reporting $(n=10)$, external validity $(n=3)$, internal validity bias $(n=7)$, internal validity confounding $(n=6)$, and power $(n=1)$. The maximal score is 32 , with a higher score representing a better methodology. The test retest reliability $(r=.89)$, interrater reliability $(r=.75)$, and internal consistency (Kuder-Richardson formula $20=.89$ ) of the checklist is good. ${ }^{60}$ The included studies were scored independently by 2 reviewers (E.C.P. and an independent reviewer). In the case of disagreement, consensus was sought, but when disagreement persisted, a third reviewer 
(M.J.N.) made a final decision.

We used standardized forms developed by the Cochrane Collaboration for the data extraction. ${ }^{61}$ Characteristics of trials and participants, relevant outcomes, and references to potentially relevant studies not identified during the literature search were recorded.

\section{Data synthesis}

We studied the results of the studies within each category to investigate the possibility of data pooling. When this was possible, mean difference with $95 \%$ confidence interval, heterogeneity, and confounding were calculated using Review Manager 5. Because the statistical tests assessing heterogeneity generally have a lack of power because of the low number of studies combined, significance was set at a $\mathrm{P}$ value of .10. If statistical tests showed heterogeneity, we used random-effect models. In the case of non-significant heterogeneity, we used fixed-effect models. We explored confounding using a sensitivity analysis. If there was ambiguity about whether a study met the inclusion criteria, we examined the effect of including or excluding this study. Where data pooling was not possible, statistically significant results are presented.

\section{RESULTS}

The literature search yielded 613 citations up to November 1, 2010. Removal of duplicates left 453 articles. Scrutinizing the titles and abstracts of these articles identified 58 potentially relevant studies, 11,12,16,17,48,49,56-59,62-109 which were retrieved for further screening. Examination of the reference lists of these articles added 1 study, ${ }^{110}$ for a total of 59 potentially relevant studies. The identified articles were reviewed to determine whether they met the inclusion criteria. This led to the exclusion of 46 articles. Reason for exclusion included not reporting power or work $(n=26), 59,62-64,68,73,74,78,82-85,87-89,91,94-96,99,101,102,104,107-109$ only presenting power or work of prosthetic components $(n=6),{ }^{76,77,86,92,93,100}$, no numeric data or statistical analysis $(n=6)^{49,69,73,80,81,90}$ no description on individual joint level $(n=4), 17,70,98,105$ no comparisons between legs $(n=3),{ }^{11,12,67}$ and no optimal prosthetic prescription $(n=1){ }^{83}$ The remaining 13 articles, $16,48,56-58,65,66,72,79,97,103,106,110$ all of which used an observational design, were included in this systematic review. The flow diagram of article retrieval and analysis is displayed in Figure 2.1.

\section{Description of studies of TT amputation}

A total of 12 studies of TT amputation were identified. ${ }^{16,56-58,65,66,72,79,97,103,106,110}$. Five studies described work, and 9 studies described power. Details of included studies are presented in Table 2.1. Combining results of multiple trials studying a specific foot was not possible. We discussed whether the pooling of the different studies was legitimate based on the prosthetic components that were used in the trials, which was the case. 


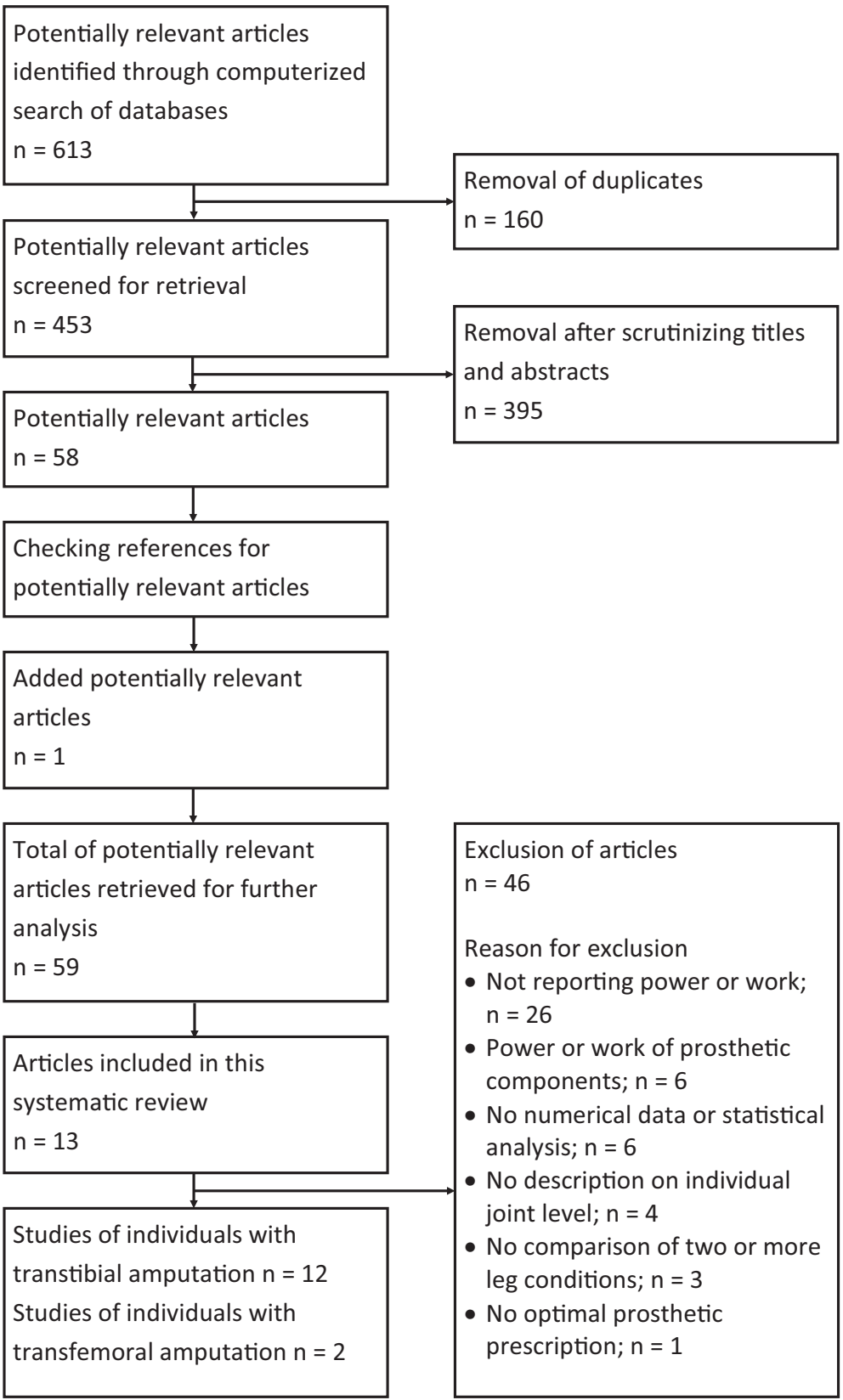

Figure 2.1: Flow diagram of article retrieval and analysis 
Table 2.1: Characteristics of studies of patients with a transtibial amputation

\begin{tabular}{|c|c|c|c|c|}
\hline Author (year) & \multicolumn{2}{|c|}{ Bateni and Olney (2002) } & \multicolumn{2}{|c|}{ Beyeart et al. (2008) } \\
\hline Design & \multicolumn{2}{|l|}{ Case series } & \multicolumn{2}{|l|}{ Case control } \\
\hline $\begin{array}{l}\text { Number of } \\
\text { participants }\end{array}$ & \multicolumn{2}{|l|}{$\begin{array}{l}\text { Amputees: } 5 \\
\text { Controls: None }\end{array}$} & \multicolumn{2}{|l|}{$\begin{array}{l}\text { Amputees: } 17 \\
\text { Controls: } 15\end{array}$} \\
\hline Age (years) & \multicolumn{2}{|c|}{ Amputees: $50.6 \pm 14.5$} & \multicolumn{2}{|c|}{$\begin{array}{l}\text { Amputees: } 46 \pm 16 \\
\text { Controls: } 45 \pm 17\end{array}$} \\
\hline $\begin{array}{l}\text { Inclusion } \\
\text { criteria }\end{array}$ & \multicolumn{2}{|c|}{$\begin{array}{l}\text { Amputees: using Seattle Light foot } \\
\text { prosthesis; fully ambulatory; normally } \\
\text { performing prosthesis } \\
\text { Controls: Matched for age, height, } \\
\text { mass and sex; no known } \\
\text { musculoskeletal problems affecting } \\
\text { gait }\end{array}$} & \multicolumn{2}{|c|}{$\begin{array}{l}\text { Amputees: Unilateral amputation; } \\
\text { prosthesis use > } 1 \text { year; not walking } \\
\text { with assistive devices; no stump pain, } \\
\text { tenderness; no cardiovascular, } \\
\text { neurologic, or musculoskeletal } \\
\text { abnormalities affecting gait } \\
\text { Controls: Age- and height-matched } \\
\text { healthy subjects }\end{array}$} \\
\hline $\begin{array}{l}\text { Reason for } \\
\text { amputation }\end{array}$ & \multicolumn{2}{|l|}{$\begin{array}{l}\text { Congenital: } 4 \\
\text { Infection: } 2\end{array}$} & \multicolumn{2}{|l|}{ Traumatic } \\
\hline $\begin{array}{l}\text { Time since } \\
\text { amputation }\end{array}$ & \multicolumn{2}{|l|}{ Not available } & \multicolumn{2}{|l|}{$16.7 \pm 17.6$ years } \\
\hline Used foot & \multicolumn{2}{|l|}{ Seattle Light Foot } & \multicolumn{2}{|c|}{ Propulsive feet (15) and SACH (2) } \\
\hline $\begin{array}{l}\text { Outcome } \\
\text { measures }\end{array}$ & \multicolumn{2}{|c|}{$\begin{array}{l}\text { Peak Power K1/K2/K3/K4 (W/Kg) } \\
\text { Amputated, intact, and referent leg }\end{array}$} & \multicolumn{2}{|c|}{$\begin{array}{l}\text { Work K1/K2 (J/Kg) } \\
\text { Amputated leg, intact leg, and referent } \\
\text { leg }\end{array}$} \\
\hline $\begin{array}{l}\text { Walking speed } \\
(\mathrm{m} / \mathrm{s})\end{array}$ & \multicolumn{2}{|c|}{$\begin{array}{l}\text { Amputees: } 1.04 \pm 0.1 \\
\text { Controls: } 1.12 \pm 0.17\end{array}$} & \multicolumn{2}{|c|}{$\begin{array}{l}\text { Amputees: } 1.36 \pm 0.20 \\
\text { Controls: } 1.39 \pm 0.17\end{array}$} \\
\hline $\begin{array}{l}\text { Methodologic } \\
\text { quality }\end{array}$ & $\begin{array}{l}\text { Reporting } \\
\text { External validity } \\
\text { Internal validity } \\
\text { Selection bias } \\
\text { Power } \\
\text { Total }\end{array}$ & $\begin{array}{l}6 / 11 \\
0 / 3 \\
4 / 7 \\
1 / 6 \\
0 / 5 \\
12\end{array}$ & $\begin{array}{l}\text { Reporting } \\
\text { External validity } \\
\text { Internal validity } \\
\text { Selection bias } \\
\text { Power } \\
\text { Total }\end{array}$ & $\begin{array}{l}8 / 11 \\
1 / 3 \\
5 / 7 \\
1 / 6 \\
0 / 5 \\
15\end{array}$ \\
\hline
\end{tabular}

Data are presented as mean \pm standard deviation unless otherwise stated

Table continues on next page 
Table 2.1: Characteristics of studies of patients with a transtibial amputation (continued)

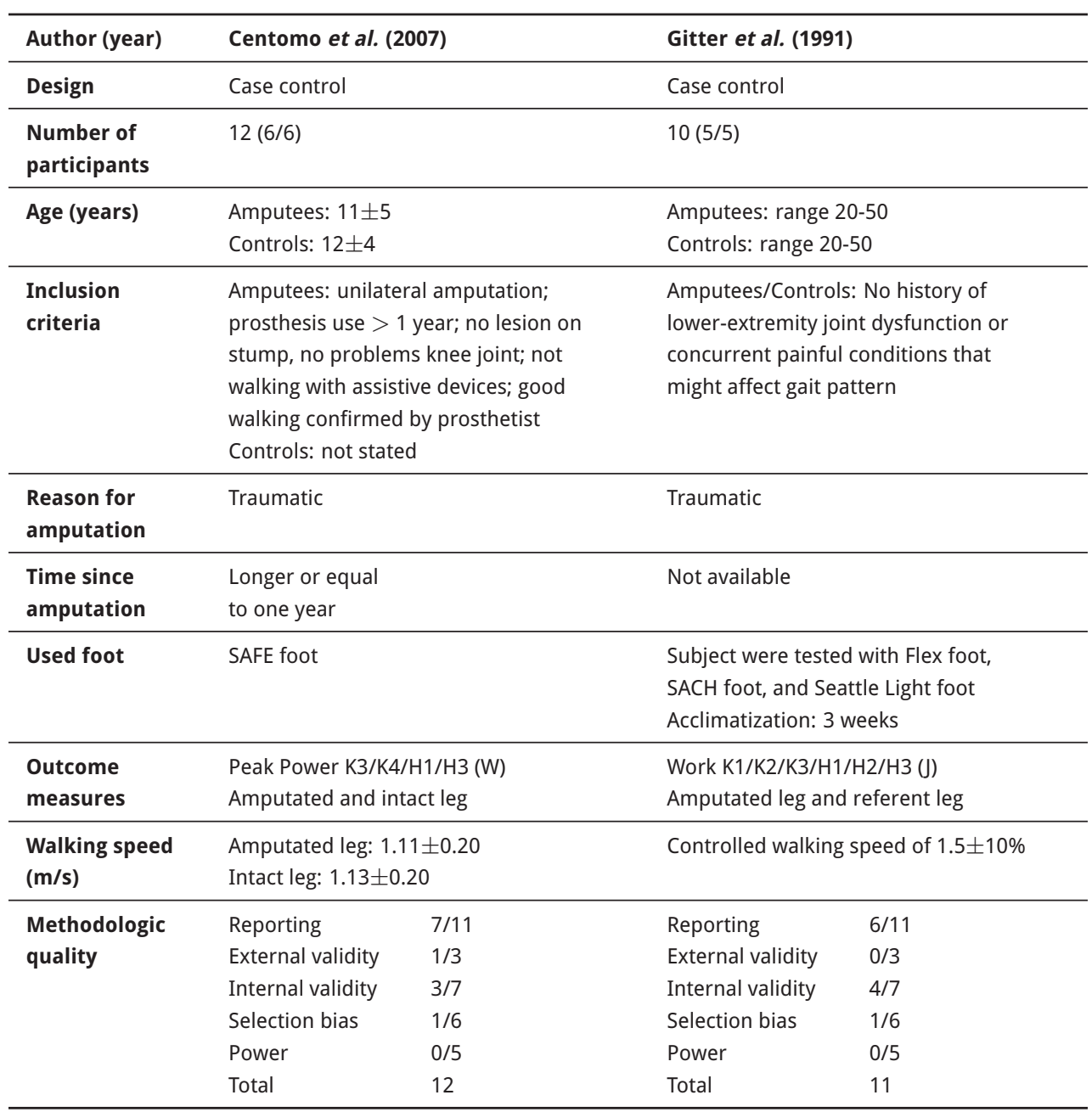

Data are presented as mean \pm standard deviation unless otherwise stated

Table continues on next page 
Table 2.1: Characteristics of studies of patients with a transtibial amputation (continued)

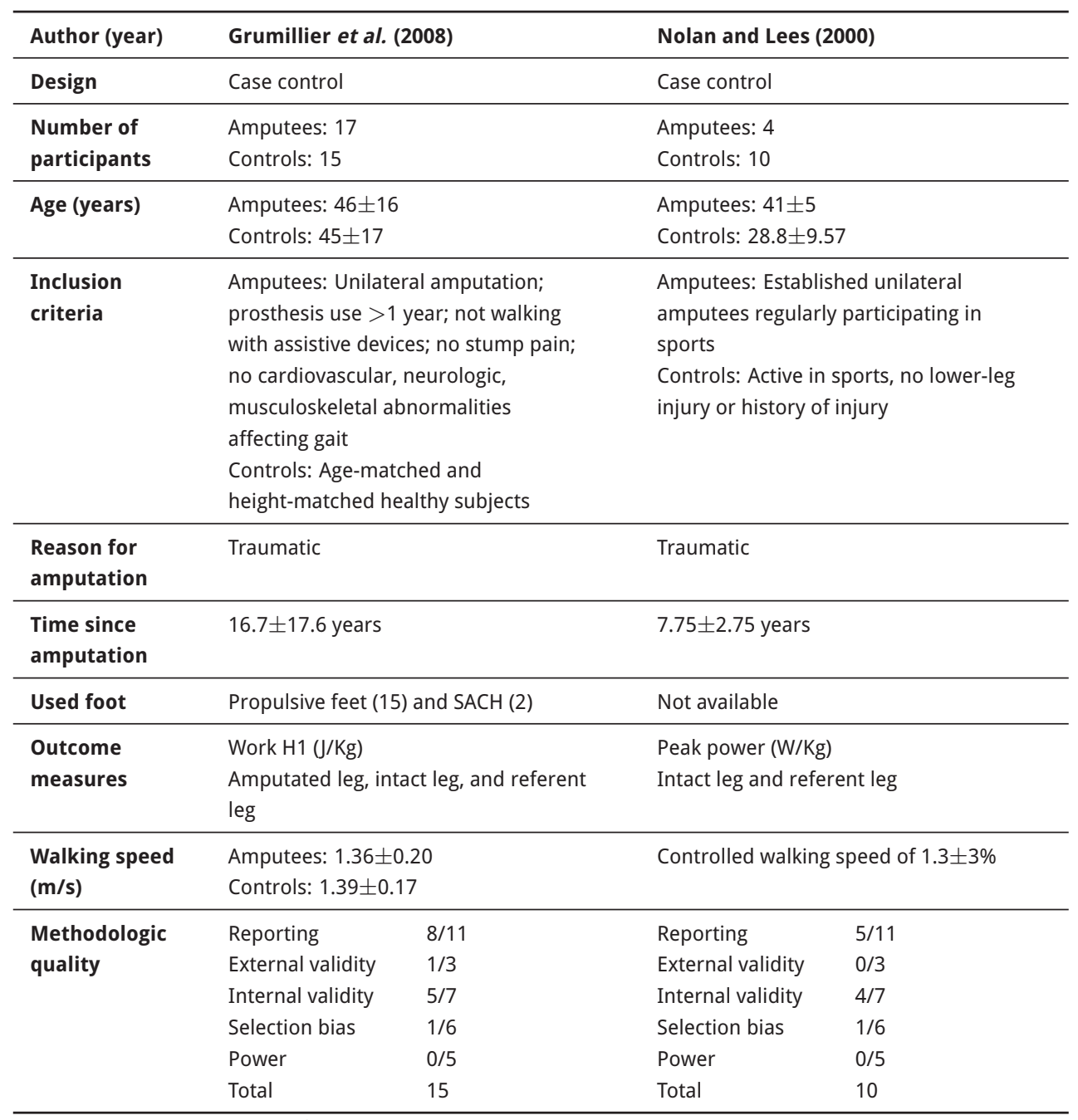

Data are presented as mean \pm SD unless otherwise stated

Table continues on next page 
Table 2.1: Characteristics of studies of patients with a transtibial amputation (continued)

\begin{tabular}{|c|c|c|c|c|}
\hline Author (year) & \multicolumn{2}{|c|}{ Powers et al. (1998) } & \multicolumn{2}{|c|}{ Sadeghi et al. (2001) } \\
\hline Design & \multicolumn{2}{|l|}{ Case control } & \multicolumn{2}{|l|}{ Case series } \\
\hline $\begin{array}{l}\text { Number of } \\
\text { participants }\end{array}$ & \multicolumn{2}{|l|}{$\begin{array}{l}\text { Amputees: } 10 \\
\text { Controls: } 10\end{array}$} & \multicolumn{2}{|l|}{$\begin{array}{l}\text { Amputees: } 5 \\
\text { No controls }\end{array}$} \\
\hline Age (years) & \multicolumn{2}{|c|}{$\begin{array}{l}\text { Amputees: } 62.3 \pm 6.9 \\
\text { Controls: } 50.9 \pm 8.6\end{array}$} & \multicolumn{2}{|l|}{$27 \pm 12.7$} \\
\hline $\begin{array}{l}\text { Inclusion } \\
\text { criteria }\end{array}$ & \multicolumn{2}{|c|}{$\begin{array}{l}\text { Amputees: Unilateral vascular } \\
\text { amputation; independent community } \\
\text { ambulators; no use of assistive devices } \\
\text { Controls: Free of any conditions } \\
\text { affecting gait }\end{array}$} & \multicolumn{2}{|c|}{$\begin{array}{l}\text { Not explicitly stated; all subjects were } \\
\text { unilateral amputees without stump } \\
\text { problems }\end{array}$} \\
\hline $\begin{array}{l}\text { Reason for } \\
\text { amputation }\end{array}$ & \multicolumn{2}{|l|}{ Vascular } & \multicolumn{2}{|l|}{$\begin{array}{l}\text { Traumatic: } 3 \\
\text { Vascular: } 2\end{array}$} \\
\hline $\begin{array}{l}\text { Time since } \\
\text { amputation }\end{array}$ & \multicolumn{2}{|l|}{$>2$ years } & \multicolumn{2}{|l|}{ Not available } \\
\hline Used foot & \multicolumn{2}{|l|}{ Seattle Light foot } & \multicolumn{2}{|l|}{$\mathrm{SACH}$} \\
\hline $\begin{array}{l}\text { Outcome } \\
\text { measures }\end{array}$ & \multicolumn{2}{|c|}{$\begin{array}{l}\text { Peak positive knee power during } \\
\text { stance (W/Kg-m). } \\
\text { Amputated leg and referent leg }\end{array}$} & \multicolumn{2}{|c|}{$\begin{array}{l}\text { Peak power } \mathrm{K} 1 / \mathrm{K} 2 / \mathrm{K} 3 / \mathrm{K} 4 / \mathrm{H} 1 / \mathrm{H} 2 / \mathrm{H} 3 \\
(\mathrm{~W} / \mathrm{Kg}) \\
\text { Amputated and intact leg }\end{array}$} \\
\hline $\begin{array}{l}\text { Walking speed } \\
(\mathrm{m} / \mathrm{s})\end{array}$ & \multicolumn{2}{|c|}{$\begin{array}{l}\text { Amputees: } 1.08 \pm 0.18 \\
\text { Controls: } 1.30 \pm 0.20\end{array}$} & \multicolumn{2}{|c|}{$\begin{array}{l}\text { Amputated leg: } 1.27 \pm 0.22 \\
\text { Intact leg: } 1.28 \pm 0.22\end{array}$} \\
\hline $\begin{array}{l}\text { Methodologic } \\
\text { quality }\end{array}$ & $\begin{array}{l}\text { Reporting } \\
\text { External validity } \\
\text { Internal validity } \\
\text { Selection bias } \\
\text { Power } \\
\text { Total }\end{array}$ & $\begin{array}{l}8 / 11 \\
1 / 3 \\
5 / 7 \\
1 / 6 \\
0 / 5 \\
15\end{array}$ & $\begin{array}{l}\text { Reporting } \\
\text { External validity } \\
\text { Internal validity } \\
\text { Selection bias } \\
\text { Power } \\
\text { Total }\end{array}$ & $\begin{array}{l}8 / 11 \\
1 / 3 \\
4 / 7 \\
2 / 6 \\
0 / 5 \\
15\end{array}$ \\
\hline
\end{tabular}

Data are presented as mean \pm standard deviation unless otherwise stated

Table continues on next page 
Table 2.1: Characteristics of studies of patients with a transtibial amputation (continued)

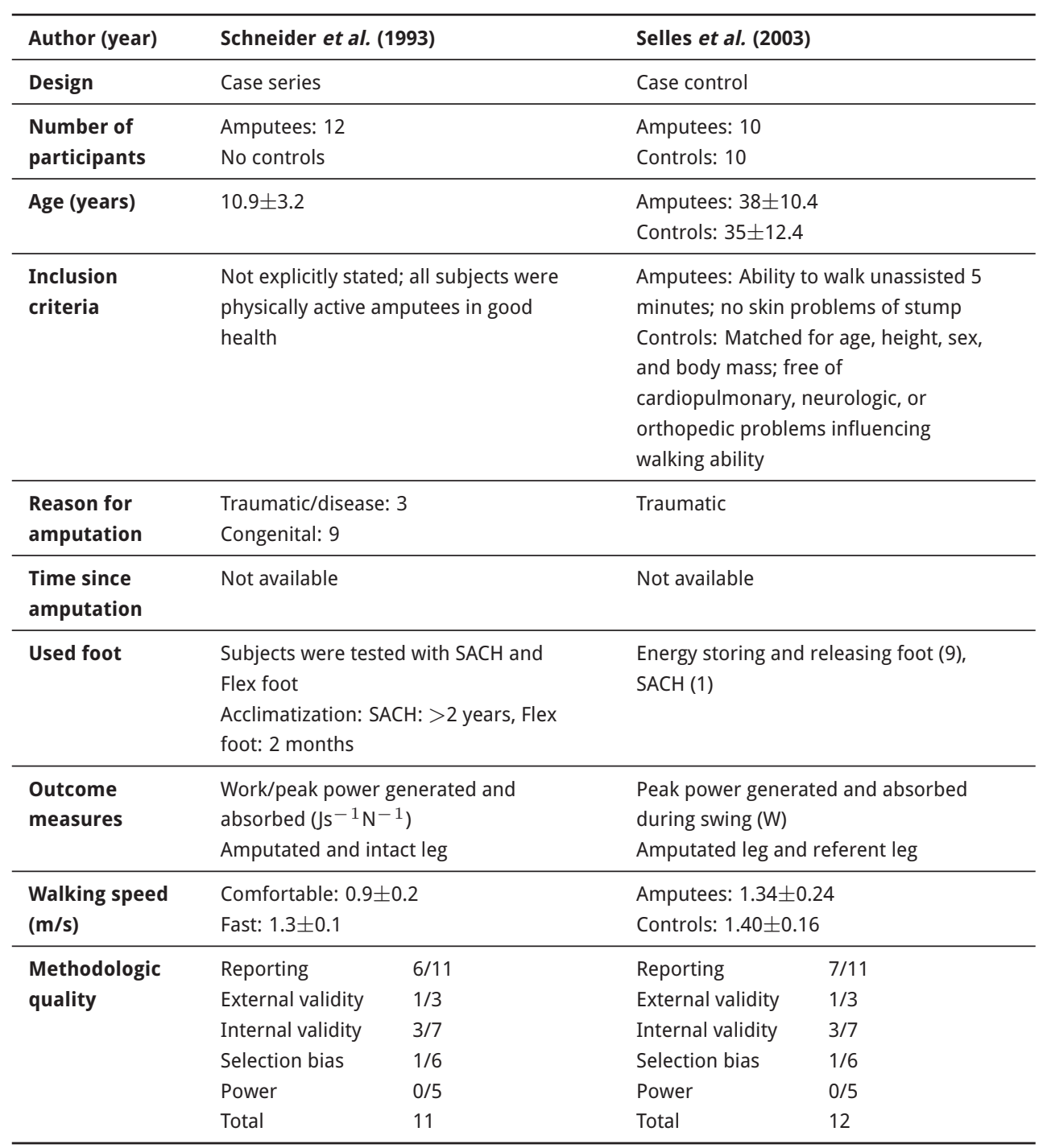

Data are presented as mean \pm standard deviation unless otherwise stated

Table continues on next page 
Table 2.1: Characteristics of studies of patients with a transtibial amputation (continued)

\begin{tabular}{|c|c|c|c|c|}
\hline Author (year) & \multicolumn{2}{|c|}{ Silverman et al. (2008) } & \multicolumn{2}{|c|}{ Vanicek et al. (2009) } \\
\hline Design & \multicolumn{2}{|l|}{ Case control } & \multicolumn{2}{|l|}{ Case control } \\
\hline $\begin{array}{l}\text { Number of } \\
\text { participants }\end{array}$ & \multicolumn{2}{|l|}{$\begin{array}{l}\text { Amputees: } 14 \\
\text { Controls: } 10\end{array}$} & \multicolumn{2}{|l|}{$\begin{array}{l}\text { Fallers: } 6 \\
\text { Non-fallers: } 5\end{array}$} \\
\hline Age (years) & \multicolumn{2}{|l|}{$\begin{array}{l}\text { Amputees: } 45 \pm 9 \\
\text { Controls: } 33 \pm 12\end{array}$} & \multicolumn{2}{|c|}{$\begin{array}{l}\text { Fallers: } 56 \pm 13 \\
\text { Non-fallers: } 57 \pm 21\end{array}$} \\
\hline $\begin{array}{l}\text { Inclusion } \\
\text { criteria }\end{array}$ & \multicolumn{2}{|c|}{$\begin{array}{l}\text { Both groups: Free from } \\
\text { musculoskeletal disorders and leg } \\
\text { pain; not requiring assistive devices; } \\
\text { proficient walkers }\end{array}$} & \multicolumn{2}{|c|}{$\begin{array}{l}\text { Both groups: Wearing prosthesis on } \\
\text { daily basis; ability to walk } 120 \text { meters } \\
\text { without walking aids and experiencing } \\
\text { pain. } \\
\text { For the group of fallers, an additional } \\
\text { inclusion criterion was a fall within the } \\
\text { last } 9 \text { months. }\end{array}$} \\
\hline $\begin{array}{l}\text { Reason for } \\
\text { amputation }\end{array}$ & \multicolumn{2}{|l|}{$\begin{array}{l}\text { Traumatic: } 11 \\
\text { Vascular: } 3\end{array}$} & \multicolumn{2}{|c|}{$\begin{array}{l}\text { Fallers: Traumatic: 4, Vascular: } 2 \\
\text { Non-fallers: Traumatic: 3, Vascular: } 2\end{array}$} \\
\hline $\begin{array}{l}\text { Time since } \\
\text { amputation }\end{array}$ & \multicolumn{2}{|l|}{ Not available } & \multicolumn{2}{|c|}{$\begin{array}{l}\text { Fallers: } 3.5 \pm 4.3 \\
\text { Non-fallers: } 10.6 \pm 12.3\end{array}$} \\
\hline Used foot & \multicolumn{2}{|c|}{$\begin{array}{l}\text { Energy storing and releasing foot (9), } \\
\text { SACH (5) }\end{array}$} & \multicolumn{2}{|c|}{$\begin{array}{l}\text { Fallers: Multiflex (4), Variflex (1), } \\
\text { Ceterus (1) } \\
\text { Non-fallers: Multifex (3), Variflex (1), } \\
\text { Dynamic (1) }\end{array}$} \\
\hline $\begin{array}{l}\text { Outcome } \\
\text { measures }\end{array}$ & \multicolumn{2}{|c|}{$\begin{array}{l}\text { Concentric/eccentric work during } \\
\text { stance and peak power } \\
\mathrm{K} 1 / \mathrm{K} 2 / \mathrm{K} 3 / \mathrm{K} 4 / \mathrm{H} 1 / \mathrm{H} 2 / \mathrm{H} 3(\mathrm{~W} / \mathrm{Kg}) \\
\text { Amputated leg, intact leg and referent } \\
\text { leg }\end{array}$} & \multicolumn{2}{|c|}{$\begin{array}{l}\text { Peak power K1/K2/K3/K4/H1/H2/H3 } \\
\text { (W/Kg) } \\
\text { Amputated leg and intact leg }\end{array}$} \\
\hline $\begin{array}{l}\text { Walking speed } \\
(\mathrm{m} / \mathrm{s})\end{array}$ & \multicolumn{2}{|c|}{$\begin{array}{l}\text { Controlled walking speed of } 0.6,0.9, \\
1.2 \text { and } 1.5\end{array}$} & \multicolumn{2}{|c|}{$\begin{array}{l}\text { Fallers: } 1.19 \pm 0.35 \\
\text { Non-fallers: } 1.07 \pm 0.20\end{array}$} \\
\hline $\begin{array}{l}\text { Methodologic } \\
\text { quality }\end{array}$ & $\begin{array}{l}\text { Reporting } \\
\text { External validity } \\
\text { Internal validity } \\
\text { Selection bias } \\
\text { Power } \\
\text { Total }\end{array}$ & $\begin{array}{l}8 / 11 \\
0 / 3 \\
4 / 7 \\
2 / 6 \\
0 / 5 \\
14\end{array}$ & $\begin{array}{l}\text { Reporting } \\
\text { External validity } \\
\text { Internal validity } \\
\text { Selection bias } \\
\text { Power } \\
\text { Total }\end{array}$ & $\begin{array}{l}8 / 11 \\
1 / 3 \\
4 / 7 \\
2 / 6 \\
0 / 5 \\
15\end{array}$ \\
\hline
\end{tabular}

Data are presented as mean \pm standard deviation unless otherwise stated 


\section{Joint work, amputated-intact legs}

Four studies compared joint work of amputated and intact legs. ${ }^{57,66,103,110}$ For the trial by Silverman et al., ${ }^{57}$ only the data obtained at a walking speed of $1.2 \mathrm{~m} / \mathrm{s}$ were used in the analysis. Methodologic quality ranged from $12^{103}$ to $15 .{ }^{66,110}$ Three studies ${ }^{66,103,110}$ presented results according to the categorization of Winter. ${ }^{13}$ Of these trials, 1 trial presented results on the knee level ${ }^{66}$ and 2 trials on the hip level. ${ }^{103,110}$ The fourth study ${ }^{57}$ did not describe work according to the categorization of Winter ${ }^{13}$ but integrated the positive and negative power profile as a whole. The results of the 2 trials reporting on the hip level could be pooled. In addition to the pooled results, significant found in the other studies results are presented.

\section{Joint work, amputated-referent legs}

Four studies compared joint work of the amputated leg and a referent leg. ${ }^{56,57,66,110}$ Methodologic quality ranged from $11^{56}$ to $15 .{ }^{66,110}$ Three studies reported on the knee level, ${ }^{56,57,66}$ and 3 studies reported on the hip level. $57,103,110$ These studies could not be pooled because they made use of different measurements units. Significant results are presented.

\section{Joint work, intact-referent legs}

Three studies compared joint work of the intact leg and a referent leg. ${ }^{57,66,110}$ Methodologic quality ranged from $14^{57}$ to $15 .{ }^{57,66}$ Two studies ${ }^{66,110}$ used the categorization of Winter ${ }^{13}$ and presented results on the knee ${ }^{66}$ and hip ${ }^{110}$ levels. The third study ${ }^{57}$ did not use the categorization of Winter. ${ }^{13}$ None of the studies could be pooled. Significant results are presented.

\section{Joint power, amputated-intact legs}

Six studies compared joint power of the amputated and intact legs. ${ }^{57,58,65,79,103,106}$ In the study of Silverman et al. ${ }^{57}$ and the trial of Vanicek et al. ${ }^{65}$ results of power generation and absorption are graphically presented. Therefore, the primary authors were contacted to obtain numeric data. These data were provided. The trial of Vanicek et al. ${ }^{65}$ made a comparison between fallers and non-fallers. Because falling was not an exclusion criterion for the present review, we chose to include data of both groups. Methodologic quality ranged from $11^{79}$ to 15 points. ${ }^{58,65}$ Of the 6 studies, 4 studies could be pooled. ${ }^{57,58,65,103}$ The other studies could not be pooled because 1 study did not present a graphic or numeric representation ${ }^{106}$ and 1 study described the overall peak values of power generation and absorption ${ }^{79}$ instead of peaks of the categories identified by Winter. ${ }^{13}$ Significant results of these 2 studies are presented next to the results of data pooling.

\section{Joint power, amputated-referent legs}

Four studies compared the joint power of the amputated leg and a referent leg. $57,72,97,106$ Methodologic quality ranged from $12^{72}$ to $15 .{ }^{97}$ Only the results of 2 studies regarding the K4 phase could be pooled. ${ }^{57,72}$ The other studies could not be pooled because the study 
of Centomo et al. ${ }^{106}$ failed to report numeric data, and the trial of Powers et al. ${ }^{97}$ failed to report a standard deviation. Significant results found in the individual studies are presented next to the pooled data.

\section{Joint power, intact-referent legs}

Three studies compared joint power of the intact leg and a referent leg. ${ }^{16,57,106}$ Methodologic quality ranged from $10^{16}$ to $14^{57}$ points. The data of 2 trials could be pooled. ${ }^{57,106}$ Both trials reported on the $\mathrm{A} 1, \mathrm{~A} 2, \mathrm{~K} 2, \mathrm{~K} 3, \mathrm{H} 2$, and $\mathrm{H} 3$ phases. Silverman et al. ${ }^{57}$ additionally presented data on the other phases. The data of the trial of Centomo et al. ${ }^{106}$ could not be pooled because no numeric data were presented. Significant results are presented.

\section{Results of studies of TT amputation}

Results of trials are displayed in Table 2.2 and Table 2.3.

\section{Joint work, amputated-intact legs}

Results showed that the knee of the intact leg performed more concentric work than the knee of the amputated leg. ${ }^{57}$ Looking more specifically at the different phases, significantly less eccentric work was performed during the $\mathrm{K} 1$ phase of the amputated leg compared with the intact leg. ${ }^{66}$ During the K2 phase, significantly less concentric work was performed by the amputated leg than by the intact leg. ${ }^{66}$ On the hip level, no significant differences were found. $57,103,110$

\section{Joint work, amputated-referent legs}

Results indicated that the knee of the amputated leg performed significantly less concentric work during the stance phase than the knee of a referent leg. ${ }^{57}$ The hip of the amputated leg performed significantly more concentric work than the hip of a referent leg during the stance phase. ${ }^{57}$ Looking more specifically at the phases of the knee, conflicting results were found. The trial of Gitter et al. ${ }^{56}$ found that in the K2 phase, significantly less work was performed by the amputated leg when wearing the Flex foot, whereas the study of Beyaert et al. ${ }^{66}$ found no significant differences. When looking at the hip, results showed that during the $\mathrm{H} 1$ phase, significantly more work was performed by the amputated leg than a referent leg. 56,110

\section{Joint work, intact-referent legs}

Results indicated that the hip and knee of the intact leg performed significantly more concentric work during the stance phase than the hip and knee of a referent leg. ${ }^{57}$ When looking more specifically at the different phases of the knee, results indicated that during the K1 phase, ${ }^{66}$ the $\mathrm{K} 2$ phase, ${ }^{66}$ and the $\mathrm{H} 1$ phase, ${ }^{110}$ significantly more work was performed by the intact leg than a referent leg. 
Table 2.2: Results of Joint Work of patients with a transtibial amputation

\begin{tabular}{|c|c|c|c|c|c|c|c|c|}
\hline & & & & \multicolumn{5}{|c|}{ Intact Leg } \\
\hline & \multicolumn{3}{|c|}{ Parameters } & \multicolumn{2}{|c|}{ Result Comparison } & \multicolumn{3}{|c|}{ Mean \pm SD } \\
\hline \multirow{7}{*}{ 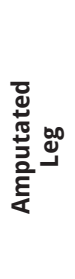 } & Knee & K1 & Work & $\mathrm{AL}<\mathrm{IL}$ & $P<.05$ & $-2.8 \pm 1.8$ & vs & $-10 \pm 5$ \\
\hline & & $\mathrm{K} 2$ & Work & $\mathrm{AL}<\mathrm{IL}$ & $P<.05$ & $2.4 \pm 2.1$ & vs & $4.6 \pm 3.2$ \\
\hline & & Conc & Work Stance & $\mathrm{AL}<\mathrm{IL}$ & $P<.05$ & & - & \\
\hline & & Ecc & Work Stance & $\mathrm{AL}<\mathrm{IL}$ & NS & & - & \\
\hline & Hip & $\mathrm{H} 1$ & Work & $\mathrm{AL}>\mathrm{IL}$ & NS & 0.01 & $(-0$. & $, 0.05)$ \\
\hline & & Conc & Work Stance & $\mathrm{AL}<\mathrm{IL}$ & NS & & - & \\
\hline & & Ecc & Work Stance & $\mathrm{AL}<\mathrm{IL}$ & NS & & - & \\
\hline
\end{tabular}

\begin{tabular}{|c|c|c|c|c|c|c|c|c|}
\hline & & & & \multicolumn{5}{|c|}{ Referent Leg } \\
\hline & \multicolumn{3}{|c|}{ Parameters } & \multicolumn{2}{|c|}{ Result Comparison } & \multicolumn{3}{|c|}{ Mean \pm SD } \\
\hline \multirow{7}{*}{ 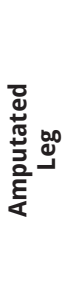 } & Knee & K1 & Work & $\mathrm{AL}<\mathrm{RL}$ & NS & $-2.8 \pm 1.8$ & vs & $-3.5 \pm 2.4$ \\
\hline & & K2 & Work & \multicolumn{5}{|c|}{ Conflicting evidence } \\
\hline & & Conc & Work Stance & $\mathrm{AL}<\mathrm{RL}$ & $P<.05$ & & - & \\
\hline & & Ecc & Work Stance & $\mathrm{AL}<\mathrm{RL}$ & NS & & - & \\
\hline & Hip & $\mathrm{H} 1$ & Work & $\mathrm{AL}>\mathrm{RL}$ & $P<.05$ & $17.1 \pm 6.0$ & vs & $14.2 \pm 4.9$ \\
\hline & & Conc & Work Stance & $\mathrm{AL}>\mathrm{RL}$ & $P<.05$ & & - & \\
\hline & & Ecc & Work Stance & $\mathrm{AL}<\mathrm{RL}$ & NS & & - & \\
\hline
\end{tabular}

\begin{tabular}{|c|c|c|c|c|c|c|c|c|}
\hline & & & & \multicolumn{5}{|c|}{ Intact Leg } \\
\hline & \multicolumn{3}{|c|}{ Parameters } & \multicolumn{2}{|c|}{ Result Comparison } & \multicolumn{3}{|c|}{ Mean \pm SD } \\
\hline \multirow{9}{*}{ 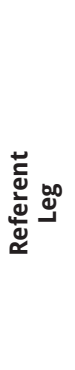 } & \multirow[t]{2}{*}{ Ankle } & \multirow{2}{*}{$\begin{array}{l}\text { Conc } \\
\text { Ecc }\end{array}$} & \multirow{2}{*}{$\begin{array}{l}\text { Work Stance } \\
\text { Work Stance }\end{array}$} & \multirow{2}{*}{$\begin{array}{l}\mathrm{RL}<\mathrm{IL} \\
\mathrm{RL}<\mathrm{IL}\end{array}$} & \multirow{2}{*}{$\begin{array}{l}\text { NS } \\
\text { NS }\end{array}$} & \multicolumn{3}{|c|}{-} \\
\hline & & & & & & \multicolumn{3}{|c|}{-} \\
\hline & \multirow[t]{4}{*}{ Knee } & K1 & Work & $\mathrm{RL}<\mathrm{IL}$ & $P<.05$ & $-3.5 \pm 2.4$ & vs & $-10 \pm 5$ \\
\hline & & $\mathrm{K} 2$ & Work & $\mathrm{RL}<\mathrm{IL}$ & $P<.05$ & $2.6 \pm 1.7$ & vs & $4.6 \pm 3.2$ \\
\hline & & Conc & Work Stance & $\mathrm{RL}<\mathrm{IL}$ & NS & & - & \\
\hline & & Ecc & Work Stance & $\mathrm{RL}>\mathrm{IL}$ & NS & & - & \\
\hline & \multirow[t]{3}{*}{ Hip } & $\mathrm{H} 1$ & Work & $\mathrm{RL}<\mathrm{IL}$ & $P<.05$ & $14.2 \pm 4.9$ & vs & $16.9 \pm 8.4$ \\
\hline & & Conc & Work Stance & $\mathrm{RL}<\mathrm{IL}$ & $P<.05$ & & - & \\
\hline & & Ecc & Work Stance & $\mathrm{RL}>\mathrm{IL}$ & NS & & - & \\
\hline
\end{tabular}

Abbreviations: NS, non-significant; Conc, concentric; Ecc, eccentric; AL, Amputated

\section{Joint power, amputated-intact legs}

Data pooling showed that during the K4 phase, significantly less power was absorbed in the amputated leg than the intact leg. ${ }^{57,58,65}$ During the $\mathrm{H} 2$ phase, significantly more power was absorbed in the amputated leg. ${ }^{57,58,65}$ In contrast with the first finding, the study by Centomo et al. ${ }^{106}$ found no statistically significant differences during all knee phases. Schneider et al. ${ }^{79}$ found a significant decrease in peak power absorption of the knee of the amputated leg when wearing the SACH and Flex foot compared with the intact leg. The peak power 
generation of the knee was significantly lower in the amputated leg when wearing the Flex foot. On the hip level, the peak power absorption was significantly decreased and the peak power generation was increased in the amputated leg in both feet conditions compared with the intact leg.

\section{Joint power, amputated-referent legs}

During the stance phase, significantly less knee power was generated in the amputated leg than in a referent leg. ${ }^{57,97}$ When looking at the swing phase, peak power generation of the knee was significantly lower in the amputated leg than in a referent leg. ${ }^{72}$ In contrast with these findings, Centomo et al. ${ }^{106}$ found no significant differences on the knee level.

\section{Joint power, intact-referent legs}

No significant differences were found.

\section{Description of studies of TF amputation}

Two studies of TF amputation were identified. ${ }^{16,48}$ One study described performed work, ${ }^{48}$ and 1 study described power. ${ }^{16}$ Details of included studies are described in Table 2.4.

In the study describing performed work, ${ }^{48}$ reasons for amputation included trauma and a tumor. Amputees used a Mauch SNS in combination with a Seattle Light foot. The methodologic quality of this study was 13 points.

One study presented joint power of the intact leg compared with a referent leg. ${ }^{16}$ All amputees participating in this study had trauma. The methodologic quality of this study was 10 points.

\section{Results of studies of TF amputation}

Results are displayed in Table 2.5.

\section{Joint work, amputated-intact legs}

Results showed that during the $\mathrm{H} 2$ phase, significantly more work was performed by the amputated leg than the intact leg. ${ }^{48}$

\section{Joint work, amputated-referent legs}

Results showed that in the $\mathrm{H} 2$ phase, significantly more work was performed by the amputated leg than the hip of a referent leg. ${ }^{48}$

\section{Joint work, intact-referent legs}

Results indicated that significantly more work was performed by the intact leg during the A2 phase than by a referent leg. During the $\mathrm{H} 1$ phase, significantly more work was performed by the intact leg. When looking at the $\mathrm{H} 2$ phase, significantly less work was performed by the intact leg than a referent leg. ${ }^{48}$ 
Table 2.3: Results of Joint Power of patients with a transtibial amputation

\begin{tabular}{|c|c|c|c|c|c|c|c|}
\hline & & & & \multicolumn{4}{|c|}{ Intact Leg } \\
\hline & \multicolumn{3}{|c|}{ Parameters } & \multicolumn{2}{|c|}{ Result Comparison } & \multicolumn{2}{|c|}{ Mean Diff $(95 \% \mathrm{CI})$} \\
\hline \multirow{7}{*}{ 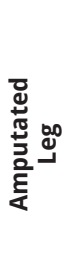 } & Knee & K1 & Power & $\mathrm{AL}<\mathrm{IL}$ & $P=.05$ & 0.35 & $(0.00,0.69)$ \\
\hline & & K2 & Power & $\mathrm{AL}>\mathrm{IL}$ & $P=.65$ & 0.03 & $(-0.07,0.22)$ \\
\hline & & K3 & Power & $\mathrm{AL}>\mathrm{IL}$ & $P=.12$ & -0.17 & $(-0.37,0.04)$ \\
\hline & & K4 & Power & $\mathrm{AL}<\mathrm{IL}$ & $P=.003$ & 0.27 & $(0.08,0.45)$ \\
\hline & Hip & $\mathrm{H} 1$ & Power & $\mathrm{AL}>\mathrm{IL}$ & $P=.73$ & 0.03 & $(-0.13,0.18)$ \\
\hline & & $\mathrm{H} 2$ & Power & $\mathrm{AL}>\mathrm{IL}$ & $P=.02$ & -0.15 & $(-0.27,-0.02)$ \\
\hline & & $\mathrm{H} 3$ & Power & $\mathrm{AL}<\mathrm{IL}$ & $P=.99$ & 0.00 & $(-0.15,0.15)$ \\
\hline
\end{tabular}

\begin{tabular}{|c|c|c|c|c|c|c|c|c|}
\hline & & & & & & ferent Leg & & \\
\hline & Parar & ters & & Result & mparison & Mean Diff & $5 \%$ & \\
\hline & Knee & K4 & Power & $\mathrm{AL}<\mathrm{RL}$ & $P=.31$ & 0.30 & & $(-0.29,0.89)$ \\
\hline & & Peak & Power Stance & $\mathrm{AL}<\mathrm{RL}$ & $P<.05$ & 0.80 & vs & 0.60 \\
\hline$\stackrel{\pi}{5}$ & & Peak & Power Swing & $\mathrm{AL}<\mathrm{RL}$ & $P=.02$ & $13.8 \pm 8.4$ & vs & $28.8 \pm 10.7$ \\
\hline $\bar{\xi}$ & Hip & Peak & Power Stance & $\mathrm{AL}<\mathrm{RL}$ & $P=.14$ & $43.9 \pm 13.9$ & vs & $56.7 \pm 30.7$ \\
\hline & & Peak & Power Swing & $A L>R L$ & $P=.05$ & $-3.7 \pm 3.0$ & vs & $-1.7 \pm 1.9$ \\
\hline
\end{tabular}

\begin{tabular}{|c|c|c|c|c|c|c|c|}
\hline & & & & & & ntact Leg & \\
\hline & Paran & ters & & Result & mparison & Mean Diff & \\
\hline & Ankle & $\mathrm{A} 1$ & Power & $\mathrm{RL}>\mathrm{IL}$ & $P=.92$ & 0.03 & $(-0.60,0.66)$ \\
\hline & & $\mathrm{A} 2$ & Power & $\mathrm{RL}<\mathrm{IL}$ & $P=.80$ & -0.06 & $(-0.57,0.44)$ \\
\hline ฮ์ & Knee & K2 & Power & $\mathrm{RL}<\mathrm{IL}$ & $P=.23$ & 0.36 & $(-0.23,0.96)$ \\
\hline 幽 & & K3 & Power & $\mathrm{RL}<\mathrm{IL}$ & $P=.57$ & -0.47 & $(-2.10,1.16)$ \\
\hline & Hip & $\mathrm{H} 2$ & Power & $\mathrm{RL}<\mathrm{IL}$ & $P=.57$ & -0.21 & $(-0.92,0.50)$ \\
\hline & & H3 & Power & $\mathrm{RL}>\mathrm{IL}$ & $P=.33$ & 0.41 & $(-0.41,1.23)$ \\
\hline
\end{tabular}

Abbreviations: NS, non-significant; Conc, concentric; Ecc, eccentric

\section{Joint power, intact-referent legs}

Results indicated that significantly less power was generated during the $\mathrm{K} 2$ phase of the knee of a referent leg compared with the knee of the intact leg. ${ }^{16}$

\section{Results of sensitivity analysis}

In the comparison of peak power of the amputated and intact legs of TT amputation, the trial of Vanicek et al. ${ }^{65}$ was included. This study compared biomechanical variables of fallers and non-fallers. Because falling was not an exclusion criterion for this review, we chose to include data of both groups. However, a significant difference in joint power of the intact limb was found between fallers and non-fallers during the $\mathrm{H} 2$ phase. Because other trials did not report on falling, we chose to exclude the data of the fallers to test the robustness 
Table 2.4: Characteristics of studies of patients with a transfemoral amputation

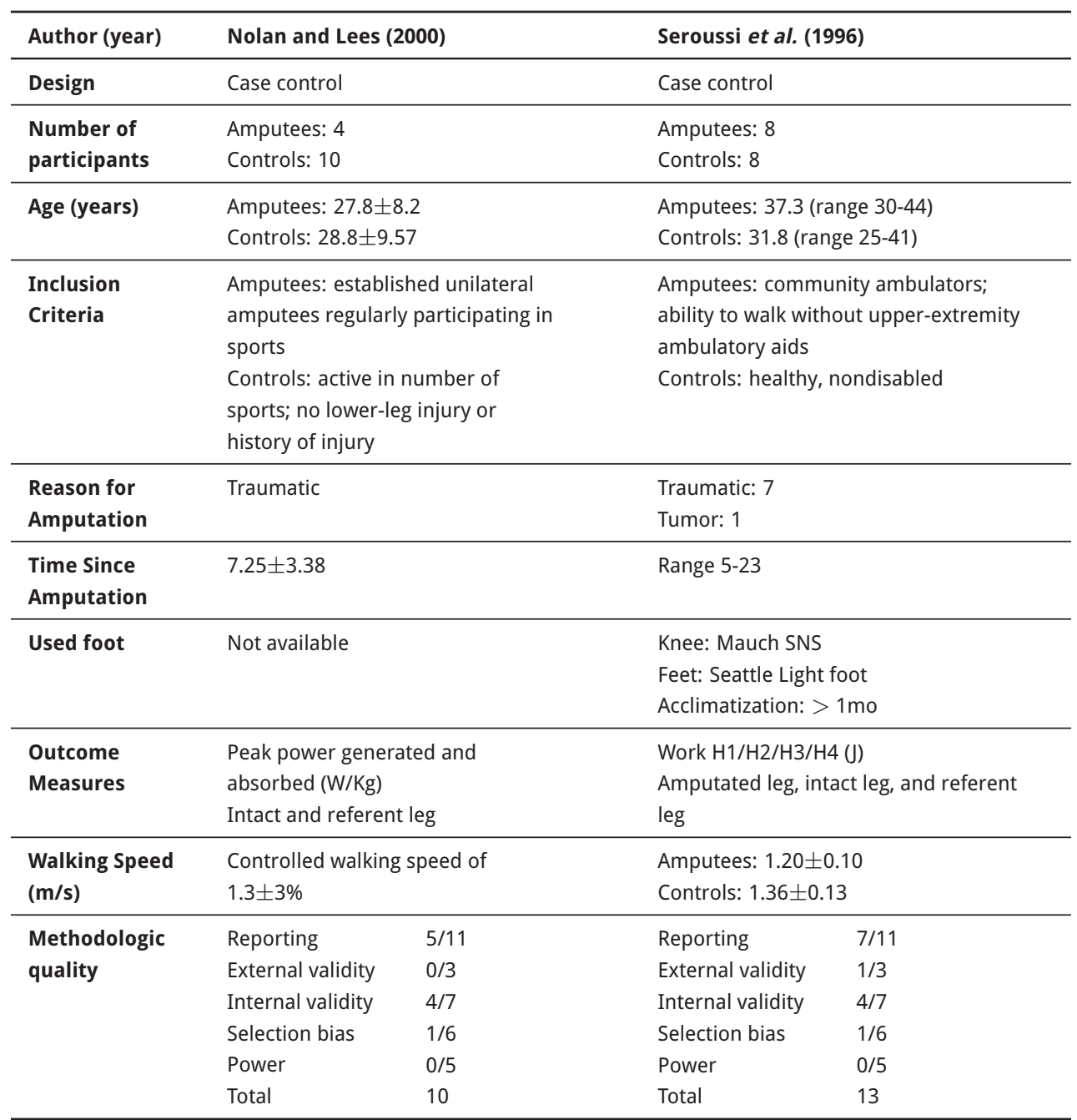

Data are presented as mean \pm standard deviation unless otherwise stated

of the found results. Exclusion of these data did not alter the described results.

\section{Discussion}

This systematic review was performed to describe the adaptation strategies of both the amputated leg and intact leg of TT and TF amputation. These adaptation strategies were described by comparing joint work and power of the amputated and intact legs and a referent leg. 
Table 2.5: Results of patients with a transfemoral amputation

\begin{tabular}{|c|c|c|c|c|c|c|c|c|}
\hline & & & & \multicolumn{5}{|c|}{ Intact Leg } \\
\hline \multicolumn{4}{|c|}{ Parameters } & \multicolumn{2}{|c|}{ Result Comparison } & \multicolumn{2}{|c|}{ Mean \pm SD } & \\
\hline \multirow{5}{*}{ 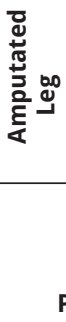 } & Hip & $\mathrm{H} 1$ & Work & $\mathrm{AL}<\mathrm{IL}$ & NS & $6.0 \pm 5.7$ & vs & $9.9 \pm 5.5$ \\
\hline & & $\mathrm{H} 2$ & Work & $\mathrm{AL}>\mathrm{IL}$ & $P<.02$ & $-13.6 \pm 2.5$ & vs & $-2.5 \pm 2.6$ \\
\hline & & $\mathrm{H} 3$ & Work & $\mathrm{AL}>\mathrm{IL}$ & NS & $8.7 \pm 3.5$ & vs & $7.3 \pm 2.8$ \\
\hline & & & & \multicolumn{5}{|c|}{ Referent Leg } \\
\hline & \multicolumn{2}{|c|}{ Parameters } & & \multicolumn{2}{|c|}{ Result Comparison } & \multicolumn{2}{|l|}{ Mean \pm SD } & \\
\hline \multirow{3}{*}{ 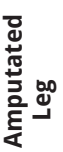 } & Hip & $\mathrm{H} 1$ & Work & $\mathrm{AL}>\mathrm{RL}$ & NS & $6.0 \pm 5.7$ & vs & $3.6 \pm 2.5$ \\
\hline & & $\mathrm{H} 2$ & Work & $\mathrm{AL}>\mathrm{RL}$ & $P<.02$ & $-13.5 \pm 5.6$ & vs & $-8.3 \pm 3.3$ \\
\hline & & $\mathrm{H} 3$ & Work & $\mathrm{AL}<\mathrm{RL}$ & NS & $8.7 \pm 3.5$ & vs & $9.0 \pm 1.9$ \\
\hline
\end{tabular}

\begin{tabular}{|c|c|c|c|c|c|c|c|c|}
\hline \multirow{2}{*}{\multicolumn{4}{|c|}{ Parameters }} & \multicolumn{5}{|c|}{ Intact Leg } \\
\hline & & & & \multicolumn{2}{|c|}{ Result Comparison } & \multicolumn{2}{|c|}{ Mean \pm SD } & \multirow[b]{2}{*}{$-10.3 \pm 3.3$} \\
\hline \multirow{15}{*}{ 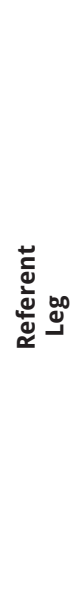 } & Ankle & $\mathrm{A} 1$ & Work & $\mathrm{RL}<\mathrm{IL}$ & NS & $-8.6 \pm 3.6$ & vs & \\
\hline & & $\mathrm{A} 2$ & Work & $\mathrm{RL}<\mathrm{IL}$ & $P<.001$ & $25.2 \pm 3.7$ & vs & $34.2 \pm 6.6$ \\
\hline & & $\mathrm{A} 1$ & Power & $\mathrm{RL}>\mathrm{IL}$ & NS & $-1.2 \pm 0.4$ & vs & $-1.0 \pm 0.5$ \\
\hline & & Ecc & Work Stance & $\mathrm{RL}<\mathrm{IL}$ & NS & $1.2 \pm 0.5$ & vs & $1.7 \pm 0.5$ \\
\hline & Knee & K1 & Work & $\mathrm{RL}>\mathrm{IL}$ & NS & $-4.9 \pm 3.2$ & vs & $-4.6 \pm 3.8$ \\
\hline & & K2 & Work & $\mathrm{RL}>\mathrm{IL}$ & NS & $3.6 \pm 2.3$ & vs & $2.1 \pm 1.4$ \\
\hline & & K3 & Work & $\mathrm{RL}>\mathrm{IL}$ & NS & $-15.3 \pm 5.2$ & vs & $-9.8 \pm 5.4$ \\
\hline & & K4 & Work & $\mathrm{RL}>\mathrm{IL}$ & NS & $-9.5 \pm 1.9$ & vs & $-8.6 \pm 1.9$ \\
\hline & & K2 & Power & $\mathrm{RL}<\mathrm{IL}$ & $P<.05$ & $0.5 \pm 0.1$ & vs & $1.0 \pm 0.6$ \\
\hline & & K3 & Power & $\mathrm{RL}<\mathrm{IL}$ & NS & $-2.9 \pm 0.7$ & vs & $-4.4 \pm 1.3$ \\
\hline & Hip & $\mathrm{H} 1$ & Work & $\mathrm{RL}<\mathrm{IL}$ & $P<.05$ & $3.6 \pm 2.5$ & vs & $9.9 \pm 5.5$ \\
\hline & & $\mathrm{H} 2$ & Work & $\mathrm{RL}>\mathrm{IL}$ & $P<.02$ & $-8.3 \pm 3.3$ & vs & $-2.5 \pm 2.6$ \\
\hline & & H3 & Work & $\mathrm{RL}>\mathrm{IL}$ & NS & $9 \pm 1.9$ & vs & $7.3 \pm 2.8$ \\
\hline & & $\mathrm{H} 2$ & Power & $\mathrm{RL}<\mathrm{IL}$ & NS & $-0.9 \pm 0.2$ & vs & $-1.3 \pm 0.7$ \\
\hline & & $\mathrm{H} 3$ & Power & $\mathrm{RL}<\mathrm{IL}$ & NS & $0.9 \pm 0.3$ & vs & $1.0 \pm 0.4$ \\
\hline
\end{tabular}

Abbreviations: NS, non-significant; Conc, concentric; Ecc, eccentric

A total of 12 studies were included that studied TT amputation. One of the major alterations seen on the knee level of the amputated leg is the decreased amount of performed work during stance. These results reflect reduced involvement of the amputated leg in weight acceptance control. ${ }^{66}$ Previous authors stated that TT amputees tend to avoid large moments at the knee of the amputated leg during gait because these moments have the potential to generate moments between the residual limb and the socket. ${ }^{111}$ These moments 
can create areas of high pressure at the stump and are therefore minimized. The intact leg seems to compensate for the reduced involvement of the amputated leg by increasing the performed work at the knee. ${ }^{57,66}$ This could explain the increased incidence of osteoarthritis of the knee of the intact leg of TT amputation. ${ }^{112}$ On the hip level, major adaptations in performed work were seen in the amputated and intact legs. The concentric work of the hip extensors during the early stance phase is increased in the amputated and intact legs compared with a referent leg. ${ }^{57,110}$ These findings could indicate increased gluteus maximus activation. A modeling and simulation analysis of amputee walking showed increased gluteus maximus excitation in both the amputated and intact legs to transfer more energy to the trunk to provide forward progression. ${ }^{113}$ These results indicate that the glutei maximi of both legs compensate for the decreased push-off of the prosthetic ankle.

In contrast with the studies describing work, peak power values for the amputated leg during the $\mathrm{K} 1$ and $\mathrm{K} 2$ phases show no significant differences with the intact leg. A possible explanation could be that the peak values are similar but the duration of power generation or absorption is shorter. As a result, the area under the power profile is decreased, leading to lower work performance. Furthermore, some conflicting results were found between trials studying adults and children. A clear explanation for these findings is lacking. On the hip level, results of peak power values confirm the results of performed work. In addition, an increase in peak power of the amputated leg during the $\mathrm{H} 2$ phase was found compared with the intact leg. There is no clear explanation for this finding in TT amputation. Seroussi et al. ${ }^{48}$ speculate that in TF amputation, an increase in peak $\mathrm{H} 2$ power is the result of a lack of knee and hip flexion of the amputated leg, especially compared with the intact leg. This places the subjects' center of mass posterior with respect to the amputated leg. An increase in $\mathrm{H} 2$ power is thus necessary to pull the trunk over the amputated leg to prevent it from lagging too far behind. Whether this explanation also applies in TT amputation should be elucidated by future research.

Trials studying TT amputation used conventional (ie, SACH or SAFE) and dynamic (ie, Flex) feet. Because several studies used various types of feet and most of these trials presented overall figures, combining the results of different prosthetic components became inevitable. This may have biased the results found in this review because of a possible effect on kinetic variables. Schneider et al. ${ }^{79}$ found differences on the knee and hip levels with the SACH and Flex feet, described in the Results section of the present review. Comparison of energy storing and releasing (Carbon copy II, Flex, Seattle, and STEN) and more conventional feet $(\mathrm{SACH})$ showed no differences in peak power generation and absorption around the hip. ${ }^{114,115}$ Underwood et al. ${ }^{116}$ studied kinetic variables of the knee and hip with subjects wearing SAFE and Flex feet. No significant differences were found. Based on these results, it is difficult to explicitly state the influence of different prosthetic feet on the obtained results. In addition, we examined the forest plots to see whether there were different trends in outcome for conventional and dynamic feet. These trends, however, were not identified (Appendix 1). 
A total of 2 studies were included on the gait pattern of TF amputation. Results showed that on the hip level, the intact leg performed more work than a referent leg. The amputated leg had a tendency to perform more work than a referent leg, although this difference did not reach statistical significance. These results show remarkable similarities to the results of TT amputation. The relative differences, however, are larger in TF than TT amputation. A similar explanation is applicable: by increasing gluteus maximus excitation, progression is maintained. ${ }^{48}$ This finding is confirmed by an electromyogram study of Jaegers et al. ${ }^{2} \mathrm{TF}$ amputees used another adaptation strategy to compensate for the decreased push-off of the prosthetic ankle. During the A2 phase, significantly more work was performed by the intact leg than a referent leg. A third adaptation was seen during the $\mathrm{H} 2$ phase. A possible explanation for this finding is given by Seroussi et al. ${ }^{48}$ and described in this article.

The results of this systematic review must be generalized to other amputees with caution. The study populations mainly consisted of participants who were amputated for traumatic reasons. The total amputee population, however, is dominated by vascular amputees. This is of importance because traumatic amputees tend to have a higher walking velocity. From previous research, it is known that walking speed influences biomechanical variables. ${ }^{57,117}$

This review was confounded by a number of factors:

1. The methodologic quality of included studies was low. The studies scored low on the sub-scales external validity and internal validity (selection bias) in particular. It is known that these factors are often limited in observational research. ${ }^{118}$ Because the size and characteristics of the source population from which the sample was extracted were poorly described, the results of this meta-analysis and review are difficult to generalize. Furthermore, ambiguity existed in most studies whether the amputee subjects and controls were similar in all important respects, besides having an amputation. As a result, it is not known whether found differences could be attributed solely to the amputation.

2. For the comparison between the amputated and intact legs of TT amputees, the data of 4 different studies were pooled. The analysis that was carried out was based on the assumption that these data were independent. However, it can be assumed that these data were paired. A paired analysis was not possible because the covariance between the data of the amputated and the intact legs could not be calculated. This could have affected the results. See equation (2.1) for a paired analysis for an explanation:

$$
\bar{X}_{\text {pooled }}=\bar{X}_{1}+\bar{X}_{2}-2 * \text { covariance }
$$

It can be assumed that the amputated and the intact leg are negatively correlated because the intact leg seems to compensate for the deterioration of function of the amputated leg. This will lead to a negative covariance. When this is taken into account, 
a positive number will be added to the formula, thereby increasing the pooled variance. An increase in pooled variance results in an increase of the $P$ value. Therefore, the found $P$ values are in fact an underestimation of the actual $P$ values. However, because found significant differences showed $P$ values smaller than .01, we expected that these results would remain significant when the covariance was taken into account.

3. The trial by Nolan and Lees ${ }^{16}$ did not describe the used prosthetic feet. As stated, prosthetic feet influence biomechanical variables. Because the information regarding the used feet is lacking, it is unknown whether it was legitimate to pool the studies in the comparison of the intact leg and a referent leg in TT amputation.

Based on the reported confounding factors, a few guidelines for future research can be stated. First, future research should use more uniform outcome measures. Second, future studies should report the timing of peak values by using, for example, the categorization of Winter. ${ }^{13}$ Furthermore, future studies should clearly describe patient characteristics and used prostheses. Compliance with these 3 key factors will allow data pooling without raising questions about the legitimacy of the analysis.

\section{Conclusions}

In both TT and TF amputation, adaptations were seen in the amputated and intact legs. Most of the adaptation can be attributed to a reduced involvement of the amputated leg in weight acceptance and the loss of ankle plantar flexors. Based on these adaptations, it can be concluded that the amputated and intact legs are asymmetric in function. Striving toward gait symmetry based on the assumption that symmetrical gait is more functional seems therefore inappropriate. Finally, muscle groups were identified, primarily the hip extensors of both the amputated and intact legs, that compensate for the loss of sensorimotor function of the amputated body part. Rehabilitation programs could emphasize training these muscle groups, thereby enabling maximal adaptability. 


\section{Appendix}

\section{Joint Work, amputated-intact legs}

\section{H1 phase}

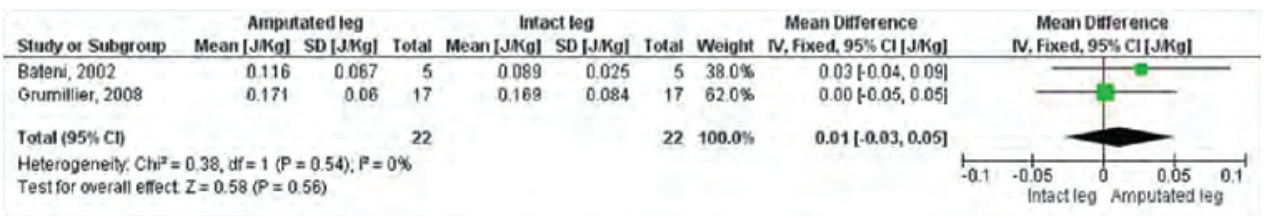

\section{Joint Power, amputated-intact legs}

\section{K1 phase}

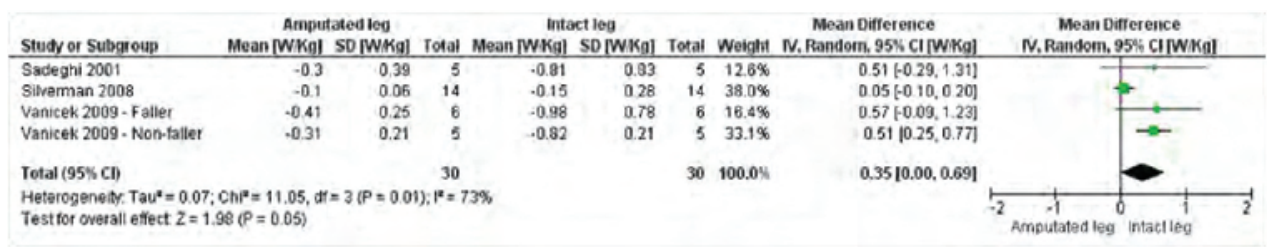

\section{K2 phase}

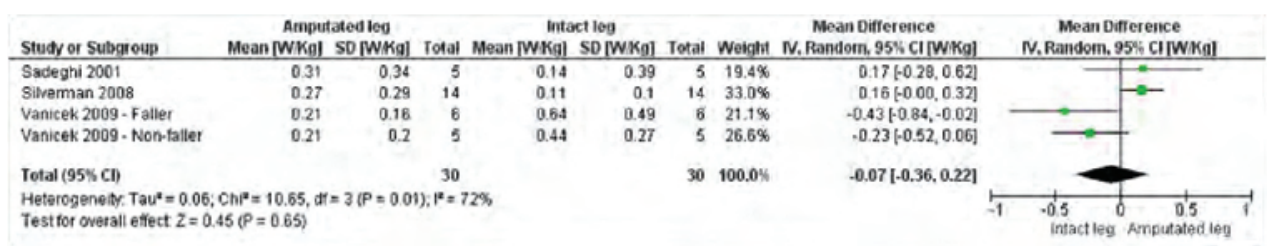

\section{K3 phase}

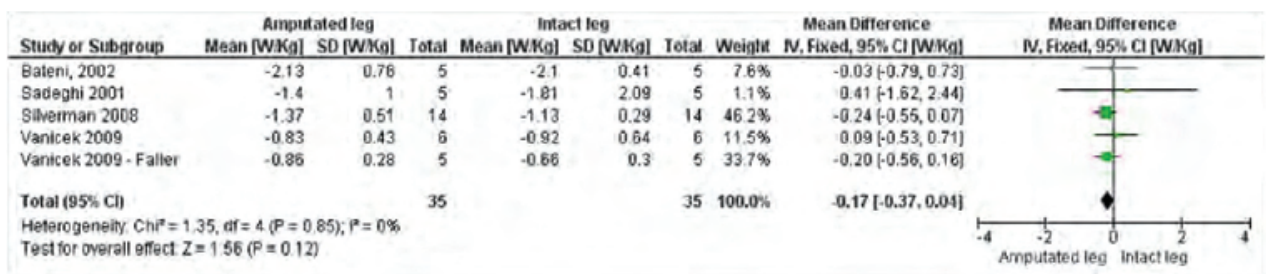




\section{K4 phase}

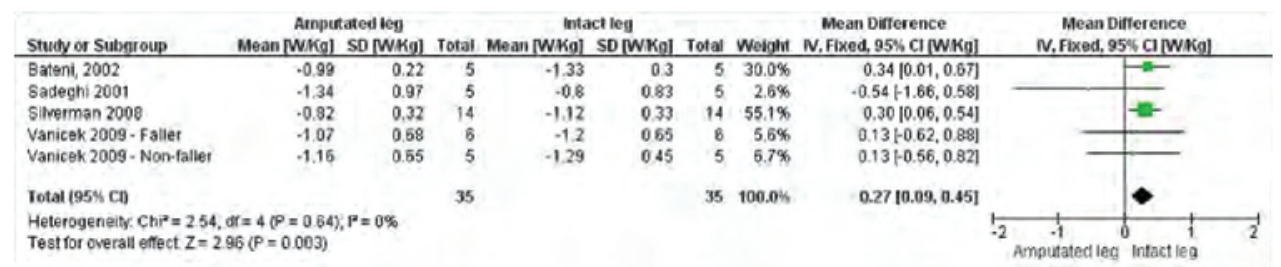

\section{H1 phase}

\begin{tabular}{|c|c|c|c|c|c|c|c|c|c|}
\hline Stuty or Subersoup & \multicolumn{2}{|c|}{ Ainputated ley } & \multicolumn{3}{|c|}{ Intact leg } & Total & Woight & $\begin{array}{l}\text { Mean Difference } \\
\text { N. Fixed, } 95 \% \mathrm{Cl}\left[\mathrm{WK}_{\mathrm{K}}\right]\end{array}$ & $\begin{array}{c}\text { Mean Difference } \\
\text { N, Fixed, } 95 \% \text { Cl [WKa] }\end{array}$ \\
\hline $\begin{array}{l}\text { Sadeghi } 2001 \\
\text { Silverman } 2000\end{array}$ & $\begin{array}{l}1.87 \\
0.85\end{array}$ & $\begin{array}{l}1.65 \\
0.34\end{array}$ & $\begin{array}{r}5 \\
14\end{array}$ & $\begin{array}{l}1.96 \\
0.93\end{array}$ & $\begin{array}{l}1.57 \\
027\end{array}$ & $\begin{array}{r}5 \\
14\end{array}$ & $\begin{array}{r}0.6 \% \\
45.0 \%\end{array}$ & $\begin{array}{l}-0.09+2.09,1.91\} \\
-0.08+-0.31,0.151\end{array}$ & \\
\hline Vanicek 2009 - Non-faller & 0.8 & 0.59 & 5 & 0.65 & 0.34 & 5 & $6.5 \%$ & $0.15 \nmid-0.45,0.75\}$ & \\
\hline Jotal $(95 \% \mathrm{Cl})$ & & & 35 & & & 35 & $100.0 \%$ & $0.03[-0.13,0.18]$ & \\
\hline
\end{tabular}

\section{H2 phase}

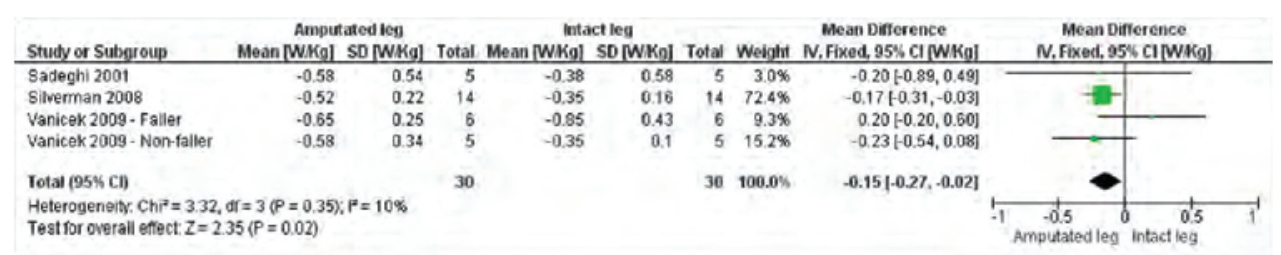

\section{H3 phase}

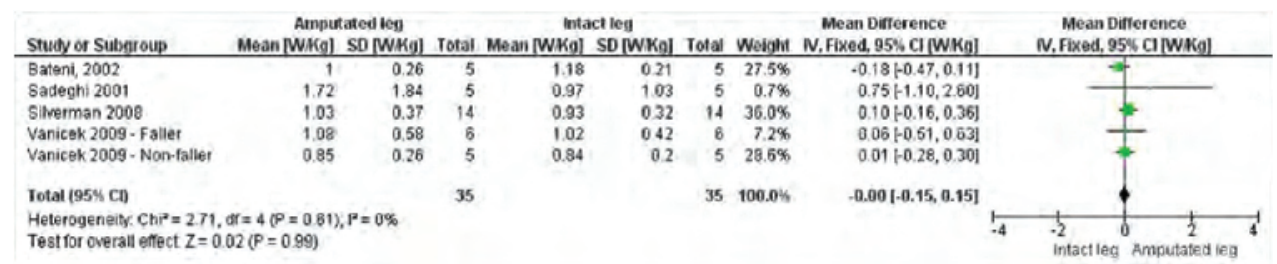

\section{Joint Power, amputated-referent legs}

\section{K4 phase}

Amputated leg Referent leg

Mean Difference Mean Difference Study or Subgroup Mean [WKg] SD [W.Kg] Total Mean [WKal SD [WKg] Total Weight N, Random, 95\% CIIWKg] N. Random, 95\% CI [WKo]

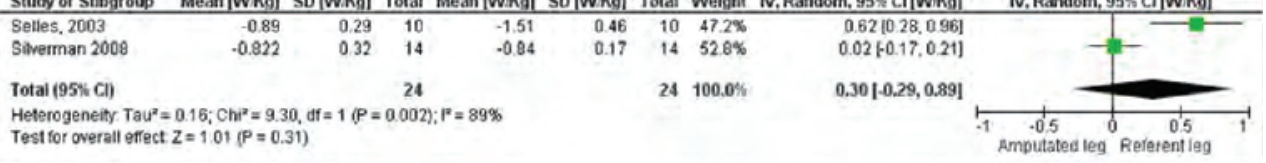




\section{Joint Power, intact-referent legs}

\section{A1 phase}

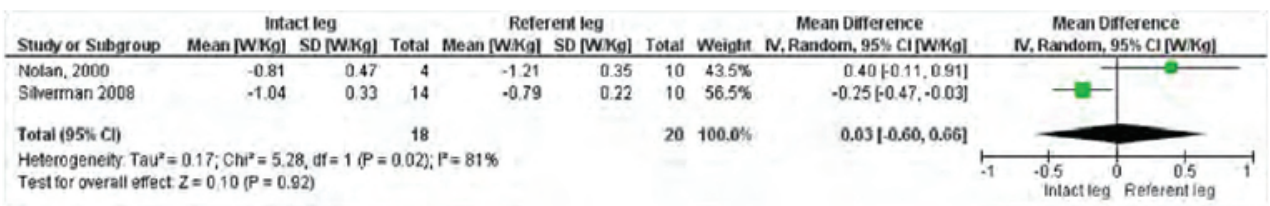

\section{A2 phase}

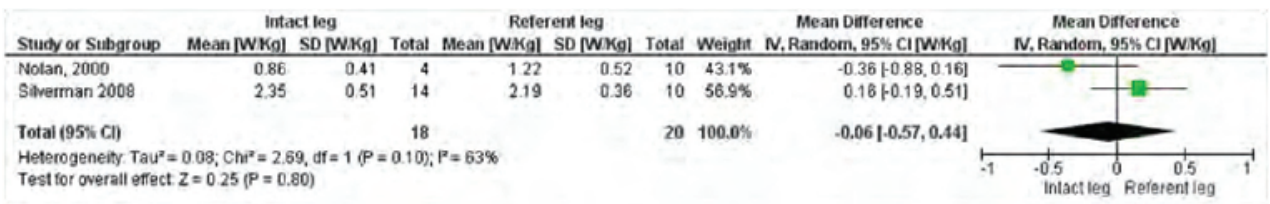

\section{K2 phase}

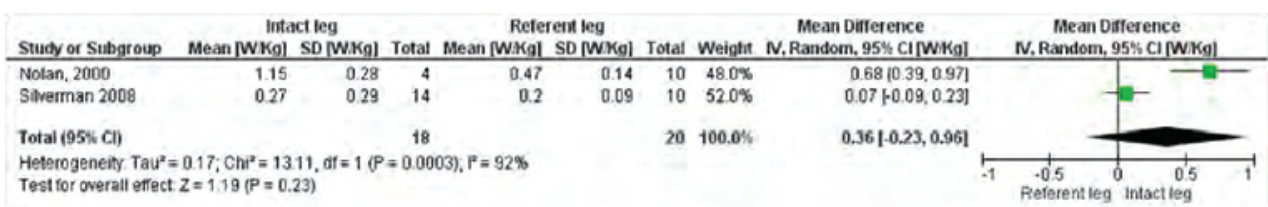

\section{K3 phase}

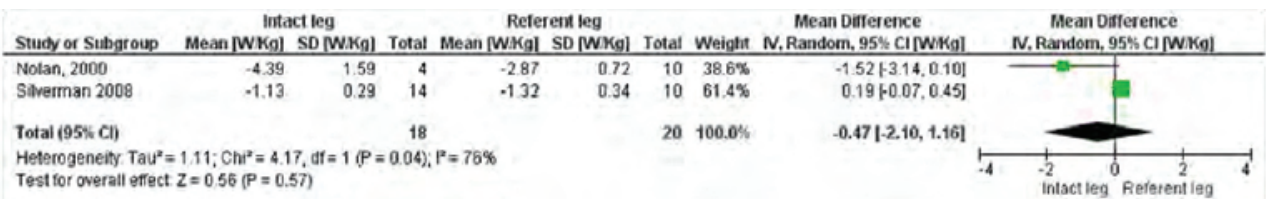

\section{H2 phase}

Intact leg Referent leg

Mean Difference Mean Difference

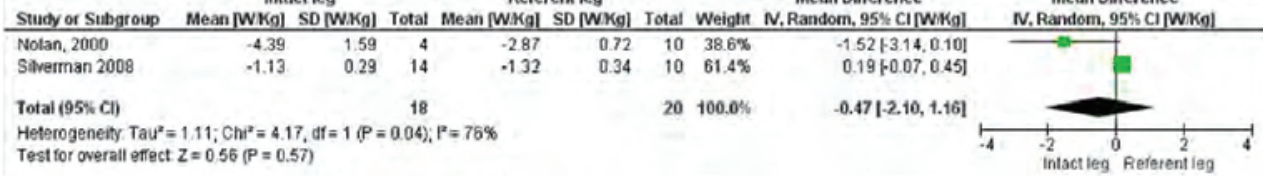




\section{H3 phase}

intact leg

Reterent leg

Mean Difference

Mean Difference Study or Subgroup Mean[WKg] SD [W.Kg] Total Mean [W:Kgl SD [WKof Notan, 2000
Silverman 2008

1.7
0.94 $\begin{array}{lll}0.4 & 4 & 0.85\end{array}$

0.93

$\begin{array}{lll}0.26 & 10 & 47.4 \% \\ 0.18 & 10 & 52.6 \%\end{array}$

Total (95\% Cl)

18

$20100.0 \%$

Heterogeneity Tau $=0.32 ; \mathrm{Ch}^{2}=12.31$, df $=1(\mathrm{P}=0.0005) ; \mathrm{P}=92 \%$

Test for overall effect $Z=0.97(P=0.33)$

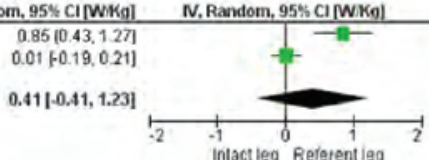




\section{Comparison of muscle activity}

patterns of transfemoral amputees and control subjects during walking

Wentink EC, Prinsen EC, Rietman JS, Veltink PH. Gait Posture 2014;39:391-6. 


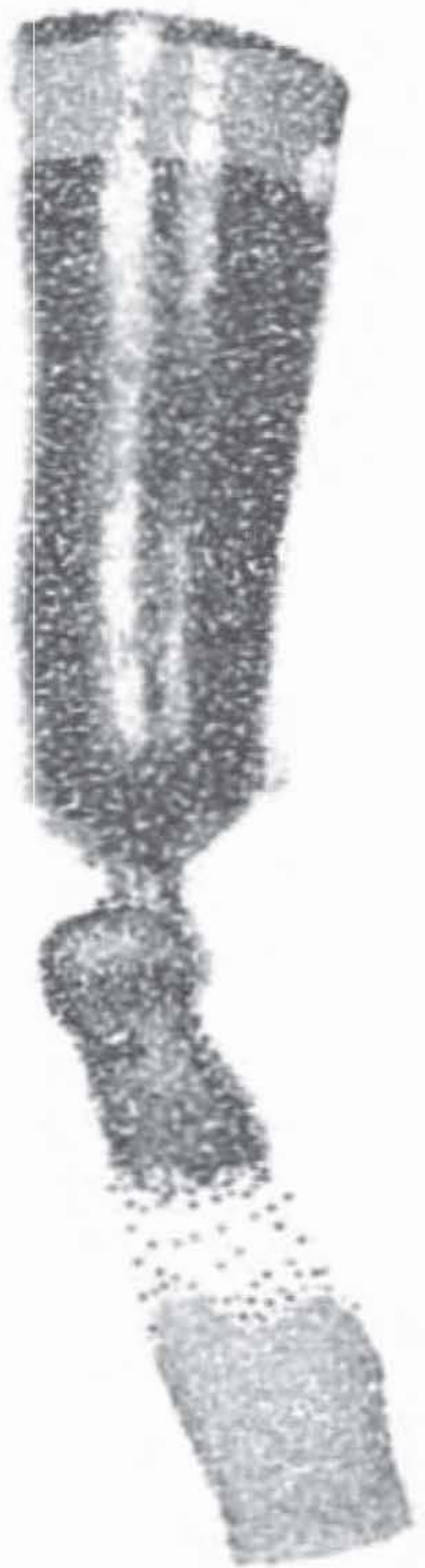




\section{ABSTRACT}

Background Only few studies have looked at electromyography (EMG) during prosthetic gait. Differences in EMG between normal and prosthetic gait for stance and swing phase were never separately analyzed. These differences can give valuable information if and how muscle activity changes in prosthetic gait.

Methods In this study EMG activity during gait of the upper leg muscles of six transfemoral amputees, measured inside their own socket, was compared to that of five controls. On and off timings for stance and swing phase were determined together with the level of coactivity and inter-subject variability.

Results and conclusions Gait phase changes in amputees mainly consisted of an increased double support phase preceding the prosthetic stance phase. For the subsequent (pre) swing phase the main differences were found in muscle activity patterns of the prosthetic limb, more muscles were active during this phase and/or with prolonged duration. The overall inter-subject variability was larger in amputees compared to controls.

Keywords EMG, Transfemoral amputee, Kinematics, Spatio-temporal data. 


\section{INTRODUCTION}

During rehabilitation transfemoral amputees (TFA) learn to adapt their gait pattern to walk with a prosthesis. Several of these adaptations are already known. During gait the stance phase of the amputated limb shortens compared to that of the intact limb. Therefore the swing phase is longer for the amputated limb. The double support phase elongates when the amputated limb becomes the stance limb and shortens when the intact limb becomes the stance limb. ${ }^{10,119}$ The comfortable walking speed of prosthetic walkers is also lower than in normal walking. 2,10,16,48 Kinematic data shows that transfemoral amputees lack plantar flexion power (push-off) at the prosthetic side. To facilitate forward propulsion they increase the work by the hip joint at the prosthetic and intact side and the plantar flexors at the intact side. ${ }^{16,48,120}$ Eccentric work at the hip of the intact side decreases with respect to normal gait. ${ }^{48}$ Joint power during concentric knee extension increases for the intact side, with respect to normal walking. ${ }^{16}$

However, little research has been performed on electromyography (EMG) during amputee gait. EMG of residual limb muscles of TFA may give valuable information on adaptations besides those that can be found using kinetic and spatio-temporal data. ${ }^{121}$ Some studies report increased and prolonged muscle activity in amputees during gait. ${ }^{2,109,120}$ Bae et al. ${ }^{109}$ concluded that the co-activation of the upper leg muscles of the intact limb in amputees was larger than in controls. Hong and Mun ${ }^{122}$ found that during gait the muscle activity of residual limb muscles in TFA is correlated to the socket pressure. If EMG patterns are different from that of controls this might indicate specific adaptations of amputees. Muscle activity per phase (stance and swing) can give more insight in the changes in themuscle activity patterns, how they change compared to normal walking and in the adaptations amputees make when walking with a prosthesis, besides kinematic changes.

In the current study we focus on muscle activity during the stance and swing phase of prosthetic gait. Do the muscle activity patterns of the prosthetic limb change and how do they change for the stance and swing phase, compared to normal gait? We intended to have as little interventions to the prosthesis and the subsequent walking pattern as possible. Therefore we measured EMG inside the socket, without modifications, of six amputees and compared this to data of five controls. Previous studies have shown that it is possible to measure EMG with acceptable quality inside the socket of amputees. ${ }^{2,121}$

From this data we determined if the timings of the muscle activity changed with respect to the different phases of gait compared to normal gait. We hypothesized that the general EMG patterns during walking are comparable to those in controls, but we expected to find differences related to specific adaptations in amputees. Three muscles at the contralateral lower leg were also measured to determine the adaptations at the intact lower leg. We determined how the inter-subject variability of amputees compares to that of controls. Spatio-temporal and kinematic data were also measured for gait phase determination and to relate the results to previous studies. 
Table 3.1: Characteristics of individuals with an amputation

\begin{tabular}{cccccccc}
\hline Subject & Type & Age (years) & $\begin{array}{c}\text { Reason } \\
\text { amputation }\end{array}$ & $\begin{array}{c}\text { Stump } \\
\text { length } \\
(\mathbf{m})\end{array}$ & Knee & Foot & $\begin{array}{c}\text { Time } \\
\text { (months) }\end{array}$ \\
\hline 1 & TKA & 52 & Traumatic & 0.56 & C-Leg & C-walk & 24 \\
2 & TKA & 46 & Traumatic & 0.59 & Rheo Knee & Vari-Flex EVO & 8 \\
3 & TKA & 29 & Dystrophy & 0.56 & C-Leg & 1 E56 & 5 \\
4 & TFA & 61 & Vascular & 0.41 & Total Knee & Elation & 5 \\
5 & TFA & 64 & Vascular & 0.41 & Total Knee & Elation & 6 \\
6 & TFA & 62 & Traumatic & 0.35 & C-Leg & 1 E56 & 133 \\
\hline
\end{tabular}

Abbreviations: TKA: through-knee amputation; TFA: transfemoral amptutation

\section{MethODS}

\section{Participants}

Eleven healthy subjects participated in the study, five controls and six unilateral amputees. All subjects were recruited between April and July 2011. Of the amputees there were three transfemoral amputees (TFA) and three through the knee amputees (TKA). An overview of the amputees can be found in Table 3.1. Inclusion criteria were: (1) have a unilateral TFA or TKA regardless of the reason for amputation, (2) be between 18 and 70 years old, and (3) be a prosthetic user able to walk independently with or without a walking aid (K-level 2, 3 and 4). The controls were on average aged 23 (range 21-27) and had no history of lower leg injuries, neurodegenerative diseases or any skin conditions. An informed consent was obtained before the experiments, and the study was approved by the local Ethics Committee. The institutional board, for the approval of the study is called METC Twente (or Medisch Ethische Toetsingscommissie Twente).

\section{Measurements}

EMG recording was performed on eight upper leg muscles in all subjects: $m$. gluteus maximus (GMa), m. gluteus medius (GMe), m. tensor fasciae latae (TFL), m. rectus femoris (RF), $m$. vastus lateralis (VL), $m$. biceps femoris (BF), $m$. semitendinosis (ST), $m$. adductor magnus (Add). In amputees these were measured on the residual limb, in controls these muscles were measured at one limb, which was alternated between dominant and nondominant limb. For amputees and controls this limb will be called the "prosthetic limb" and "mimicked prosthetic limb" respectively.

At the contralateral lower limb three more muscles were measured, the $\mathrm{m}$. tibialis anterior (TA), m. gastrocnemius medialis (GaM) and the $m$. soleus (Sol). For amputees and controls this limb will be called the "intact limb" and the "mimicked intact limb" respectively.

Electrodes were placed according to the SENIAM standards, ${ }^{123}$ by an experienced physical therapist. For the amputees the locations were approximated, but EMG was checked 
prior to the measurements by selective contraction of the muscle. ${ }^{123}$ On each muscle two self adhesive electrodes (Ambu, BRS) were placed as closely together as possible. EMG measurements were performed with a 16 bipolar channel Porti-system (TMSi, Oldenzaal, the Netherlands) at a sample frequency of $2048 \mathrm{~Hz}$, no prefiltering was applied.

Footswitches, placed mid-heel and under the first metatarsal head of each the foot, gave information about initial contact and initial swing. Footswitch data was registered with the Porti-system.

Kinematic data were measured $(100 \mathrm{~Hz})$ using inertial sensors from Xsens (Xsens, Enschede, the Netherlands), with 3D accelerometers, 3D gyroscopes and 3D magnetometers. Two inertial sensors were placed at the upper and lower (mimicked) prosthetic limb. Subjects wore their own low-heeled shoes.

To synchronize EMG, footswitches and inertial sensors a synchronization pulse (sync) was given at the start and end of each measurement which was visible in all data sets.

\section{Procedures}

For the experiments the subjects were asked to walk at a self selected walking speed. After data recording was started, the sync was pressed and subjects started walking. After five steps they were asked to stop, turn around, wait 2-3 seconds, press the sync and walk back; this constituted one trial. Four trials were performed in all subjects.

\section{Data analysis}

From the footswitch data the timings of initial contact (IC), terminal stance, initial swing and loading response of each limb were determined. ${ }^{3}$ Foot switches were used to extract the spatio-temporal information. Full strides were cut from the EMG and inertial sensor data, from IC to IC of the (mimicked) prosthetic limb. Strides with gait initiation or termination were excluded. All strides per subject were aligned at IC of the (mimicked) prosthetic limb.

\section{Inertial sensor data}

The inertial sensor data was expressed in the body coordinate system based on a sensorsegment calibration procedure as described by Wentink et al. ${ }^{124}$ This data was subsequently low-pass filtered at $10 \mathrm{~Hz}$ with a second order, butterworth filter. From the calibrated inertial sensor data the knee angle, hip adduction and abduction are calculated using accelerometer and gyroscope data by the method described by Watanabe et al. ${ }^{125}$

\section{EMG data}

EMG data was first high pass filtered at $10 \mathrm{~Hz}$ and subsequently low pass filtered at $500 \mathrm{~Hz}$, both with a $2^{\text {nd }}$ order Butterworth filter. In Figures 3.1, 3.2, 3.3, and 3.4 the ensemble averages of all amputees and controls separately are provided, including the raw and filtered data of one subject, of one trial for all muscles. In Figure 3.1 an example of filtered data is presented. For on and off detection the data was rectified and integrated (IA) in a window 

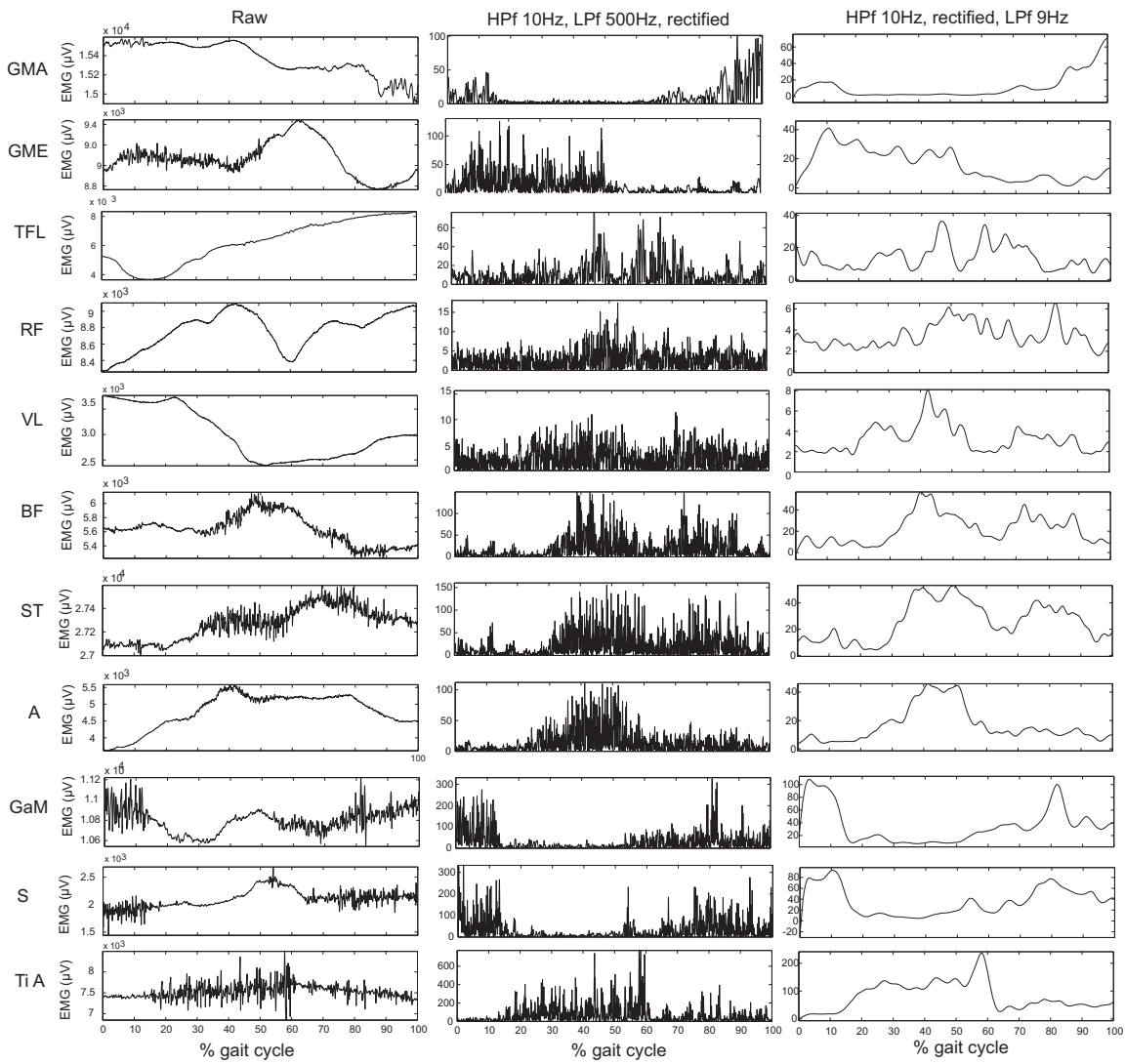

Figure 3.1: Raw EMG data.

An example of filtered activity of all upper and lower leg muscles, of one subject during one trial. The row on the left shows the raw data of the trial during one gait cycle. The middle row shows the high pass filtered (HPf), rectified and low-pass filtered (LPf) data of the same trial and the right hand row shows the linear envelope HPf at $10 \mathrm{~Hz}$, rectified and $\mathrm{LPf}$ at $9 \mathrm{~Hz}$.

of 20 samples, a post processor of four windows was used. The threshold for on/off detection was determined per muscle. A period of rest activity was selected, and the mean IA value of this period plus three times the standard deviation was used as threshold for onset and termination of muscle activity. ${ }^{126-128}$ For each muscle, each stride and each subject the on/off timings were calculated. These timings were averaged per subject, to get the on and off timings per muscle, per subject.

The stance and swing phase of the (mimicked) prosthetic limb were calculated per subject and expressed as percentage of the total stride time. Using the average muscle on/off timings per subject, we subsequently calculated for which percentage of the stance or swing phase the muscles were active. These were subsequently averaged for the controls and the amputees. Differences between controls and amputees were analyzed using a Kruskal- 
Biceps Femoris EMG
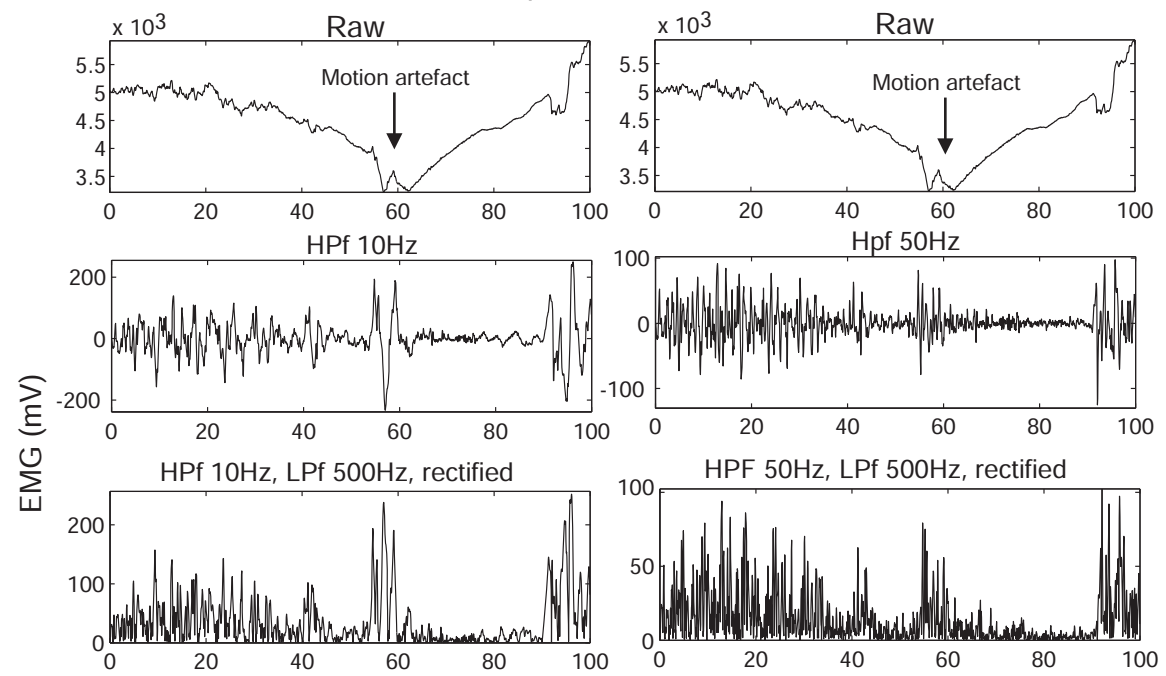

HPf $10 \mathrm{~Hz}$, rectified, LPf $9 \mathrm{~Hz}$
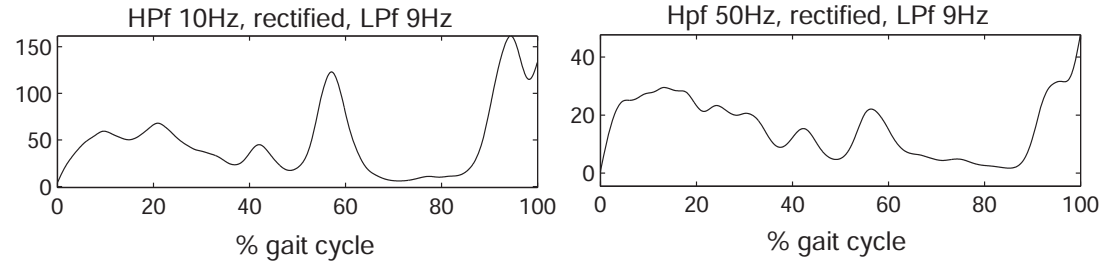

Figure 3.2: Motion artifacts.

An example of one amputee of the Biceps Femoris EMG with a motion artifact. On the left the data is high-pass filtered at $10 \mathrm{~Hz}$, as in all trials without motion artifacts, but this does not remove the artifacts. On the right the data is high-pass filtered at $50 \mathrm{~Hz}$, which did remove the motion artifact. Trials with this type of artifact that was removed by a $50 \mathrm{~Hz} \mathrm{HP}$ filter, but not by a $10 \mathrm{~Hz} \mathrm{Hp}$ filter were removed from the data. Most trials did not show this type of artifact and therefore the trials with artifacts were removed from the analysis and the original filtering was used.

Wallis test. The level of alpha was set at 0.05 .

The inter-subject variability of the EMG data was determined using the variance ratio (VR) for each subject and muscle for the stance and the swing phase. ${ }^{128,129}$ The VR is the variance of the data between gait cycles normalized to the total variance, whereby 0 indicates a low variance and 1 a high variance. Differences between the controls and amputees were analyzed using the Mann-Whitney-Wilcoxon test. ${ }^{128}$ The standard error of the mean (SEM), was calculated using equation (3.1), where $\mathrm{N}$ is the number of subjects per group. ${ }^{130}$

$$
S E M=\frac{S D_{\% \text { on } / \text { off-time }}}{\sqrt{N}}
$$



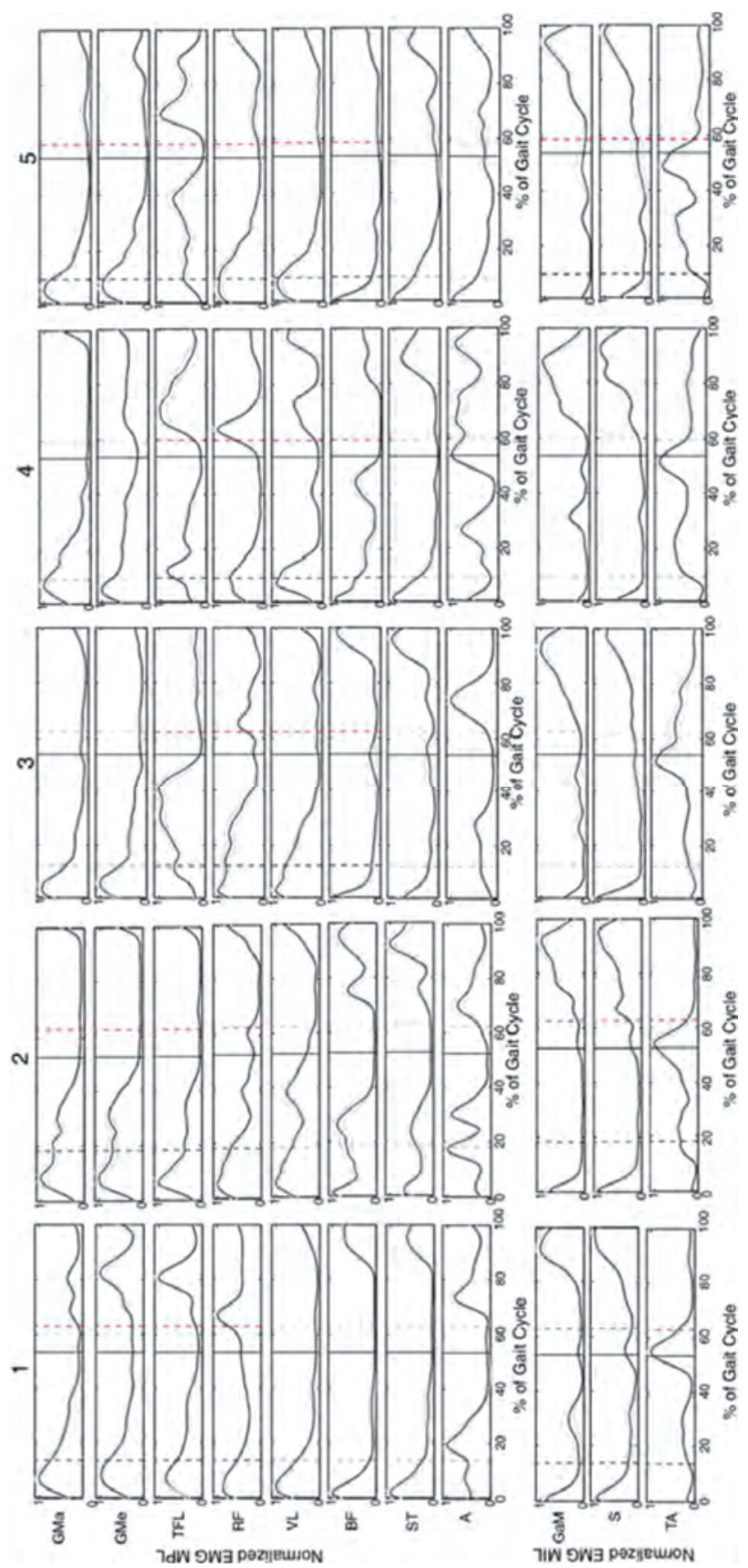

Figure 3.3: Ensemble averages controls.

The ensemble averages of each of the controls averaged over all trails (20) for each of the measured muscles. The black dashed line represents initial swing of the MIL, the black solid line initial contact of the MIL and the red dashed line initial swing of the MPL. (MPL = mimicked prosthetic limb, MIL = mimicked intact limb). 

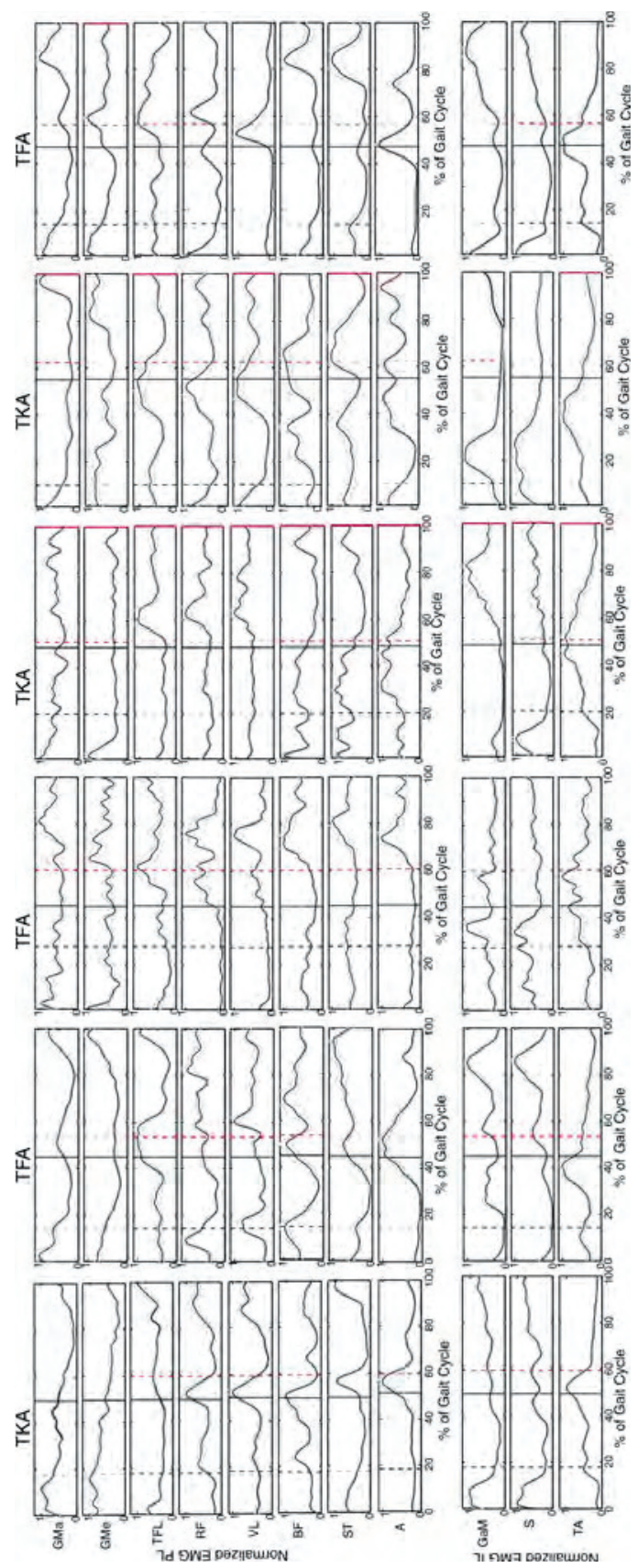

Figure 3.4: Ensemble averages amputees.

The ensemble averages of each of the amputees averaged over all trails (20) for each of the measured muscles. The amputee data is deliberately not placed in the order of amputees seen in table 3.1, to prevent matching of data and subjects. The black dashed line represents initial swing of the IL, the black solid line initial contact of the IL and the red dashed line initial swing of the PL. (PL = prosthetic limb, IL = intact limb). 
Table 3.2: Gait phase durations

\begin{tabular}{lccc}
\hline Phase & Controls value (SD) & Amputees value (SD) & Statistical note \\
\hline Stride duration & $1256 \mathrm{~ms}(72)$ & $1468 \mathrm{~ms}(307)$ & Not significant \\
Total stance (M)PL & $61 \%(2)$ & $55 \%(9)$ & $P=\mathbf{0 . 0 1 0}$ \\
Total stance (M)IL & $60 \%(3)$ & $71 \%(6)$ & $P=\mathbf{0 . 0 0 8}$ \\
Swing (M)PL & $39 \%(2)$ & $45 \%(3)$ & $P=\mathbf{0 . 0 1 0}$ \\
Swing (M)IL & $40 \%(3)$ & $29 \%(3)$ & $P=\mathbf{0 . 0 0 8}$ \\
DLS (M)PL & $11 \%(1)$ & $20 \%(9)$ & $P=\mathbf{0 . 0 4 5}$ \\
DLS (M)IL & $10 \%(3)$ & $7 \%(3)$ & Not significant \\
\hline
\end{tabular}

Abbreviations: C: controls; A: amputees; (M)IL: (mimicked) intact limb; (M)PL:

(mimicked) prosthetic limb; SLS: single limb support; DLS: double limb support

\section{RESULTS}

\section{Spatiotemporal data}

In Table 3.2 the average duration of a stride and the different gait phases in percentages of a stride are presented. A shift of all phases can be seen for amputees, Figure 3.5. For amputees the relative duration of the stance phase of intact limb, the prosthetic swing phase and the (first) double support phase before the prosthetic single stance phase are significantly increased compared to controls. The (second) double support phase of amputees before the prosthetic swing phase, is shortened but not statistically significant. Compared to the total stance phase, this "second" double stance phase is equal for both controls and amputees (15\%). No differences were found between TFA and TKA, nor between mechanical and micro-processor-controlled (MPC) knees.

\section{Kinematic data}

Table 3.3 shows the movements in degrees around the hip and knee the movement patterns around the hip and knee joints. Hip adduction and abduction are significantly reduced in amputees compared to controls. Knee flexion during stance as well as swing is also significantly reduced in amputees. No differences were found between TFA and TKA, nor were they found between mechanical and MPC knees.

\section{EMG data}

In Figure 3.1 a sample trial of EMG measured inside (upper leg) and outside (lower leg) the socket are provided of one subject. Both EMG measured inside and outside the socket shows to be of similar quality, without motion artifacts. 24 complete steps were measured in each subject, per subject at least 20 steps were included in the analysis. No steps were excluded from the controls. From the amputee data four subjects showed motion artifacts (see example in Figure 3.2) in maximally three trials in one or more muscles. In one other amputee four trials were excluded due to missing footswitch data. Figures 3.3 and 3.4 show 


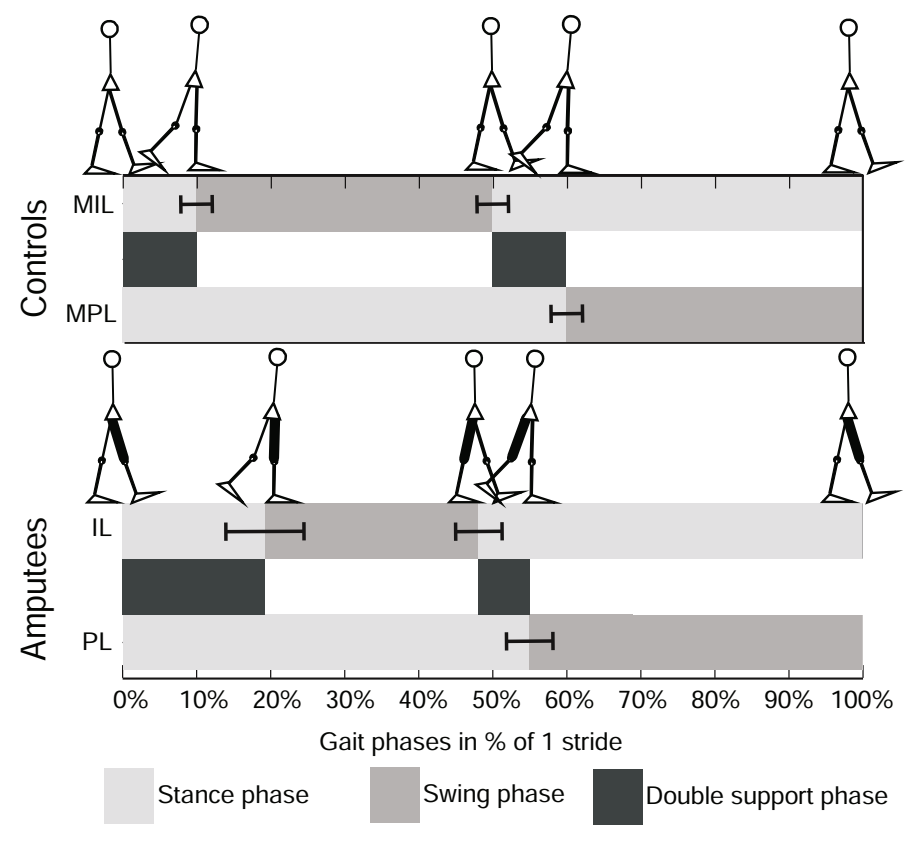

Figure 3.5: Gait phases.

Gait phases for controls and amputees, as percentages of one full stride. In black the swing phase, in light grey the stance phase and in dark gray the double support phases. The whiskers represent one SD.

the ensemble averages of each of the controls and amputees respectively. Figure 3.6 shows the timings of the upper leg muscles of the (mimicked) prosthetic limb for amputees and controls as percentages of the stance and swing phases of the (mimicked) prosthetic limb. Data of the lower leg muscles are from the contralateral limb.

\section{Stance phase}

During the (prosthetic) stance phase, the GMa of the amputee group is active for a longer period after initial contact. All other upper leg muscles are active for a similar or shorter period. In the amputee group, some muscles, become active a second time during stance; the TFL, VL, BF, ST and Add. In the controls this second phase of activity during stance for these muscles is not seen. The first period of activity shown for the RF is probably crosstalk by the VL. ${ }^{131,132}$ The RF becomes active just before terminal stance in controls, but shows no activity in this phase in the amputee group.

During the stance phase of the intact limb, activity of the GaM starts around the same time in controls and amputees, Sol activity of amputees starts a little earlier. The activity of the TiA in amputees continues longer during the stance phase of the intact limb, compared to controls. No significant differences were found between the activation patterns of the stance phase between controls and amputees. 


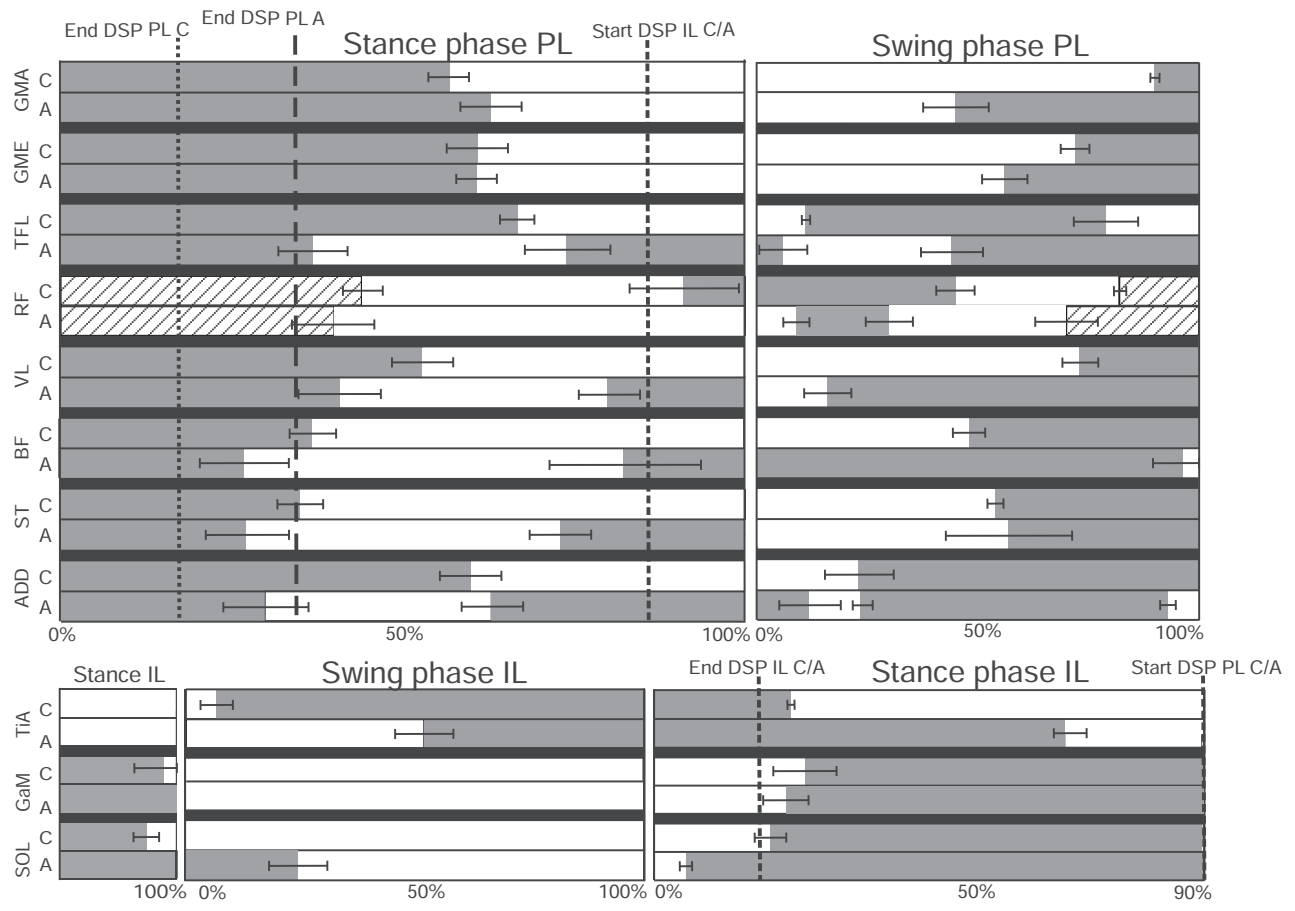

Figure 3.6: Overview muscle activity.

The average muscle activity of all muscles, for controls and amputees, as a percentage of the (mimicked) prosthetic stance and swing phase. In dark grey the muscle is "on", in white it is "off" and in hatched white periods of possible cross-talk. For the stance phase $0 \%$ is IC of the (mimicked) prosthetic limb and $100 \%$ is initial swing of the (mimicked) prosthetic limb. The end of the first double stance phase (DLS PL) before (mimicked) prosthetic stance (PL), of controls and amputees are indicated. The start of the second double limb support (DLS IL) is also indicated, which is equal for controls and amputees. For the swing phase, $0 \%$ is initial swing of the (mimicked) prosthetic limb and $100 \%$ is IC of the (mimicked) prosthetic limb. The whiskers show the standard error of the mean (SEM). All upper leg muscles are measured at the (mimicked) prosthetic limb and all lower leg muscles at the contralateral (mimicked) intact limb. Lower leg activity is scaled similarly, but to the phases of the intact leg. Hereby part of the stance phase (the DLS PL) is placed at the left hand side of the figure. 
Table 3.3: Joint angles of the hip and knee

\begin{tabular}{lccccc}
\hline & \multicolumn{2}{c}{ Controls } & \multicolumn{2}{c}{ Amputees } & \multirow{2}{*}{ Statistical note } \\
\cline { 2 - 5 } Variables & Average (SD) & Range & Average (SD) & Range & \\
\hline Max Hip flexion & $28^{\circ}(5)$ & $19^{\circ}-37^{\circ}$ & $26^{\circ}(12)$ & $15^{\circ}-43^{\circ}$ & Not significant \\
Max Hip extension & $13^{\circ}(5)$ & $6^{\circ}-20^{\circ}$ & $15^{\circ}(8)$ & $2^{\circ}-25^{\circ}$ & Not significant \\
Max Hip adduction & $9^{\circ}(1)$ & $8^{\circ}-10^{\circ}$ & $6^{\circ}(2)$ & $3^{\circ}-9^{\circ}$ & $P=0.006$ \\
Max Hip abduction & $11^{\circ}(2)$ & $9^{\circ}-14^{\circ}$ & $7^{\circ}(3)$ & $3^{\circ}-11^{\circ}$ & $P=0.002$ \\
Max Hip abduction & $11^{\circ}(2)$ & $9^{\circ}-14^{\circ}$ & $7^{\circ}(3)$ & $3^{\circ}-11^{\circ}$ & $P=0.002$ \\
Max Knee flexion stance & $13^{\circ}(4)$ & $8^{\circ}-18^{\circ}$ & $4^{\circ}(3)$ & $0^{\circ}-6^{\circ}$ & $P=0.006$ \\
Max Knee flexion swing & $57^{\circ}(6)$ & $47^{\circ}-68^{\circ}$ & $42^{\circ}(13)$ & $22^{\circ}-55^{\circ}$ & $P=0.029$ \\
\hline
\end{tabular}

Abbreviations: C: controls; A: amputees

\section{Swing phase}

The differences in muscle activity during the swing phase are larger than for the stance phase. The GMe and GMa of the amputees become active in the second half of the swing phase, whereas in the controls they become active at the end of the swing phase. This increased duration of activation is also seen for the RF, the VL and the BF. The TFL is also active at the transition from stance to swing, and has a later "second" activity onset at the end of the swing phase. The Add is active in amputees before initial swing and at the beginning of the swing phase, which is not the case in controls.

TiA activity during the swing phase of the intact limb starts later in amputees compared to controls. No differences are seen in GaM activity during the swing phase of the intact limb, but the Sol of the amputees shows activity during the first part of the swing phase, where controls do not show this activity. No significant differences were found between the activation patterns of the swing phase of controls and amputees.

\section{Variability}

The overall inter-subject variability of the EMG data of amputees is significantly lower $(P=$ 0.011 ) than that of controls (figure 3.7). The variability per muscle however is in none of the muscles significantly different. The mean VR of controls ranged from 0.30 to 0.48 , for amputees this range was 0.47 to 0.64 . The SEM of the EMG data is around $3 \%$ of the stance and swing phase for controls, for amputees this is a little higher, around $4 \%$ of the stance and swing phase with some outliers at $12-14 \%$.

\section{DISCUSSION}

\section{Kinematic and spatio-temporal data}

The kinematic data showed that the stance phase duration of the intact limb increases and the prosthetic swing phase duration also increases in amputees. This coincides with the gen- 


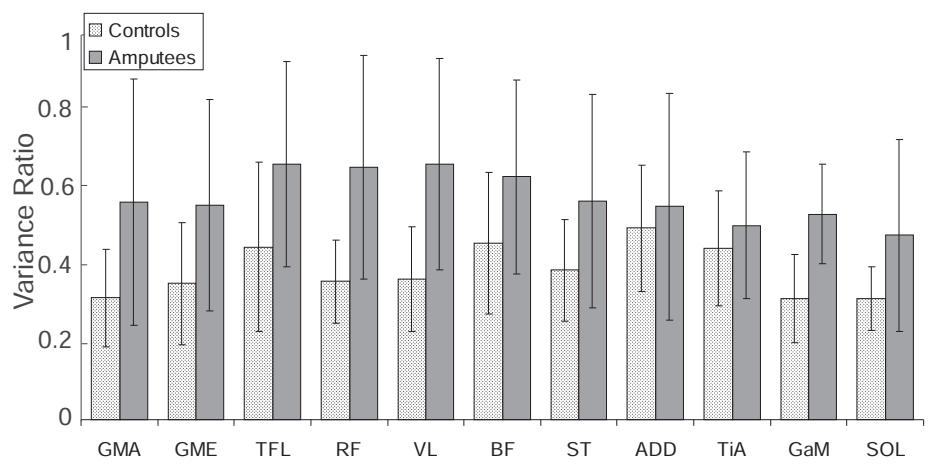

Figure 3.7: The variance ratios of all muscles of controls and amputees. Whiskers give one SD.

eral concept that amputees tend to stand longer on their intact limb than on their prosthetic limb, which has also been found in other studies. ${ }^{10,119}$ Knee flexion during initial stance differs. Controls show a knee flexion of up to $18^{\circ}$, in amputees this is only $4^{\circ}$ even though all amputees had a prosthetic knee which allows knee flexion during stance. This lack of knee flexion might indicate that amputees are not comfortable using knee flexion during initial stance of the prosthetic limb, which may be caused by a lack of trust or experience in using the MPC knee to the full potential. Hip adduction and abduction are also reduced in amputees, which was also reported by Jaegers et al. ${ }^{10}$ The reduction in hip adduction has most likely only a small effect on the walking pattern, as it is only a few degrees less than in controls. During normal single limb stance a small amount of adduction is seen, to ensure that the center of mass does not have to move laterally to keep it above the supporting surface. However when amputees are in prosthetic single limb support they will not bring their CoM above their support surface, but keep it more medially. This can be explained by the fact that in the frontal plane they have little opportunity to correct themselves, too much lateral motion will cause a fall. This reduces the need for adduction in stance. The reduced abduction may change the walking pattern of amputees. Hip abduction is used to "shorten" the leg to ease foot clearance during the transition from the stance to the swing phase. However TFA generally find it more difficult to perform hip abduction, which makes foot clearance more difficult. The reduction in hip abduction may create the need for more adaptations from the intact limb, for instance increased plantar flexion during single intact limb support (vaulting).

\section{EMG}

The differences found in muscle activity between prosthetic users and controls are mainly present in the (pre)swing phase. Muscle activity of controls resembles hat of previous studies, although muscles show activity for a longer period of time. ${ }^{3,133}$ This may be due to the onset detection method, but the exact methods used in the previous studies were not described. Therefore it is hard to find a clear explanation for this discrepancy. Visual com- 
parison of raw and filtered EMG data showed comparable EMG quality between controls and amputees.

\section{Stance phase}

When the gait stance and swing phases are compared separately, muscles in amputees do not seem to be active for much longer than in controls. At the end of the stance phase a period of activity is seen in most of the upper leg muscles, starting around the beginning of the second double support phase. This may be the mechanism by amputees to increase socket fitting at the end of the stance phase, to prepare for lifting of the prosthesis in the swing phase. ${ }^{122}$ Lower leg muscles of the contralateral side show a prolonged activity during stance. This increased activity could be used to ease foot clearance, ankle plantar flexion of the intact limb is used to virtually lengthen the intact limb. The prolonged activity can also be explained by the increased push-off needed from the intact limb, to propel the body forwards, to compensate for the lack of push-off on the prosthetic side. This coincides with the kinetic data, which showed increased work at the hip and plantar flexors of the intact limb. ${ }^{16,48,120}$

\section{Swing phase}

Some of the upper leg muscles of the amputees, the $B F$ and the $V L$, remain active for almost the complete swing phase. The other muscles all become active again at the end of the swing phase to prepare for initial contact. These muscles show an earlier activity onset than in controls, which may be explained by the walking strategy of amputees. Many amputees try to fully extend the knee to ensure it is locked at the end of the swing phase which is also confirmed by the reduced knee flexion during initial stance.

Our results resemble the results presented by Jaegers et al., ${ }^{2}$ as far as they can be compared. They only showed muscle activity for the complete gait cycle and no exact onset timings were calculated. They also reported activity before initial swing and found differences between subjects with an amputation in the proximal or distal half of the upper leg. ${ }^{2}$ In the current study all amputees were amputated at the distal half of the upper leg. In some muscles the activity is slightly longer or shorter compared to Jaegers et al. ${ }^{2}$ This can be due to the separated stance and swing phases in the current study and due to different approaches in detection times.

TFA showed a different activation pattern in some phases of the gait cycle, which shows that they adapt to their new prosthetic situation. Although the results show that consistent muscle activity can be measured inside the socket of TFA, the usability for prosthetic control is questionable. Variability between the amputees is higher, although patterns within the amputees are consistent. Although muscle activity patterns can change due to the disturbed anatomy by the amputation and by use of the prosthesis, training may allow TFA to learn new walking patterns which in turn may need adaptations in the muscle activity patterns to control a prosthesis. ${ }^{121}$ 


\section{Variability}

The overall inter-subject variability of the EMG data from the amputees was significantly higher than that from controls. VRs per muscle were however for none of the muscles significantly different. Granata et al. ${ }^{134}$ reported VRs in healthy adults between 0.17 and 0.27 , although they can go up to 0.76 in healthy adults. ${ }^{135}$ The main reason for a higher VR in amputees (up to 0.64 ) is most likely the lower walking speed. ${ }^{136}$

Many of the muscles in the upper leg of the amputees are cleaved. The electrodes were placed and tested for activity according to the SENIAM standards. However, due to the amputation some muscles may have a different location and the location of the electrodes may not have been ideal. Rotations in the socket may also affect the position of the electrodes with respect to the muscle. Poor socket fitting will affect the repeatability of the signal, this will induce more noise and the prosthetic usermay show more muscle activity to properly control or fit the prosthesis. One subject complained of non-optimal socket fitting as it was too large. This subject had a higher VR. None of the subjects complained about the EMG electrodes, they did not seem to effect the socket fitting. However, this does not explain the increased VR in the lower leg muscles of the contralateral limb and the hip muscles. This might indicate that the walking pattern of amputees is less consistent than that of controls. The standard deviations within amputees for spatio-temporal and kinematic data were also larger than in controls.

\section{Methodological considerations}

The amputees were a mixed group. No inclusion criteria for type of amputation or time since amputation were added. The average age (52.3) was larger than that of the controls (23). Previous studies have shown that aging may affect the spinal cord activity, walking speed and cause a higher spread in muscle activation. ${ }^{133,137}$ Also three prosthetic users were only prosthetic users for 5-6 month, of whom the EMG pattern may still change over time. Two of them were the elderly subjects with vascular diseases. One of these subjects also walked with a walking aid, which may also effect the muscle activity, ${ }^{138}$ both subjects had higher VRs. Nevertheless, even with the large variability in the group, no large deviations were seen in the EMG patterns of these subjects.

A more homogeneous and larger group of amputees with similar prosthesismay reduce the variability between the subjects. We did not find any obvious differences between the different knees, but this may also be caused by the low number of amputees. Including amputees with a short residual limb as Jaegers et al. ${ }^{2,10}$ did, can be an interesting addition. Measurements were performed inside the socket of the amputees. The residual limbsocket interface may have lead to increased motion artifacts, compared to using an experimental socket with build in EMG sensors. Data were checked for these artifacts. It occurred only occasionally during initial contact or initial swing that these artifacts were not removed by filtering. Trials with motion artifacts were removed, but this still allowed at least 20 steps to be included per subject. No motion artifacts were found in controls. Although we measured 
EMG inside the socket with reasonable quality, we did not test the reliability and validity compared to EMG measured using an experimental socket. We placed electrodes and performed EMG measurements according to the SENIAM standards, which are based on normal anatomy. No information on actual muscle locations were available, for instance from MRI. After electrode placement muscle activity was checked using selective muscle contraction. Only occasionally electrodes needed to be replaced, for a better location with respect to the muscle belly, but never more than 2-4 cm from the original placement. Therefore normal anatomy was assumed in amputees, with respect to cross-talk. Surface EMG was used for ease of electrode placement and comfort to the patient. Intramuscular EMG may have given less crosstalk and possibly more information on specific muscle activity, but it is impossible to use in the own socket of the amputees and very uncomfortable to the patient.

\section{Conclusion}

In amputees the double support phase before the prosthetic stance phase increases significantly and the prosthetic swing phase shortens. EMG patterns mainly differ at the end of the stance phase and in the swing phase. These changes can explain the changes in walking strategy, but are likely also required to improve socket fitting. In this study EMG was measured inside the socket of amputees, and the data showed to be of comparable quality compared to that of controls. Variance within each amputee is higher than in controls, but variability in the kinematic data between the amputees is also higher. The increased variance may mainly be caused by the variability in walking pattern and cleavage of muscles. 


\section{The infuence of a user-adaptive prosthetic knee across varying walking speeds:}

A randomized cross-over trial

Prinsen EC, Nederhand MJ, Sveinsdóttir H, Prins MR, van der Meer F, Koopman HFJM, Rietman JS. Accepted for publication in Gait and Posture. 


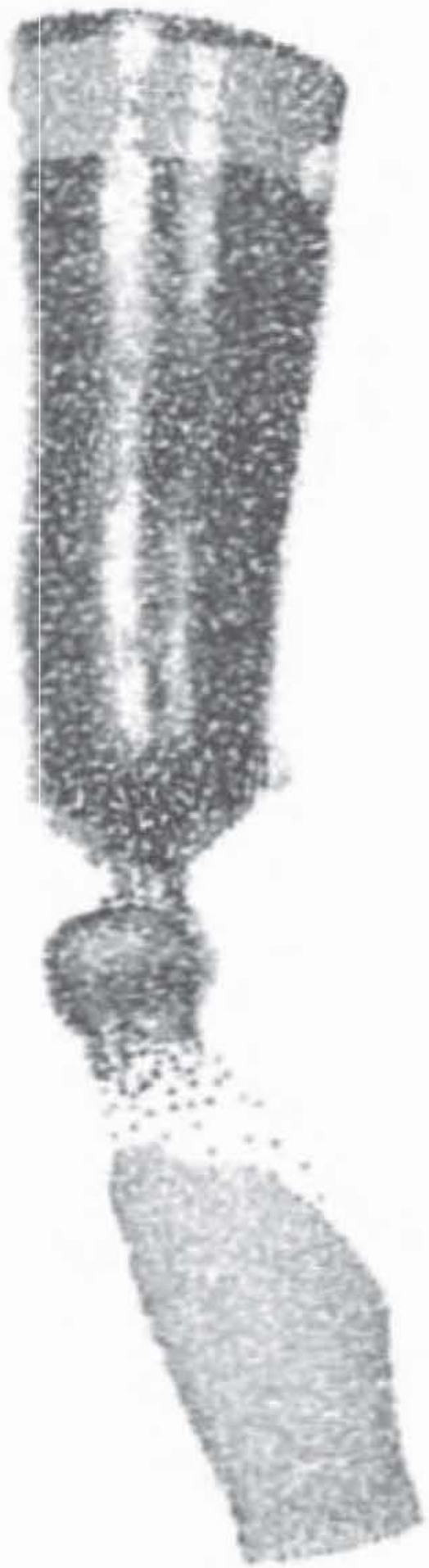




\section{ABstract}

Previously conducted trials comparing the gait pattern of individuals with a transfemoral amputation using a user-adaptive and a non-microprocessor-controlled prosthetic knee (NMPK) found mixed and conflicting results. Few trials, however, have compared useradaptive to non-adaptive prosthetic knees across different walking speeds. Because of the ability of variable damping, the effect of user-adaptive knees might be more pronounced at lower or higher walking speeds. Our aim was to compare the Rheo Knee II (a user-adaptive prosthetic knee) with NMPKs across varying walking speeds. In addition, we studied compensatory mechanisms associated with non-optimal prosthetic knee kinematics, such as intact ankle vaulting and vertical acceleration of the pelvis. Nine persons with a transfemoral amputation or knee disarticulation were included and measured with their own NMPK and with the Rheo Knee II. Measurements were performed at three walking speeds: preferred walking speed, $70 \%$ preferred walking speed and $115 \%$ preferred walking speed. No differences on peak prosthetic knee flexion during swing were found between prosthetic knee conditions. In addition, prosthetic knee flexion increased significantly with walking speed for both prosthetic knee conditions. At 70\% preferred walking speed we found that vaulting of the intact ankle was significantly decreased while walking with the Rheo Knee II compared to the NMPK condition $(P=0.028)$. We did not find differences in peak vertical acceleration of the pelvis during initial and mid-swing of the prosthetic leg. In conclusion, comparison of walking with the Rheo Knee II to walking with a NMPK across different walking speeds showed limited differences in studied gait parameters. 


\section{INTRODUCTION}

Developments in prosthetic knee design have led to the introduction of microprocessorcontrolled prosthetic knees (MPKs), such as the Rheo Knee or C-Leg. MPKs should, in contrast to non-microprocessor-controlled prosthetic knees (NMPKs), allow early stance prosthetic knee flexion, ideal prosthetic knee kinematics during swing, and the ability to react to changes in walking speed. ${ }^{7,20}$

It is proposed that MPKs are beneficial for individuals with an amputation. Sawers and Hafner critically appraised the existing literature focusing on this proposition. ${ }^{22}$ They found four trials ${ }^{11,12,25,26}$ reporting an increase in preferred walking speed while using the MPK compared to a NMPK. They also found that comparison of other spatiotemporal variables were either inconsistent or not significant. Finally they found that the comparison of kinematic variables of walking with MPKs and NMPKs show "mixed and conflicting results".

The above-presented findings show no decisive evidence for an added value of MPKs on gait mechanics. One of the factors contributing to this might be that the majority of studies compared MPKs and NMPKs at preferred walking speed. As MPKs should be able to adapt to different walking speeds and NMPKs have restricted adaptive damping possibilities, differences between MPKs and NMPKs could be more pronounced at lower and/or higher walking speeds. This could be of importance as community ambulation requires walking at different speeds.

The available literature comparing MPKs and NMPKs across different walking speeds found no differences on spatiotemporal variables. ${ }^{28,31,139}$ We are aware of one study comparing prosthetic knee kinematics of walking with the Rheo Knee with walking with a NMPK. They found that in two out of four subjects peak prosthetic knee flexion during swing while walking with the Rheo Knee remained around 70 degrees (set target of the Rheo Knee during these measurements) irrespective of walking speed. ${ }^{7}$ Contrastingly, knee flexion while walking with the NMPK increased with walking speed. ${ }^{7}$ In the other subjects this was not visible, as they did not reach 70 degrees of prosthetic knee flexion during swing. ${ }^{7}$

Having more optimal prosthetic knee kinematics during swing can be beneficial for individuals with an amputation. Having too little prosthetic knee flexion might lead to problems with prosthetic foot clearance which, in turn, might lead to an premature ankle plantar flexion of the intact leg during mid-stance (vaulting) to assist with prosthetic foot clearance. ${ }^{19}$ Having too much prosthetic knee flexion during swing might also be undesirable, as the prosthetic knee has to be extended at the beginning of the stance phase. A larger peak prosthetic knee flexion during swing means that a larger movement trajectory has to be completed. The mechanism by which the prosthetic knee is extended during swing is not well studied, but in children without an amputation velocity-related forces and muscle activity of predominantly the stance leg have been described. ${ }^{140}$ During early and mid-stance, the hip abductors and extensors of the stance leg move the pelvis center of mass upwards. ${ }^{140}$ This movement creates an external knee extension moment. ${ }^{140}$ During slow walking stance 
limb muscle activity has shown to be the main contributor to knee extension during swing, while at faster walking the velocity-related forces are dominant. ${ }^{141}$ Whether these mechanisms are seen in individuals with an amputation and whether they are influenced by a user-adaptive prosthetic knee is unknown.

The aim of this study is to compare walking with a NMPK to walking with the Rheo Knee II across different walking speeds. We hypothesized an increased preferred walking speed while walking with the Rheo Knee II. In addition, we hypothesized comparable peak prosthetic knee flexion during swing across all walking speeds while walking with the Rheo Knee II, while peak prosthetic knee flexion during swing would increase with walking speed in the NMPK condition. Finally, we hypothesized a reduced vaulting of the intact leg at lower walking speeds and reduced vertical pelvic acceleration during initial swing of the prosthetic leg while walking with the Rheo Knee II when compared to the use of a NMPK. To contribute to the existing body of knowledge, we also analyzed spatiotemporal and kinematic variables reported in existing literature.

\section{Methods}

\section{Subjects}

For this randomized cross-over trial we recruited persons with a transfemoral amputation or knee disarticulation from the Netherlands and Belgium. The inclusion criteria were: (1) at least one year post amputation, (2) functional level from K2 (limited outdoor) or higher, ${ }^{142}$ and (3) never previously fitted with a microprocessor-controlled knee. Exclusion criteria were: (1) other musculoskeletal problems influencing walking ability, (2) stump problems / poor socket fitting, (3) body weight $>125 \mathrm{Kg}$ (maximum specification weight for the Rheo Knee II), and (4) knee center-floor distance below $41 \mathrm{~cm}$.

The Ethical Research Committee Twente, Enschede, the Netherlands approved the study protocol (NL 30112.044.09). All subjects provided written informed consent before the start of the measurements.

\section{Prosthetic adjustments}

We randomly assigned the subjects to start measurements with their own non-microprocessor-controlled prosthetic knee or with the Rheo Knee II. In both prosthetic knee conditions, the low-profile Vari-Flex ${ }^{\circledR}$ with EVO ${ }^{\text {TM }}$ prosthetic foot (Össur) was provided. After eight weeks of acclimatization the first set of measurements was performed after which subjects crossed over to the other prosthetic condition. After another eight weeks, the second set of measurements was performed and subjects left the research study. Full details regarding the process of prosthetic adjustments have been published before. ${ }^{143}$ Participants did not undergo a gait training program while walking with the Rheo Knee II or their own NMPK to make the comparison as little affected by gait training factors as possible. 


\section{Protocol}

Data were collected using the CAREN system (Motek Forcelink BV, Amsterdam, the Netherlands) at the Center for Augmented Learning and Training of the National Military Rehabilitation Center Aardenburg in Doorn, the Netherlands. This system consists of an instrumented single-belt treadmill and a twelve infrared-camera Vicon motion capture system (Oxford Metrics Ltd., Oxford, UK).

We used the modified Helen-Hayes marker set, including 37 reflective markers, which were placed according to the Vicon full-body Plug-in-Gait model. In addition, we placed two markers on the rope connecting the safety harness to an overhead frame. During a preliminary trial, we asked subjects to place their full bodyweight on the safety harness. The distance between the two markers in this condition was used in the data-analysis to check if subjects made use of the safety harness. The sample rate of the Vicon system was set at 100 samples per second.

Trials were performed at preferred walking speed, preferred walking speed $+15 \%$, and preferred walking speed $-30 \%$. The treadmill speed was fixed. We determined preferred walking speed during a familiarization trial.

The collected data were processed using Vicon Nexus 1.8 (Oxford Metrics Ltd., Oxford, UK). Initial contacts and toe-off were determined visually. Initial contact was defined as the moment the foot segment started to make a movement towards plantar flexion. The moment of toe-off was defined as the moment both the heel and toe marker trajectories changed from a backward to a forward movement. We loaded the processed data into customized Matlab 2010b software (The MathWorks, Inc, Natick, USA) for further analysis. We filtered the kinematic data using a zero-phase shift $2^{\text {nd }}$ order Butterworth filter with a cut-off frequency of $10 \mathrm{~Hz}$, meaning that the data were filtered twice with a $2^{\text {nd }}$ order filter. We selected 15 representative strides from the kinematic data. We resampled the kinematic data of each stride to 101 data points (0-100\% of stride cycle) to allow averaging over strides.

\section{Outcome measures}

We determined peak prosthetic knee flexion during swing. In addition, we calculated the amount of vaulting during the mid-stance of the intact leg. To do so, we subtracted the vertical position of the ankle joint center during the static calibration trial from the vertical position of the ankle joint center at the midpoint of the stance phase. Finally, we calculated the peak vertical acceleration of the pelvis during initial swing and mid swing of both the prosthetic and intact leg. To do so, we differentiated the position of the pelvic joint center, provided by the Vicon nexus software, twice in time intervals of 0.1 seconds.

Besides these outcome measures we calculated spatiotemporal and kinematic variables that have been previously reported in comparable trials to contribute to the existing body of knowledge. These include step length, the duration of the different gait phases (i.e. first double limb support, single limb stance, second double support, swing phase), knee yielding, defined as the difference between maximal knee flexion during early stance and knee 
Table 4.1: Descriptive variables participants

\begin{tabular}{ll}
\hline Variables & Persons with an amputation $(\mathbf{n}=\mathbf{1 0})$ \\
\hline Age (years) & $55(23-67)$ \\
Sex (male/female) & $5 / 4$ \\
Height (cm) & $175(158-189)$ \\
Weight (Kg) & $72.1(54.6-98.4)$ \\
Time since amputation (years) & $34(1-41)$ \\
Reason for amputation & Trauma (6), Infection (2), Osteosarcome (1) \\
Functional level & K2 (1), K3 (5), K4 (3) \\
Stump length (cm) & $40(32-60)$ \\
Non-microprocessor-controlled prosthetic knees & $3 R 60(4), 3 R 80(1)$, Mauch SNS (1), Graph \\
& Lite (1), CaTech (1) \\
\hline
\end{tabular}

Age, height, weight, time since amputation, and stump length are presented as median (range).

Sex, reason for amputation, functional level, and non-microprocessor-controlled prosthetic knees are presented as counts

flexion at initial contact, and peak hip extension during stance.

\section{Data analysis}

Statistical analysis on the outcome variables were performed using IBM SPSS Statistics 22 software (IBM SPSS Statistics, Chicago, USA). All outcome variables were compared between conditions using the Mann-Whitney $U$ test. In the evaluation of the effect of walking speed, each data point was used in two comparisons. To reduce the probability of a type II error due to multiple testing, we used the modified Holms-Bonferroni correction in the data analysis focusing on the effect of walking speed. The alpha level for all statistical comparisons was set to 0.05 .

\section{RESULTS}

A total of 61 participants were contacted of which 52 were potentially eligible based on the in- and exclusion criteria. Of these, 12 participants consented in participating. Three participants dropped out before the first measurements: one was not able to satisfactorily adjust to the Rheo Knee II, one because of stump problems, and one was not able to walk on a treadmill. The remaining nine participants completed both measurements. Characteristics of included participants are displayed in Table 4.1.

\section{Preferred walking speed}

Results of preferred walking speed are presented in Table 4.2. There was no significant difference in preferred walking speed between the Rheo Knee II and the NMPK condition. 


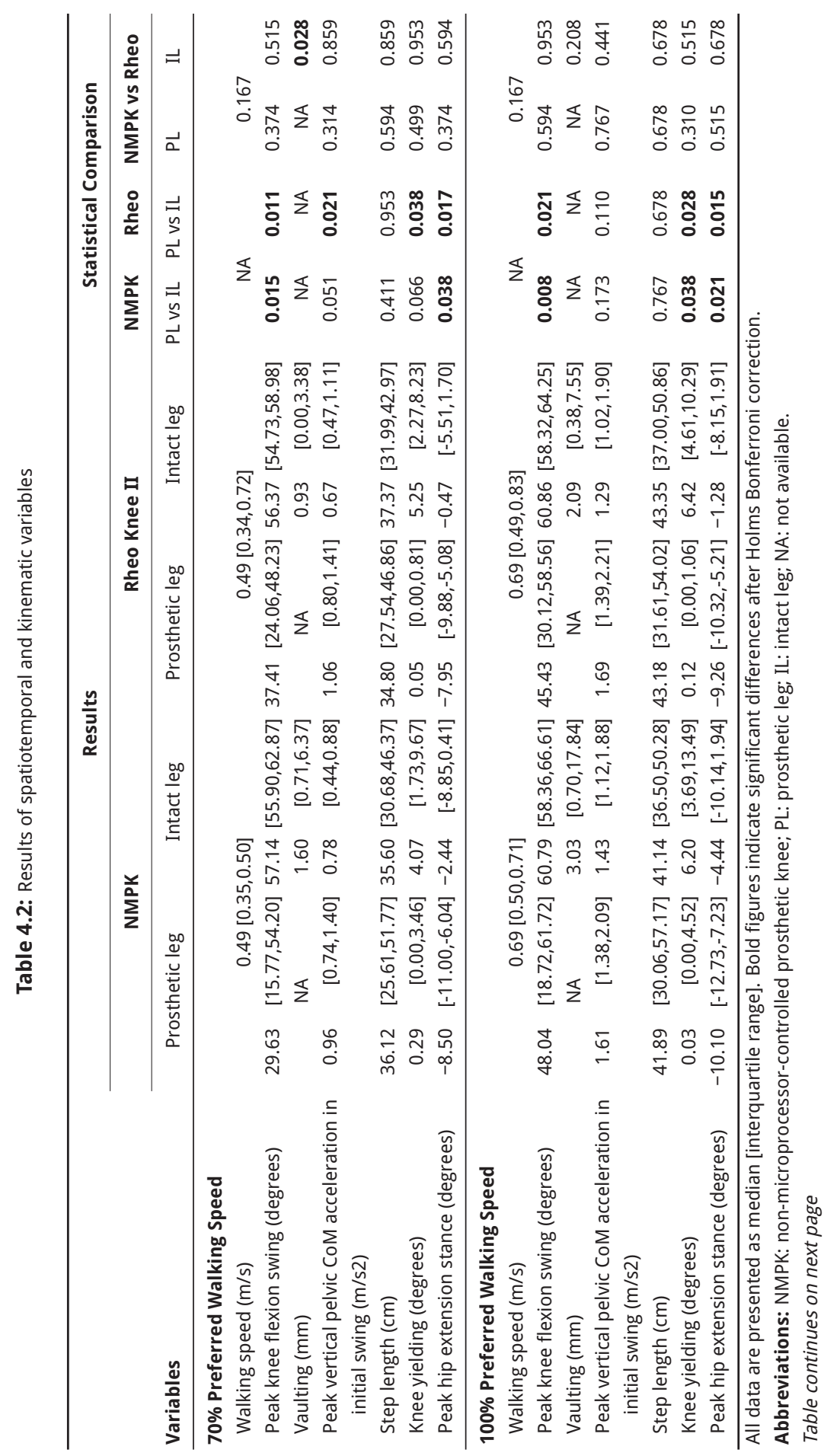




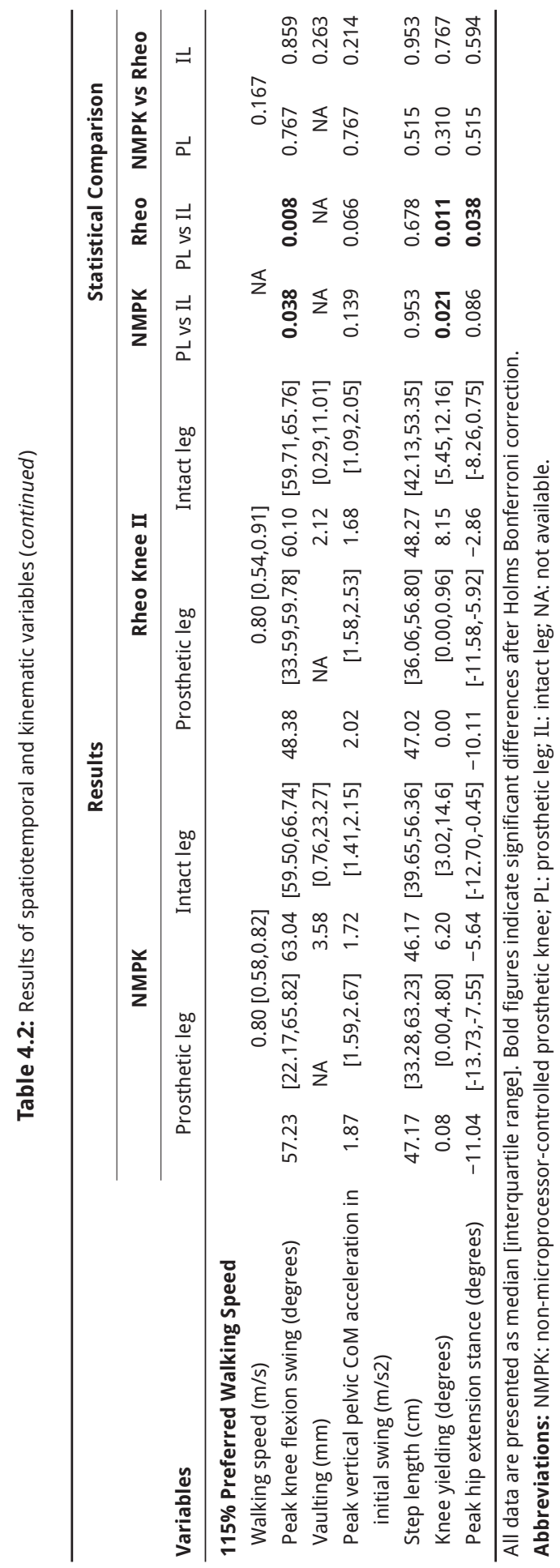




\section{Prosthetic knee flexion in swing}

The results of the comparison of the NMPK and Rheo Knee II condition within a walking speed condition are shown in Table 4.2, Figure 4.1, and Figure 4.2. The results of the comparison of the different walking speed conditions within a prosthetic knee condition are displayed in Table 4.3.

There were no statistically significant differences in peak prosthetic knee flexion during swing while walking with the Rheo Knee II and the NMPK within a walking speed condition. When looking at the influence of walking speed, we found that in both the Rheo Knee II and NMPK condition peak prosthetic knee flexion during swing increased significantly with walking speed.

\section{Vaulting and peak vertical acceleration of pelvic center of mass (CoM) in swing}

Results are presented in Table 4.2, Table 4.3, and Table 4.4.

We found that vaulting was significantly reduced while walking with the Rheo Knee II when compared to walking with the NMPK at 70\% preferred walking speed. In the other two walking speeds conditions no differences were found between both prosthetic knee conditions. Walking speed had no significant influence on the amount of vaulting in both prosthetic knee conditions.

The peak vertical acceleration of the pelvic CoM during initial and mid-swing of the prosthetic leg was not significantly different between the Rheo Knee II and the NMPK condition in any of the walking speed conditions. Comparison of the peak vertical pelvic CoM acceleration during the swing phase of the prosthetic and intact leg within a prosthetic knee showed one significant difference. In the Rheo Knee II condition a significantly higher peak vertical acceleration during the swing phase of the prosthetic leg at $70 \%$ preferred walking speed was found when compared to the swing phase of the intact leg. For the NMPK condition, the same comparison was on the borderline of significance. We found that for both the Rheo Knee II and the NMPK peak acceleration of the pelvic center of mass significantly increased with walking speed. This was seen for both the prosthetic and intact leg. The only exception to this was the comparison of the intact leg at preferred walking speed and $115 \%$ preferred walking speed in the Rheo Knee II condition.

\section{Previously reported outcome measures}

For the results of the comparisons on the other outcome variables, see Table 4.2, 4.3, and 4.4. On previously reported outcome parameters no differences were found between the Rheo Knee II and the NMPK condition across all walking speeds. Walking speed had a significant influence on almost all outcome parameters for both prosthetic knee conditions. 

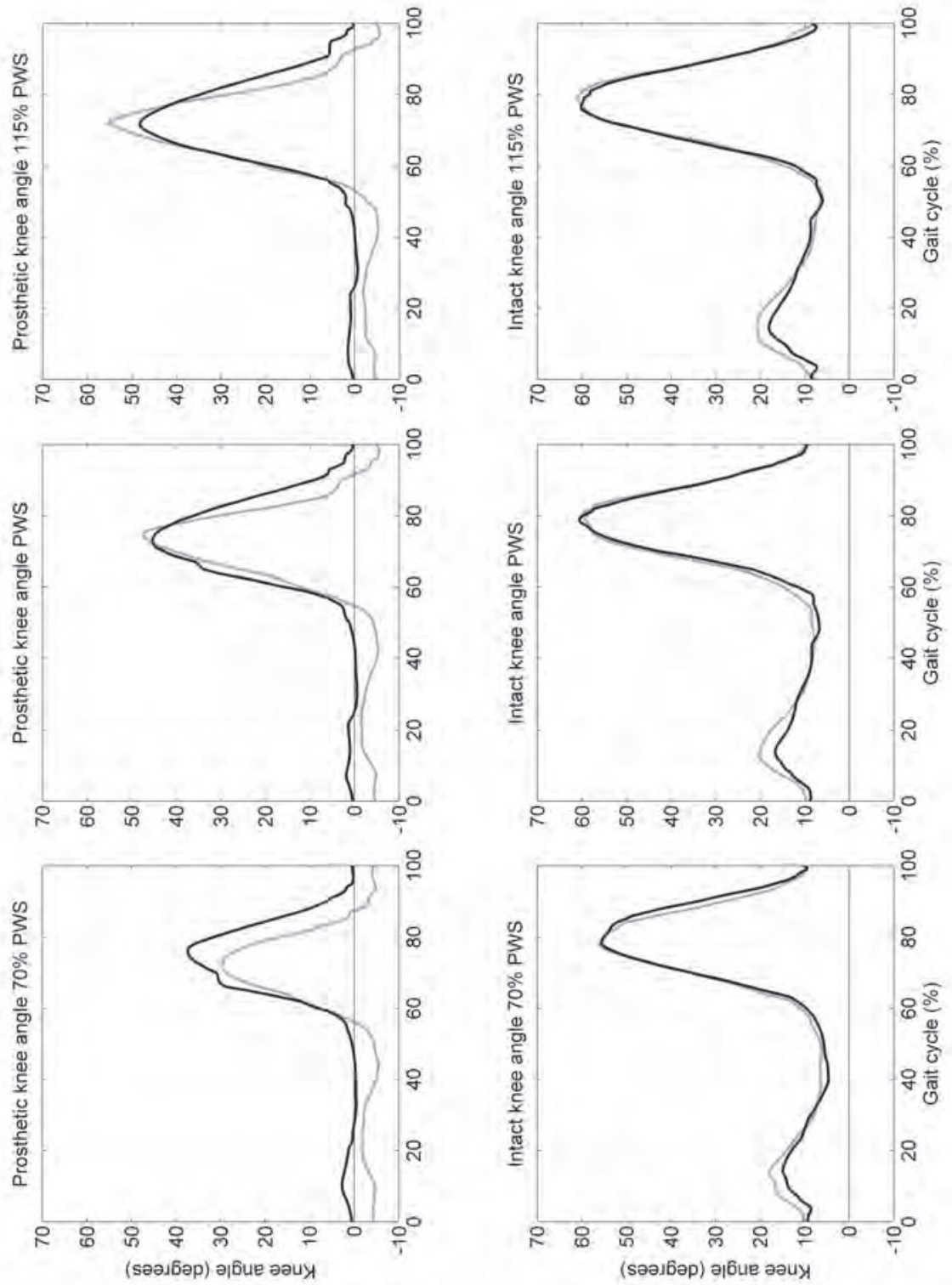

Figure 4.1: Influence of walking speed on knee kinematics.

This figure shows the median knee kinematics of both prosthetic knee conditions at the different walking speeds. The data of the Rheo Knee II condition are shown as a solid black line, the data of the non-microprocessorcontrolled prosthetic knee are shown as a solid grey line. Abbreviation: PWS: preferred walking speed. 


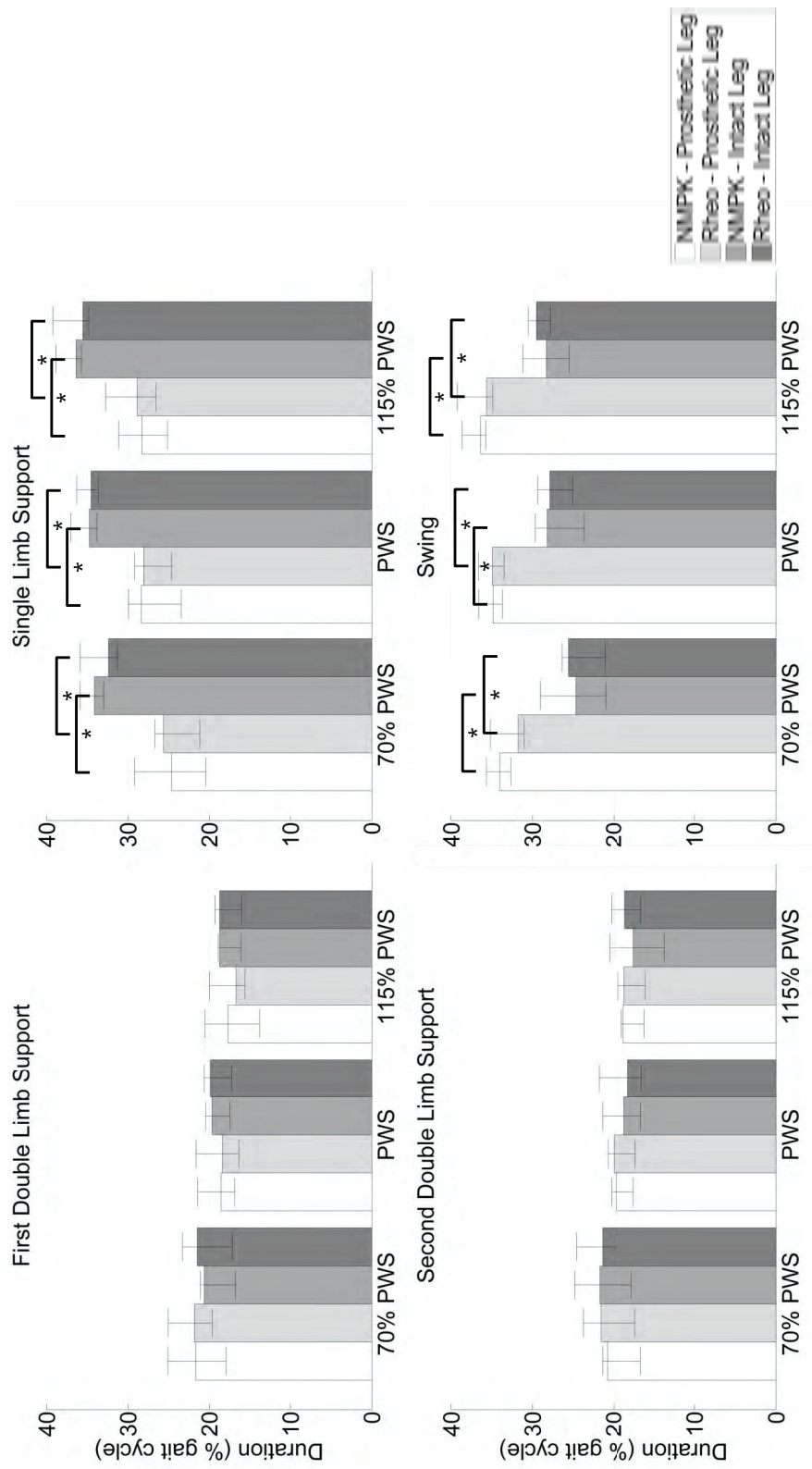

Figure 4.2: Graphical representation of the duration of the different gait phases.

The bars show the median values, the error bar depicts the interquartile range. * Denotes a statistical significant difference $(P=0.008)$. Abbreviation: PWS: preferred walking speed 
Table 4.3: Influence of walking speed on the prosthetic leg

\begin{tabular}{|c|c|c|c|c|c|c|c|c|}
\hline \multirow[b]{3}{*}{ Variables } & \multicolumn{8}{|c|}{ Non-microprocessor-controlled prosthetic knee } \\
\hline & \multicolumn{3}{|c|}{$70 \%$ PWS vs PWS } & \multicolumn{3}{|c|}{$70 \%$ PWS vs $115 \%$ PWS } & \multicolumn{2}{|c|}{ PWS vs $115 \%$ PWS } \\
\hline & Medic & n difference & $P$ & Media & n difference & $P$ & Median difference & $P$ \\
\hline Step length $(\mathrm{cm})$ & -0.06 & {$[-0.89,-0.01]$} & 0.021 & -0.10 & {$[-0.14,-0.08]$} & 0.024 & $-0.05[-0.07,-0.03]$ & 0.016 \\
\hline First DLS (\% GC) & 2.29 & {$[1.45,4.03]$} & 0.028 & 4.07 & {$[3.26,5.11]$} & 0.016 & $1.65[0.52,2.33]$ & 0.024 \\
\hline SLS (\% GC) & -2.20 & {$[-3.16,-1.13]$} & 0.024 & -3.32 & {$[-4.25,-2.10]$} & 0.016 & $-0.86[-1.63,-0.31]$ & 0.021 \\
\hline Second DSP (\% GC) & 0.71 & {$[-0.76,0.99]$} & 0.441 & 2.07 & {$[0.86,2.17]$} & 0.022 & $1.30 \quad[0.80,1.69]$ & 0.024 \\
\hline Swing (\% GC) & -1.58 & {$[-2.41,0.77]$} & 0.260 & -3.30 & {$[-3.45,-2.18]$} & 0.008 & $-1.73[-2.84,-1.13]$ & 0.016 \\
\hline $\begin{array}{l}\text { Peak vertical pelvic CoM } \\
\text { acc. initial swing (m/s2) }\end{array}$ & -0.65 & {$[-0.80,-0.47]$} & 0.024 & -0.95 & {$[-1.35,-0.68]$} & 0.016 & $-0.26[-0.44,-0.07]$ & 0.011 \\
\hline Knee Yielding (degrees) & 0.00 & {$[-0.30,0.08]$} & 1.000 & 0.00 & {$[-0.63,0.11]$} & 0.686 & $0.00 \quad[-0.08,0.01]$ & 1.000 \\
\hline $\begin{array}{l}\text { Peak knee flexion swing } \\
\text { (degrees) }\end{array}$ & -4.94 & {$[-12.48,-2.95]$} & 0.024 & -10.78 & {$[-18.91,-3.55]$} & 0.022 & $-5.34[-7.52,-0.60]$ & 0.011 \\
\hline \multirow[t]{4}{*}{$\begin{array}{l}\text { Peak hip extension stance } \\
\text { (degrees) }\end{array}$} & 1.26 & {$[0.32,2.18]$} & 0.030 & 1.58 & {$[0.81,3.21]$} & 0.033 & $0.70 \quad[-0.12,1.35]$ & 0.048 \\
\hline & \multicolumn{8}{|c|}{ Rheo Knee II } \\
\hline & \multicolumn{3}{|c|}{$70 \%$ PWS vs PWS } & \multicolumn{3}{|c|}{$70 \%$ PWS vs $115 \%$ PWS } & \multicolumn{2}{|c|}{ PWS vs $115 \%$ PWS } \\
\hline & Medic & n difference & $P$ & Media & n difference & $P$ & Median difference & $P$ \\
\hline Step length (cm) & -0.05 & {$[-0.08,-0.04]$} & 0.008 & -0.08 & {$[-0.13,-0.06]$} & 0.016 & $-0.03[-0.05,-0.03]$ & 0.024 \\
\hline First DLS (\% GC) & 3.64 & {$[1.68,5.28]$} & 0.024 & 5.35 & {$[3.65,6.54]$} & 0.016 & $1.68 \quad[1.03,2.01]$ & 0.008 \\
\hline SLS (\% GC) & -3.15 & {$[-3.89,-2.07]$} & 0.024 & -4.44 & {$[-5.30,-3.47]$} & 0.016 & $-1.35[-1.83,-0.77]$ & 0.008 \\
\hline Second DSP (\% GC) & 1.36 & {$[0.04,3.02]$} & 0.028 & 2.66 & {$[1.53,3.56]$} & 0.016 & $1.29[0.55,2.27]$ & 0.024 \\
\hline Swing (\% GC) & -1.43 & {$[-3.46,-0.65]$} & 0.016 & -3.91 & {$[-4.73,-2.30]$} & 0.024 & $-1.65[-2.60,-0.83]$ & 0.008 \\
\hline $\begin{array}{l}\text { Peak vertical pelvic CoM } \\
\text { acc. initial swing (m/s2) }\end{array}$ & -0.54 & {$[-0.80,-0.40]$} & 0.016 & -1.01 & {$[-1.27,-0.38]$} & 0.024 & $-0.29 \quad[-0.62,0.01]$ & 0.038 \\
\hline Knee Yielding (degrees) & 0.00 & {$[-0.47,0.35]$} & 0.753 & 0.00 & {$[-0.59,0.40]$} & 1.000 & $0.00 \quad[-0.26,0.06]$ & 1.000 \\
\hline $\begin{array}{l}\text { Peak knee flexion swing } \\
\text { (degrees) }\end{array}$ & -5.43 & {$[-10.33,-1.05]$} & 0.021 & -8.85 & {$[-11.58,-3.42]$} & 0.022 & $-2.72[-3.47,-1.02]$ & 0.024 \\
\hline $\begin{array}{l}\text { Peak hip extension stance } \\
\text { (degrees) }\end{array}$ & 0.45 & {$[-0.58,3.06]$} & 0.260 & 1.10 & {$[0.61,3.71]$} & 0.024 & $1.09[0.63,1.37]$ & 0.022 \\
\hline
\end{tabular}

All data are presented as median [interquartile range].

Abbreviations: PWS: preferred walking speed; DLS: double limb support; GC: gait cycle; SLS: single limb support; CoM: center of mass; acc: acceleration. 
Table 4.4: Influence of walking speed on the intact leg

\begin{tabular}{|c|c|c|c|c|c|c|c|}
\hline \multirow[b]{3}{*}{ Variables } & \multicolumn{7}{|c|}{ Non-microprocessor-controlled prosthetic knee } \\
\hline & \multicolumn{2}{|c|}{$70 \%$ PWS vs PWS } & \multicolumn{3}{|c|}{$70 \%$ PWS vs $115 \%$ PWS } & \multicolumn{2}{|c|}{ PWS vs $115 \%$ PWS } \\
\hline & Median difference & $P$ & Medic & n difference & $P$ & Median difference & $P$ \\
\hline Step length (cm) & $-0.05[-0.09,-0.04]$ & 0.016 & -0.09 & {$[-0.12,-0.16]$} & 0.024 & $-0.04[-0.06,-0.03]$ & 0.011 \\
\hline First DLS (\% GC) & $0.29 \quad[-0.70,1.29]$ & 0.441 & 1.88 & {$[0.98,2.43]$} & 0.022 & $1.41[0.83,1.68]$ & 0.016 \\
\hline SLS (\% GC) & $-1.03[-1.96,-0.09]$ & 0.066 & -2.78 & {$[-3.19,-2.29]$} & 0.016 & $-1.76[-2.40,-1.19]$ & 0.024 \\
\hline Second DSP (\% GC) & {$[1.92,3.52]$} & 0.011 & 4.36 & {$[3.49,4.78]$} & 0.024 & $1.54 \quad[0.52,2.37]$ & 0.016 \\
\hline Swing (\% GC) & $-2.20[-2.85,-1.24]$ & 0.024 & -3.27 & {$[-3.86,-2.25]$} & 0.016 & $-0.96 \quad[-1.77,0.38]$ & 0.015 \\
\hline Vaulting (mm) & $-1.50[-11.47,0.00]$ & 0.086 & -2.05 & {$[-14.95,-0.11]$} & 0.075 & $-0.41 \quad[-2.12,0.15]$ & 0.093 \\
\hline $\begin{array}{l}\text { Peak vertical pelvic CoM } \\
\text { acc. initial swing (m/s2) }\end{array}$ & $-0.62[-0.91,-0.51]$ & 0.008 & -1.00 & {$[-1.12,-0.85]$} & 0.024 & $-0.30[-0.47,-0.19]$ & 0.008 \\
\hline Knee Yielding (degrees) & $-2.13[-4.33,-0.37]$ & 0.045 & -2.13 & {$[-5.57,-0.82]$} & 0.102 & $-0.93 \quad[-1.41,0.00]$ & 0.345 \\
\hline $\begin{array}{l}\text { Peak knee flexion swing } \\
\text { (degrees) }\end{array}$ & $-4.15 \quad[-5.67,-1.06]$ & 0.024 & -4.54 & {$[-7.06,-2.64]$} & 0.022 & $-0.64[-1.82,-0.15]$ & 0.028 \\
\hline \multirow[t]{4}{*}{$\begin{array}{l}\text { Peak hip extension stance } \\
\text { (degrees) }\end{array}$} & $0.34 \quad[-1.10,2.33]$ & 0.314 & 3.19 & {$[0.50,3.87]$} & 0.042 & {$[0.46,2.99]$} & 0.033 \\
\hline & \multicolumn{7}{|c|}{ Rheo Knee II } \\
\hline & \multicolumn{2}{|c|}{$70 \%$ PWS vs PWS } & \multicolumn{3}{|c|}{$70 \%$ PWS vs $115 \%$ PWS } & \multicolumn{2}{|c|}{ PWS vs $115 \%$ PWS } \\
\hline & Median difference & $P$ & Medic & n difference & $P$ & Median difference & $P$ \\
\hline Step length (cm) & $-0.05 \quad[-0.08,-0.04]$ & 0.016 & -0.10 & {$[-0.11,-0.08]$} & 0.024 & $-0.05[-0.05,-0.02]$ & 0.008 \\
\hline First DLS (\% GC) & $1.40 \quad[-0.10,2.51]$ & 0.051 & 2.75 & {$[1.35,3.30]$} & 0.024 & $1.16[0.59,2.08]$ & 0.016 \\
\hline SLS (\% GC) & $-1.26 \quad[-2.72,0.20]$ & 0.051 & -3.47 & {$[-4.00,-2.11]$} & 0.016 & $-1.73[-2.58,-1.04]$ & 0.024 \\
\hline Second DSP (\% GC) & $3.31 \quad[1.64,4.46]$ & 0.024 & 4.81 & {$[3.76,6.07]$} & 0.008 & $1.93[1.48,2.44]$ & 0.016 \\
\hline Swing (\% GC) & $-3.35[-10.55,3.17]$ & 0.260 & -4.62 & {$[-5.61,-3.38]$} & 0.024 & $0.50 \quad[-3.89,2.03]$ & 0.678 \\
\hline Vaulting (mm) & $0.27 \quad[-1.95,1.76]$ & 0.086 & -0.59 & {$[-7.86,-0.10]$} & 0.054 & $-0.21 \quad[-2.56,0.00]$ & 0.063 \\
\hline $\begin{array}{l}\text { Peak vertical pelvic CoM } \\
\text { acc. initial swing (m/s2) }\end{array}$ & $-0.68 \quad[-0.78,-0.48]$ & 0.016 & -0.89 & {$[-1.09,-0.61]$} & 0.024 & $-0.29 \quad[-0.41,0.06]$ & 0.086 \\
\hline Knee Yielding (degrees) & $-2.24[-3.83,-0.52]$ & 0.022 & -3.06 & {$[-4.86,-0.81]$} & 0.084 & $-1.18 \quad[-1.80,0.12]$ & 0.278 \\
\hline $\begin{array}{l}\text { Peak knee flexion swing } \\
\text { (degrees) }\end{array}$ & $-4.75 \quad[-5.62,-1.70]$ & 0.024 & -3.96 & {$[-6.91,-2.28]$} & 0.016 & $-0.91 \quad[-2.33,0.48]$ & 0.314 \\
\hline $\begin{array}{l}\text { Peak hip extension stance } \\
\text { (degrees) }\end{array}$ & $0.79 \quad[-0.57,2.53]$ & 0.173 & 2.48 & {$[1.62,3.19]$} & 0.033 & {$[0.36,1.94]$} & 0.022 \\
\hline
\end{tabular}

All data are presented as median [interquartile range].

Abbreviations: PWS: preferred walking speed; DLS: double limb support; GC: gait cycle; SLS: single limb support; CoM: center of mass; acc: acceleration. 


\section{Discussion}

The aim of this study was to compare the use of a NMPK to the use of the Rheo Knee II across different walking speeds. The comparison of the NMPK and Rheo Knee II showed limited differences in gait parameters across all three evaluated walking speeds.

We hypothesized that patients would adopt a higher preferred walking speed while walking with Rheo Knee II when compared to the NMPK. Our results disproved this hypothesis: we did not find a difference in preferred walking speed between the Rheo Knee II and NMPK condition. Differences in preferred walking speed between user-adaptive prosthetic knees and NMPKs have both been found to be significant (increased in user-adaptive knees) ${ }^{12,25,26,144}$ and non-significant. ${ }^{11,139}$ The reason for this ambiguity in results is unclear, but possible explanations include difference in study population, duration of acclimatization period and the presence/absence of a training protocol to get used to the user-adaptive prosthetic knee.

We further hypothesized that peak prosthetic knee flexion during swing while walking with the Rheo Knee II would be comparable in the three walking speed conditions, while prosthetic knee flexion would increase with walking speed while walking with the NMPK. Although this pattern was visible in our results (see Figure 4.1), these differences were not statistically significant within each of the walking speed conditions. In addition, when comparing peak prosthetic knee flexion during swing across walking speeds (see Table 4.3), we observed that prosthetic knee flexion significantly increased with walking speed in both prosthetic knee conditions. We were, thus, not able to confirm our hypothesis in our study population. One of the contributing factors might be that five subjects had a relatively low peak prosthetic knee flexion (less than 45 degrees at preferred walking speed) during swing while walking with the NMPK and three had a relatively high peak prosthetic knee flexion in swing (more than 65 degrees at preferred walking speed). The subjects with a low peak prosthetic knee flexion increased their peak prosthetic knee flexion during swing while walking with the Rheo Knee II, and the subjects with a high peak prosthetic knee flexion decreased it. In the non-parametric statistical analysis, this lead to both positive and negative ranks leading to non-significant differences. The same pattern was visible at the faster and slower walking speeds.

We also looked at the compensatory movements of the intact leg, that might be associated with non-optimal prosthetic knee kinematics such as intact ankle vaulting and the peak vertical acceleration of the pelvis. We found that at $70 \%$ preferred walking speed, vaulting while walking with the Rheo Knee II was significantly decreased when compared to walking with the NMPK. This could be due to the fact that peak prosthetic knee flexion during swing was lower in the NMPK condition when compared to the Rheo Knee II condition (median difference [IQR]: -7.86 [-17.26, 2.83]). This difference, however, was not significant. The reduced peak knee flexion during swing while walking with the NMPK might have led to foot clearance problems, which may have increased vaulting of the intact leg to prevent toe drag 
of the prosthetic leg. We also looked at the peak vertical acceleration of the pelvic CoM during the swing phase of the prosthetic leg and found no differences between prosthetic knees across walking speeds. We chose to look into this variable, because research in typically developing children the upward movement of the pelvis and velocity-related forces are the main contributors to the knee extension movement during swing. ${ }^{140}$ However, we do not know if and how these findings are applicable on individuals with an amputation. This means that these results are exploratory of nature and should be interpreted with caution. Future research should elucidate the mechanism by which the prosthetic knee is extended during swing and if, and to what extent, a user-adaptive prosthetic knee could influence this mechanism.

Finally, we studied outcome parameters that have been reported in existing literature. We found no significant differences in these outcome parameters between prosthetic knees. Figure 4.1 suggests that prosthetic knee flexion at initial contact was higher while walking with the Rheo Knee II when compared to the NMPK condition. We, however, believe that this is due to differences in alignment between prosthetic knees. As previously conducted trials show mixed and conflicting results on spatiotemporal and kinematic variables, our results are both in line and conflicting with previously conducted trials. If we look at knee yielding for instance, both trials reporting no differences in knee yielding ${ }^{11,12,144}$ as an increase in knee yielding ${ }^{7,29}$ are available (respectively 8 and 1 degrees).

We think that a number of confounding factors may have contributed to the limited differences we found. At first, we had a small sample size, which affected statistical power and thereby the ability to detect significant differences. However, if marked differences between prosthetic knee conditions existed, these differences might have been identified. In addition, we provided eight weeks of acclimatization to the Rheo Knee II. This may have been too short for full acclimatization which might have affected the outcome of the studied variables. Thirdly, we chose to leave out a gait training because it is not common in the Netherlands to receive a gait training program after prescription of a microprocessorcontrolled prosthetic knee. In addition, previous research has shown that gait re-education can influence the studied outcome parameters, even without altering the prosthetic components. ${ }^{71}$ This means that in a research setting a gait training program always should be given to both prosthetic knee conditions which was practically not feasible due to the required time investment.

In conclusion, we found that walking with the Rheo Knee II lead to a reduced vaulting of the intact leg when compared to the NMPK condition at $70 \%$ preferred walking speed. On other gait parameters no differences were found. 


\section{Added value of a user-adaptive prosthetic knee in planned gait initiation: Off to a good start?}

Prinsen EC, Nederhand MJ, Rietman JS, Koopman HFJM. IEEE Int Conf Rehabil Robot 2015;Aug:31-36. 


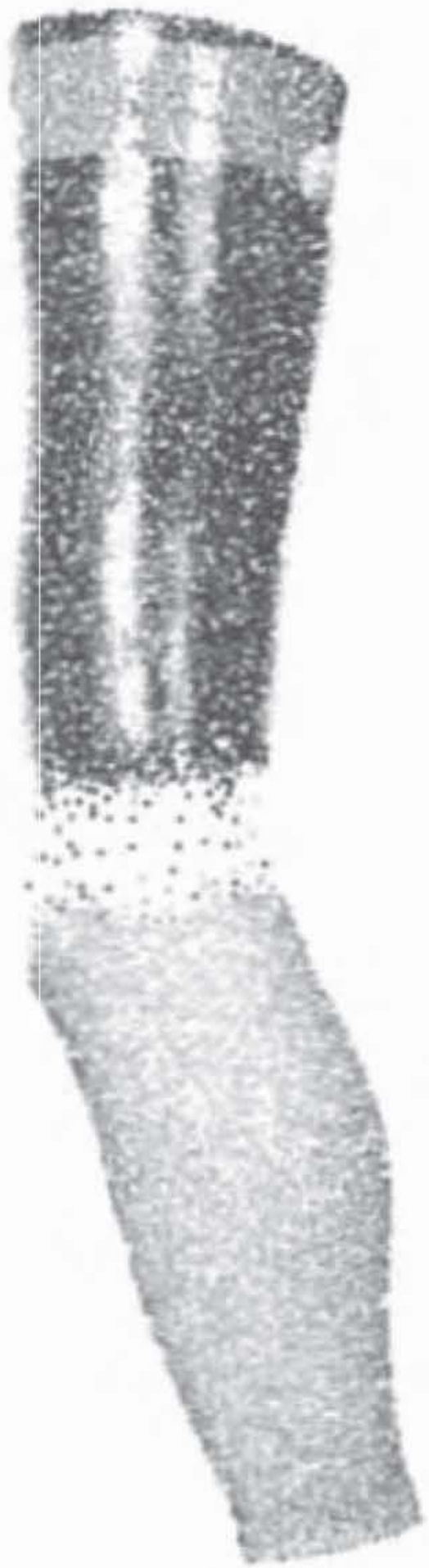




\section{ABstract}

Advancements in prosthetic knee design have led to biologically inspired microprocessorcontrolled prosthetic knees. The added value of these devices has been extensively studied in walking. However, trials into the activity pattern of persons with an amputation show that the majority of ambulation activities are short in duration. This means that a high percentage of ambulatory activities involves the initiation of gait. Therefore the aim of this paper is to study the added value of a user-adaptive prosthetic knee in gait initiation. Ten persons with an amputation were included and measured with their own non-microprocessorcontrolled prosthetic knee and with the Rheo Knee II. In addition, a control group of ten persons without an amputation were included. Measurements were performed using two force plates and a six-camera Vicon system. The persons with an amputation started gait alternately with their intact and prosthetic leg. The results indicated that persons with an amputation heavily rely on the intact leg for the generation of propulsive forces. In addition, the reduced role of the prosthetic leg led to a lower velocity of the centre of mass at the end of the first step in persons with an amputation when compared to the control subjects. The transition towards the Rheo Knee II had no effect on the studied outcome parameters. In conclusion, there is no effect of the transition towards a user-adaptive prosthetic knee on intact leg reliance seen during gait initiation. 


\section{INTRODUCTION}

Advancements of prosthetic knee design have led to the introduction of microprocessorcontrolled adaptive prosthetic knees, such as the C-Leg and Rheo Knee II. Conventional nonadaptive prosthetic knees are limited in adapting damping properties. In contrast, adaptive prosthetic knees are capable of adapting the damping properties of the knee to, for instance, walking speed. These prosthetic knees should allow early stance knee flexion, ideal knee kinematics during swing and improved stance stability. ${ }^{7,20}$ The added value of adaptive prosthetic knees has been studied on a number of different tasks, but the majority of these trials studied level walking. However, a study into the activity pattern of persons with an amputation showed that the majority of activity bouts were 1 or 2 minutes in length. ${ }^{145}$ This result indicates that a high percentage of ambulatory activity of persons with an amputation involves initiating gait. However, the added value of a microprocessor-controlled adaptive prosthetic knee during gait initiation has not been studied yet.

Previous research studying gait initiation in participants with an amputation has shown reduced involvement of the prosthetic leg in the generation of the required propulsive forces. ${ }^{146-151}$ There are multiple explanations for this finding. First of all, the majority of prosthetic ankles lack active ankle function, which is needed for the posterior translation of the centre of pressure (CoP). ${ }^{146,152}$ This impairs the mechanism by which propulsive forces are usually generated during gait initiation: through a posterior shift of the CoP the centre of mass (CoM) starts moving forwards due to the force of gravity. ${ }^{153}$ In addition, earlier conducted trials showed a reduced duration of the single prosthetic limb support in the leading intact leg condition. ${ }^{146-149}$ Single limb support on the prosthetic leg is usually reduced as much as possible because of limitations in balance control of the prosthetic leg. ${ }^{11,31}$ Reduction in the duration of the single limb support means that a limited amount of propulsive forces can be generated by the prosthetic leg. Finally, previous studies have shown decreased decoupling of the CoM and CoP when gait is initiated with the prosthetic leg. ${ }^{146-149}$ This could be explained by the fact that in this situation the leading prosthetic leg must absorb the forces that are associated the CoM acceleration during the first step. Nonmicroprocessor-controlled prosthetic knee have limitations in allowing early stance knee flexion. A large decoupling of the COM and CoP in the leading prosthetic leg condition would increase the risk on knee buckling and consequently falls when walking with a nonmicroprocessor-controlled prosthetic knee.

Because microprocessor-controlled adaptive prosthetic knees should increase stance stability and allow early stance flexion, they could be of added value during gait initiation. However, this has not been studied before. Therefore we aim to investigate the added value of the Rheo Knee II on gait initiation. We hypothesized an increased decoupling of the CoM and COP by increasing the duration of the EXE phase in the leading intact leg. In addition, we hypothesized a larger decoupling of the CoM and CoP in the leading prosthetic leg condition by increased stance stability and early stance flexion of the prosthetic knee. 


\section{MethOdS}

\section{Subjects}

For this study, participants with a transfemoral amputation were recruited from the Netherlands and Belgium. Inclusion criteria were: (1) at least one year post amputation, (2) functional level ranging from K2 (limited outdoor) to K4 (active athlete), ${ }^{142}$ and (3) never supplied with a microprocessor-controlled prosthetic knee before. Exclusion criteria were: (1) other musculoskeletal problems influencing walking ability, (2) stump problems or bad socket fitting, (3) body weight exceeding $125 \mathrm{Kg}$ (maximal tolerable weight of the Rheo Knee II), and (4) knee center-floor distance below $41 \mathrm{~cm}$ (minimum height needed to fit the Rheo Knee II). In addition, ten control subjects without an amputation were recruited. Controls were eligible when they were free of musculoskeletal problems influencing walking ability. The study protocol was approved by the Medical Ethical Research Committee Twente, Enschede, the Netherlands and all subjects provided written informed consent.

\section{Prosthetic adjustments}

The persons with an amputation were randomly assigned to start the measurements with their own non-microprocessor-controlled prosthetic knee or to start the measurements with the Rheo Knee II. In both prosthetic knee conditions, the low-profile Vari-Flex ${ }^{\circledR}$ with $\mathrm{EVO}^{\mathrm{TM}}$ pros-thetic foot (Össur) was provided. After eight weeks of acclimatization the first set of measurements was performed after which the subjects crossed over to the other prosthetic knee condition. After another eight weeks, the second set of measurements was performed and subjects left the study. Participants did not undergo a gait training program while walking with the Rheo Knee II. Our main consideration in leaving out a gait training program was that we aimed to make the comparison as little affected by gait training factors as possible.

\section{Measurement protocol}

Kinematic and force plate data were collected using a six-camera Vicon motion capture system (Oxford Metrics Ltd., Oxford, UK) and two AMTI force plates (Advanced Mechanical Technology Incorporated, Watertown, MA, USA). Thirty-five reflective markers were placed on anatomical landmarks according to the Vicon full-body Plug-in-Gait model. The marker trajectories were recorded at a frequency of $100 \mathrm{~Hz}$. Force plate data were recorded with a frequency of $1000 \mathrm{~Hz}$.

For the measurements, participants stood with a foot on a force plate each while looking straight ahead. Foot placement was standardized using a mold. After an auditory signal was given, participants started to walk. The persons with an amputation started alternately with their prosthetic and intact leg. Control subjects could start trials with their preferred leg, which was noted. The average scores of five trials were used in the analysis.

The collected data were processed using Vicon Nexus 1.8 and loaded into customized 
Matlab software (The MathWorks Inc, Natick, USA) for further analysis. We filtered data using a zero-phase shift $2^{\text {nd }}$ order Butterworth filter with a cut-off frequency of $10 \mathrm{~Hz}$.

\section{Outcome measures}

One of the most used outcome variables in previous trials studying gait initiation is the horizontal decoupling of the CoM and CoP. However, this variable has limitations because it does not take the CoM velocity into account. ${ }^{154}$ To incorporate CoM velocity, Hof et al. ${ }^{155}$ introduced a new variable: the extrapolated CoM (XcoM). The XcoM is calculated by adding the ratio of the CoM velocity and the eigen-frequency of the inverted pendulum to the CoM position. See equation (5.1) in which XcoM is the XcoM position, CoM is the CoM position, $g$ is the gravitational constant, and I is the pendulum length.

$$
X \operatorname{coM}=C o M+\frac{C \dot{\circ} M}{\omega_{n}}, \omega_{n}=\sqrt{\frac{g}{l}}
$$

Experiments showed that the maximal mediolateral distance between the XcoM and CoP, while still maintaining balance, was smaller in amplitude when compared to maximal distance between CoM and CoP. ${ }^{155}$ These result indicate that the XcoM is a more conservative variable to quantify mediolateral stability. We decided to calculate the horizontal decoupling (in anteroposterior direction) of the XcoM and the CoP next to the generally accepted method of calculating horizontal decoupling of the CoM and CoP. We calculated the following outcome measures: (1) duration of the anticipatory postural adjustment (APA) phase in which the required propulsive forces are generated needed to lift the leading leg of the ground. The duration is defined as the time between the moment the anteroposterior ground reaction force exceeded $1 \%$ of the body weight and toe-off of the leading leg; ${ }^{149}$ (2) duration of the step execution phase (EXE), defined as the time between toe-off and initial contact of the leading leg; (3) maximal decoupling of the CoM/XcoM and CoP during the APA phase for the leading and trailing leg; (4) maximal decoupling of the CoM/XcoM and COP during the EXE phase for the trailing leg; (5) anteroposterior impulse generated by the leading and intact leg during the APA phase, calculated by trapezoidal integration of the anteroposterior component of the ground reaction force; (6) anteroposterior impulse generated by the trailing leg during the EXE phase; (7) CoM velocity at the end of the APA and EXE phase, calculated by numerical differentiation of the CoM trajectory in time intervals of $0.1 \mathrm{sec}$; (8) step length first step; (9) knee angle at initial contact of the leading leg; and (10) knee yielding defined as the difference between maximal knee flexion during early stance and the knee flexion angle at initial contact.

\section{Statistical analysis}

Data were analyzed using IBM SPSS statistics 22 software (IBM SPSS Statistics, Chicago, USA). We applied non-parametric statistics using the Wilcoxon Signed Rank Sum Test to compare the data of the people with an amputation. We used the Mann-Whitney $U$ test 
Table 5.1: Descriptive variables participants

\begin{tabular}{lll}
\hline Variables & $\begin{array}{l}\text { Persons with an amputation } \\
(\mathbf{n}=\mathbf{1 0})\end{array}$ & $\begin{array}{l}\text { Control subjects } \\
\text { (n= 10) }\end{array}$ \\
\hline Age (years) & $53(23-67)$ & $28(26-56)$ \\
Sex (male/female) & $6 / 4$ & $5 / 5$ \\
Time since amputation (years) & $33.5(1-41)$ & Not applicable \\
Reason for amputation & Trauma (7), Infection (2), & Not applicable \\
& Osteosarcome (1) & \\
Functional level & K2 (2), K3 (5), K4 (3) & Not applicable \\
Stump length (cm) & 40 (32 - 60) & Not applicable \\
Non-microprocessor-controlled & 3R60 (4), 3R80 (1), Mauch SNS (1), & Not applicable \\
prosthetic knees & Graph Lite (1), CaTech (1), Total & \\
& Knee 2000 (1) & \\
\hline
\end{tabular}

Age, time since amputation, and stump length are presented as median (range). Sex, reason for amputation, functional level, and non-microprocessor-controlled prosthetic knees are presented as counts

to compare the data of the intact leg with the control subjects. Because each data point is used in multiple comparisons, we used the modified Holms-Bonferroni method to correct for multiple testing. The $p$-values for the comparisons in which a data point was used were ranked from small to large. As the data are used in 3 comparisons, the smallest $p$ value should be $\leq 0.017$ ( $\alpha$ of 0.05 divided by 3 ), the middle $p$-value should be $\leq 0.025$ ( $\alpha$ of 0.05 divided by 2 ), and the largest $p$-value should be $\leq 0.05$ ( $\alpha$ of 0.05 divided by 1 ).

\section{RESULTS}

\section{Subject recruitment}

A group of twelve persons with an amputation consented to participate in this study. Two participants dropped out before the first measurements were completed. One was not able to satisfactorily adjust to the Rheo Knee II and one subject dropped out because of stump problems. The remaining ten participants completed both measurements. In addition, a control group of ten persons without an amputation was included and measured. Characteristics of participants are displayed in Table 5.1.

\section{Comparison Leading Intact and Leading Prosthetic Leg}

The results of the outcome parameters are displayed in Figure 5.1 and Table 5.2. In both prosthetic knee conditions, similar differences were found.

The duration of the APA phase was significantly longer when leading with the intact leg. Next to this, the decoupling of the XcoM and CoP during the APA phase was higher for both the leading and trailing leg during the leading intact leg condition. In addition, the impulse 
of the leading intact leg during the APA phase was significantly larger when compared to the impulse of the leading prosthetic leg. For the Rheo Knee II condition, the impulse of the trailing (intact) leg in the leading prosthetic leg condition was significantly higher than the impulse of the trailing (prosthetic) leg in the leading intact leg condition. The CoM velocity at the end of the APA phase was significantly higher in the leading intact leg condition when compared to the leading prosthetic leg condition.

The duration of the EXE phase was significantly shorter for the leading intact leg condition. For the Rheo Knee II condition, the decoupling of the CoM/XcoM and the CoP during the leading intact leg condition were significantly higher when compared to the leading prosthetic leg condition. For the non-microprocessor-controlled prosthetic knee condition, the step length of the leading prosthetic leg was significantly longer when compared to the leading intact leg.

Results of the loading response of the leading prosthetic leg show reduced knee flexion at Initial Contact and knee yielding when compared to the leading intact leg.

\section{Influence of prosthetic knee}

The comparison of the leading intact leg condition in both prosthetic knee conditions showed no significant differences. The same was true for the leading prosthetic leg condition in both prosthetic knee conditions. All $P$ values were 0.11 or over.

\section{Comparison of the Leading Intact Leg condition with controls}

Comparison of the leading intact leg condition of both prosthetic knee conditions to the data of controls show similar results. We found that the duration of the APA phase is significantly increased. The decoupling of the CoM and CoP of the trailing (prosthetic) leg is reduced when compared to the trailing leg of controls. In addition, the impulse of the trailing (prosthetic) leg is reduced when compared to the trailing leg of controls. The duration of the EXE phase is significantly shorter for the leading intact leg condition when compared to controls. The decoupling of the CoM and CoP of the trailing (prosthetic) leg is also reduced when compared to controls. Finally, the step length and CoM velocity at the end of the first step were reduced for the leading intact leg condition when compared to controls.

\section{Comparison of the Leading Prosthetic Leg condition with controls}

The Leading Prosthetic Leg condition showed when compared to controls. Exemptions were the duration of the APA phase, the impulse of the trailing (intact) leg during the APA and EXE phase. On these outcomes no differences were found. In addition, the duration of the EXE phase was significantly longer in the Leading Prosthetic Leg condition. 

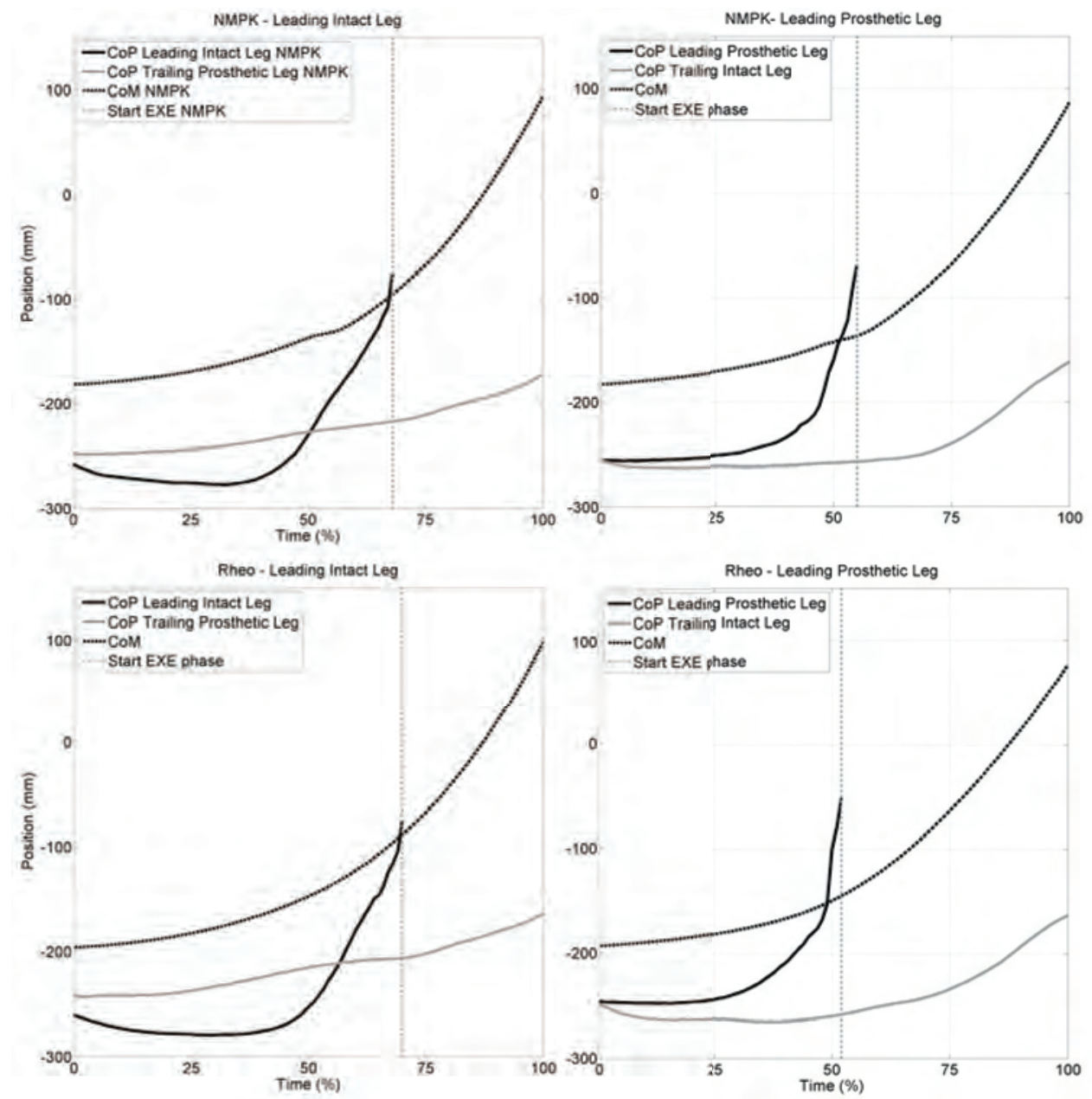

Figure 5.1: Displacement of the center of pressure and center of mass.

On the $x$-axis the timing is displayed. This is the normalized time in which $0 \%$ respresents start of gait initiation and $100 \%$ is initial contact of the leading leg. On the $y$-axis the position of the outcome measures relative to the lab origin is.

Abbreviations: CoP: center of pressure; CoM: center of mass; NMPK: non-microprocessor-controlled prosthetic knee; EXE: step execution phase. 


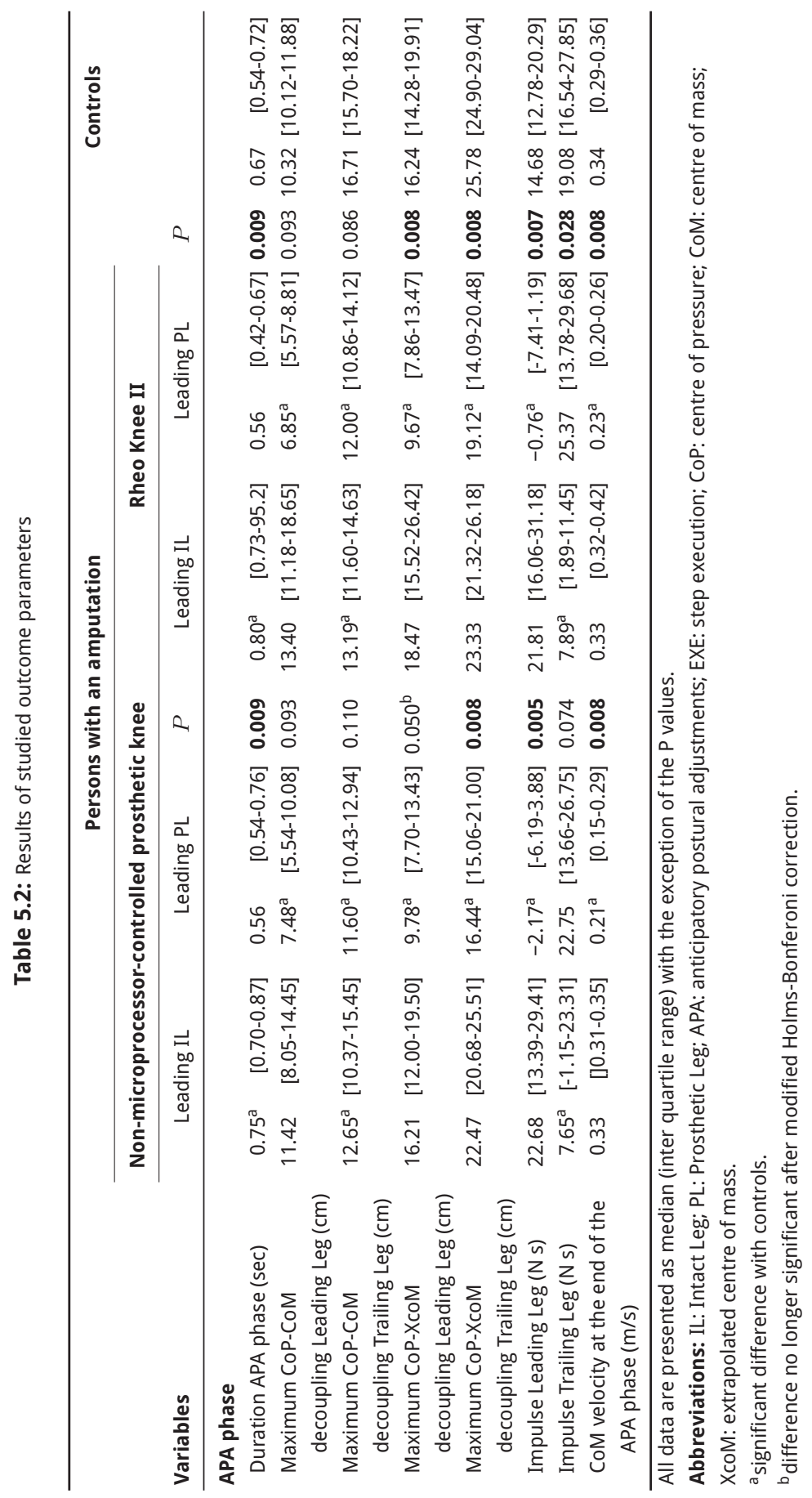




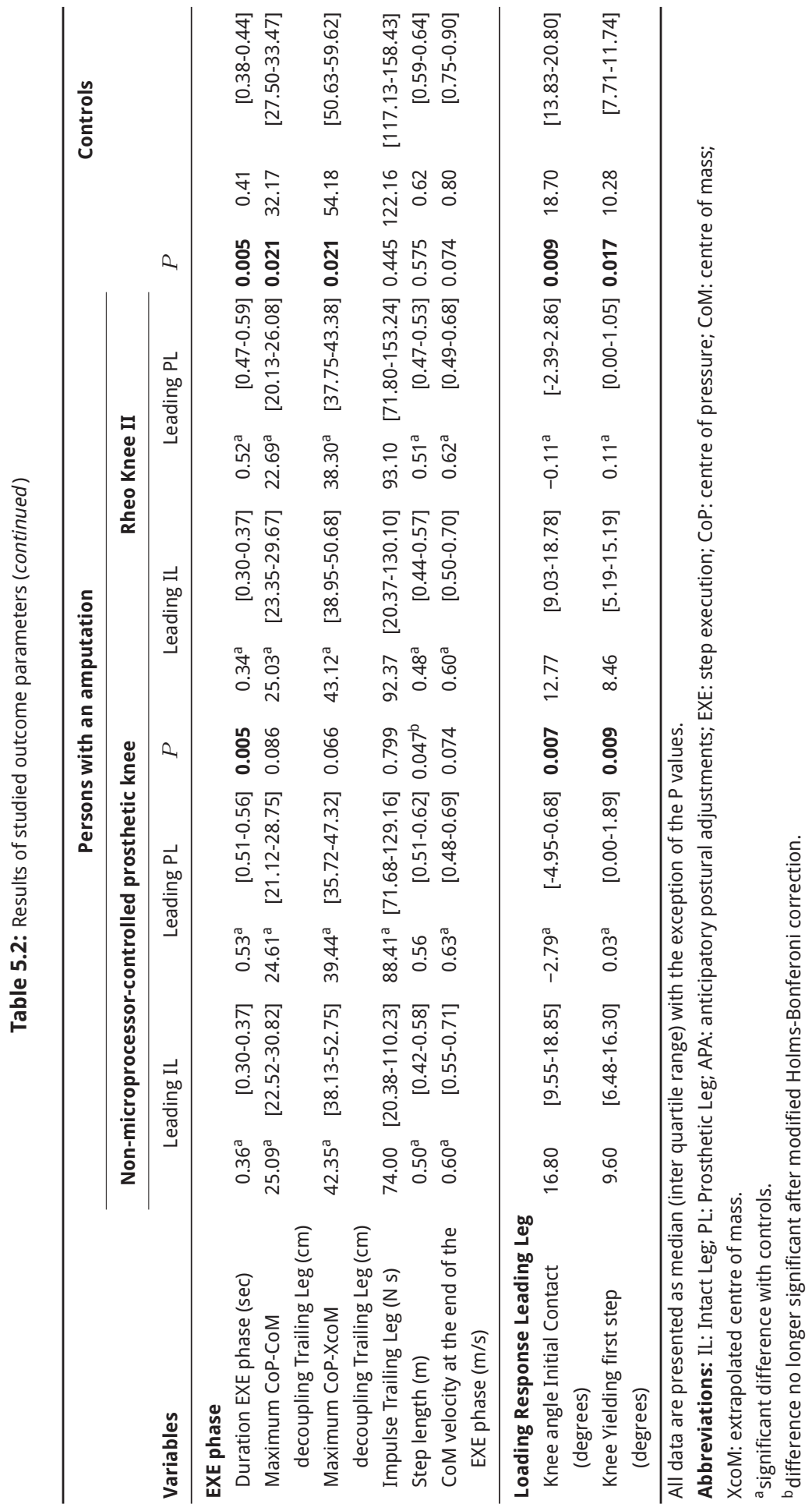




\section{Discussion}

The aim of this study was to investigate the added value of a user-adaptive prosthetic knee in planned gait initiation. We hypothesized increased decoupling of the CoP and CoM in the Rheo Knee II condition by increasing the EXE phase in the leading intact leg condition. In the leading prosthetic leg condition we hypothesized a larger decoupling because of increased stance stability of the prosthetic leg after the first step. Our results disproved our hypotheses and showed that there is no added value of a user-adaptive prosthetic knee during gait initiation.

In the leading intact leg we thought that the single limb support phase of the trailing prosthetic leg would increase while walking with the Rheo Knee II because of increased stance stability. The hypothesized increase of the single limb support of the trailing prosthetic leg would allow a longer period of force production and a longer first step, both of which could lead to a more anterior position of the CoM with respect to the CoP. We, however, did not find an increase in the single limb support phase of the trailing prosthetic leg while walking with the Rheo Knee II as this should be reflected in the EXE phase duration. On this outcome measure, no difference between prosthetic knees was found. In addition, the step length of the first step did not change between prosthetic knee conditions. As a result, the decoupling of the COP and COM was unaffected by the prosthetic knee condition. During the APA phase of the leading intact leg condition also no added value of the Rheo Knee II was found, which was in line with our expectations. Because a prosthetic ankle has no active function, a posterior translation of the CoP is not possible. Changing the prosthetic knee has no influence on this. However, we do feel that our results of the leading intact leg condition provided additional insights into the intact leg reliance. Because of the reduced posterior translation of the CoP under the prosthetic foot, the trailing prosthetic leg has a limited role in the production of propulsive forces. This is most evidently visible in the impulse generated by the leading and trailing leg. In controls, the trailing leg generates a higher impulse than the leading leg. The leading intact leg condition shows the opposite pattern: the leading intact leg produces a significantly higher impulse when compared to controls. The impulse generated by the trailing prosthetic leg is substantially smaller when compared to the trailing intact leg in the leading prosthetic leg condition and controls. For the Rheo Knee II condition this difference was statistically significant, for the non-microprocessor-controlled prosthetic knee this difference was on the borderline of significance. The reduced impulse generated by the prosthetic leg probably led to an increased duration of the APA phase found in the leading intact leg condition. Vrieling et al. explains this by pointing out the dual role of the intact leg in the leading intact leg condition: it must both produce the majority of the propulsive forces and be placed forward first. ${ }^{148}$ Increasing the duration of the APA phase in the leading intact leg condition seems an effective adjustment strategy, as the CoM velocity at the end of the APA phase is comparable to the CoM velocity of controls. 
In the leading prosthetic leg condition, we hypothesized an increased decoupling of the CoP and CoM. This hypothesis was based on the fact that the Rheo Knee II allows increased stance stability and early stance flexion which serves as a shock absorption. We therefore believed that participants could cope with a larger decoupling of the CoP and CoM while starting with the prosthetic leg. Our results disproved our hypothesis as no increase in decoupling of the CoP and CoM was found. A possible explanation is that participants did not use the yielding function of the Rheo Knee II. Previous trials studying the added value of a microprocessor-controlled prosthetic knee in level walking also showed that participants did not use knee yielding function. ${ }^{12,152}$

We did find differences between the leading prosthetic condition on one side and the leading intact leg condition and controls on the other side. During the APA phase, the decoupling of the COM and COP of both the leading prosthetic and trailing intact leg are reduced when compared to controls. When looking at the impulse generated by the leading (prosthetic) leg, it is clear that the prosthetic leg has no role in the production of propulsive forces. It even produces a small negative impulse, meaning that it actually absorbs energy. Again, the intact leg overcompensates for the limited involvement of the prosthetic leg in generating propulsive forces. The impulse of the trailing intact leg is significantly higher when compared to the trailing prosthetic leg in the leading intact leg condition and to the trailing leg of controls. In the Rheo Knee II condition the impulse of the trailing intact leg was also higher than the impulse of the trailing prosthetic leg in the leading intact leg condition. For the non-microprocessor-controlled prosthetic knee condition these differences were on the borderline of significance. Despite the overcompensation of the trailing intact leg, the CoM velocity at the end of the APA phase is smaller when compared to the leading intact leg condition and controls.

The EXE phase was significantly increased in the leading prosthetic leg condition when compared to both the leading intact leg condition and controls. This could be explained by the fact that the prosthetic leg has to absorb the forces that are associated with gait initiation. Because the majority of the non-microprocessor-controlled prosthetic knee are limited in allowing early stance flexion, the prosthetic knee has to be fully extended. In case full knee extension is not achieved before the leading prosthetic leg stance phase starts, the knee might buckle leading to a fall. Achieving full extension of the prosthetic knee probably took more time than achieving the desired knee position of the leading intact leg or the leading leg of controls, which were both slightly flexed. Our results are in line with previously conducted trials, who also found an increase duration of the EXE phase. ${ }^{148}$ In addition, we found that the decoupling of the COM and CoP during the EXE phase were reduced when compared to the leading intact leg condition. For the Rheo Knee II condition these differences were significant, while for the non-microprocessor-controlled prosthetic knee condition these differences were on the borderline of significance. A possible explanation for this finding could be the fact that the prosthetic knee was fully extended at the end of the EXE phase. A slightly flexed knee allows a more anterior position of the CoM when 
compared to a fully extended leg. Despite the reduced decoupling, the impulse generated by the trailing (intact) leg is not reduced when compared to the trailing prosthetic leg in the leading intact leg condition or the trailing leg of controls This is explained by the earlier reported increase in EXE phase: forces can be generated over a longer period of time. The CoM velocity at the end of the EXE phase is slightly higher in the leading prosthetic leg condition when compared to the leading intact leg condition. This was also found by previously conducted trials. ${ }^{148,149,151}$

We chose to calculate the decoupling between the XcoM and CoP next to the generally accepted method of using the decoupling of the CoM and CoP as outcome measure. The results indicate that by using the decoupling of the XcoM and CoP differences between the leading intact and leading prosthetic leg condition and between persons with an amputation and controls become more distinct.

Our study was confounded by a number of factors. First of all we had a small sample size, which affected statistical power and the ability to detect differences. However, when marked differences existed between both prosthetic knee conditions, these would have been identified. In addition, we provided eight weeks of acclimatization which might have been too short. In case full acclimatization was not reached, participants might not have been able to use the added functionality of the Rheo Knee II to a full extend. To our best knowledge, there is one paper available describing the duration of adaptation to a new prosthetic knee. ${ }^{156}$ They showed that it took a 26 year old person with a an amputation due to trauma three weeks to achieve a stable gait pattern after prescription of a new nonmicroprocessor-controlled prosthetic knee. If and to what extent these results are applicable to microprocessor-controlled prosthetic knees is unclear. Third, we chose to leave out a gait training program because it is uncommon in the Netherlands to receive training after prescription of a microprocessor-controlled prosthetic knee. This could have contributed to a situation in which the participants were not fully acclimatized to the Rheo Knee II. Finally, we used a mold to standardize foot placement. This was needed to be able to compare both prosthetic knee conditions. However, some subjects pointed out that, if they had a choice, they would have preferred a different foot placement. This might have influenced the studied outcome measures and may limit the validity of the measurements.

In conclusion we found that persons with an amputation heavily rely on their intact leg for the production of propulsive forces. In addition, decoupling of the COM and CoP is less efficient in persons with an amputation, irrespective of the leg that is placed forward first. Finally, there is no added value of a user-adaptive prosthetic knee on gait initiation. 


\section{The influence of a user-}

adaptive prosthetic knee on planned gait termination: $A$ randomized cross-over trial

Prinsen EC, Nederhand MJ, Koopman HFJM, Rietham JS. 


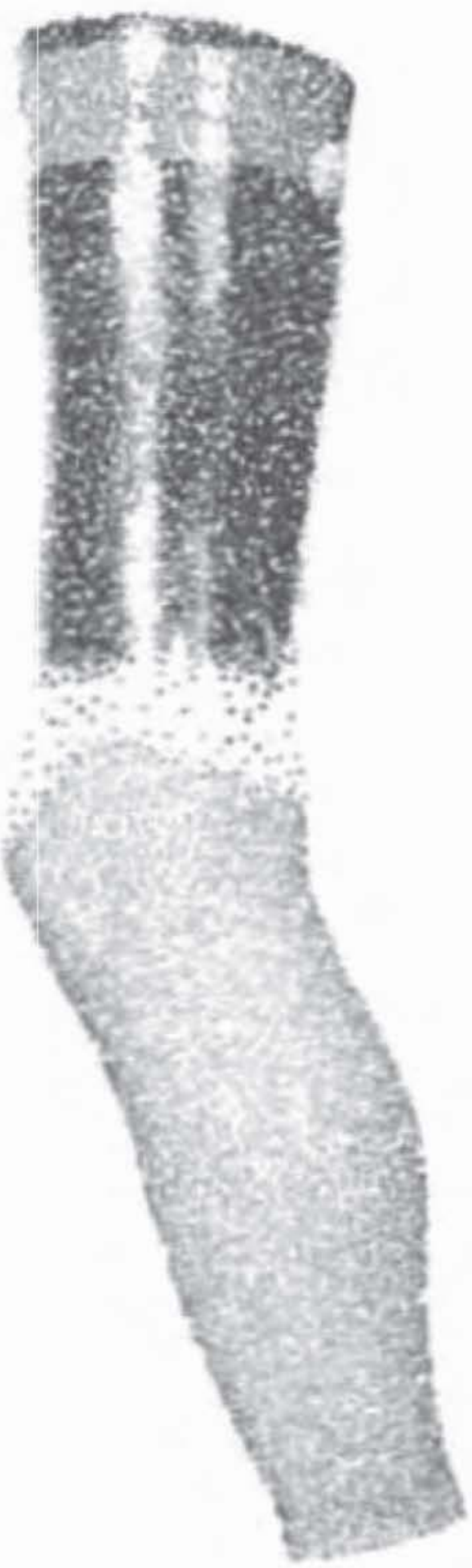




\section{ABSTRACT}

Background The added value of user-adaptive prosthetic knees has been predominantly evaluated in level walking or ramp/stair negotiation. Previous studies indicate that the activity pattern of individuals with an amputation mainly consists of short periods of continuous walking, indicating that a high percentage of ambulatory activity involves gait termination. The potential added value of user-adaptive prosthetic knees in gait termination has not been studied yet.

Methods Ten individuals with an amputation were measured with their own non-microprocessor controlled prosthetic knee and with the Rheo Knee II (a user-adaptive prosthetic knee). The order of testing was randomized. Measurements were performed using a Vicon system and two force plates. Participants performed trials while making the last step with their intact and prosthetic leg. Spatiotemporal, kinematic and kinetic variables were measured.

Findings We found that the Rheo Knee II had no effect on the studied outcome parameters when compared to the non-microprocessor-controlled prosthetic knee. We also found that the intact leg was responsible for producing the deceleration forces irrespective whether the last step was made by the intact or prosthetic leg.

Interpretation The prosthetic leg is limited in producing deceleration forces. Although user-adaptive prosthetic knees claim to increase stance stability and allow knee flexion during early stance, this was not visible in our results. The added value of the Rheo Knee II in gait termination is limited. Future research could focus on the added value of actuated ankle control, which seems to be of greater importance than user-adaptive knee control. 


\section{INTRODUCTION}

Developments in prosthetic knee technology have led to the introduction of user-adaptive microprocessor-controlled prosthetic knees. Examples of these prosthetic knees are the C-Leg and the Rheo Knee. User-adaptive prosthetic knees use information from different sensors that make the knee capable of changing damping properties, such as knee angle, knee angular velocity and force. ${ }^{7,20}$ This variable damping is thought to lead to optimal knee kinematics and, ultimately, be beneficial for the user.

The added value of user-adaptive prosthetic knees has been studied on a number of different tasks, but the majority studied level walking or stair or ramp negotiation. Studies looking into the activity pattern of persons with an amputation showed that majority of periods of continuous activity were only 1 or 2 minutes in length. ${ }^{145}$ Klute et al. also found that persons with an amputation walked for 10 minutes only once or twice a day. ${ }^{145}$ These results indicate that a high percentage of ambulatory activity of persons with an amputation involves gait initiation and termination. A recent study investigated the added value of a user-adaptive prosthetic knee in gait initiation. ${ }^{143}$ However, to our knowledge, the added value of a user-adaptive prosthetic knee in gait termination has not been studied yet.

Previous authors who studied gait termination in persons with an amputation have found that the prosthetic leg has a reduced role in generating deceleration forces that are needed to terminate gait. ${ }^{157,158}$ These deceleration forces are normally generated by placing the center of pressure (CoP) in front of the center of mass (CoM). This slows the forward movement of the CoM down. ${ }^{159}$ Vrieling et al. showed that in persons with a transfemoral amputation this mechanism is impeded. ${ }^{157}$ First of all, the stiffness of the prosthetic ankle inhibits a smooth anterior displacement of the CoP. ${ }^{157}$ Next to this, the absence of prosthetic knee flexion during the loading response inhibits a posterior positioning of the CoM with respect to the CoP. ${ }^{157}$ The lack of knee flexion also inhibits absorption of energy by the knee. This is of particular importance, as the negative work of the knee dissipates the largest amount of energy of any of the lower extremity joints. ${ }^{160}$ Finally, the duration of single limb support of the prosthetic leg is reduced when compared to the intact leg, ${ }^{157,158}$ which limits the time in which the braking impulse can be produced. The duration of single limb support on the prosthetic leg is thought to be decreased because stability is challenged during this phase. Because of all this, persons with an amputation heavily rely on their intact leg for the absorption of energy during gait termination. ${ }^{157,158}$

The Rheo Knee II is thought to increase stance stability. This could increase the duration of single limb support on the prosthetic leg while walking with the Rheo Knee II. This potentially leads to more time to produce deceleration forces and, in turn, to a larger deceleration impulse. Next to this, the Rheo Knee II should allow knee flexion during early stance. This would enable absorption of energy at knee level and would allow a more posterior position of the CoM with respect to the CoP. The latter would mean a more efficient use of the CoP-CoM mechanism. 
The aim of the current study is to investigate the added value of the Rheo Knee II in planned gait termination. Therefore we compared the Rheo Knee II condition with a nonmicroprocessor-controlled prosthetic knee condition. We hypothesized that the prosthetic leg produced a higher deceleration impulse by increasing the duration of the single limb support phase on the prosthetic leg. In addition, we hypothesized increased energy dissipation of the prosthetic knee while using the Rheo Knee II.

\section{METHODS}

\section{Subjects}

For this study, persons with a transfemoral amputation were recruited from the Netherlands and Belgium. The inclusion criteria were: (1) at least one year post amputation, (2) functional level from K2 (limited outdoor) to K4 (active athlete), (3) never supplied with a microprocessor-controlled knee unit before. Exclusion criteria were: (1) other musculoskeletal problems influencing walking ability, (2) problems with the residual leg/bad socket fitting, (3) body weight $>125 \mathrm{Kg}$ (maximum tolerable weight of the Rheo Knee II), (4) knee center-floor distance below $41 \mathrm{~cm}$ (minimal height that is needed to build in the Rheo Knee II).

In addition, 10 persons without an amputation were recruited in the personal network of the primary author to serve as a control group. Controls were eligible when they were free of musculoskeletal problems influencing walking ability, cognitive problems and impaired vision. The study protocol was approved by the Medical Ethical Committee Twente, Enschede, the Netherlands and all subjects provided written informed consent before the start of the measurements.

\section{Study protocol}

The persons with an amputation were randomly assigned to start measurements with their own mechanically passive prosthesis or to start measurements with the Rheo Knee II. We used a block randomization procedure with a block size of four to ensure comparable groups size. We used Excel (Microsoft, Redmond, USA) to generate random numbers which were used for the randomization procedure. In both prosthetic knee conditions the VariFlex ${ }^{\circledR}$ with EVO ${ }^{\mathrm{TM}}$ prosthetic foot was used. Prior to the first measurement, participants with an amputation had an appointment with the prosthesist to either install the Vari-Flex-Evo foot combined with their own prosthesis, or to install the Rheo Knee II in combination with the Vari-Flex with Evo. After eight weeks of acclimatization the first set of measurements was performed. After these measurements, the subjects crossed-over to the other prosthetic condition. After another eight weeks of acclimatization the second set of measurements was performed after which the prosthesis was converted to the original set up and subjects left the study. All prosthetic fittings were performed by the same prosthesist. The 
control group performed one set of measurements. Full details of prosthetic adjustments have been published before. ${ }^{143}$

\section{Experimental protocol}

Participants were instructed to walk five steps before coming to a halt. Data were collected using a three-dimensional optic movement tracking system consisting of six Vicon cameras (Oxford Metrics Ltd., Oxford, UK) and two AMTI force plates (Advanced Mechanical Technology Incorporated, Watertown, USA). The modified Helen-Hayes markers set was used, including 35 reflective makers. Markers were placed on anatomical landmarks according to the Vicon full-body plug in gait model. The collected data were processed using Vicon Nexus 1.8 (Oxford Metrics Ltd., Oxford, UK). The marker trajectories were recorded with a frequency of $100 \mathrm{~Hz}$. The force plate data were collected at a frequency of $1000 \mathrm{~Hz}$. A trial was valid when both the second last step as the step of the leading leg were on a force plate each. For details of the experimental set-up see Figure 6.1. Data collection was stopped when eight valid trials were available. The first five trials with a walking speed within $a \pm 5 \%$ range were selected for analysis.

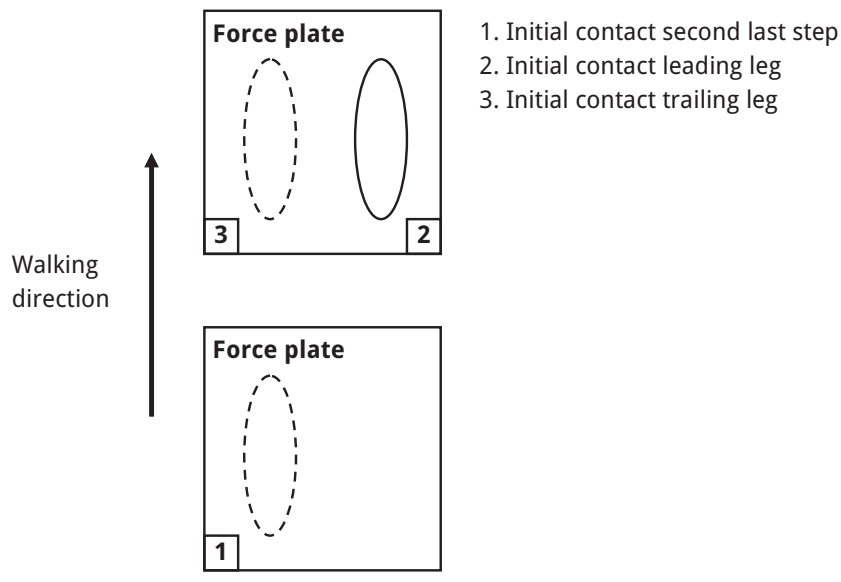

Figure 6.1: Graphical representation experimental set-up

\section{Data processing}

The first step in the data processing was identification of initial contact and initial swing of both legs. Initial contact was defined as the moment the heel marker stopped moving in a downward direction. Initial swing was defined as the moment the heel and toe marker both started moving in the upward direction. Subsequently, data were loaded into custom developed Matlab 2010b software (Mathworks, Natick, USA). Kinematic and kinetic data were filtered using a $2^{\text {nd }}$ order zero phase shift Butterworth filter with a Low-pass filter of $10 \mathrm{~Hz}$. 


\section{Outcome measures}

We calculated spatiotemporal, kinematic and kinetic outcome measures, and the margins of stability. Firstly, spatiotemporal variables included duration of the first double limb support of the leading leg, duration of single limb support on the leading leg, and duration of the moment from initial contact of the trailing leg to end of gait termination. The moment of gait termination was retrospectively defined as the moment the anteroposterior component of the ground reaction force was below $1 \%$ of the body weight in Newton for 100 frames $(0.1 \mathrm{sec})$. Secondly, we compared kinematics of the knee of the leading leg by determining knee angle at initial contact and knee yielding. Knee yielding was defined as the difference between maximum knee flexion during the loading response and knee flexion at initial contact. Thirdly, we calculated joint work of the ankle, knee, and hip of the leading leg as indication of energy dissipation on individual joint level. These were calculated by numerical integration of joint power. In addition, we calculated the deceleration impulse of the second last step and of the leading and trailing leg by numerical integration of the anteroposterior component of the ground reaction force. Finally, we calculated the backward margin of stability from the moment of initial contact of leading leg to the end of gait termination. The backward margin of stability is defined as distance between the CoP and the extrapolated centre of mass (XcoM). ${ }^{155}$ The XcoM is calculated by adding the ratio of the CoM velocity and the eigen frequency of the pendulum to the CoM position. ${ }^{155}$

\section{Data analysis}

Data were analyzed using IBM SPSS statistics 22 software (IBM, Chicago, USA). Because of the small sample size, non-parametric statistics were chosen. For the within-subject comparisons of the persons with an amputation, the Wilcoxon Signed Rank Sum Test was used. For the comparisons of the persons with an amputation with the control subjects, the MannWhitney $\mathrm{U}$ test was used. Statistical significance was set at $P \leq 0.05$.

\section{RESULTS}

\section{Subject recruitment}

A group of 61 potential candidates were contacted of which 52 met the inclusion criteria. A total of twelve persons were willing to participate in the study. Two participants dropped out before the first measurement: one was not able to satisfactorily adjust to the Rheo Knee II and one developed problems with the residual leg. The remaining ten participants completed both measurements. Characteristics of participants and control subjects are displayed in Table 6.1. 
Table 6.1: Descriptive variables participants

\begin{tabular}{lll}
\hline Variables & $\begin{array}{l}\text { Persons with an amputation } \\
(\mathbf{n}=\mathbf{1 0})\end{array}$ & $\begin{array}{l}\text { Control subjects } \\
\text { (n= 10) }\end{array}$ \\
\hline Age (years) & $53(23-67)$ & $28(26-56)$ \\
Sex (male/female) & $6 / 4$ & $5 / 5$ \\
Time since amputation (years) & $33.5(1-41)$ & Not applicable \\
Reason for amputation & Trauma (7), Infection (2), & Not applicable \\
& Osteosarcome (1) & \\
Functional level & K2 (2), K3 (5), K4 (3) & Not applicable \\
Stump length (cm) & $40(32-60)$ & Not applicable \\
Non-microprocessor-controlled & $3 R 60(4), 3 R 80(1)$, Mauch SNS (1), & Not applicable \\
prosthetic knees & Graph Lite (1), CaTech (1), Total & \\
& Knee 2000 (1) & \\
\hline
\end{tabular}

Age, time since amputation, and stump length are presented as median (range).

Sex, reason for amputation, functional level, and non-microprocessor-controlled prosthetic knees are presented as counts

\section{Comparison of leading intact leg and leading prosthetic leg condition within a prosthetic knee condition}

When the leading intact leg condition is compared with the leading prosthetic leg condition within a prosthetic knee condition, similar results are visible for the non-adaptive prosthetic knee and the Rheo Knee II. For results see Table 6.2 and Figure 6.2, and 6.3.

On spatiotemporal variables the following statistically significant differences were found for both the non-adaptive prosthetic knee and Rheo Knee II condition. The duration of the single limb support of the leading leg was significantly larger in the leading intact leg condition, when compared to the leading prosthetic leg condition. In addition, the duration from the moment of initial contact of the trailing leg to the end of gait termination was significantly longer in the leading prosthetic leg condition when compared to the leading intact leg condition. CoM velocity at initial contact of the second-last step, the leading leg, and the trailing leg were also statistically significantly different between the leading intact and leading prosthetic leg condition (see Figure 6.2). For the leading prosthetic leg condition CoM velocity at initial contact of the second-last step (intact leg) and at initial contact of the trailing leg (intact leg) were significantly higher when compared to the leading intact leg condition. CoM velocity at initial contact of the leading leg was significantly higher for the leading intact leg condition when compared to the leading prosthetic leg condition.

On kinematic variables the following statistically significant differences were found in both prosthetic knee conditions. Both the knee angle at initial contact as knee yielding of the leading leg were smaller in the leading prosthetic leg condition when compared to the leading intact leg condition.

On knee work, we found the following statistically significant differences. For the nonadaptive prosthetic knee condition, ankle and hip work of the leading intact leg were higher 

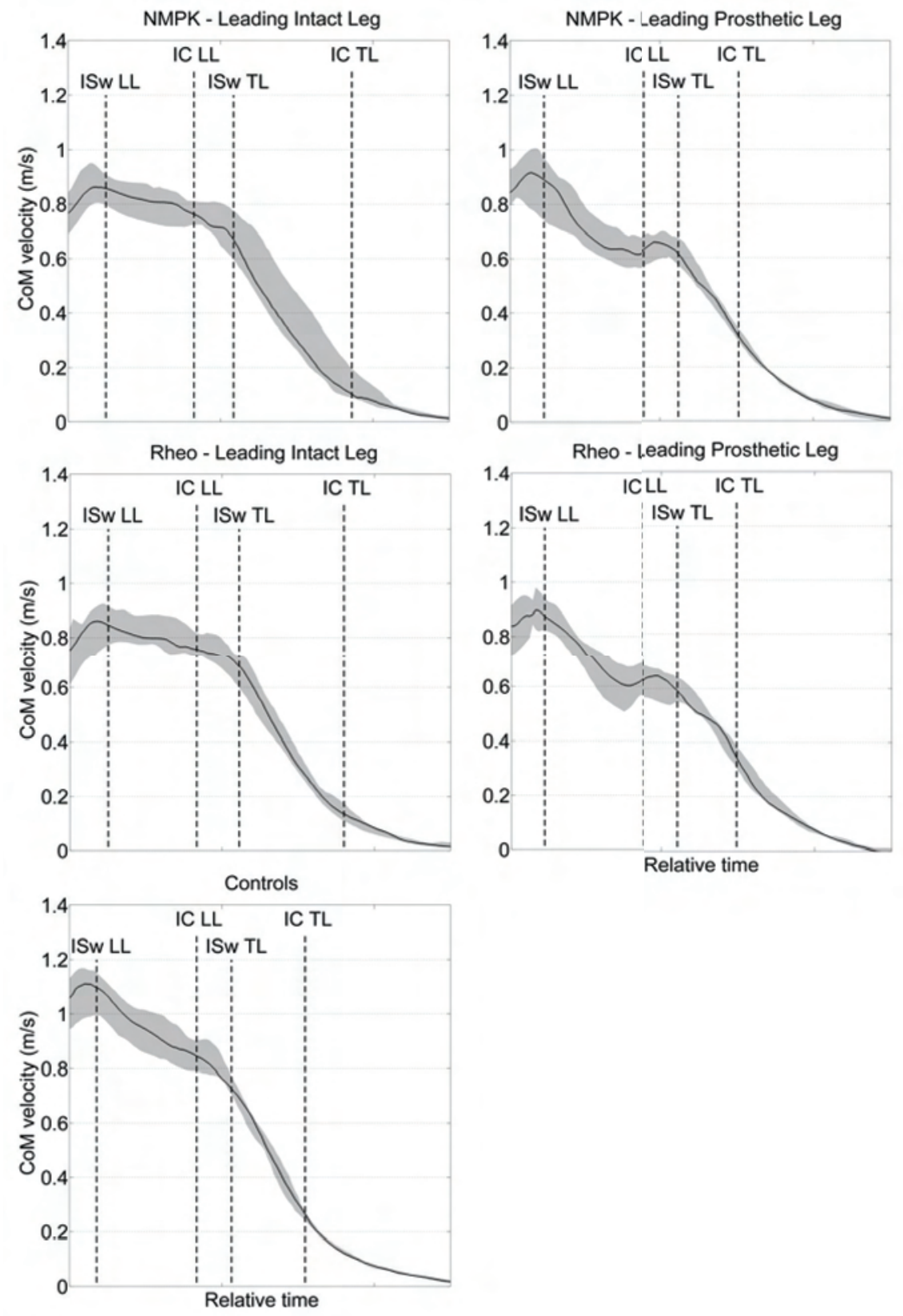

Figure 6.2: Velocity of the center of mass during gait termination.

The black line represents the median velocity of the centre of mass and the gray area represents the interquartile range. The dotted lines from left to right represent: initial swing (ISw) of the leading leg (LL), initial contact (IC) of the leading leg, initial swing of the trailing leg (TL) and initial contact of the trailing leg. The data are scaled in a relative time frame in which the start corresponds with initial contact of the second last step and the end corresponds with the end of gait termination. Abbreviation: NMPK: non-microprocessor-controlled prosthetic knee. 

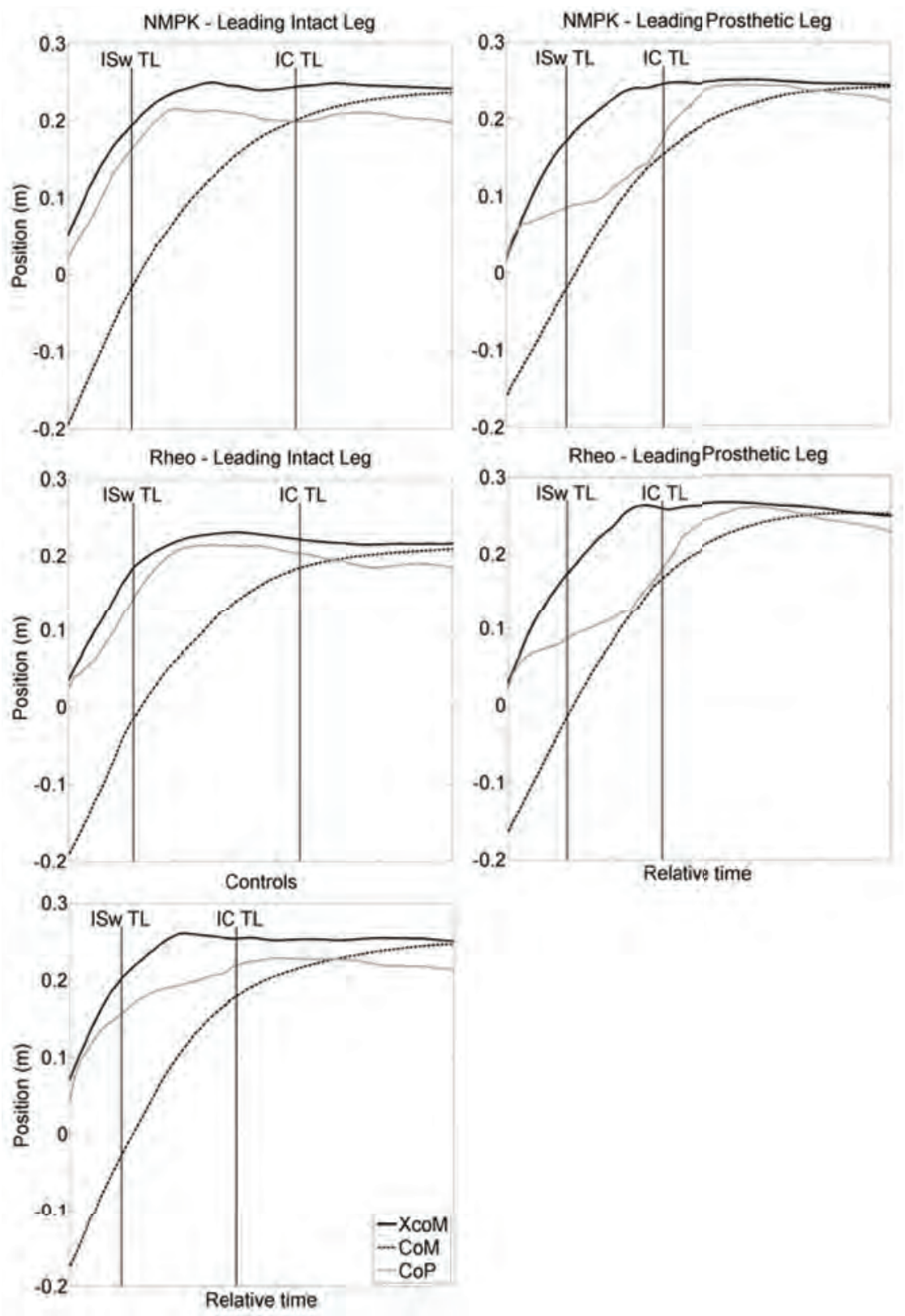

Figure 6.3: Backward margin of stability during gait termination.

The black line represents the median extrapolated centre of mass (XcoM) position, and the gray line the median centre of pressure (COP) position during gait termination. The vertical lines present the moment of initial swing (ISw) and initial contact (IC) of the trailing leg (TL). Positions are relative to the edge of the force plate. The data are scaled in a relative time frame in which the start corresponds with initial contact of the leading leg and the end corresponds with the end of gait termination. Abbreviation: NMPK: non-microprocessor-controlled prosthetic knee. 
when compared to the leading prosthetic leg. For the Rheo Knee II condition, knee work of the leading leg was significantly higher in the leading intact leg when compared to the leading prosthetic leg. When looking at the deceleration impulse the following statistically significant differences were visible for both prosthetic knee condition. For the leading prosthetic leg condition, the deceleration impulse of the second-last step (intact leg) and the deceleration impulse from initial contact of the trailing leg (intact leg) to the end of gait termination were higher when compared to the deceleration impulse produced by the prosthetic leg in the leading intact leg condition. The deceleration impulse of the leading leg was significantly higher in the leading intact leg condition when compared to the leading prosthetic leg condition.

The backward margin of stability is graphically represented in Figure 6.3. For both prosthetic knee conditions, the margins of stability were statistically significantly higher in the leading prosthetic leg condition when compared to the leading intact leg condition.

\section{Comparison of prosthetic knees}

When the leading intact leg condition while walking with the non-adaptive prosthetic knee is compared to the leading leg condition while walking with the Rheo Knee II, no differences were found on all studied outcome parameters. Comparison of the leading prosthetic leg conditions of the non-adaptive prosthetic knee and Rheo Knee II showed one statistically significant difference. Knee flexion of the leading leg at initial contact was significantly higher for the Rheo Knee II condition when compared to the non-microprocessor-controlled prosthetic knee.

\section{Comparison of leading intact leg condition with controls}

For both prosthetic knee conditions, similar results for most outcome parameters were found for the comparison of the leading intact leg condition of the individuals with an amputation and the control subjects. On spatiotemporal variables, we found that the single limb support of the leading intact leg was significantly longer when compared to the control subjects. The duration from initial contact of the trailing leg to the end of gait termination was significantly shorter for the leading intact leg condition when compared to controls. Finally, CoM velocity at initial contact of the second last step and trailing leg (both prosthetic leg) were lower for the individuals with an amputation. On kinematic variables no differences were found. On kinetic variables, we found that the ankle work of the leading intact leg was significantly lower than the ankle work of controls. For the leading intact leg condition we additionally found that the deceleration impulse of the second last step (prosthetic leg) was significantly lower when compared to controls. On margins of stability, no differences were found. 


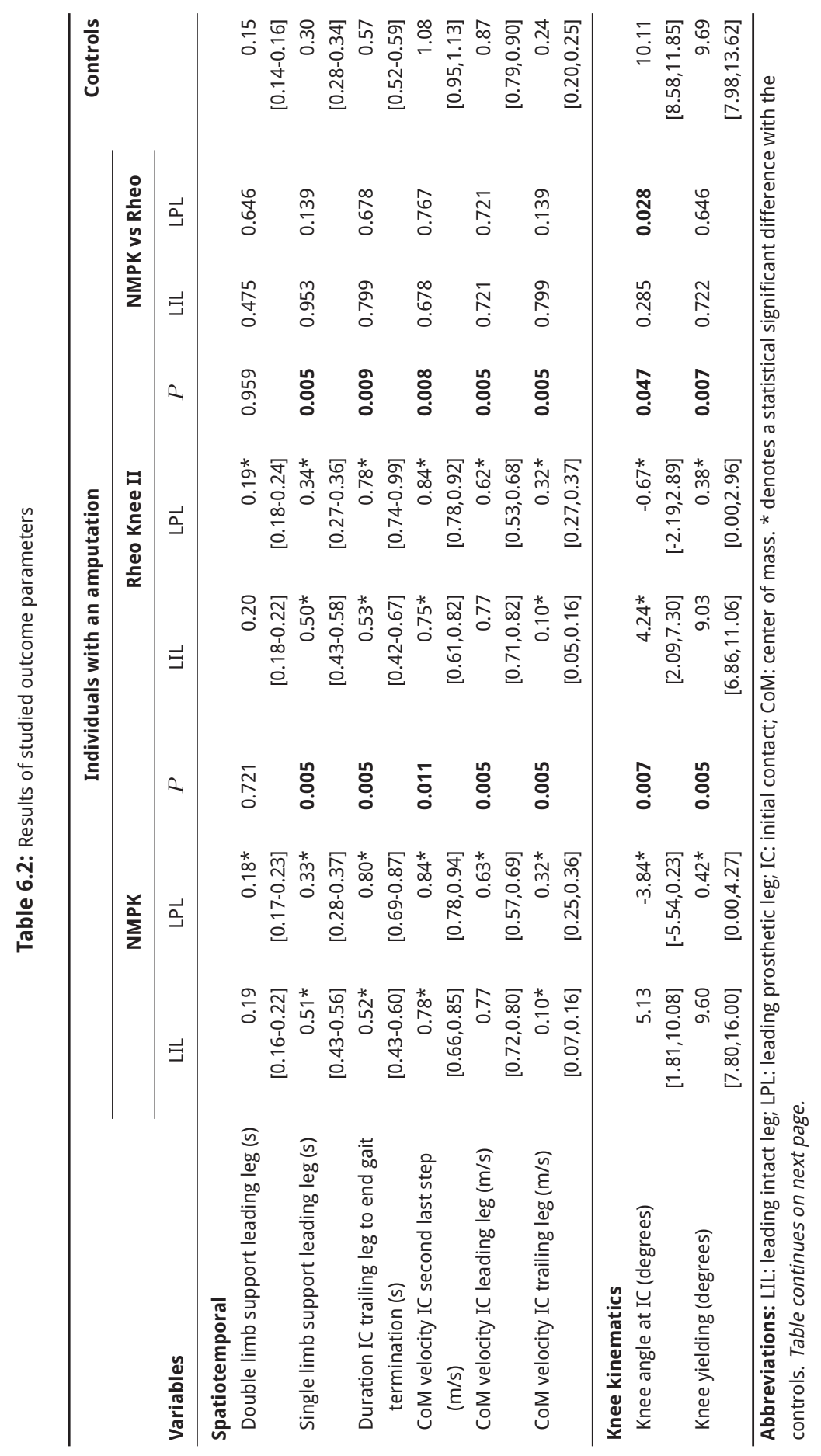




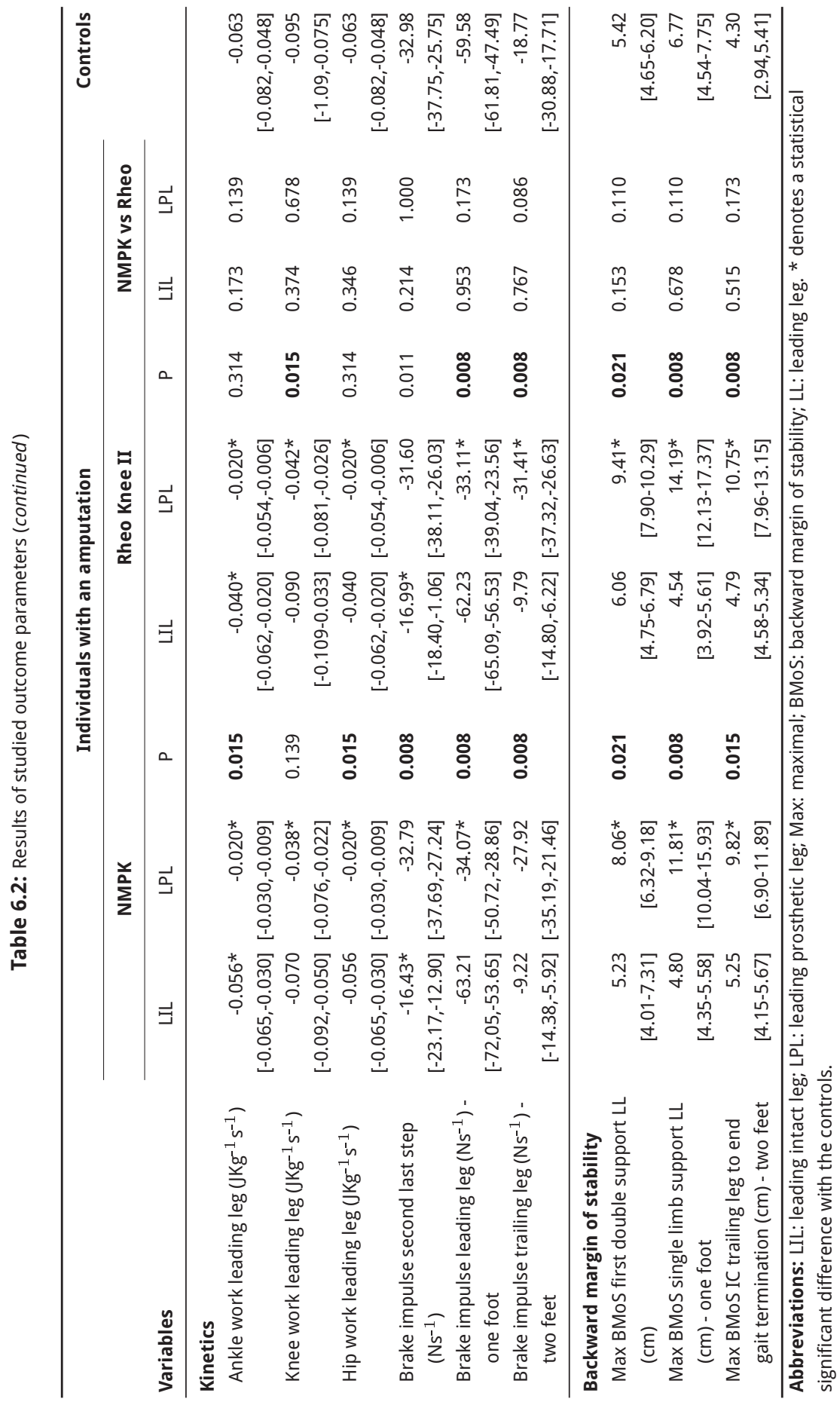




\section{Comparison of the leading prosthetic leg condition with controls}

For both prosthetic knee conditions, statistically significant differences were found for almost all outcome parameters when the leading prosthetic leg condition of the individuals with an amputation is compared to the control subjects. For the non-microprocessorcontrolled prosthetic knee condition, the only non-significant differences were on the deceleration impulse of the second-last step (intact leg) and the deceleration impulse from initial contact of the trailing leg (intact leg) to the end of gait termination. For the Rheo Knee II condition, the non-significant differences were the deceleration impulse of the second-last step (intact leg).

\section{DiscusSION}

The aim of the current study was to study the effect of the Rheo Knee II on biomechanical variables of gait termination. We hypothesized that the prosthetic leg produced a higher deceleration impulse by increasing the duration of the single limb support phase on the prosthetic leg. In addition, we hypothesized increased energy dissipation of the prosthetic knee during the final step while using the Rheo Knee II. Our results disproved our hypotheses and showed that the transition towards the Rheo Knee II had a very limited effect on the studied outcome parameters.

When looking at the first hypothesis, we found that the duration of single limb support of the leading leg was significantly higher in the leading intact leg condition when compared to the leading prosthetic leg condition. This was also found by previous studies. ${ }^{157,158}$ The Rheo Knee II had no influence on the duration of single limb support of the prosthetic leg in both the leading intact and leading prosthetic leg condition when compared to the nonmicroprocessor-controlled prosthetic knee.

It is thought that individuals with an amputation shorten the single limb support duration on the prosthetic leg because stability is challenged during this phase due to the lack of active ankle control. ${ }^{161}$ When looking at the backward margin of stability, we found that the maximal backward margin of stability was significantly higher in all phases of the leading prosthetic leg condition when compared to the leading intact leg condition and controls. This was seen for both the non-microprocessor-controlled prosthetic knee and Rheo Knee II condition. The main reason for the increased backward margin of stability is the reduced anterior shift of the CoP under the prosthetic foot in the leading prosthetic leg condition (see Figure 6.3). A positive backward margin of stability indicates that the CoM will pass the $\mathrm{CoP}$ in the anteroposterior direction. In this light, the increased backwards margin of stability in all phases of the leading prosthetic leg condition further underlines that the prosthetic leg has a limited contribution to slow down the anteroposterior center of mass velocity.

The fact that the CoP-CoM mechanism is impaired in the prosthetic leg was also clearly visible in the deceleration impulses generated by the intact and prosthetic leg. In the control 
subjects, the leading leg was mostly responsible for the generation of the deceleration impulse. This was also the case for the intact leg in the leading intact leg condition. However, the deceleration impulse of the intact leg related to total deceleration impulse (combined deceleration impulse of the leading and trailing leg) was considerably higher in the leading intact leg condition when compared to controls ( $87 \%$ for both prosthetic knee conditions vs. $76 \%$ for the controls). A previous study found that the leading intact leg of individuals with an amputation accounted for $85 \%$ of the total impulse. ${ }^{150}$ Individuals with an amputation seem to increase the deceleration impulse of the leading intact leg by increasing the duration of the single limb support of the leading leg. This duration was significantly longer for the leading intact leg condition when compared to controls. This was also found by Vrieling et al. ${ }^{157}$

In the leading prosthetic leg condition the trailing intact leg also had a substantial role in the generation of the deceleration impulse. The deceleration impulse after initial contact of the trailing intact leg was significantly higher in the leading prosthetic leg condition when compared to both the leading intact leg condition and controls. Contrastingly, the deceleration impulse of the leading prosthetic leg was significantly lower when compared to the leading intact leg and controls. When looking at deceleration impulse of the leading prosthetic leg related to the total deceleration impulse, we found that this was $55 \%$ for the non-microprocessor-controlled prosthetic knee condition, and 51\% for the Rheo Knee II condition. These values are in line with those of van Keeken et al., ${ }^{150}$ also reporting 55\% in their study. In the leading prosthetic leg condition, individuals with an amputation seem to place their trailing intact leg as soon as possible, after which the majority of deceleration forces are produced.

The reliance on the intact leg for the generation of braking forces led to distinct differences in CoM velocity patterns between the leading intact and the leading prosthetic leg condition (see Figure 6.2). Individuals with an amputation seem to lower their CoM velocity before initial contact of the prosthetic leg during the last two steps. This was found irrespective of which leg was leading.

Our second hypothesis was that energy dissipation of the prosthetic knee would increase while walking with the Rheo Knee II compared to walking with the non-microprocessor-controlled prosthetic knee. Our results disproved our hypothesis, as no differences in knee work were seen in the leading prosthetic leg between the non-microprocessorcontrolled prosthetic knee and Rheo Knee II condition.

We did find that intact leg reliance was reflected on all joint work outcome parameters. On all joint levels, the joint work of the leading intact leg was higher than the leading prosthetic leg. Although differences were marked, they were not significant for all comparisons. For the non-microprocessor-controlled prosthetic knee condition, ankle and hip joint work of the leading leg were significantly higher in the leading intact leg condition when compared to the leading prosthetic leg condition. In the Rheo Knee II condition, only the knee work of the leading leg was significantly higher in the leading intact leg condition when com- 
pared to the leading prosthetic leg condition. The joint work of the leading prosthetic leg were comparable in both prosthetic knee conditions.

Comparison of the leading prosthetic leg with controls showed statistically significant lower joints work for the leading prosthetic leg in both prosthetic knee conditions. Next to the difference in duration of single limb support, limited anterior CoP shift and lower gait termination velocity, the reduced knee yielding of leading prosthetic leg contributed to these differences. The reduced knee yielding impairs the mechanism by which the prosthetic knee can absorb energy. Because knee flexion and hip flexion are coupled in a closed chain, the extended prosthetic knee also inhibits hip flexion. By doing so, the hip of the prosthetic leg is also limited in absorbing energy. Because participants did not use the yielding function of the Rheo Knee II, joint work of the leading prosthetic leg did not increase in this prosthetic knee condition. The fact that participants did not use the yielding function might not come as a surprise, as this was also found in trials studying level walking with the C-Leg or Rheo Knee II. ${ }^{11,12,144}$ We did find that the knee angle at initial contact was significantly higher in the Rheo Knee II condition. However, we consider this finding as not relevant as differences were small and they could be due to differences in alignment between the non-microprocessor-controlled prosthetic knee and the Rheo Knee II.

We believe that a number of factors played a role in the limited differences that we found between prosthetic knee conditions. First of all, we did not provide a training program when walking with the Rheo Knee II. As the majority of non-microprocessor-controlled prosthetic knee do not allow early stance flexion, the majority of the participants learned to walk with an extended knee throughout the stance phase during their rehabilitation. It is doubtable whether participants were able to unlearn this walking pattern without training. As it is not common in the Netherlands to provide a training program after provision of a microprocessor-controlled prosthetic knee we chose to stick to usual care as closely as possible. Next to this, we had a small study population which affected statistical power which makes it harder to find statistically significant differences. However, if marked differences between prosthetic knee conditions were present, these could have been identified. Secondly, users had eight weeks to get accustomed to the Rheo Knee II which might have been too short for full customization. Especially because we compared the Rheo Knee II to an extensive experience with the non-microprocessor-controlled prosthetic knee.

Future research could focus on the added value of an active prosthetic foot or commercially available passive prosthetic feet where the passive plantar flexion can be reduced that might be able to shift the CoP to a more anterior position under the prosthetic foot. If so, individuals with an amputation might be able to use the CoP-CoM mechanism by which braking forces are generated to a greater extent.

In conclusion, individuals with a transfemoral amputation or knee disarticulation heavily rely on the intact leg to stop the forward momentum needed to terminate gait. In addition, the Rheo Knee II does not reduce intact leg reliance and has no added value during gait termination when compared to non-microprocessor-controlled prosthetic knees. 
Responses of individuals with

\section{an amputation to posterior platform perturbations during walking: Influence of a user- adaptive prosthetic knee}

Prinsen EC, Nederhand MJ, Prins MR, Koopman HFJM, Rietman JS. Submitted to Arch Phys Med Rehab. 


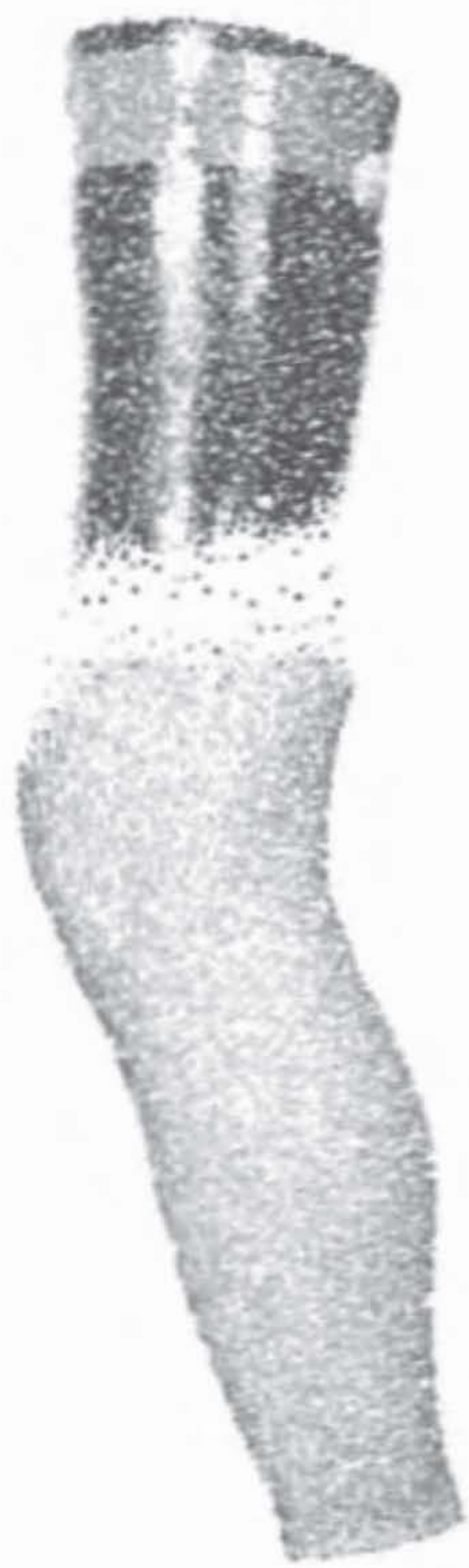




\section{Abstract}

Background The use of microprocessor-controlled prosthetic knees has been associated with a reduced number of self-reported falls and stumbles. A biomechanical explanation for this finding, however, is lacking. The aim of this study was to compare the use of a non-microprocessor-controlled prosthetic knee and the Rheo Knee II on responses to anteroposterior platform perturbations during walking.

Methods Participants were measured twice: once with their own non-microprocessor-controlled prosthetic knee and once with the Rheo Knee II. We measured both perturbed and non-perturbed walking, both at preferred walking speed. A control group was included for reference purposes. Anteroposterior platform perturbations were applied during the single stance phase on the prosthetic leg and during the end of the swing phase of the prosthetic leg. Primary outcome measure was the backward margin of stability (BMoS). Secondary outcome measures included the mediolateral margin of stability, anteroposterior center of mass velocity, step length, and foot forward placement.

Results The BMoS of the steps after the stance phase perturbations in the Rheo Knee II condition was significantly increased when compared to the non-microprocessor-controlled condition. This is explained by a smaller foot forward placement in the Rheo Knee II condition. For the BMoS of the steps after the swing phase perturbations no differences were found. Comparison of perturbed and non-perturbed walking showed that the Rheo Knee II enabled the use of strategies that are also used by non-amputees to cope with the balance perturbations.

Conclusions The use of the Rheo Knee II led to an increased BMoS after the stance phase perturbations which is thought to be reflective of a decreased fall risk. The design of the Rheo enabled the use of strategies that were also used by non-amputees. This study provided some initial biomechanical findings that might explain the reduced self-reported stumbles and falls that have been described before while using microprocessor-controlled prosthetic knees. 


\section{INTRODUCTION}

Falling is highly prevalent in individuals with an amputation. A survey conducted in 2001 showed that over $66 \%$ of respondents with a transfemoral amputation fell at least once in the past 12 months. ${ }^{14}$ Next to this, fear of falling has been shown to be deteriorate daily functioning as it has been shown to reduce prosthetic use ${ }^{162}$ and engaging in social activities. ${ }^{163}$ Because of this, it is of clinical importance to understand the responses of individuals with a transfemoral or knee disarticulation amputation to balance perturbations and how interventions can affect these responses.

When looking in the scientific literature it becomes clear that there is a limited body of evidence regarding the responses of individuals with a transfemoral amputation to balance perturbations during walking. Shirota et al. studied the responses of individuals with a transfemoral amputation and control subjects on swing phase perturbations of both the prosthetic and intact leg. ${ }^{164}$ They found that individuals with a transfemoral amputation generally used recovery strategies also used by individuals without an amputation. ${ }^{164}$ In addition, they found the biggest differences between individuals with and without an amputation during tripping of the intact leg. ${ }^{164}$ Sheehan et al. ${ }^{165}$ and Crenshaw et al. ${ }^{166}$ investigated the effect of a perturbation-based gait training program and found that this could improve compensatory stepping response and margins of stability. As far as it is known, no attempts have been made to investigate the effect of different prosthetic components on the responses to balance perturbations during walking.

An example of a prosthetic component that might influence the responses to balance perturbations are microprocessor-controlled prosthetic knees. Microprocessor-controlled prosthetic knees are designed to increase stability during early stance, provide optimal knee kinematics during swing, and to adapt to changes in walking speed., 7,20 There are indications that the use of a microprocessor-controlled prosthetic knees result in a lower number of subject-reported stumbles and falls when compared to the use of non-microprocessorcontrolled prosthetic knees. ${ }^{26-28,32}$ There is, however, no biomechanical explanation available for these observed differences. One possible explanation could be found in previous research studying the responses to platform perturbations. Hak et al. found that both individuals with and without a transtibial amputation decreased step length and increased step frequency to increase stability when confronted with platform perturbations. ${ }^{167,168}$ Decreasing step length could be difficult for individuals walking with a non-microprocessorcontrolled prosthetic knee as the knee has to be extended at the beginning of the stance phase to avoid knee buckling. Achieving full prosthetic knee extension requires a relatively large step length. Because microprocessor-controlled prosthetic knee allow early stance knee flexion, individuals might decrease prosthetic step length without jeopardizing knee stability. When this is the case, individuals with a transfemoral amputation or knee disarticulation might use similar strategies that non-amputees use to increase stability. This hypothesis, however, has not been confirmed. 
The aim of this study was therefore to study to compare the responses of individuals with a transfemoral amputation or knee disarticulation to balance perturbations while walking with a non-microprocessor-controlled prosthetic knee and the Rheo Knee II. We also investigated whether the prosthetic knee influenced the strategies that were used during perturbed walking when compared to non-perturbed walking and how these strategies relate to the strategies used by individuals without an amputation. Because of the increased early stance stability that is attributed to the use of microprocessor-controlled prosthetic knees we hypothesized that the use of the Rheo Knee II would be beneficial in terms of margins of stability. We also hypothesized that the Rheo Knee II would enable the use of strategies that were also used by individuals without an amputation.

\section{METHODS}

\section{Subjects}

We recruited participants from the service area of Roessingh Center for Rehabilitation, Enschede, the Netherlands, and through a flyer on a national website of a patient organization. Inclusion criteria were: (1) at least one year post amputation; (2) functional level from K2 (limited outdoor) or higher ${ }^{142}$; (3) never previously fitted with a microprocessor-controlled knee. Exclusion criteria were: (1) other musculoskeletal problems influencing walking ability; (2) stump problems / poor socket fitting; (3) body weight > $125 \mathrm{Kg}$ (maximum specification weight for the Rheo Knee II); (4) knee center-floor distance below $41 \mathrm{~cm}$. In addition, a control groups of subjects without an amputation were recruited for reference purposes. The controls were free of any musculoskeletal problems influencing walking ability.

The study protocol (NL 30112.044.09) was approved by the Ethical Research Committee Twente, Enschede, the Netherlands and all subjects provided written informed consent before the first prosthetic adjustment.

\section{Prosthetic adjustments}

The full process of prosthetic adjustments have been published before. ${ }^{143}$ To prevent a learning effect, we randomized the order in which the non-microprocessor-controlled prosthetic knee and Rheo Knee II were tested. To rule out the effect of difference in prosthetic feet between prosthetic conditions and/or participants, we used the LP Vari-Flex ${ }^{\circledR}$ with EVO ${ }^{\mathrm{TM}}$ (Össur) in both prosthetic knee conditions. During the first set of prosthetic adjustments, either the LP Vari-Flex with EVO was installed or the Rheo Knee II in combination with the LP Vari-Flex with EVO. The first data collection took place after eight weeks of adjustment time. During the second set of prosthetic adjustments, participants went back to their own nonmicroprocessor-controlled prosthetic knee or got the Rheo Knee II installed. The second data collection took place after another eight weeks of adjustment time. After the second data collection, the prosthesis was converted back to the original set-up after which partici- 
pants left the study. Participants did not receive a gait training program in either prosthetic knee condition. This choice was based on our aim to follow usual care as closely as possible, which in the Netherlands usually does not include a training program after the prescription of a microprocessor-controlled prosthetic knee.

\section{Protocol}

Data were collected at the Center for Augmented Learning and Training of the National Military Rehabilitation Center Aardenburg in Doorn, the Netherlands using the ComputerAssisted Rehabilition Environment (CAREN) system (Motek Forcelink BV, Amsterdam, the Netherlands). This CAREN system consists of an instrumented single-belt treadmill and a twelve infrared-camera Vicon motion capture system (Oxford Metrics Ltd., Oxford, UK). The treadmill speed was fixed. We determined preferred walking speed during a familiarization trial in which the walking speed was increased until the subjects were comfortable.

We used the modified Helen-Hayes marker set, including 37 reflective markers, which were placed according to the Vicon full-body Plug-in-Gait model. An additional two markers were placed on the rope that connected the safety harness to the overhead frame. Before the measurements started, we asked participants to place their full weight in the safety harness and recorded the distance between the two markers on the rope. This distance was used to check whether participants made use of the safety harness during the measurements. The strides for which this was the case were omitted from the data analysis. We also placed four markers on the platform which were used to determine the moments of platform perturbations.

The perturbations consisted of a backward translation of the platform with a amplitude of $0.2 \mathrm{~m}$ and a velocity of $0.2 \mathrm{~m} / \mathrm{s}$. Perturbations were applied manually by pressing a button based on visual inspection of the gait cycle during the single support phase of the prosthetic leg or during the end of the swing phase of the prosthetic leg. The moment of single stance on the prosthetic leg was chosen as the balance perturbations has to be controlled by the prosthetic leg alone and was thought to represent a 'slip'. The end of the swing phase was chosen, as we hypothesized that the perturbations would provoke an earlier start of the stance phase on the prosthetic leg and was thought to represent a 'trip'. For the comparison of perturbed to non-perturbed walking we also collected data of non-perturbed walking. We collected these data at the same walking speed that was used during the perturbation trials. A total of 15 strides were analyzed and averaged.

The collected data were processed using Vicon Nexus 1.8 (Oxford Metrics Ltd., Oxford, UK). We loaded the processed data into customized Matlab 2010b software (The MathWorks, Inc, Natick, USA) for further analysis. We filtered the kinematic data using a zero-phase shift $2^{\text {nd }}$ order Butterworth filter with a cut-off frequency of $10 \mathrm{~Hz}$, meaning that the data were filtered twice with a $2^{\text {nd }}$ order filter.

The first step of the data analysis was to determine the timing of the perturbations. In case the perturbations were not applied during the single stance on the prosthetic leg (in 
between toe-off and initial contact of the intact leg) or not during the end of the prosthetic swing phase (defined as $75-95 \%$ of the gait cycle) of the prosthetic leg, the perturbation and the associated outcome parameters were omitted from the data analysis. In case of a valid perturbation, outcome parameters were calculated and averaged per timing of perturbation.

\section{Outcome measures}

We analyzed the outcome parameters of the first six steps after the perturbations because we hypothesized that participants were able to return to their normal walking pattern within these six steps. The primary outcome measure is the backward margin of stability (BMoS), see Figure 7.1. Increased BMoS is thought to be indicative of increased gait stability ${ }^{167} \mathrm{We}$ also chose to calculate the mediolateral margin of stability (ML-MoS) to explore whether anteroposterior perturbations also affects the margin of stability in the mediolateral plane. The choice for the ML-MoS was also based on the fact that previous research showed that the ML-MoS was affected by perturbations other than mediolateral perturbations, such as perturbations of the visual field. ${ }^{169}$ The backward margin of stability was calculated as the difference between the anteroposterior position of the extrapolated center of mass (XcoM) and the anteroposterior position of the marker placed on the lateral malleolus. The XcoM was calculated according to Hof et al. ${ }^{155}$ The mediolateral MoS was calculated as the difference between the mediolateral position of the XcoM and the mediolateral position of the marker placed on the lateral malleolus. .

To understand potential differences in backward margin of stability we calculated secondary outcome measures that are in line with Hak et al. ${ }^{168}$ that could provide an explanation for these differences. The secondary outcome measures include: (1) anteroposterior CoM velocity at initial contact, (2) step length, and (3) foot forward placement. The anteroposterior center of mass velocity was determined by taking the first derivative of the center of mass positions provided by the Vicon Nexus software in intervals of 0.1 seconds. Step length was calculated as the difference in anteroposterior positions of the ankle markers at initial contacts. Foot forward placement was calculated as the distance between the anteroposterior components of the ankle marker and the center of mass position at initial contacts.

\section{RESULTS}

A total of eight individuals with an amputation participated in the study. Of this study group, one individual could not cope with the movement of the platform towards the neutral position, leaving a total of seven individuals who completed both measurement sessions. The control group consisted of ten individuals without an amputation. Demographic variables of the study group are displayed in Table 7.1. 


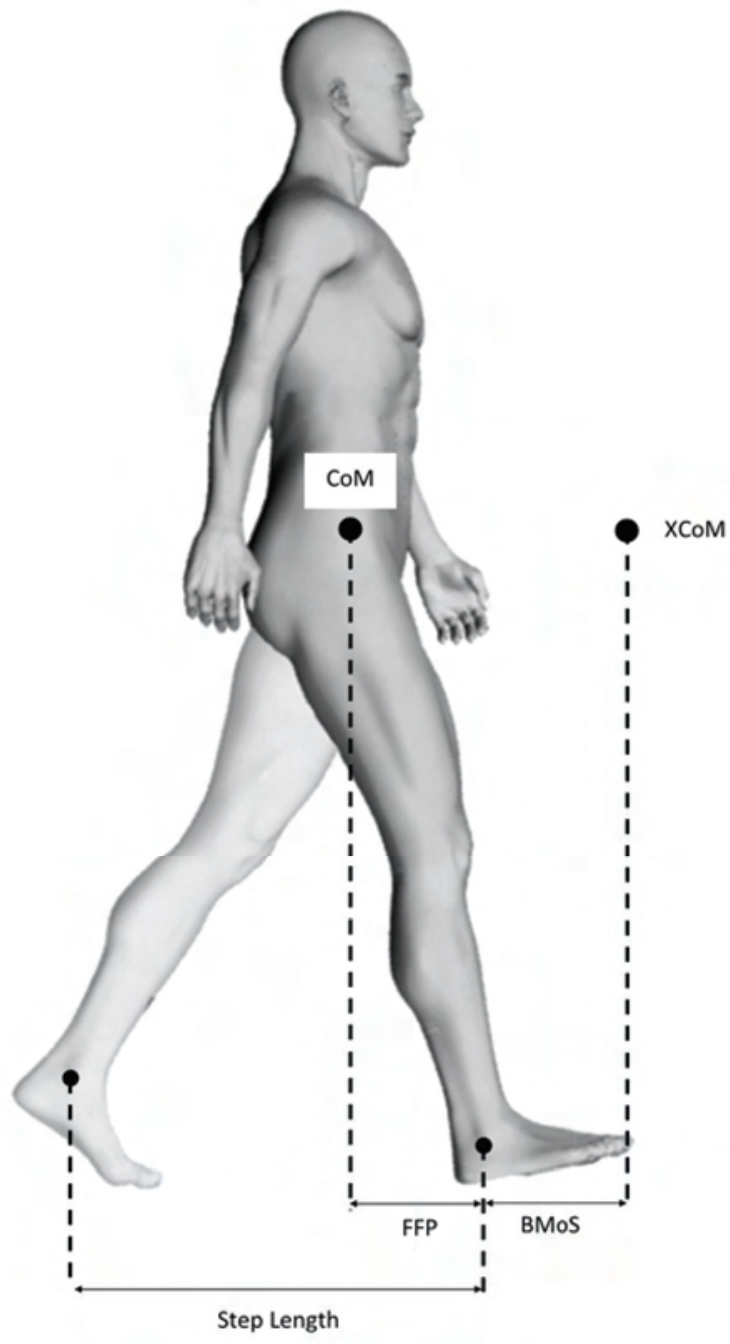

Figure 7.1: Graphical representation of the outcome measures associated with the backwards margin of stability. Abbreviations: CoM: center of mass; XcoM: extrapolated center of mass; FFP: forward foot placement; BMoS: backward margin of stability. 
Table 7.1: Descriptive variables participants

\begin{tabular}{lll}
\hline Variables & $\begin{array}{l}\text { Persons with an amputation } \\
(\mathbf{n}=\mathbf{7})\end{array}$ & $\begin{array}{l}\text { Control subjects } \\
\text { (n= 10) }\end{array}$ \\
\hline Age (years) & $53(23-65)$ & $28(26-56)$ \\
Sex (male/female) & $4 / 3$ & $5 / 5$ \\
Time since amputation (years) & $33.5(1-41)$ & Not applicable \\
Reason for amputation & Trauma (7), Infection (2), & Not applicable \\
& Osteosarcome (1) & \\
Functional level & K3 (4), K4 (3) & Not applicable \\
Stump length (cm) & $34(8-35)$ & Not applicable \\
Non-microprocessor-controlled & 3R60 (4), 3R80 (1), Graph Lite (1), & Not applicable \\
prosthetic knees & CaTech (1) & \\
\hline
\end{tabular}

Age, time since amputation, and stump length are presented as median (range). Sex, reason for amputation, functional level, and non-microprocessor-controlled prosthetic knees are presented as counts

\section{Timing of perturbations}

The timing of perturbations is displayed in Table 7.2. Several perturbations had to be omitted due to incorrect timing. Between five and eight valid perturbations were available for each moment of perturbation per participant per prosthetic knee condition. There were no statistical significant differences in the timing of the perturbations between prosthetic knee conditions.

\section{Comparison of prosthetic knee conditions}

The results of the comparison of prosthetic knee conditions are displayed in Table 7.2, 7.3 and Figure 7.2. The BMoS of the steps after the stance phase perturbations were significantly higher in the Rheo Knee II condition compared to the non-microprocessor-controlled prosthetic knee condition. For the swing phase perturbation no differences were seen in BMoS between prosthetic knee conditions. There were no statistical significant differences in the ML-MoS of the steps following both perturbations between the non-microprocessorcontrolled prosthetic knee and the Rheo Knee II for either the stance or swing phase perturbation.

\section{Secondary outcome measures}

Because we only found differences between prosthetic knee conditions for the BMoS after the stance phase perturbations, only the outcome measures associated with this perturbation timing were compared. For the results see Table 7.3. The anteroposterior center of velocity at initial contact of step 3 (intact leg) was statistically significantly higher in the Rheo Knee II condition when compared to the non-microprocessor-controlled prosthetic knee condition. The step length of step 4 and 6 (prosthetic leg) was significantly smaller in the Rheo Knee II condition when compared to the non-microprocessor-controlled prosthetic 
Table 7.2: Comparison of prosthetic knee conditions

\begin{tabular}{|c|c|c|c|c|c|}
\hline \multirow{2}{*}{$\begin{array}{l}\text { Variables } \\
\text { Stance phase perturbations }\end{array}$} & \multicolumn{2}{|r|}{ NMPK } & \multicolumn{2}{|c|}{ Rheo Knee II } & \multirow[t]{2}{*}{$P$} \\
\hline & & & & & \\
\hline Timing perturbation (\% GC) & 23.34 & {$[16.77,30.22]$} & 21.29 & {$[17.60,22.84]$} & 0.735 \\
\hline BMoS Step 1 (cm) - Intact Leg & 4.84 & {$[2.43,8.78]$} & 7.00 & {$[4.95,9.72]$} & 0.028 \\
\hline BMoS Step $2(\mathrm{~cm})$ - Prosthetic Leg & 5.74 & {$[0.88,11.10]$} & 9.35 & {$[1.86,15.83]$} & 0.043 \\
\hline BMoS Step 3 (cm) - Intact Leg & 8.71 & {$[5.01,11.91]$} & 10.65 & {$[8.46,13.24]$} & 0.028 \\
\hline BMoS Step $4(\mathrm{~cm})$ - Prosthetic Leg & 4.39 & {$[0.42,8.74]$} & 7.69 & {$[5.99,10.64]$} & 0.028 \\
\hline BMoS Step $5(\mathrm{~cm})$ - Intact Leg & 6.39 & {$[5.55,11.63]$} & 10.35 & {$[7.29,12.16]$} & 0.018 \\
\hline BMoS Step $6(\mathrm{~cm})$ - Prosthetic Leg & 6.38 & {$[1.72,8.67]$} & 8.49 & {$[5.78,11.33]$} & 0.018 \\
\hline ML-MoS Step 1 (cm) - Intact Leg & 11.37 & {$[8.49,12.38]$} & 10.77 & {$[9.07,12.39]$} & 0.499 \\
\hline ML-MoS Step 2 (cm) - Prosthetic Leg & 11.21 & {$[7.85,12.79]$} & 9.59 & {$[7.89,11.00]$} & 0.398 \\
\hline ML-MoS Step 3 (cm) - Intact Leg & 10.67 & {$[9.93,11.86]$} & 12.22 & {$[9.02,13.04]$} & 0.237 \\
\hline ML-MoS Step 4 (cm) - Prosthetic Leg & 9.82 & {$[8.63,12.65]$} & 10.48 & {$[8.95,12.38]$} & 0.612 \\
\hline ML-MoS Step 5 (cm) - Intact Leg & 10.43 & {$[8.89,12.49]$} & 11.10 & {$[9.38,12.55]$} & 0.499 \\
\hline ML-MoS Step $6(\mathrm{~cm})$ - Prosthetic Leg & 10.03 & {$[9.06,12.67]$} & 11.08 & {$[8.80,12.48]$} & 0.499 \\
\hline \multicolumn{6}{|l|}{ Swing phase perturbations } \\
\hline Timing perturbation (\% GC) & 90.02 & {$[86.43,92.13]$} & 90.06 & {$[85.83,90.92]$} & 0.398 \\
\hline BMoS Step $1(\mathrm{~cm})$ - Prosthetic Leg & 1.21 & {$[-1.12,9.27]$} & 7.01 & {$[4.87,12.02]$} & 0.063 \\
\hline BMoS Step 2 (cm) - Intact Leg & 6.88 & {$[3.97,8.35]$} & 8.27 & {$[6.51,10.09]$} & 0.237 \\
\hline BMoS Step $3(\mathrm{~cm})$ - Prosthetic Leg & 6.64 & {$[0.08,13.11]$} & 10.00 & {$[4.83,13.60]$} & 0.237 \\
\hline BMoS Step 4 (cm) - Intact Leg & 7.82 & {$[5.58,13.77]$} & 9.27 & {$[7.69,11.91]$} & 0.735 \\
\hline BMoS Step $5(\mathrm{~cm})$ - Prosthetic Leg & 7.95 & {$[1.52,12.04]$} & 10.05 & {$[5.01,13.69]$} & 0.063 \\
\hline BMoS Step 6 (cm) - Intact Leg & 6.91 & {$[6.21,13.17]$} & 10.59 & {$[8.15,12.90]$} & 0.499 \\
\hline ML-MoS Step 1 (cm) - Intact Leg & 11.04 & {$[9.95,12.41]$} & 10.99 & {$[9.04,13.31]$} & 0.398 \\
\hline ML-MoS Step 2 (cm) - Prosthetic Leg & 11.68 & {$[8.91,12.14]$} & 9.85 & {$[8.24,12.15]$} & 0.735 \\
\hline ML-MoS Step 3 (cm) - Intact Leg & 9.92 & {$[9.30,10.80]$} & 11.88 & {$[9.06,13.46]$} & 0.398 \\
\hline ML-MoS Step 4 (cm) - Prosthetic Leg & 10.61 & {$[8.82,13.24]$} & 10.29 & {$[9.63,11.13]$} & 1.000 \\
\hline ML-MoS Step 5 (cm) - Intact Leg & 10.06 & {$[8.91,12.07]$} & 10.31 & {$[8.42,12.48]$} & 0.499 \\
\hline ML-MoS Step 6 (cm) - Prosthetic Leg & 10.64 & {$[8.93,13.64]$} & 10.20 & {$[9.47,11.76]$} & 0.612 \\
\hline
\end{tabular}

Abbreviations: GC: gait cylce; BMoS: backward margin of stability; ML-MoS: mediolateral margin of stability

knee condition. The foot forward placement of step 4 through 6 (resp. intact, prosthetic and intact leg) of the Rheo Knee II condition was significantly smaller when compared to the non-microprocessor-controlled prosthetic knee condition.

\section{Comparison of perturbed and non-perturbed walking}

Because we only found differences between prosthetic knee condition for the BMoS after the stance phase perturbation, we only compared the BMoS of the steps after the stance phase perturbation to non-perturbed walking. For the results of this comparison see Table 7.4. In the non-microprocessor-controlled prosthetic knee condition, the BMoS of the first step after the stance phase perturbation was significantly smaller when compared to nonperturbed walking. For the BMoS of the other steps, no differences were found. When looking at the variables that influence the BMoS, the anteroposterior center of mass velocity 
Table 7.3: Comparison of prosthetic knee conditions on variables that influence the backwards margin of stability

\begin{tabular}{lrcrrrrr}
\hline Variables & \multicolumn{2}{c}{ NMPK } & \multicolumn{2}{c}{ Rheo Knee II } & \multicolumn{2}{c}{ Median difference } & $P$ \\
\hline AP CoM vel 1 (m/s) & 0.71 & {$[0.62,0.73]$} & 0.69 & {$[0.67,0.86]$} & -0.46 & {$[-0.10,0.02]$} & 0.237 \\
AP CoM vel 2 (m/s) & 0.54 & {$[0.31,0.57]$} & 0.56 & {$[0.30,0.73]$} & -0.11 & {$[-0.16,0.05]$} & 0.499 \\
AP CoM vel 3 (m/s) & 0.62 & {$[0.48,0.73]$} & 0.72 & {$[0.57,0.81]$} & -0.06 & {$[-0.11,-0.01]$} & 0.028 \\
AP CoM vel 4 (m/s) & 0.57 & {$[0.47,0.68]$} & 0.61 & {$[0.42,0.72]$} & -0.03 & {$[-0.06,0.01]$} & 0.310 \\
AP CoM vel 5 (m/s) & 0.67 & {$[0.62,0.74]$} & 0.69 & {$[0.59,0.85]$} & -0.03 & {$[-0.08,0.02]$} & 0.176 \\
AP CoM vel 6 (m/s) & 0.62 & {$[0.43,0.70]$} & 0.65 & {$[0.37,0.73]$} & 0.02 & {$[0.01,0.03]$} & 0.735 \\
Step Length 1 (cm) & 51.12 & {$[44.09,56.18]$} & 53.82 & {$[45.37,56.74]$} & -2.20 & {$[-3.24,-0.564]$} & 0.237 \\
Step Length 2 (cm) & 46.06 & {$[24.79,52.79]$} & 34.94 & {$[28.56,51.91]$} & 1.37 & {$[-3.76,0.72]$} & 0.398 \\
Step Length 3 (cm) & 37.04 & {$[28.41,46.48]$} & 45.03 & {$[34.23,47.17]$} & -0.81 & {$[-0.58,1.89]$} & 0.398 \\
Step Length 4 (cm) & 41.84 & {$[35.28,54.24]$} & 36.14 & {$[34.71,43.13]$} & 1.85 & {$[0.57,11.11]$} & $\mathbf{0 . 0 1 8}$ \\
Step Length 5 (cm) & 36.57 & {$[36.19,45.99]$} & 42.88 & {$[35.42,45.94]$} & 0.22 & {$[-1.00,1.15]$} & 0.612 \\
Step Length 6 (cm) & 39.53 & {$[30.64,56.32]$} & 34.10 & {$[29.14,49.65]$} & 2.98 & {$[1.21,6.66]$} & $\mathbf{0 . 0 2 8}$ \\
FFP 1 (cm) & 15.25 & {$[11.48,18.85]$} & 14.58 & {$[11.44,16.90]$} & 0.73 & {$[0.40,1.15]$} & 0.176 \\
FFP 2 (cm) & 14.23 & {$[0.80,18.41]$} & 6.02 & {$[3.04,15.31]$} & 0.36 & {$[-1.91,4.73]$} & 0.398 \\
FFP 3 (cm) & 9.94 & {$[5.53,15.34]$} & 11.35 & {$[6.96,13.95]$} & 0.57 & {$[-1.41,2.99]$} & 0.499 \\
FFP 4 (cm) & 14.60 & {$[5.35,15.34]$} & 6.70 & {$[5.63,14.70]$} & 2.18 & {$[1.05,4.83]$} & $\mathbf{0 . 0 2 8}$ \\
FFP 5 (cm) & 13.97 & {$[7.85,15.34]$} & 12.43 & {$[7.22,13.99]$} & 1.35 & {$[0.29,1.73]$} & $\mathbf{0 . 0 1 8}$ \\
FFP 6 (cm) & 14.21 & {$[4.88,18.39]$} & 5.99 & {$[4.66,15.00]$} & 1.70 & {$[1.04,3.38]$} & $\mathbf{0 . 0 1 8}$ \\
\hline Data are presented as & & & & &
\end{tabular}

Data are presented as median [interquartile range]. The median difference is calculated by subtracting the value of Rheo Knee II condition from the NMPK condition.

Abbreviations: NMPK: non-microprocessor-controlled prosthetic knee; BMoS: backward margin of stability; AP CoM vel: anteroposterior center of mass velocity; FFP: foot forward placement

at initial contact of step 1 was significantly increased and velocity at initial contact of step 2 was significantly decreased when compared to non-perturbed walking. The step length of the intact leg was both statistically significantly increased (step 1) and decreased (step 3 and 5) when compared to non-perturbed walking. The step length of the prosthetic leg was significantly increased for all steps (step 2, 4, and 6) when compared to non-perturbed walking. The foot forward placement was significantly decreased for step 1 (intact leg) when compared to non-perturbed walking.

For the Rheo Knee II condition, the BMoS of step 3 through 6 (resp. intact, prosthetic, intact, prosthetic leg) was significantly increased when compared to non-perturbed walking. The anteroposterior center of mass velocity showed the same pattern as the nonmicroprocessor-controlled prosthetic knee: significantly increased at step 1 (intact leg) and significantly decreased at step 2 (prosthetic leg) when compared to non-perturbed walking. Step length and foot forward placement showed similar patterns: a significantly increased value for step 1 (intact leg) and a significantly decreased value for step 2 through 6.

For the individuals without an amputation, the BMoS of step 2 through 6 was significantly increased when compared to non-perturbed walking. The general pattern after the perturbation was to decrease anteroposterior center of mass velocity and step length and foot forward placement. 

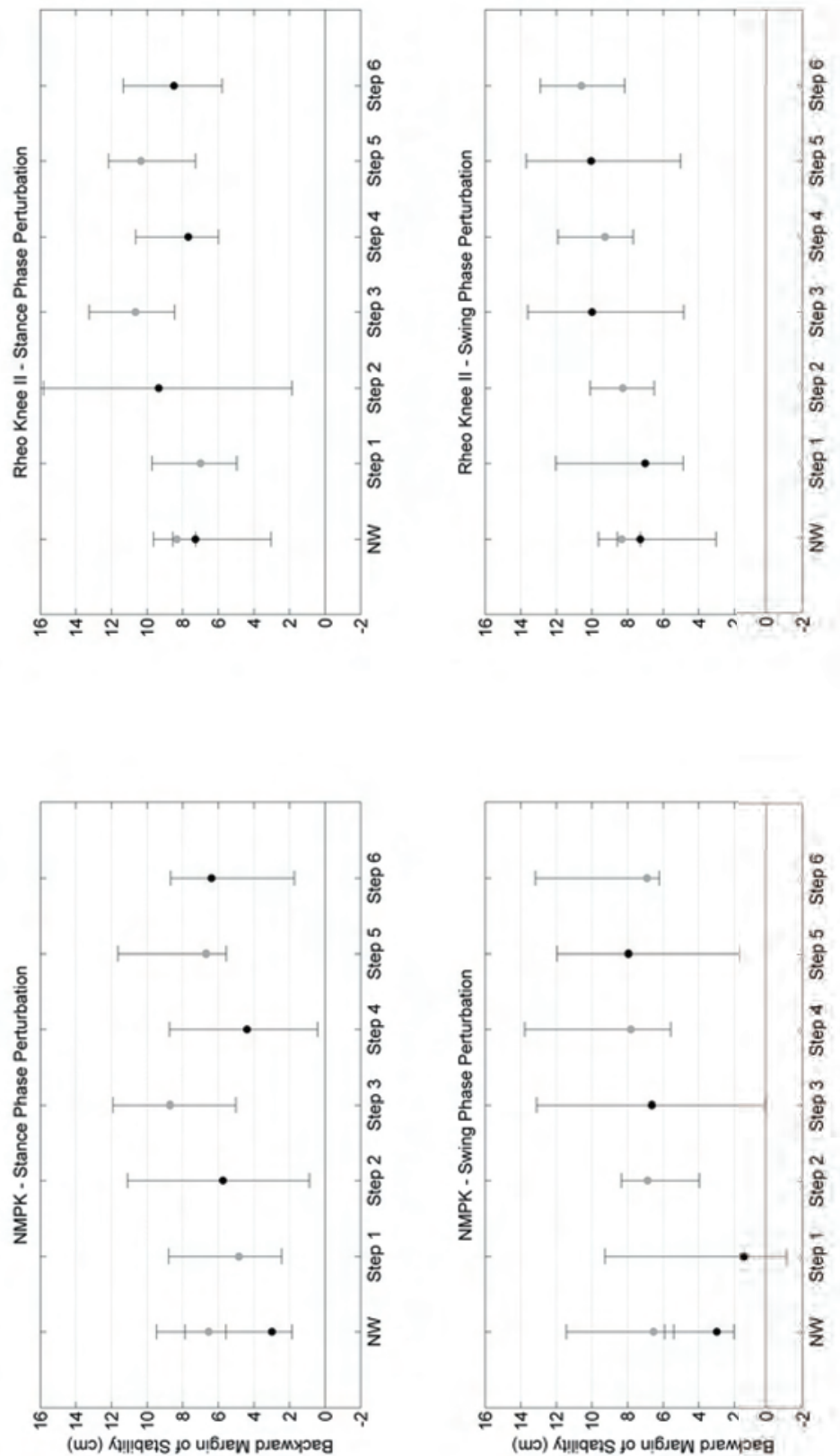

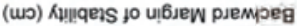

Figure 7.2: Graphical representation of the Backward Margin of Stability.

The dots represent the median value and the error bars represent the interquartile range. The dots in black represent the prosthetic leg and the dots in gray represent the intact leg.

Abbreviations: NMPK: non-microprocessor-controlled prosthetic knee; NW: non-perturbed walking. 


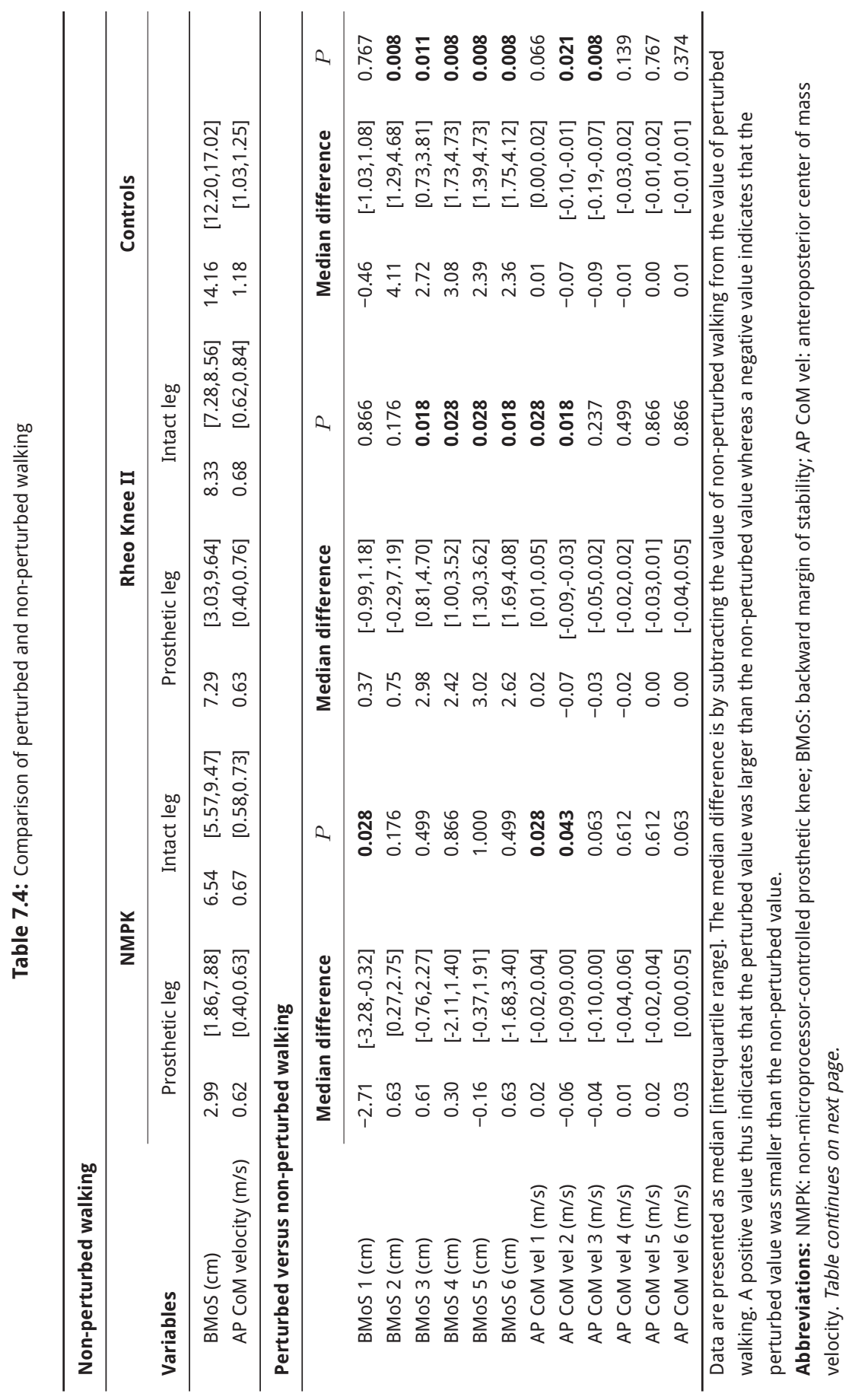




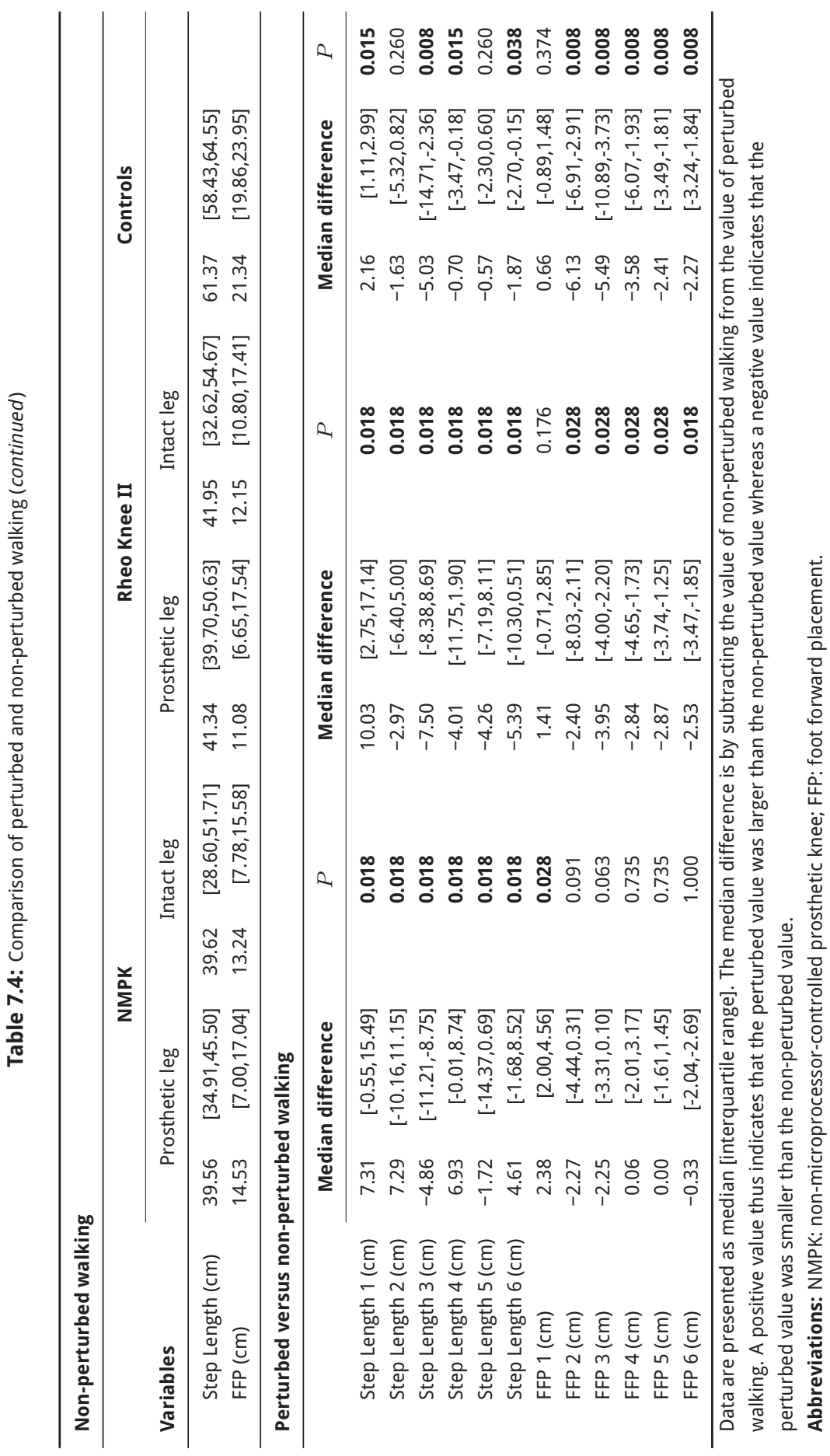




\section{Discussion}

The aim of this study was to compare the response of individuals with a transfemoral amputation or knee disarticulation to anteroposterior stance and swing phase perturbations of the prosthetic leg during walking while wearing a non-microprocessor-controlled prosthetic knee and the Rheo Knee II. To do so, we compared the two prosthetic knee conditions with one another and we compared perturbed to non-perturbed walking. Because the use of microprocessor-controlled prosthetic knees is attributed with increased stance phase stability, we hypothesized increased BMoS and ML-MoS while using the Rheo Knee II when compared to the use of non-microprocessor-controlled prosthetic knees. In addition, we hypothesized that individuals were able to use similar strategies as individuals without an amputation to cope with balance perturbations when walking with the Rheo Knee II.

Comparison of the BMoS of the six steps after the stance phase perturbation showed statistically significantly higher values in the Rheo Knee II condition when compared to the non-microprocessor-controlled prosthetic knee condition, suggesting increased stability and thus a decreased fall risk in the Rheo Knee II condition after stance phase perturbations. When looking at the origin of the difference between prosthetic knees, one can distinguish step 1 from step 2 through 6 . The difference in BMoS at initial contact of step 1 between the prosthetic knees is the result of a decreased BMoS in the non-microprocessor-controlled prosthetic knee condition, as opposed to an increased BMoS in the Rheo Knee II condition in steps 2-6. Looking at the variables that influence the BMoS show that the reduced BMoS in the non-microprocessor-controlled prosthetic knee condition is mainly caused by an increased step length and foot forward placement.Studying the variables that influence the BMoS shows that the strategy to increase the BMoS in the Rheo Knee II condition is mainly to slightly increase CoM velocity and decrease the foot forward placement when compared to the non-microprocessor-controlled prosthetic knee condition (see Table 7.3). In summary, although the differences on the secondary outcome parameters were mostly non-significant, all variables combined did lead to significant differences in BMoS between the prosthetic knee conditions.

Comparison of the steps after the stance phase perturbation to non-perturbed walking showed that the use of the Rheo Knee II enabled the use of strategies that were also used by individuals without an amputation. This was not the case for the non-microprocessorcontrolled prosthetic knee condition. For the Rheo Knee II condition the BMoS of step 3 through 6 were significantly higher when compared to non-perturbed walking where this was the case for step 2 through 6 for individuals without an amputation. In the nonmicroprocessor-controlled prosthetic knee condition the BMoS of step 1 was significantly decreased when compared to non-perturbed walking where no differences for the other steps were seen. When looking at the variables that influence the BMoS striking similarities were seen between the Rheo Knee II condition and the control group. Although not all differences were statistically significant, the general pattern was to slightly decrease antero- 
posterior center of mass velocity and decrease step length and foot forward placement of the steps after the perturbation when compared to non-perturbed walking.

In the non-microprocessor-controlled prosthetic knee condition, a distinctly different strategy was used to cope with the balance perturbations. In the non-microprocessorcontrolled prosthetic knee condition, the BMoS of the first step (intact leg) after the stance phase perturbation was significantly smaller when compared to non-perturbed walking. This, was caused by an increased step length and foot forward placement. The BMoS of step 2 through 6 after the stance phase perturbation were comparable to the BMoS of nonperturbed walking. Although differences in anteroposterior center of mass velocity, step length, and foot forward placement were visible between non-perturbed and perturbed walking in the non-microprocessor-controlled prosthetic knee condition, the interplay between these variables led to a comparable BMoS of step 2 through 6 . Most importantly, an increased center of mass velocity was accompanied with a larger foot forward placement and a decreased center of mass velocity was accompanied with a smaller foot forward placement. The important question is why the Rheo Knee II enabled the use of strategies that were also used by the controls whereas in the non-microprocessor-controlled prosthetic knee condition this was not the case. A possible explanation for this could be the non-optimal knee damping during early stance that is associated with the use of nonmicroprocessor-controlled prosthetic knees. These knees have to be extended at the beginning of the stance phase, else they might buckle and the individual with an amputation might fall. Achieving full knee extension at the beginning of the stance phase usually requires a large step length. The Rheo Knee II should provide increased early stance stability which might allow a smaller step length without an increased risk of knee buckling.

In contrast to our hypothesis we did not find differences for the swing phase perturbations. One possible explanation for this is that the timing of the perturbations was relatively late: around $90 \%$ of the gait cycle. We hypothesized a potential advantage for the Rheo Knee II when participants had to start the stance phase with non-fully extended prosthetic knee. Applying the perturbations around $90 \%$ of the gait cycle probably enabled the participants to fully extend their prosthetic knee before the beginning of the stance phase, thereby reducing the advantage that the Rheo Knee II might have. In addition, we did not find differences in ML-MoS between prosthetic knee conditions. Possible explanations for this finding includes that fact that perturbations were applied in the anteroposterior direction and the fact that both prosthetic knees only act in the sagittal plane and do not allow any movement in frontal plane.

The fact that individuals with an amputation are able to use similar strategies as individuals without an amputation were also found by other authors. Hak et al. studied the effect of continuous mediolateral balance perturbations on the gait of individuals with a transtibial amputation and without an amputation. ${ }^{168}$ They found the balance perturbation led to a decrease in step length, increase in step frequency and increased backward margin of stability when compared to non-perturbed walking. ${ }^{168}$ These results are in line with the 
results we found for the Rheo Knee II condition and the individuals without an amputation. Shirota et al. ${ }^{164}$ perturbed the swing phase of both individuals with a transfemoral amputation or knee disarticulation and without an amputation. They found that both groups use similar strategies although specific strategies are used more often in one group when compared to the other. ${ }^{164}$ This study was confounded by a number of factors. First of all we had a limited sample size, which affected the statistical power of the study. However, we were able to detect statistical significant differences between prosthetic knee conditions. It remains to be seen whether these differences hold up in a properly powered study and/or non-significant differences become significant. Next to this, we had a study population with a high functional level and none of the participants had an amputation due to vascular problems, which is the main reason for amputation. The results of this study can therefore only be generalized to a small proportion of the overall population of individuals with an amputation. Thirdly, we were not able to obtain kinetic variables because we used a singlebelt instrumented treadmill. Studying kinetic variables could have assisted us in providing a better explanation as to why differences between prosthetic knees existed. Finally, we chose to perform the experiments with an individually determined fixed treadmill speed. During preliminary trials we noticed that platform perturbations in combination with a selfpaced treadmill speed could provide an even bigger challenge to stability (for example, the participants that briefly stopped walking after the perturbation had additional balance perturbations because the treadmill suddenly slowed down). The fact that treadmill speed was fixed could have limited the participants in the way they coped with balance perturbations. For example, participants that wished to increase their walking speed after the balance perturbations were limitedly able to do so, because they would have walked off the treadmill by doing so.

Future research could focus on increasing the study populations to validate the findings of our study. In addition, they could use perturbations with varying magnitude to investigate whether there are other strategies to cope with balance perturbations than the ones we have described and whether the choice of strategy is dependent on perturbation magnitude. Finally, future studies could measure kinetic variables of the response to balance perturbations to provide a better understanding as to why differences between prosthetic knees exist.

In conclusion, walking with the Rheo Knee II enabled participants to use strategies, also usedby non-amputees to cope with the balance perturbations. The increased BMoS in the Rheo Knee II condition, is suggestive of a decreased fall risk after the stance phase perturbations when compared to the non-microprocessor-controlled prosthetic knee condition. No differences were visible after swing phase perturbations. This study provides the first biomechanical findings that might explain the earlier reported decreased fall risk associated with the use of microprocessor-controlled prosthetic knees. 



\section{Influence of a user-adaptive}

prosthetic knee on quality of life, balance confidence and measures of mobility: a randomized cross-over trial

Prinsen EC, Nederhand MJ, Olsman J, Rietman JS. Clin Rehab 2015;29:581-91. 


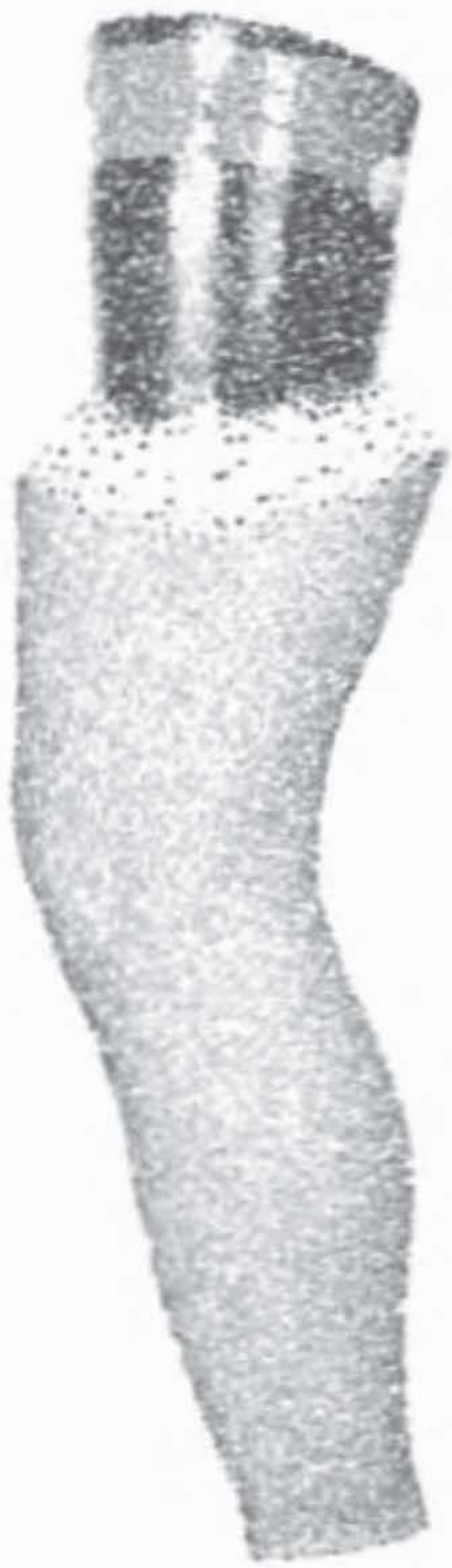




\section{Abstract}

Objective To study the influence of a transition from a non-microprocessor-controlled prosthetic knee to the Rheo Knee II on quality of life, balance confidence and measures of mobility.

Design Randomized crossover trial.

Setting Research department of a rehabilitation centre.

Subjects Persons with a transfemoral amputation or knee disarticulation $(n=10)$.

Interventions Participants were assessed with their own non-microprocessor-controlled knee and with the Rheo Knee II. The low-profile Vari-Flex ${ }^{\circledR}$ with EVO ${ }^{\mathrm{TM}}$ foot was installed in both knee conditions, followed by eight weeks of acclimatisation. The order in which knees were tested was randomized.

Main Measures Prosthesis Evaluation Questionnaire with addendum, Activities-specific Balance Confidence scale, Timed "up \& go" Test, Timed up and down stairs test, Hill Assessment Index, Stairs Assessment Index, Standardized Walking Obstacle Course and One Leg Balance test.

Results Significant higher scores were found for the Rheo Knee II on the Residual Limb Health subscale of the Prosthesis Evaluation Questionnaire when compared to the nonmicro-processor-controlled prosthetic knee (median [interquartile range] respectively 86.67 [62.21-93.08] and 68.71 [46.15-94.83]; $P=0.047$ ) In addition, participants needed significantly more steps to complete an obstacle course when walking with the Rheo Knee II compared to the non-microprocessor-controlled prosthetic knee (median [interquartile range] respectively 23.50 [19.92-26.25] and 22.17 [19.50-25.75]; $P=0.041$ ). On other outcome measures, no significant differences were found.

Conclusions Transition towards the Rheo Knee II had little effect on the studied outcome measures. 


\section{INTRODUCTION}

In persons with a transfemoral amputation or knee disarticulation, different types of prosthetic knees are available. These include non-microprocessor-controlled and microprocessor-controlled prosthetic knees. In contrast to non-microprocessor-controlled prosthetic knees, microprocessor controlled prosthetic knees are capable of adjusting the damping properties to changing user and/or environmental features (e.g. walking speed, prosthetic loading, stairs or ramps). ${ }^{7,20}$ This may result in a more natural and stable gait. Several studies investigated whether a transition from a non-microprocessor-controlled to a microprocessor-controlled prosthetic knee, such as the C-Leg or Rheo Knee, improved aspects of daily living.

Studies investigating the influence of the C-Leg compared to non-microprocessor-controlled prosthetic knees on quality of life show ambiguous results on the Prosthesis Evaluation Questionnaire. ${ }^{26,27,32,41,170}$ On performance based measures of mobility, studies reported increased walking speed on both even and uneven terrain, ${ }^{26}$ decrease in time that was needed to complete the Timed "up \& go" Test, ${ }^{171}$ increased performance of ramp and stair de-scent, ${ }^{27,32,36}$ and improved obstacle course navigation when walking with a microprocessor-controlled prosthetic knee. ${ }^{33,35}$ Finally, studies found no differences in daily step count between prosthetic knee conditions. ${ }^{27,145}$

Previous research mainly focused on the C-Leg. Scientific research focusing on the Rheo Knee is scarcely available. ${ }^{11,63}$ Because the control algorithm of the Rheo Knee II is useradaptive and the control algorithm of the C-Leg is not, both knees might have a different impact on daily functioning. Furthermore, the majority of authors have used a pretest-posttest design. While this is consistent with clinical practice, it has limitations such as the possibility of introducing a learning effect. Only few studies randomized the order in which prosthetic knees were tested. ${ }^{12,35,145}$ Accordingly, there is limited evidence from randomized studies to help guide clinical decision making. Therefore, we aim to study the influence of a transition from a non-microprocessor-controlled prosthetic knee towards the user-adaptive Rheo Knee II on aspects of daily functioning using a randomized within subject cross-over trial.

\section{Methods}

\section{Participants}

For this randomized within subject crossover trial participants with a transfemoral amputation or a knee disarticulation were recruited from a convenience sample in the Netherlands and Belgium. The inclusion criteria were: (1) at least one year post amputation, (2) functional level from K2 (limited mobility) to K4 (high activity) ${ }^{142}$ as assessed by treating physiatrist, (3) never supplied with a microprocessor-controlled knee unit before. Exclusion criteria were: (1) other musculoskeletal problems influencing walking ability, (2) stump 
problems/bad socket fitting, (3) body weight $>125 \mathrm{Kg}$ (maximum tolerable weight of the Rheo Knee II), (4) knee center-floor distance insufficient to allow fitting of the Rheo Knee II. The study protocol was approved by the local medical ethical research committee and all subjects provided written informed consent before inclusion.

\section{Protocol}

Participants were randomly assigned to start measurements with their own non-microprocessor controlled prosthetic knee or to start with the Rheo Knee II. We used a 4 block randomization procedure to ensure comparable group sizes. In both prosthetic knee conditions the LP Vari-Flex ${ }^{\circledR}$ with $\mathrm{EVO}^{\mathrm{Tm}}$ (Össur) was used, thereby excluding the effect of prosthetic foot on outcome measures. After inclusion participants had an appointment with the prosthetist to install either the LP Vari-Flex with EVO or the Rheo Knee II and the LP Vari-Flex with EVO. After eight weeks of acclimatization the first measurements took place after which subjects crossed over to the other prosthetic condition. After another eight weeks, the second set of measurements was performed. After measurements were finalized, the prosthesis was converted to the original state and subjects left the study. Alignment of the non-microprocessor-controlled prosthetic knee was carefully checked before any adjustments were made during the first appointment with the prosthetist. In case the alignment was not according to manufacturer's guidelines, it was changed accordingly. The alignment of the non-microprocessor-controlled prosthetic knee was carefully transferred to the Rheo Knee II condition, to ensure comparable alignment. In case this resulted in a load line outside the manufacturer's recommendation for the Rheo Knee II, alignment was adjusted accordingly. All prosthetic adjustments were performed by the same prosthetist. Participants did not receive a gait training program while walking with the Rheo Knee II. Our main consideration in making this choice was that we aimed to make the comparison as unaffected by gait training factors as possible.

\section{Outcome measures}

The Prosthetic Evaluation Questionnaire is a questionnaire aimed at measuring prostheticrelated quality of life in amputees. ${ }^{172}$ It consists of nine validated subscales (utility, residual limb health, appearance, sounds, ambulation, perceived responses, social burden, frustration, and well-being). Hafner et al. ${ }^{27}$ added an addendum consisting of 14 questions regarding subject confidence, concentration, and falls. The questions comprising a subscale were averaged. The questions of the addendum were analysed separately. The Prosthetic Evaluation Questionnaire is validated for unilateral amputees, one year post-amputation who wear their prosthesis at least five days a week. ${ }^{172}$ The Prosthesis Evaluation Questionnaire showed fair-to-strong reliability. ${ }^{172,173}$ The psychometric properties of the addendum are, so far, unknown.

The Activities-specific Balance Confidence scale is a 16 item questionnaire measuring the balance confidence of an individual completing different ambulatory activities. ${ }^{174}$ This scale 
has been validated using prosthetically and medically stable persons with a transfemoral amputation using their prosthesis at least three times a week. ${ }^{175}$ Internal consistency and reliability were found to be strong and good support for construct and discriminant ability was found. ${ }^{175}$ Translation of the Activities-specific Balance Confidence scale in Dutch was done by Vrieling et al. ${ }^{176}$. Cross-translation resulted in the original questionnaire.

The Timed "up and go" Test measures the time a subjects needs to get up from a chair, walk three meters, turn around, walk three meters back, and sit down on a chair. ${ }^{177}$ Measurements were stopped when three trials were available which were averaged. The Timed "up and go" Test has proven to be a reliable instrument for unilateral amputees, showing good inter- and intrarater reliability. ${ }^{178}$

The Timed Up and Down Stairs test measures the time a subject needs to quickly, but safely, go up the stairs, turn around on the top step and come all the way down until both feet land on the bottom step. ${ }^{179}$ The starting position was standardized by positioning the subject $30 \mathrm{~cm}$ in front of the bottom step. Three trials were completed and scores were averaged. The psychometric properties of the Timed Up and Down Stairs test for transfemoral amputees are unknown.

The Stair Assessment Index is a 14-level instrument to score the stair ascent and descent ability. ${ }^{180}$ Possible scores range from 0 to 13 . The stairs that were used had steps $25 \mathrm{~cm}$ deep and $16.5 \mathrm{~cm}$ high and had a rise percentage of $66 \%$. The stairs were $1 \mathrm{~m}$ wide with handrails on both sides.

The Hill Assessment Index is a 12-level instrument to score the independence and technique that is used during hill descent. ${ }^{181}$ Possible scores range from 0 to 12 . The Hill Assessment Index was administered on a CAREN platform at the National Military Rehabilitation Centre Aardenburg in Doorn, the Netherlands. The CAREN platform incorporates a treadmill which was set at preferred walking speed. Because we used a fixed treadmill speed, we considered the time to complete the Hill Assessment Index not useful as outcome measure. Therefore we chose to present the treadmill speed next to the Hill Assessment Index score.

The Standardized Walking Obstacle Course consists of a $12.2 \mathrm{~m}$ walkway that includes a low profile rug with a $30^{\circ}$ turn to the right, a $90^{\circ}$ turn to the left, and then a $70^{\circ}$ turn to the right. ${ }^{182}$ The obstacle course starts with rising of a chair with armrests, step over an elbow crutch, walk over a visually challenging rug, maneuver around a trashcan, walk over a shag rug, and sit down on a chair without armrests. The measures within the Standardized Walking Obstacle Course are time, number of steps, number of step-offs and number of stumbles. Three trials were completed. The scores on the three trials were averaged.

The One Legged Balance Test times the ability of a subject to stand on one leg. At the start of the test, subjects stood on both legs with eyes open. After the researcher counted backwards from three to one, the subject was expected to raise one leg and maintain balance. The test was terminated when the non-supporting leg touched the ground or balance was maintained for 30 seconds, which is the ceiling of the test. ${ }^{183}$ Three trials were completed for both leg conditions after which the scores were averaged. 
The person collecting the data and making the observations was not blinded for prosthetic condition.

\section{Data analysis}

Data were analyzed using IBM SPSS Statistics 19.0 (IBM SPSS Statistics, Chicago, USA). Because of the small sample size, non-parametric tests were chosen. The first step of the statistical analysis was to check whether the order in which the prosthetic knees were tested affected the results (a so-called order effect). The scores of the Rheo Knee II were subtracted from the scores of the non-microprocessor-controlled prosthetic knee. This difference score was than tested with a Mann-Whitney $U$ test to check for significant differences between the group that started with the Rheo Knee II and the group that started with the non-microprocessor-controlled prosthetic knee.

In case of a non-significant order effect all data could be combined. In this case a Wilcoxon Signed Rank Sum test was used to test for significant differences between prosthetic knees. In case of a significant order effect, the data could not be combined for all subjects, as the initial prosthetic knee condition confounded the results of the other prosthetic knee condition. Therefore only the data of the first measurement could be analyzed. This means that data of the Rheo Knee II collected in the group that started with the Rheo Knee II was compared to data of the non-microprocessor-controlled prosthetic knee that was collected in the group that started with the non-microprocessor-controlled prosthetic knee. In this instance, a Mann-Whitney $U$ test was used to check for significant differences between prosthetic knees.

Statistical significance was set at $P \leq 0.05$ for all comparisons.

\section{RESULTS}

The result of the recruitment process is displayed in Figure 8.1. Descriptive variables of the participants are displayed in Table 8.1. 


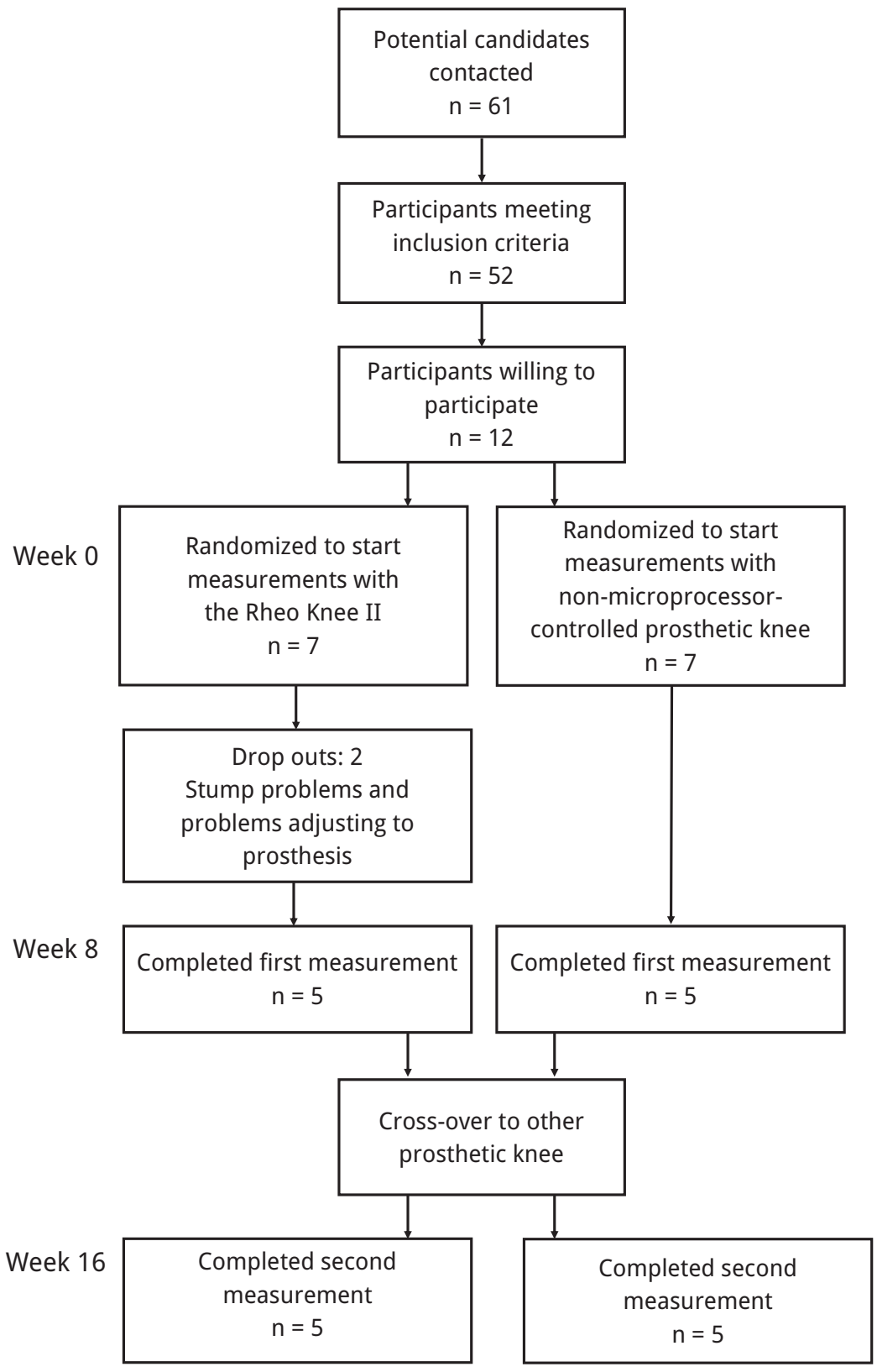

Figure 8.1: Flow diagram recruitment 
Table 8.1: Descriptive variables participants

\begin{tabular}{ll}
\hline Variables & Participants ( $\mathbf{= 1 0})$ \\
\hline Age (years) & $53(23-67)$ \\
Sex (male/female) & $6 / 4$ \\
Time since amputation (years) & $33.5(1-41)$ \\
Reason for amputation & Trauma (7), Infection (2), Osteosarcome (1) \\
Functional level & K2 (2), K3 (5), K4 (3) \\
Stump length (cm) & $40(32-60)$ \\
Non-microprocessor-controlled prosthetic knees & $3 R 60(4), 3 R 80(1)$, Mauch SNS (1), Graph \\
& Lite (1), CaTech (1), Total Knee 2000 (1) \\
\hline
\end{tabular}

Age, time since amputation, and stump length are presented as median (range).

Sex, reason for amputation, functional level, and non-microprocessor-controlled prosthetic

knees are presented as counts

\section{Prosthesis-related quality of life and balance confidence}

Results of prosthesis-related quality of life and balance confidence are displayed in Table 8.2. The first step of the data analysis was to check for significant order effects. This was found for the sounds subscale of the Prosthesis Evaluation Questionnaire. For the analysis of this subscale we thus compared the data collected in the first measurement. No order effects were found for the other subscales/items of the Prosthesis Evaluation Questionnaire, addendum, or Activities-specific Balance Confidence scale.

Results of the Prosthesis Evaluation Questionnaire show a significant higher score for the Rheo Knee II on the Residual Limb Health subscale when compared to the non-microprocessor controlled prosthetic knee (median [interquartile range] resp. 86.67 [62.21-93.08] and 68.71 [46.15-94.83]; $P=0.047$ ).

The other eight subscales show no significant differences. In addition, no clear trend is visible when the median and interquartile range scores are visually inspected. Results of the Prosthesis Evaluation Questionnaire addendum and the Activities-specific Balance Confidence scale showed no significant differences or clear trends.

\section{Measures of mobility}

Results of the performance based measures of mobility are displayed in Table 8.3. No significant order effects were found. The results indicate that significantly more steps were needed to execute the Standardized Walking Obstacle Course while walking with the Rheo Knee II when compared to the non-microprocessor-controlled prosthetic knee (median [interquartile range] resp. 23.50 [19.92- 26.25] and 22.17 [19.50-25.75]; $P=0.041$ ). On the other tests no significant differences were found.

\section{Post-hoc analysis}

To test the robustness of the results on the Sounds subscale, we performed a post-hoc analysis in which the order effect was ignored. This analysis showed a significant improve- 
ment on the Sounds subscale in favor of the Rheo Knee II when compared to the nonmicroprocessor-controlled prosthetic knee (median [interquartile range] resp. 85.00 [61.8896.75] and 50.25 [12.25-62.88]; $P=0.022)$.

Table 8.2: Results of the prosthesis-related quality of life and balance confidence

\begin{tabular}{|c|c|c|c|c|c|c|c|}
\hline $\begin{array}{l}\text { Outcome measures } \\
\text { PEQ }\end{array}$ & \multicolumn{2}{|c|}{ Score NMPK } & \multicolumn{2}{|c|}{ Score Rheo Knee II } & $P$ & Z score & ES \\
\hline Ambulation & 64.38 & [45.88-76.32] & 68.25 & [48.35-81.81] & 0.333 & -0.968 & 0.22 \\
\hline Appearance & 67.20 & {$[47.94-80.06]$} & 65.13 & [54.13-73.91] & 0.878 & 1.530 & 0.34 \\
\hline Frustration & 77.25 & [33.38-97.88] & 61.00 & {$[28.63-90.75]$} & 0.594 & -0.533 & 0.12 \\
\hline Perceived Response & 87.47 & [61.80-95.85] & 81.20 & [69.53-93.25] & 0.878 & -0.153 & 0.03 \\
\hline Residual Limb Health & 68.71 & [46.15-94.83] & 86.67 & [62.21-93.08] & 0.047 & -1.988 & 0.44 \\
\hline Social Burden & 95.67 & [24.33-97.50] & 95.50 & {$[65.50-96.88]$} & 0.760 & -0.306 & 0.07 \\
\hline Sounds* & 59.50 & {$[50.25-80.00]$} & 93.50 & [63.25-97.50] & 0.347 & -0.940 & 0.30 \\
\hline Utility & 67.69 & [52.47-90.53] & 78.07 & [62.81-85.44] & 0.333 & -0.968 & 0.22 \\
\hline Well-being & 71.00 & [54.00-90.38] & 77.75 & [51.38-90.00] & 0.683 & -0.408 & 0.09 \\
\hline \multicolumn{8}{|l|}{ PEQ addendum } \\
\hline $\begin{array}{l}\text { Mental energy } \\
\text { expenditure }\end{array}$ & 68.50 & [30.50-93.25] & 81.50 & [39.25-93.00] & 0.799 & -0.255 & 0.06 \\
\hline $\begin{array}{l}\text { Frequency of } \\
\text { stumbling }\end{array}$ & 51.50 & {$[34.25-85.00]$} & 64.50 & {$[35.00-84.00]$} & 0.799 & -0.255 & 0.06 \\
\hline Number of stumbles & 4.00 & {$[0.50-6.00]$} & 5.00 & [1.50-22.00] & 0.214 & -1.244 & 0.28 \\
\hline $\begin{array}{l}\text { Frequency of } \\
\text { semi-controlled falling }\end{array}$ & 82.50 & [58.25-98.25] & 79.50 & {$[55.75-95.25]$} & 0.919 & -0.102 & 0.02 \\
\hline $\begin{array}{l}\text { Number of } \\
\text { semi-controlled falls }\end{array}$ & 2.50 & {$[0.00-12.50]$} & 2.50 & [0.00-16.25] & 0.715 & -0.365 & 0.08 \\
\hline $\begin{array}{l}\text { Frequency of } \\
\text { uncontrolled falling }\end{array}$ & 96.50 & [82.50-99.25] & 90.50 & {$[55.75-98.75]$} & 0.673 & -0.422 & 0.09 \\
\hline $\begin{array}{l}\text { Number of } \\
\text { uncontrolled falls }\end{array}$ & 0.00 & {$[0.00-1.75]$} & 0.00 & {$[0.00-4.25]$} & 0.450 & -0.756 & 0.17 \\
\hline $\begin{array}{l}\text { Confidence while } \\
\text { walking }\end{array}$ & 86.50 & {$[61.75-96.25]$} & 82.00 & {$[60.50-90.25]$} & 0.359 & -0.918 & 0.21 \\
\hline $\begin{array}{l}\text { Difficulty multitasking } \\
\text { while walking }\end{array}$ & 82.00 & {$[58.75-97.25]$} & 81.00 & {$[53.00-97.00]$} & 0.635 & -0.474 & 0.11 \\
\hline Fear of falling & 95.50 & [38.25-98.25] & 88.00 & {$[56.25-95.00]$} & 0.677 & -0.416 & 0.09 \\
\hline Frustration with falling & 92.50 & {$[66.25-97.25]$} & 88.50 & {$[46.50-95.75]$} & 0.575 & -0.561 & 0.13 \\
\hline $\begin{array}{l}\text { Embarrassment with } \\
\text { falling }\end{array}$ & 90.50 & [54.25-97.25] & 86.50 & {$[68.00-94.25]$} & 0.953 & -0.059 & 0.01 \\
\hline $\begin{array}{l}\text { Fearful of falling } \\
\text { without the prosthesis }\end{array}$ & 88.00 & {$[52.50-97.25]$} & 86.50 & {$[41.50-93.50]$} & 0.575 & -0.560 & 0.13 \\
\hline $\begin{array}{l}\text { Difficulty with } \\
\text { concentration }\end{array}$ & 77.00 & {$[28.75-96.50]$} & 86.50 & {$[35-25-94.75]$} & 0.878 & -0.153 & 0.03 \\
\hline ABC-scale & 71.08 & {$[44.60-90.16]$} & 74.50 & [57.43-90.69] & 0.575 & -0.561 & 0.13 \\
\hline
\end{tabular}

*Significant order effect: score NMPK are of group that started measurements with NMPK; scores Rheo Knee II are of group that started with the Rheo Knee II. Scores are presented as median [interquartile range]

Abbreviations: NMPK: non-microprocessor-controlled prosthetic knee; ES: effect size; PEQ: Prosthesis Evaluation Questionnaire; ABC: Activities-specific Balance Confidence 
Table 8.3: Results of the measures of mobility

\begin{tabular}{lrrrrrrr}
\hline Outcome measures & \multicolumn{2}{c}{ Score NMPK } & \multicolumn{2}{c}{ Score Rheo Knee II } & $P$ & Z score & ES \\
\hline TUGT (sec) & 10.52 & {$[9.45-11.13]$} & 10.53 & {$[9.65-10.83]$} & 0.959 & -0.051 & 0.01 \\
TUDS (sec) & 22.63 & {$[14.55-24.60]$} & 22.66 & {$[13.81-26.85]$} & 0.508 & -0.663 & 0.15 \\
SAI & & & & & & & \\
$\quad$ Score stair ascent & 3.00 & {$[3.00-3.00]$} & 3.00 & {$[3.00-3.00]$} & 1.000 & 0.000 & 0.00 \\
$\quad$ Score stair descent & 3.00 & {$[3.00-3.00]$} & 3.00 & {$[3.00-3.00]$} & 1.000 & 0.000 & 0.00 \\
HAI & & & & & & & \\
$\quad$ Score & 8.00 & {$[8.00-8.00]$} & 8.00 & {$[8.00-8.25]$} & 0.317 & 1.000 & 0.22 \\
$\quad$ Speed (m/s) & 0.70 & {$[0.65-0.76]$} & 0.70 & {$[0.68-0.80]$} & 0.726 & -0.351 & 0.11 \\
SWOC & & & & & & & \\
$\quad$ Time (sec) & 18.29 & {$[16.11-19.64]$} & 18.02 & {$[16.42-21.72]$} & 0.508 & -0.663 & 0.15 \\
$\quad$ Steps (count) & 22.17 & {$[19.50-25.75]$} & 23.50 & {$[19.92-26.25]$} & 0.041 & -2.043 & 0.46 \\
$\quad$ Step-offs (count) & 0.00 & {$[0.00-0.00]$} & 0.00 & {$[0.00-0.00]$} & 1.000 & 0.000 & 0.00 \\
$\quad$ Stumbles (count) & 0.00 & {$[0.00-0.00]$} & 0.00 & {$[0.00-0.00]$} & 1.000 & 0.000 & 0.00 \\
OLBT & & & & & & & \\
$\quad$ Intact leg (sec) & 30.00 & {$[27.51-30.00]$} & 30.00 & {$[29.28-30.00]$} & 0.180 & -1.340 & 0.30 \\
$\quad$ Prosthetic leg (sec) & 1.15 & {$[0.19-1.73]$} & 1.15 & {$[0.20-2.34]$} & 0.779 & -0.280 & 0.06 \\
\hline
\end{tabular}

Data are presented as median [interquartile range].

Abbreviations: NMPK: non-microprocessor-controlled prosthetic knee; ES: effect size; TUGT: Timed "up and go" Test; TUDS: Timed Up and Down Stairs test; SAI: Stair Assessment Index; HAI: Hill Assessment Index; SWOC: Standardized Walking Obstacle Course; OLBT: One Legged Balance Test.

\section{DISCUSSION}

In this study, we found that the added value of the Rheo Knee II on prosthesis-related quality of life, balance confidence, and measures of mobility is limited compared to a nonmicroprocessor-controlled prosthetic knee.

Results of the Prosthesis Evaluation Questionnaire showed a significant increase on the Residual Limb Health subscale of the Prosthesis Evaluation Questionnaire while walking with the Rheo Knee II when compared to the non-microprocessor-controlled prosthetic knee. This subscale encompasses questions regarding swelling and pain of the stump, as well as questions asking whether rashes, ingrown hairs, or blisters are present on the stump. The found significant difference could be of clinical importance, as skin problems of the stump have shown to interfere with functioning in daily life. ${ }^{184}$ However, the increase in residual limb health did not translate to changes on other subscales of the Prosthesis Evaluation Questionnaire, thereby leaving the clinical importance of this finding questionable. Our findings are in line with earlier reported findings. Some trials report no differences, ${ }^{27,32}$ whereas others do find differences on variable subscales of the Prosthesis Evaluation Questionnaire. ${ }^{26,41,170}$ All in all, there seems to be no univocal evidence for a positive effect of microprocessor-controlled prosthetic knees on prosthesis-related quality of life. 
On the Sounds subscale of the Prosthesis Evaluation Questionnaire an order effect was present. Because of the order effect, groups were no longer comparable after the first measurement. Therefore, we only could incorporate data obtained during the first measurement in the data analysis. While this procedure is statistically more sound, it cut our sample size in half when compared to the other analyses (respectively 5 versus 5 instead of 10 versus 10), which reduced statistical power. To test the robustness of this finding, we performed a post-hoc analysis in which the order effect was ignored. This analysis found a significant increase in favour of the Rheo Knee II. Future research should elucidate what the influence of a transition towards the Rheo Knee II on the Sounds subscale of the Prosthesis Evaluation Questionnaire is.

On balance confidence we did not find differences. This is somewhat surprising, as we thought that balance confidence would increase because it is thought that microprocessorcontrolled prosthetic knees enable more stable gait. However, this hypothesis has not been confirmed yet in dynamic situations. It is therefore hard to speculate as to why we did not find differences. As far as we know, this is the first time that a validated questionnaire regarding balance confidence was administered in the comparison of microprocessorcontrolled and non-microprocessor-controlled prosthetic knees. Therefore a comparison to previously conducted studies was not possible.

On measures of mobility we found a significant increase in the number of steps needed to navigate the obstacle course when walking with the Rheo Knee II. While differences were small, they were consistent amongst participants. However, we believe that the difference of one step is not clinically relevant. This finding is in contrast with findings in previously conducted trials who found a decrease in needed number of steps to navigate an obstacle course. $^{33}$

On the other measures of mobility, no significant differences were found. This is in contrast with earlier reported findings on effects of a microprocessor-controlled knee. We believe that numerous factors have led to these discrepancies on the measures of mobility.

First of all, we included relatively active participants as is reflected in their K-level. Because the Timed "up and go" Test is known to have a ceiling effect for fit elderly people and younger people with an amputation ${ }^{185}$ it may not have been able to detect differences between prosthetic knees. This is even further highlighted when the minimal detectable change of the Timed "up and go" Test is taken into consideration, which is 3.6 seconds. ${ }^{173}$ Our participants should have increased their Timed "up and go" Test performance with over $34 \%$ to find a difference that exceeds day-to-day variation. We believe that this would have been hard, if not impossible, to achieve. Retrospectively, the L-test ${ }^{185}$ would have been a more suitable test for our study population.

Another contributing factor to the lack of differences on particularly the Hill and Stair Assessment Index might be the fact that we chose to leave out a gait training program for participants while walking with the Rheo Knee II. During the measurements some participants responded that they had no stairs or ramps in their home environment, or were re- 
luctant to descent stairs step-over-step due to safety issues. In addition, several participants preferred step-by-step because they believed this was faster. A training program might have counteracted these premises and differences in stair and ramp negotiation might have been identified. Then again, the training should have been offered to both prosthetic knee conditions, as training might have an influence on outcome. ${ }^{71}$

Our study was confounded by a number of factors, firstly of all, our sample size was low which affected statistical power, making it harder to find statistically significant differences. However, if marked differences between prosthetic knee conditions existed, these could have been identified with a small study. The presented results suggest that marked differences were not present in our population.

Secondly, we provided eight weeks of acclimatization which might have been too short to achieve full customization to the Rheo Knee II. We do not have evidence that subjects were fully acclimatised, which might have affected outcome. This is of particular importance because we compared the experience with the Rheo Knee II to 1-41 years of experience with a non-microprocessor-controlled prosthetic knee. However, evidence-based guidelines for the duration of the acclimatization period are lacking. Hubbard et al. showed that it took a 26 year old person with an amputation due to trauma three weeks to achieve a stable gait pattern after prescription of a new non-microprocessor-controlled prosthetic knee. ${ }^{156}$ To what extent these findings are transferable to older persons or acclimatisation to microprocessor-controlled knees is unclear.

Finally, we used measures of mobility that encapsulate different aspects of activities of daily life. It is however questionable how these measures correlate with functioning in daily life. A recent study found small to moderate significant negative correlations between the Timed "up and go" Test and measures of community ambulation performance (e.g. daily step count, minutes active per day). ${ }^{186}$ As far as we know, the concurrent validity of the other used outcome measures and measures of daily functioning has not been established. Accordingly, we might not have quantified the effect of the Rheo Knee II on functioning in daily life to full extent.

In conclusion, we found that the transition of a non-microprocessor-controlled prosthetic knee to the Rheo Knee II led a limited amount of significant differences on a diversity of outcome measures. Significant differences were found on the Residual Limb Health subscale and the number of steps needed to complete the Standardized Walking Obstacle Course. Due to the small difference, the latter result is not considered clinically relevant.

Future research should use outcome measures that correlate to functioning in daily life to evaluate ecologically valid differences between prosthetic knees. Ideally, these outcome measures are administered in the home environment and are as unobtrusively as possible. In addition, the sensitivity to change of outcome measures to different prosthetic knees should be clarified. Finally, a well-designed large randomized cross-over trial is needed to provide decisive evidence for the added value of microprocessor-controlled prosthetic knees. 

General discussion 


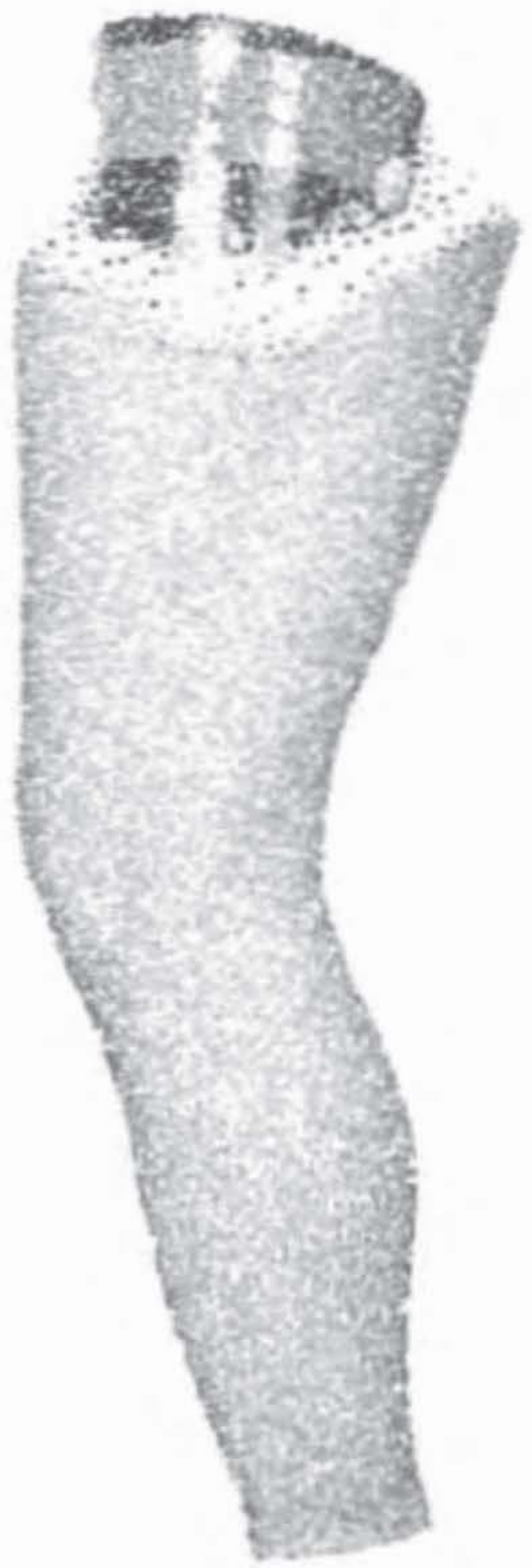



The overarching aims of this thesis were enhancing our knowledge about gait adaptations seen after amputation and study the influence of the Rheo Knee II as an example of microprocessor-controlled prosthetic knee (MPK) on gait adaptations. While each individual chapter had an aim that is related one of these overarching aims, the results of each chapter so far have been discussed individually. The general discussion combines and critically discusses the results of the chapters that are related to either one of the overarching aims. To do so, this chapter will start with a concise overview of the conclusions that can be drawn from the presented research. After this, several relevant topics relating to gait adaptations and microprocessor-controlled prosthetic knee research will be discussed. This will be followed by the strengths and limitations of this thesis. This general discussion will conclude with stating the implications for rehabilitation and the venues for future research.

\section{General CONCLUSIONS}

Individuals with a transtibial amputation and transfemoral amputation or knee disarticulation show similar adaptation strategies in the amputated and intact leg. The majority of adaptations seem to be the consequence of the loss of active ankle-foot function on the amputated side. These adaptations include increased work of the hip extensors and plantar flexors of the intact leg. Studying the muscle activation patterns of individuals with a transfemoral amputation or knee disarticulations showed additional adaptations, specifically increased activity of muscles in the residual leg during the stance to swing transition to increase socket fit.

We found that the use of the Rheo Knee II enabled participants to use the same strategies that non-amputees use to cope with platform perturbations during walking. In addition, we found that the Rheo Knee II led to increased backward margins of stability during perturbed walking when compared to the non-microprocessor-controlled prosthetic knee condition which is thought to be reflective of increased gait stability. The influence of the Rheo Knee II on the other studied aspects of gait (such as gait initiation and gait termination) was limited. At slower walking speeds, using the Rheo Knee II led to decreased vaulting of the intact ankle. Hypothetically, this could be the result of optimal prosthetic knee kinematics while using the Rheo Knee II when compared to the non-microprocessor-controlled prosthetic knee (NMPK). However, we found no statistically significant differences in peak prosthetic knee flexion during swing between the Rheo Knee II and the NMPK. In gait initiation and termination the Rheo Knee II did not lead to a reduction of intact leg reliance in respectively producing and absorbing the forces to start and stop gait. Explanations for this finding include the facts that participants did not use the knee yielding function of the Rheo Knee II and did not increase the duration of single limb support of the prosthetic leg while using the Rheo Knee II when compared to the NMPK. The Rheo Knee II had a limited effect on functional status and prosthesis-related quality of life. Using the Rheo Knee II led to a 
small, but statistically significant, increase in number of steps needed to complete an obstacle course when compared to the use of a NMPK. In addition, the Rheo Knee II positively influenced residual limb health as measured by the prosthesis evaluation questionnaire.

\section{GAIT ADAPTATIONS}

Understanding which gait adaptations occur after amputation, and more importantly, why they occur is of importance because it can provide the basis for the evaluation of (new) prosthetic components. Striving to end disability by fundamental advances in bionics, of which Hugh Herr speaks, can only be achieved when (new) prosthetic components can decrease compensational strategies of the hip of the residual leg and the ankle, knee and hip of the intact leg. While studying different outcome parameters, chapter $\mathbf{2}$ and chapter $\mathbf{3}$ both aimed to increase our understanding about gait adaptations after transfemoral amputation or knee disarticulation. In chapter 2 results of joint work and joint power were presented. These parameters provide insight into the amount of energy that is generated or absorbed around the joints of the leg. They, however, do not necessarily provide information into which muscles are responsible for the generation or absorption of energy. Relating joint work and joint power to muscle actions is not as straightforward as it might seem. Several muscles are bi-articular and can therefore generate or absorb energy over two joints. In addition, they can transfer energy over the joints they span. Based on this, it could be interesting to compare the results of chapter 2 (joint work and joint power) to the results of chapter 3 in which muscle activation patterns are presented. As chapter 3 solely focused on individuals with a transfemoral amputation or knee disarticulation, the results of the individuals with a transtibial amputation from chapter 2 are left out of this discussion.

\section{Parallels between joint power and work and muscle activation patterns}

The only parallels that can be drawn between chapter 2 and 3 are on ankle and hip level.

On ankle level, Serrousi et al. found increased work of the ankle plantar flexors of the intact leg during mid and terminal stance. ${ }^{48}$ It is thought that this is a compensation for the limited weight bearing stability and push-off most commercially available prosthetic feet can provide. In chapter 3, two striking differences in muscle activation patterns of the intact and a referent leg were visible that could relate to the finding of Seroussi et al.: (1) the soleus of the intact leg showed earlier activity when compared to the soleus of a referent leg, and (2) the anterior tibial muscle of the intact leg was active from mid-swing to the end of mid-stance, while the anterior tibial muscle of the referent leg was active from the start of the swing phase to the end of the double limb support. This led to substantial periods of co-activation in the intact leg of the agonist anterior tibial muscle and the antagonist gastrocnemius and soleus muscle. There was only limited co-contraction seen in the referent leg. Other studies have found co-contraction of the tibial anterior and gastrocnemius 
muscle in individuals without an amputation before. ${ }^{187-189}$ Di Nardo et al. studied 24 nonimpaired young individuals and found a direct relation in muscle complexity of the anterior tibial and gastrocnemius muscle: multiple bursts of the anterior tibial muscle led to multiple bursts of the gastrocnemius muscle and vice versa. ${ }^{187}$ The authors further suggest “... that the increase of complexity is required to perform further tasks as foot inversion, balance improvement during single support, control of ankle stability and knee flexion, and plays a crucial role in the improvement of joint dynamic stability." ${ }^{187}$ The findings of chapter 2 and 3 seem to point out the same mechanisms: increased activity of lower leg muscles of the intact lower leg to increase weight-bearing stability and forward progression of the intact leg.

On hip level, Seroussi et al. found increased concentric hip extensor work of the intact leg during weight acceptance when compared to a referent leg. ${ }^{48}$ Again, the study presented in chapter 3 did not focus on upper leg muscles of the intact leg. Therefore, this results of chapter 2 cannot be related to findings of chapter 3. In addition, Seroussi et al. found increased eccentric hip flexor work during mid stance of the amputated leg when compared to the intact and a referent leg. ${ }^{48}$ It is thought that this is a compensation for the impaired progression of the center of mass over the prosthetic foot. Increasing eccentric work of the hip flexors assists in preventing that the trunk and pelvis lag too far behind. In chapter 3 , muscle activation patterns of two hip flexors of the amputated leg were studied: the tensor fascia latae and the rectus femoris. Both muscles did not show activity during mid stance. This finding could be explained by the fact that these muscles are not the main hip flexors; the iliopsoas muscle is. Because of its position, the iliopsoas cannot be measured by surface electromyography and was therefore not included in the study presented in chapter 3 . The relation between the finding of Seroussi et al. could therefore not be related to findings of muscle activation patterns.

\section{Understanding why gait adaptations occur}

While chapter 2 and 3 provided insight into which gait adaptations are visible in the walking pattern of individuals with an amputation, they do not provide conclusive evidence as to why these gait adaptations occur; in chapter 2 and 3 possible explanations for the gait adaptions were based on informed judgement. Obtaining conclusive evidence as to why gait adaptations occur, requires more fundamental studies. One example of a study that provides a more in-depth understanding of the reasons gait adaptations occur was recently published. Ingraham et al. studied the effect of the presence and absence of active ankle and knee assistance on gait parameters. ${ }^{190}$ The authors found that providing active knee swing initiation decreased positive hip power of the amputated leg during early swing and negative hip power during the subsequent terminal swing phase. ${ }^{190}$ Eliminating active knee swing initiation was also found to decrease intact leg braking ground reaction forces of the intact leg. ${ }^{190}$ This finding might implicate that active knee swing initiation contributes to forward progression of the body. Providing powered plantar flexion reduced positive 
hip power of the amputated leg during early swing. As was the case with providing active knee swing initiation, the effect of this was seen in the subsequent swing phase. Providing powered plantar flexion also reduced the knee flexion and the knee extension moment in early stance of the intact leg. ${ }^{190}$ Finally, eliminating powered plantar flexion resulted in decreased braking forces of both the intact and prosthetic leg. ${ }^{190}$ The same explanation that was provided regarding active knee swing initiation seems also applicable here: by eliminating powered plantar flexion forward progression of the body is impaired requiring the intact and prosthetic leg to reduce the production of braking ground reaction forces to retain enough forward propulsion to be able to keep walking.

\section{INFLUENCE OF MPKS ON GAIT ADAPTATIONS}

The second aim of this thesis was to study the influence of a microprocessor-controlled prosthetic knee, more specifically the Rheo Knee II, on gait adaptations. We found that the use of the Rheo Knee II enabled participants to use similar strategies as non-amputees use to cope with balance perturbations. The increased backward margin of stability that we found is thought to be indicative of increased gait stability. These results are one of the first biomechanical findings that might explain the earlier reported decrease of self-reported stumbles and falls that have been associated with the use of microprocessor-controlled prosthetic knees. The Rheo Knee II had a limited influence on walking at different speeds, gait initiation and termination and quality of life, balance confidence and measures of mobility when compared to the use of a NMPK. The results of level walking, quality of life, balance confidence and measures of mobility are both in line and in contrast with the results that are described in previously conducted trials focusing on the comparison of MPKs and NMPKs. A number of factors might have played a role in the ambiguity existing in literature when it comes to the added value of a MPK on these outcome parameters. In the following subsections, factors deemed relevant are described.

\section{Differences between MPKs}

The majority of published studies focused on the comparison of the C-Leg (compact) and NMPKs. One of the reasons of the conflicting results could be that the C-Leg has a different impact on gait when compared to the use of the Rheo Knee.

As far as it is known, two publication compared the use of the Rheo Knee to the C-Leg during level walking. ${ }^{11,63}$ On metabolic energy, conflicting results are present as both a trend towards decreased metabolic energy while walking with the Rheo Knee $(P=0.092)^{11}$ as a decreased metabolic energy while walking with the C-Leg ( $p$-value unknown) ${ }^{63}$ have been described. There are no other matching outcome parameters in both publications. The reason for the conflicting findings on metabolic energy is not clear. The fact that Bellmann et al. ${ }^{63}$ solely included participants having extensive experience with the C-Leg and Johansson 
et al. ${ }^{11}$ included participants using a variety of prosthetic knees might have contributed to the conflicting results on metabolic energy. In conclusion, it is unclear whether differences in prosthetic knee design led to conflicts between the results presented in thesis and the available literature.

\section{Indication settings for the prescription of MPKs}

One of the contributing factors to the limited differences we found on group level, is that there are no known evidence-based indication settings for the prescription of microprocessor-controlled prosthetic knees on which we could base our inclusion criteria on. Because the indication criteria for the prescription of MPKs were unclear, we set broad inclusion criteria and included individuals that were willing to participate. This led to a heterogeneous population in terms of, for instance, activity level, stump length, and prosthetic loading. Activity level has been shown to influence the impact a MPK might have, ${ }^{32}$ whereas stump length has been shown to influence kinematics and muscle activation patterns of gait $^{2,10}$ and therefore, potentially, might also lead to differences in the effect of a MPK. Although not confirmed in previous studies, one can think of other factors that might also impact the influence of a MPK such as daily activity level, strength of lower limb muscles, and motor skills.

When looking at our results on the individual level, we indeed found that the Rheo Knee II had a different impact among individuals. This was especially visible in level walking at different walking speeds, quality of life (QoL), balance confidence and measures of mobility. In the results of these experiments three subgroups were visible: (1) a group in which the Rheo Knee II led to an increase of gait adaptations and reduced QoL, functional status, and balance confidence $(n=2),(2)$ a group in which no differences were found $(n=4)$, and (3) a group in which the Rheo Knee II reduced gait adaptations and increased QoL, functional status, and balance confidence $(n=4)$. Because the results are presented on group level, the positive effects that the Rheo Knee II had in certain individuals is masked by the results of individuals in which the Rheo Knee II had no or a detrimental effect. Due to the small sample size, a detailed subgroup analysis was not possible. These results seem to indicate that future research endeavors should be undertaken to clarify indication settings for the prescription of prosthetic knees by studying individual patient-prosthesis interactions. Significant progress in this field would allow the development of evidence-based guidelines for the prescription of prosthetic knees in daily clinical practice.

Since the start of the project, the Dutch workgroup amputation and prosthetics developed the protocol "recording and pricing structure of leg prostheses". ${ }^{191}$ This protocol uses the K-levels ${ }^{142}$ to grade the contemplated use of the prosthesis in case of the first prosthesis. The SIGAM-WAP ${ }^{192}$ is used to grade the contemplated use of a repeated prescription. The K-level or SIGAM-WAP score is combined with other information such as anatomical characteristics, activities and participation to select the prosthesis that best matches its contemplated use. ${ }^{191}$ This an important and paramount first step in standardizing pros- 
thetic prescription, especially as it prescribes the use of test period and clinical tests to determine the added value of MPKs. However, it is unclear what the scientific base is of the classification of the different knees in terms of K-levels is. In addition, the scientific basis for the match between the contemplated use of a prosthetic knee and the characteristics of the user seems to be lacking. This further highlights the need for research that focuses on the question which type of prosthesis is suited for which type of patient.

\section{Methodological considerations regarding prosthetic knee research}

Another explanation for the limited amount of differences that we have found might be the fact that we performed measurements in a gait lab and predominantly studied walking tasks with a low complexity.

Performing measurements in a gait lab has some limitations because it provides an artificial environment that not necessarily corresponds with the environment of in-house or community ambulation. A gait lab, for instance, usually allows ten to fifteen meters of straight-line walking, which is usually not possible in the average house. Next to that, participants are aware that they are being observed which might affect their behavior, which is known as the Hawthorne effect. A recent preliminary study investigated whether this effect was present in two individuals with a transfemoral amputation. ${ }^{193}$ The authors found that the notion that gait is observed led to a relative decrease of the first double limb support of the intact leg $(+10 \%)$, increase of gait speed $(+6 \%)$, and increase of prosthetic step length (7\%) when compared to unobserved gait. ${ }^{193}$ These results seem to indicate that the Hawthorne effect might be present in gait of individuals with a transfemoral amputation and suggest that the gait pattern measured in a gait lab does not necessarily corresponds with the gait pattern as it is present in daily life. Future research using larger study samples is needed to confirm or reject these preliminary findings.

Finally, we predominantly studied walking tasks with a relative low complexity. It could be possible that an added value of a MPK is found when walking tasks are studied with a complexity that more closely resembles the complexity of activities of daily living. This statement is further underlined by the fact that we did found differences on the most complex task that we studied: responses to anteroposterior balance perturbations. A recent study indicated that reliance on the intact leg increased during more complex tasks such as stair or ramp walking. ${ }^{194}$ An increased reliance on the intact leg might suggest that the limitations of the prosthetic leg are more pronounced during these tasks. In case non-optimal knee damping is the basis of these limitations, a MPK might be of added value during the completion of such tasks. A potential added value of a MPK during more complex tasks, as suggested by this thesis, might explain the mismatch between the limited added value of MPKs that is found in literature and the added value that has been shown on more subjective outcome measures: maybe we are measuring in a wrong way and we should perform unobtrusive instrumented analysis of activities of daily living in a meaningful context to increase the ecological validity of the measurements. 
One possible solution that would allow studying the gait pattern during more complex tasks and with decreased awareness of being observed are ambulant gait analysis or monitoring systems. In recent years, developments in ambulant gait analysis systems have taken a flight. Recently, van Meulen et al. showed that it is possible to obtain gait parameters of stroke survivors in their own home, using an ambulant gait analysis system consisting of inertial measurement units. ${ }^{195}$ They also showed that differences in spatiotemporal variables of the 10 meter walk test performed in the clinic and in participants' homes, ${ }^{195}$ suggesting that the results of a measurement performed in a clinic are not necessarily transferable to how people walk in their own home. Whether these results are also applicable for individuals with an amputation could be elucidated by future research. In addition, advanced monitoring and ambulant gait analysis systems could be used in future studies to study the impact of a MPK in a more meaningful context to increase the ecological validity of the measurements.

\section{Non-actuated prosthetic devices}

The final contributing factor that will be discussed is the fact that all the prosthetic knees that were tested have no actuation: both the Rheo Knee II and NMPKs can only dissipate energy and are not able to generate energy. The Vari-Flex ${ }^{\circledR}$ with $\mathrm{EVO}^{\mathrm{TM}}$ prosthetic foot that was used can be classified as an energy storing and releasing foot. This prosthetic foot type can store some energy during the beginning of the stance phase and release the energy during terminal stance. The amount that is stored and released, however, is substantially less than the amount of energy that is generated by the physiological ankle. In the section of this discussion describing gait adaptations it became clear that several gait adaptations are caused by the lack of active ankle and knee control of conventional prosthetic devices. Based on this, prosthetic knee devices with actuation might have a bigger impact on gait adaptations than a MPK or an energy-storing and releasing foot might have.

Hafner and Askew compared the use of NMPKs, the Rheo Knee II, and the actuated Power Knee II. ${ }^{34}$ They found that the use of a powered prosthetic knee increased the time needed to complete the timed "up-and-go" Test and time needed to ascend and descend the ramp when compared to the MPK and NMPK condition. ${ }^{34}$ In addition, the powered prosthetic knee led to a decrease in daily step activity when compared to the MPK and NMPK condition. ${ }^{34}$ Balance confidence was significantly increased while walking with the powered prosthetic knee when compared to the NMPK condition. ${ }^{34}$ Finally, they found that number of reported falls increased while using the MPK and powered prosthetic knee when compared to the NMPK condition. These negative findings combined with the high attrition of subjects in the active knee condition lead the authors to conclude that powered prosthetic knee control may not be the ideal choice for middle-age or older individuals with a transfemoral amputation. Future studies should clarify whether this initial findings holds up when powered prosthetic devices are studied in more detail. 


\section{STRENGTHS AND LIMITATIONS}

Several strength and limitation of the research that is presented in this thesis can be stated. One of the strengths of the thesis is that we tested the Rheo Knee II on a wide variety of outcome parameters and during different gait activities. Another strength is that we not only focused on biomechanics of the prosthesis but also quantified outcome parameters of the hip of the residual leg and all joints of the intact leg. Finally, we aimed to stick as close to usual care as possible by, for instance, leaving out a gait training program. While this improved the transferability of our results to daily clinical practice, it might have influenced the effect the Rheo Knee II potentially had as participants might not have been able to use the Rheo Knee II to its full potential. One of the major limitations of this thesis is the limited sample size. This affected statistical power and thereby the possibility to find statistical significant differences. In addition, because indication setting for microprocessorcontrolled prosthetic knees are unclear it would have been interesting to see whether the effect of the Rheo Knee II was different in subgroups. However, our study sample did not allow a sub-group analysis. An additional limitation is that we were not able to quantify kinetic variables during the level walking and platform perturbations experiments due to the fact that we used a single-belt instrumented treadmill. From the gait adaptations part it became clear that a substantial amount of gait adaptations are visible in kinetic outcome parameters. It is therefore unfortunate that we were not able to quantify the influence of the Rheo Knee II on joint moments, power, and/or work. A final limitation is that all measurements were performed in an artificial and the majority of the studied tasks had a low complexity. This limits the ecological validity of our measurements.

\section{IMPLICATIONS FOR REHABILITATION}

The results of this further strengthened the notion that individuals with an amputation heavily rely on their intact leg. This led to clear asymmetry in kinematic and kinetic variables of the intact and prosthetic leg. In the discussion of this thesis, results of trials have been described that suggest that these asymmetries are functional in part. These results indicate that rehabilitation that is aimed to increase gait symmetry based on the assumption that gait symmetry is always more functional should be discouraged. While this was already noted by Winter and Sienko in $1988,{ }^{49}$ gait symmetry still often comes up as goal of rehabilitation when talking to physical therapists and prosthetists. An often heard question in these discussion is what the goal of rehabilitation should be in case gait symmetry is discarded. While this thesis does not provide a clear answer to this question, we believe that rehabilitation should focus on training the muscle groups that show increased activity to allow maximal adaptability of these muscles. In the intact leg these include the ankle plantar flexors, knee extensors, and the hip flexors and extensors. The same applies for the hip 
flexors and extensors of the amputated leg. In addition, we found that the use of the Rheo Knee II enabled the use of strategies to cope with balance perturbations that are also used by non-amputees. These include a decreased step length and foot forward placement. The rehabilitation of individuals walking with the Rheo Knee II, thus, can include exercises in which these strategies are actively learned to maximize gait stability and minimize fall risk.

\section{IMPLICATIONS FOR FUTURE RESEARCH}

In this general discussion, a number of implications for future research have been proposed. In summary, we propose that future research could focus on (1) increasing our understanding of movement control strategies of individuals with a lower limb amputation, (2) quantify the influence of actuated prosthetic components on the gait pattern of individuals with a transfemoral amputation or knee disarticulation, (3) increase the ecological validity of measurements by measuring more complex and meaningful gait activities in the context in which they are usually carried out, (4) focus on the research question "which type of prosthesis for which type of patient" to allow tailoring of individual prosthetic prescription. In addition, ambulatory assessment of the gait pattern of an individual with a transfemoral amputation over a prolonged period of time can lead to increased insight into how individuals adapt to a prosthetic device. This could also provide insight into how individuals with an amputation interact with intelligent devices. Combining this information with the information that can be obtained from the prosthetic device itself (such as torques and angles) could provide insight into the the complex patient-prosthesis interactions on individual level. This information can be used to further personalize prosthetic prescription and allow patient-driven development of prosthetic devices.

\section{AdAPTING TO CHANGE?}

Individuals have to adapt on several levels after their amputation. First of all, they have to adapt to the amputation itself. Next, they have to adapt to a prosthetic device that is usually not capable of providing a full substitution of the amputated body part. Finally, when walking with a prosthetic device with artificial intelligence, individuals have to adapt to a prosthesis that adapts to the user. One has to have knowledge of all levels to fully grasp in what way a microprocessor-controlled prosthetic knee influences the gait pattern of individuals with a transfemoral amputation or knee disarticulation. The difficulty to adequately substitute parts in a biological system of substantial complexity reveals the discrepancies between what we think we know and the mechanisms that we overlook or are still poorly understood. Therefore this field of study might also need to adapt. Adapt to first understand why the gait pattern is the way it is before investigating how new prosthetic devices can influence aspects of gait we do not fully comprehend yet. 
CHAPTER 9: General Discussion

"Indeed, through fundamental advances in bionics in this century, we will set the technological foundation for an enhanced human experience and we will end disability"

Hugh Herr 

References

Summary

Samenvatting

Dankwoord

About the author

Progress range 


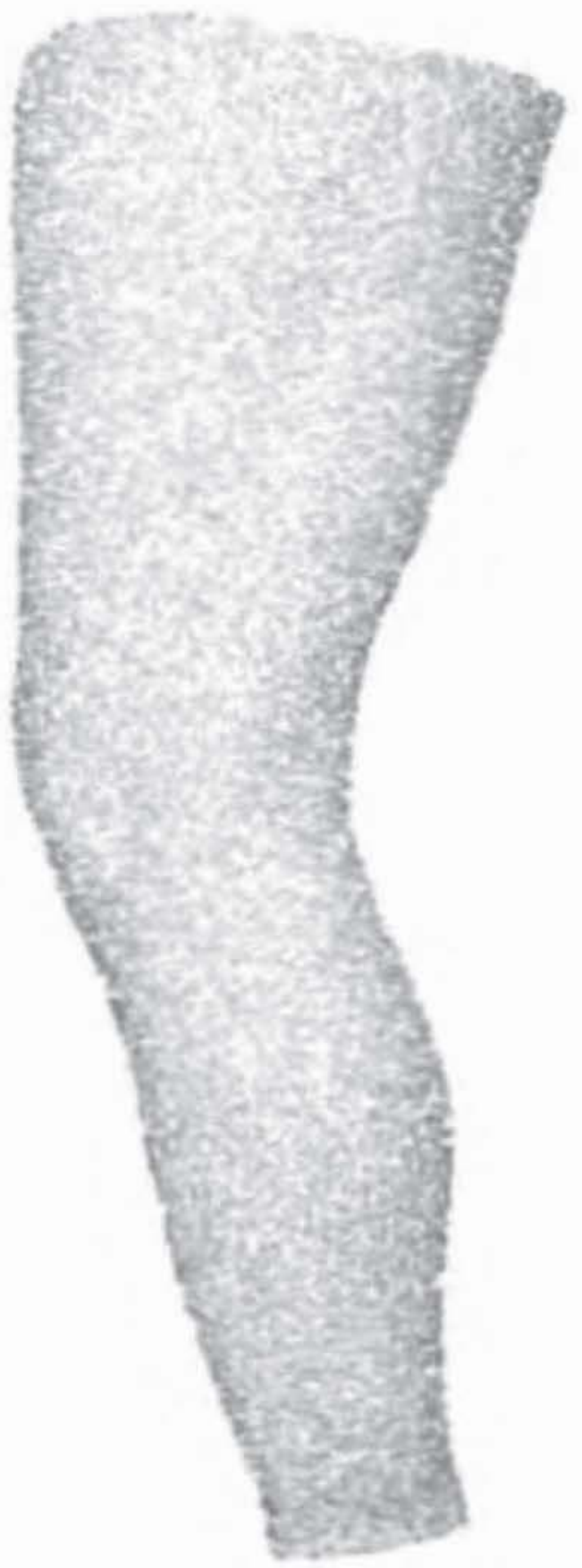


1. Herr H. The new bionics that let us run, climb and dance. www.ted.com/talks/hugh_herr the_new_bionics_that_let_us_run_climb_and_dance. Accessed: 26-03-2016; 2014.

2. Jaegers SM, Arendzen JH, de Jongh HJ. An electromyographic study of the hip muscles of transfemoral amputees in walking. Clin Orthop Relat Res. 1996;328:119-28.

3. Perry J. Gait analysis: normal and pathological function. SLACK Incorporated; 1992.

4. ISO. Prosthetics and orthotics - Classification and description of prosthetic components - Part 2: Description of lower limb prosthetic components. ISO; 13405-2:2015(E).

5. ISO. Prosthetics and orthotics - Classification and description of prosthetic components - Part 1: Classification of prosthetic components. ISO; 13405-1:2015(E).

6. Gard SA, Childress DS. The influence of stance-phase knee flexion on the vertical displacement of the trunk during normal walking. Arch Phys Med Rehabil. 1999;80:26-32.

7. Herr $\mathrm{H}$, Wilkenfield A. User-adaptive control of a magnetorheological prosthetic knee. Ind Robot. 2003;30:42-55.

8. Peeraer L, Tilly K, der Perr GV. A computer-controlled knee prosthesis: a preliminary report. J Med Eng Technol. 1989;13:134-5.

9. Murray MP, Sepic SB. Gait patterns of above-knee amputees using constant-friction knee components. Bull Prosthet. 1980;17:35-45.

10. Jaegers $\mathrm{SMHJ}$, Arendzen JH, de Jongh $\mathrm{HJ}$. Prosthetic gait of unilateral transfemoral amputees: a kinematic study. Arch Phys Med Rehabil. 1995;76:736-43.

11. Johansson JL, Sherill DM, Riley PO, Bonato P, Herr H. A clinical comparison of variable-damping and mechanically passive prosthetic knee devices. Am J Phys Med Rehabil. 2005;84:563-75.

12. Segal AD, Orendurff MS, Klute GK, McDowell ML, Pecoraro JA, Shofer J, et al. Kinematic and kinetic comparisons of transfemoral amputee gait using C-Leg and Mauch SNS prosthetic knees. J Rehabil Res Dev. 2006;43:857-69.

13. Winter DA. Energy generation and absorption at the anke and knee during fast, natural and slow cadences. Clin Orthop Rel Res. 1983;175:147-54.

14. Miller WC, Speechley M, Deathe B. The prevalence of risk factors of falling and fear of falling among lower extremity amputees. Arch Phys Med Rehabil. 2001;82:1031-1037.

15. Murray MP, Mollinger LA, Sepic SB, Gardner GM, Linder MT. Gait patterns in above-knee amputee patients: hydraulic swing control vs constant-friction knee components. Arch Phys Med Rehabil. 1983;64:339-45.

16. Nolan $\mathrm{L}$, Lee $A$. The functional demands on the intact limb during walking for active trans-femoral and trans-tibial amputees. Prosthet Orthot Int. 2000;24:117-25.

17. Gitter A, Czerniecki J, Weaver K. A reassessment of center-of-mass dynamics as a determinate of the metabolic inefficiency of above-knee amputee ambulation. Am J Phys Med Rehabil. 1995;74:332-8.

18. Czerniecki JM. Rehabilitation in limb deficiency. 1. Gait and motion analysis.. Arch Phys Med Rehabil. 1996;77:S3-8.

19. Smith D, Michaels J, Bowker J. Atlas of amputation and limb deficiencies. American Academy of Orthopaedic Surgeons; 2002.

20. Stinus $\mathrm{H}$. Biomechanik und beurteilung des mikroprozessorgesteurten exoprothesengelenkes C-Leg. Z Orthop. 2000;138:278-82.

21. Össur. Rheo Knee. Össur Prosthetics Product Catalog. Össur Americas; 2011.

22. Sawers AB, Hafner BJ. Outcomes associated with the use of microprocessor-controlled pros- 
thetic knees among individuals with unilateral limb loss: a systematic review. J Rehabil Res Dev. 2013;50:273-314.

23. Highsmith MJ, Halhe JT, Bongiorni DR, Sutton BS, Groer S, Kaufman KR. Safety, energy efficiency, and cost efficacy of the C-Leg for transfemoral amputees: a review of the literature. Prosthet Orthot Int. 2010;34:362-77.

24. Kannenberg A, Zacharias B, Pröbsting E. Benefits of microprocessor-controlled prosthetic knees to limited community ambulators: systematic review. J Rehabil Res Dev. 2014;51:1469-96.

25. Orendurff MS, Segal AD, Klute GK, McDowell ML, Pecoraro JA, Czerniecki JM. Gait efficiency using the C-Leg. J Rehabil Res Dev. 2006;43:239-46.

26. Kahle JT, Highsmith MJ, Hubbard SL. Comparison of nonmicroprocessor knee mechanism versus C-Leg on Prosthesis Evaluation Questionnaire, stumbles, falls, walking tests, stair descent, and knee preference. J Rehabil Res Dev. 2008;45:1-14.

27. Hafner BJ, Willingham LL, Buell NC, Allyn KJ, Smith DG. Evaluation of function, performance and preference as transfemoral amputees transition from mechanical to microprocessor control of the prosthetic knee. Arch Phys Med Rehabil. 2007;88:207-17.

28. Jepson F, Datta D, Harris I, Heller B, Howitt J, McLean J. A comparative evaluation of the Adaptive knee and Catech knee joints: a preliminary study. Prosthet Orthot Int. 2008;32:84-92.

29. Kaufman KR, Levine JA, Brey RH, Iverson BK, McCrady SK, Padgett DJ, et al. Gait and balance of transfemoral amputees using passive mechanical and microprocessor-controlled prosthetic knees. Gait Posture. 2007;26:489-93.

30. Mâaref K, Martinet N, Grumillier C, Ghannouchi S, André JM, Paysant J. Kinematics in the terminal swing phase of unilateral transfemoral amputees: microprocessor-controlled versus swingphase control prosthetic knees. Arch Phys Med Rehabil. 2010;91:919-25.

31. Schaarschmidt M, Lipfert SW, Meier-Gratz C, Scholle HC, Seyfarth A. Functional gait asymmetry of unilateral transfemoral amputees. Hum Mov Sci. 2012;31:907-17.

32. Hafner BJ, Smith DG. Differences in function and safety between medical functional classification level-2 and -3 transfemoral amputees and influence of prosthetic knee joint control. J Rehab Res Dev. 2009;46:417-34.

33. Seymour R, Engbretson B, Kott K, Ordway N, Brooks G, Crannell J, et al. Comparison between the C-leg microprocessor-controlled prosthetic knee and non-microprocessor control prosthetic knees: a preliminary study of energy expenditure, obstacle course performance, and quality of life survey. Prosthet Orthot Int. 2007;31:51-61.

34. Hafner BJ, Askew RL. Physical performance and self-report outcomes associated with use of passive, adaptive, and active prosthetic knees in persons with unilateral, transfemoral amputation: randomized crossover trial. J Rehabil Res Dev. 2015;52:677-700.

35. Meier MR, Hansen AH, Gard SA, McFadyen AK. Users' maneuverability and movement efficiency when using Otto Bock C-Leg, Otto Bock 3R60, and CaTech SNS prosthetic knee joints. J Rehab Res Dev. 2012;49:583-96.

36. Highsmith MJ, Kahle JT, Miro RM, Mengelkoch LJ. Ramp descent performance with the C-Leg and interrater reliability of the Hill Assessment Index. Prosthet Orthot Int. 2013;37:362-8.

37. Bell EM, Pruziner AL, Wilken JM, Wolf EJ. Performance of conventional and X2 prosthetic knees during slope descent. Clin Biomech. 2016;33:26-31.

38. Berry D, Olson M, Larntz K. Perceived stability, function and satisfaction among transfemoral amputees using microprocessor and nonmicroprocessor controlled prosthetic knees: a multicenter 
study. Prosthet Orthot Int. 2009;21:32-42.

39. Stevens PM, Carson R. Case report: using the Activities-specific Balance Confidence scale to quantify the impact of prosthetic knee choice on balance confidence. J Prosthet Orthot. 2007;19:114-116.

40. Seelen H, Hemmen B, Schmees A, Ament A, Evers S. Cost and consequences of a prosthesis with an electronically stance and swing phase controlled knee joint. Technol Disabil. 2009;21:25-34.

41. Kaufman KR, Levine JA, Brey RH, McCrady SL, Padgett DJ, Joyner MJ. Energy expenditure and activity of transfemoral amputees using mechanical and microprocesso-controlled prosthetic knees. Arch Phys Med Rehabil. 2008;89:1380-5.

42. Gerzeli S, Torbia A, Fattore G. Cost utility analysis of knee prosthesis with complete microprocessor control (C-Leg) compared with mechanical technology in trans-femoral amputees. Eur J Health Econ. 2009;10:47-55.

43. Brodtkorb TH, Henrikson M, Johannesen-Munk K, Thidell F. Cost-effectiveness of C-Leg compared with non-microprocessor-controlled knees: a modelling approach. Arch Phys Med Rehabil. 2008;89:24-30.

44. Bellmann M, Schmalz T, Ludwigs E, Blumentritt S. Immediate effects of a new microprocessor controlled prosthetic knee joint: a comparative biomechanical evaluation. Arch Phys Med Rehabil. 2012;93:541-9.

45. Uchityl J, Jandacka D, Zahradnik D, Farana R, Janura M. Temporal-spatial parameters of gait in transfemoral amputees: Comparison of bionic and mechanically passive knee joints. Prosthet Orthot Int. 2013;38:199-203.

46. Rossignol S, Dubuc R, Gossard JP. Dynamic sensorimotor interactions in locomotion. Physiol Rev. 2006;86:89-154.

47. Zajac FE, Neptune RR, Kautz SA. Biomechanics and muscle coordination of walking part I: introduction to concepts, power transfer, dynamics and simulations. Gait Posture. 2002;16:215-32.

48. Seroussi RE, Gitter A, Czerniecki JM, Weaver K. Mechanical work adaptations of above-knee amputee ambulation. Arch Phys Med Rehabil. 1996;77:1209-14.

49. Winter DA, Sienko SE. Biomechanics of below-knee amputee gait. J Biomech. 1988;21:361-7.

50. Anderson FC, Pandy MG. Individual muscle contributions to support in normal walking. Gait Posture. 2003;17:159-69.

51. Gotschall JS, Kram K. Energy cost and muscular activity required for propulsion during walking. J Appl Physiol. 2003;94:1766-72.

52. Liu MQ, Anderson FC, Pandy MG, Delp SL. Muscle that support the body also modulate forward progression during walking. J Biomech. 2006;34:2623-30.

53. Neptune RR, Kautz SA, Zajac FE. Contributions of the individuals plantar flexors to support, forward progression and swing inititation during walking. J Biomech. 2001;34:1387-98.

54. Hof AL, Nauta J, van der Knaap ER, Schallig MA, Struwe DP. Calf muscle work and segment energy changes in human treadmill walking. J Electromyog Kinesiol. 1993;2:203-16.

55. Anderson FC, Goldberg SR, Pandy MG, Delp SL. Contributions of muscle forces and toe-off kinematics to peak knee flexion during swing phase of normal gait: an induced position analysis. J Biomech. 2004;37:731-7.

56. Gitter A, Czerniecki JM, DeGroot DM. Biomechanical analysis of the influence of prosthetic feet on below-knee amputee walking. Am J Phys Med Rehabil. 1991;70:142-8.

57. Silverman AK, Fey NP, Portillo A, Walden JG, Bosker G. Compensatory mechanicsms in below- 
knee amputee gait in response to increasing steady-state walking speeds. Gait Posture. 2008;28:602-9.

58. Sadeghi H, Allard P, Duhaime M. Muscle power compensatory mechanicsms in below-knee amputee gait. Am J Phys Med Rehabil. 2001;80:25-32.

59. Bae TS, Choi K, Hong D, Mun M. Dynamic analysis of above-knee amputee gait. Clin Biomech. 2007;22:557-66.

60. Downs SH, Black N. The feasibility of a checklist for the assessment of the methodological quality both of randomized and non-randomized studies of health care interventions. J Epidiomiol Community Health. 1998;52:377-84.

61. Higgins JP, Green S, editors. Cochrane handbook for systematic reviews of interventions version 5.0.2. Available from http://handbook.cochrane.org: The Cochrane Collaboration; 2009.

62. Eilenberg MF, Geyer H, Herr H. Control of a powered ankle-foot prosthesis based on a neuromuscular model. IEEE Trans Neural Syst Rehabil Eng. 2010;18:164-73.

63. Bellmann M, Schmalz T, Blumentritt S. Comparative biomechanical analysis of current microprocessor-controlled prosthetic knee joints. Physiol Rev. 2010;91:644-52.

64. Grabowski AM, Rifkin J, Kram R. K3 Promotor prosthetic foot reduces the metabolic cost of walking for unilateral transtibial amputees. J Prosthet Orthot. 2010;22:113-120.

65. Vanicek N, Strike S, McNaughton L, Polmam R. Gait patterns in transtibial amputee fallers vs non-fallers: biomechanical differences during level walking. Gait Posture. 2009;29:415-20.

66. Beyaert C, Grumillier C, Martinet N, Paysant J, André JM. Compensatory mechanism involving the knee joint of the intact limb during gait in unilateral below-knee amputees. Gait Posture. 2008;28:278-84.

67. Andrysek J, Redekop S, Naumann S. Preliminary evaluation of an automatically stance-phase controlled pediatric prosthetic knee joint using quantitative gait analysis. Arch Phys Med Rehabil. 2007;88:464-70.

68. Goujon H, Bonnet X, Sautreuil P, Maurisset M, Darmon L, Fode P, et al. A functional evaluation of prosthetic foot kinematics during lower-limb amputee gait. Prosthet Orthot Int. 2006;30:213-23.

69. Martinez-Villalpando EC, Herr H. Agonist-antagonist active knee prosthetsis: a preliminary study in level-ground walking. J Rehabil Res Dev. 2009;46:361-73.

70. Detrembleur C, Vanmarsenille JM, Cuyper FD, Dierick F. Relationship between energy cost, gait speed, vertical displacement of centre of body mass and efficiency of pendulum-like mechanism in unilateral amputee gait. Gait Posture. 2005;21:333-40.

71. Sjödahl C, Jarnlo GB, Söderberg B, Persson BM. Kinematic and kinetic gait analysis in the sagittal plane of transfemoral amputees before and after special gait re-eduction. Prosthet Orthot Int. 2002;26:101-12.

72. Selles RW, Korteland S, Soest AJV, Bussmann JB, Stam HJ. Lower-leg inertial properties in transtibial amputees and control subjects and their influence on the swing phase during gait. Arch Phys Med Rehabil. 2003;84:569-77.

73. Sjödahl C, Jarnlo GB, Soderberg B, Persson BM. Pelvic motion in trans-femoral amputees in the frontal and transverse plane before and after special gait re-education. Prosthet Orthot Int. 2002;26:101-12.

74. Blumentritt S, Schmalz T, Jarasch R. Die Bedeuting des statischen Prosthesenaufbaus fãijr das Stehen und Gehen des Unterschenkelamputierten. OrthopÃd'de. 2001;30:161-8.

75. Hillery SC, Wallace ES. Trans-tibial amputee gait adaptations as a result of prosthetic inertial 
manipulation. Disabil Rehabil. 2000;22:383-6.

76. Quesada PM, Pitkin M, Colvin J. Biomechanical evaluation of a prototype foot/ankle prosthesis. IEEE Trans Rehabil Eng. 2000;8:156-9.

77. Prince F, Winter DA, Sjonnensen G, Powell C, Wheeldon RK. Mechanical efficiency during gait of adults with transtibial amputation: a pilot study comparing the $\mathrm{SACH}$, Seattle, and Golden-Ankle prosthetic feet. J Rehabil Res Dev. 1998;35:177-85.

78. Cortes A, Viosca E, Hoyos JV, Prat J, Sanchez-Lacuesta J. Optimisation of the prescription for trans-tibial (TT) amputees. Prosthet Orthot Int. 1997;21:168-74.

79. Schneider K, Hart T, Zernicke RF, Setoguchi Y, Oppenheim W. Dynamics of below-knee child amputee gait: SACH foot versus flex foot. J Biomech. 1993;26:1191-204.

80. Lemaire ED, Fisher FR, Robertson DG. Gait patterns of elderly men with trans-tibial amputations. Prosthet Orthot Int. 1993;17:27-37.

81. Colborne GR, Naumann S, Longmuir PE, Berbrayer D. Analysis of mechanical and metabolic factors in the gait of congenital below knee amputees: a comparison of the SACH and Seattle feet. Am J Phys Med Rehabil. 1992;71:272-8.

82. Hale SA. Analysis of the swing phase dynamics and muscular effort of the above-knee amputee for varying prosthetic shank loads. Prosthet Orthot Int. 1990;14:125-35.

83. Hurley GR, McKenney R, Robinson M, Zadravec M, Pierrynowski MR. The role of the contralateral limb in below-knee amputee gait. Prosthet Orthot Int. 1990;14:33-42.

84. Lewallen R, Dyck G, Quanbury A, Ross K, Letts M. Gait kinematics in below-knee child amputees: a force plate analysis. J Pediatr Orthop. 1986;6:291-8.

85. Goh JC, Solomonidis SE, Spence WD, Paul JP. Biomechanical evaluation of SACH and uniaxial feet. Prosthet Orthot Int. 1984;8:147-54.

86. White H, VandenBrink K, Augsburger S, Cupp T, Cottle W, Tylkowski C. Bilateral kinematic and kinetic adata of two prosthetic designs: a case study. J Prosthet Orthot. 2000;12:120-6.

87. Oberg K, Lanshammer $\mathrm{H}$. An investigation of kinematic and kinetic variables for the description of prosthetic gait using the ENOCH system. Prosthet Orthot Int. 1982;6:43-7.

88. Barnett C, Vanicek N, Polman R, Hancock A, Brown B, Smith L, et al. Kinematic giat adaptations in unilateral transtibial amputees furing rehabilitation. Prosthet Orthot Int. 2009;33:135-47.

89. Sutherland JL, Sutherland DH, Kaufman KR, Teel M. Case study forum: gait comparison of two prosthetic knee units. J Prosthet Orthot. 1997;9:168-73.

90. Baum BS, Schnall BL, Tis JE, Lipton JS. Correlation of residual limb length and gait parameters in amputees. Injury. 2008;39:728-33.

91. Sapin E, Goujon H, de Almeida F, Fode P, Lavaste F. Functional gait analysis of trans-femoral amputees using two different single-axis prosthetic knees with hydraulic swing-phase control: kinematic and kinetic comparison of two prosthetic knees. Prosthot Orthot Int. 2008;32:201-18.

92. Thomas SS, Buckon CE, Helper D, Turner N, Moor M, Krajbich JI. Comparison of the Seattle lite foot and Genesis II prosthetic foot during walking and running. J Prosthet Orthot. 2000;12:9-14.

93. McMulkin ML, Osebold WR, Mildes RD, Rosenquist RD. Comparison of three prediartric prosthetic feet during functional activities. J Prosthet Orthot. 2004;16:78-86.

94. Chow $\mathrm{DH}$, Holmes AD, Lee CKL, Sin SW. The effect of prosthesis alignment on the symmetry of gait in subjects with unilateral transtibial amputation. Prosthet Orthot Int. 2006;30:114-28.

95. Burkett B, Smeathers J, Barker T. Walking and running inter-limb asymmetry for paralympic trans-femoral amputees, a biomechanical analysis. Prosthet Orthot Int. 2003;2003:36-47. 
96. Silver-Thorn B, Glaister CL. Functional stability of transfemoral amputee gait using the $3 R 80$ and Total Knee 2000 prosthetic knee units. J Prosthet Orthot. 2009;21:18-31.

97. Powers CM, Rao S, Perry J. Knee kinetics in trans-tibial amputee gait. Gait Posture. 1998;8:1-7.

98. Tesio L, Lanzi D, Detrembleur C. The 3-D motion of the centre of gravity of the human body during level walking. II. Lower limb amputees. Clin Biomech. 1998;13:83-90.

99. Sanderson DJ, Martin PE. Lower extremity kinematic and kinetic adaptations in unilateral belowknee amputees during walking. Gait Posture. 1997;6:126-36.

100. Postema K, Hermens HJ, Vries JD, Zilvold G, Grootenboer HJ, Koopman HFJM, et al. Energy storing properties of four types of prosthetic feet. J Rehabil Sci. 1994;7:49-54.

101. Hosoda M, Yoshimure O, Takayanagi K, Maejima H, Kobayashi R, Minematsu A, et al. Human gait analysis as viewed from $A / K$ and $B / K$ force plate/stick figures. J Phys Ther Sci. 1999;11:79-85.

102. Ishai $G, B a r A$. Evaluation of AK prostheses comparing conventional with adaptive knee control devices. J Biomed Eng. 1984;6:27-32.

103. Bateni H, Olney SJ. Kinematic and kinetic variations of below-knee amputee gait. J Prosthet Orthot. 2002;14:2-12.

104. Lee WC, Forssard LA, Hagberg K, Brånemark R, Evans JH, Pearcy MJ. Kinetics of transfemoral amputees with osseointegrated fixation performing common activities of daily living. Clin Biomech. 2007;22:665-73.

105. Scherer RF, Downling JJ, Frost G, Robinson M, McLean K. Mechanical and metabolic work of persons with lower-extremity amputations walking with titanium and stainless steel prostheses: a preliminary study. J Prosthet Orthot. 1999;11:38-42.

106. Centomo H, Amarantini D, Martin L, Prince F. Muscle adaptation patterns of children with a trans-tibial amputation during walking. Clin Biomech. 2007;22:457-63.

107. Blumentritt S, Schmalz T, Jarasch R. The safety of C-leg; biomechanical tests. J Prosthet Orthot. 2009;21:2-17.

108. Blumentritt S, Scherer HW, Michael JW, Schmalz T. Transfemoral amputees wlaking on a rotary hydraulic prosthetic knee mechanism: a preliminary report. J Prosthet Orthot. 1998;10:61-70.

109. Bae TS, Choi K, Mun M. Level walking and stair climbing gait in above-knee amputees. J Med Eng Technol. 2009;33:130-5.

110. Grumillier C, Martinet N, Paysant J, André JM, Beyaert C. Compensatory mechanism involving the hip joint of the intact limb during gait in unilateral trans-tibial amputees. J Biomech. 2008;41:2926-31.

111. Lloyd CH, Stanhope SJ, Davis IS, Royer TD. Strength asymmetry and osteoarthritis risk factors in unilateral trans-tibial amputee gait. Gait Posture. 2010;32:296-300.

112. Norvell D, Czerniecki J, Reiber G, Maynard C, Pecoraro J, Weiss N. The prevalence of knee pain and symptomatic knee osteoarthritis among veteran traumatic amputees and nonamputees. Arch Phys Med Rehabil. 2005;86:487-93.

113. Zmitrewicz RJ, Neptune RR, Sasaki K. Mechanical energetic contributions from individual muscles and elastic prosthetic feet during symmetric unilateral transtibial amputee walking: a theoretical study. J Biomech. 2007;40:1824-31.

114. Barr AE, Siegel KL, Danoff JV, 3rd CLM, Tomasko A, Sable I, et al. Biomechanical comparison of the energy-storing capabilities of SACH and Carbon Copy II prosthetic feet during the stance phase of gait in a person with below-knee amputation. Phys Ther. 1992;72:344-54.

115. Torburn L, Perry J, Ayyappa E, Shanfield SL. Below-knee amputee gait with dynamic elastic re- 
sponse prosthetic feet: a pilot study. J Rehabil Res Dev. 1990;27:369-84.

116. Underwood HA, Tokuno CD, Eng JJ. A comparison of two prosthetic feet on multi-joint and multiplane kinetic gait compensations in individuals with a unilateral trans-tibial amputation. Clin Biomech. 2004;19:609-16.

117. Lelas JL, Merriman GJ, Rileyand PO, Kerrigan DC. Predicting peak kinematic and kinetic parameters from gait speed. Gait Posture. 2003;17:106-12.

118. Grimes DA, Schulz KF. Bias and causal associations in observational research. Lancet. 2002;359:248-52.

119. Vrieling AH, van Keeken HG, Schoppen T, Hof AL, Otten B, Halbertsma JPK, et al. Gait adjustments in obstacle crossing, gait initiation and gait termination after a recent lower limb amputation. Clin Rehabil. 2009;23:659-71.

120. Prinsen EC, Nederhand MJ, Rietman JS. Adaptation strategies of the lower extremities of patients with a transtibial or transfemoral amputation during level walking: a systematic review. Arch Phys Med Rehabil. 2011;92:1311-25.

121. Huang S, Ferris DP. Muscle activation patterns during walking from transtibial amputees recorded within the residual limb-prosthetic interface. J Neuroeng Rehabil. 2012;9:1-16.

122. Hong JH, Mun MS. Relationship between socket pressure and EMG of two muscles in transfemoral stumps during gait. Prosthet Orthot Int. 2005;29:59-72.

123. Hermens HJ, Freriks B, Merletti R, Stegeman D, Blok J, Rau G, et al. European recommendations for surface electromyography. Roessingh Research and Development; 1999.

124. Wentink EC, Beijen SI, Hermens HJ, Rietman JS, Veltink PH. Intention detection of gait initiation using EMG and kinematic data. Gait Posture. 2012;37:223-8.

125. Watanabe T, Saito H, Koike E, Nitta K. A preliminary test of measurement of joint angles and stride length with wireless inertial sensors for wearable gait evaluation system. Comput Intell Neurosci. 2011;2011:975193.

126. Chan ADC, Green GC. Myoelectric control development toolbox. In: Proceedings 30th Conference of the Canadian Medical \& Biological Engineering Society. Toronto; 2007. p. M0100.

127. Zardoshti-Kermani M, Wheeler BC, Badie K, Hashemi RM. EMG feature evaluation for movement control of upper extremity protheses. IEEE Trans Rehabil Eng. 1995;3:324-33.

128. Mickelborough J, van der Linden ML, Tallis RC, Ennos AR. Muscle activity during gait initiation in normal elderly people. Gait Posture. 2004;19:50-7.

129. Hershler C, Morris M. An optimally criterion for processing electromyography (EMG) signals relating to human locomotion. IEEE Trans Biomed Eng. 1978;25:413-20.

130. Malone A, Meldrum D, Gleeson J, Bolger C. Reliability of surface electromyography timing parameters in gait in cervical spondylotic myelopathy. J Electromygr Kinesiol. 2011;21:1004-10.

131. Nene A, Byrne $C$, Hermens $H$. Is rectus femoris really a part of quadriceps? Assessment of rectus femoris function during gait in able-bodied adults. Gait Posture. 2004;20:1-13.

132. Byrne CA, Lyons GM, Donnelly AE, O'Keeffe DT, Hermens HJ, Nene A. Rectus femoris surface myoelectric signal cross-talk during static contractions. J Electromyogr Kinesiol. 2005;15:564-75.

133. Rose J, Gamble JG. Human Walking. 3rd ed. Lippincott Williams and Wilkins; 2006.

134. Granata KP, Padua DA, Abel M. Repeatability of surface EMG during gait in children. Gait Posture. 2005;22:346-50.

135. Burden AM, Trew M, Balzopoulos V. Normalisation of gait EMGs: a re-examination. J Electromyogr Kinesiol. 2003;13:519-32. 
136. Merletti R, Parker P. Electromyography: Physiology, engineering and noninvasive applications. Wiley Interscience; 2004.

137. Monaco V, Ghionzoli A, Micera S. Age-related modifications of muscle synergies and spinal cord activity during locomotion. J Neurophysiol. 2010;104:2092-2102.

138. Buurke JH, Hermens HJ, Erren-Wolters CV, Nene AV. The effect of walking aids on muscle activation patterns during walking in stroke patients. Gait Posture. 2005;22:164-70.

139. Datta D, Heller B, Howitt J. A comparative evaluation of oxygen consumption and gait pattern in amputees using Intelligent Prosthesis and conventionally damped knee swing-phase control. Clin Rehabil. 2005;19:398-403.

140. Arnold AS, Thelen DG, Schwartz MH, Andersen FC, Delp SL. Muscular coordination of knee motion during the terminal-swing phase of normal gait. J Biomech. 2007;40:3314-24.

141. Arnold AS, Schwartz MH, Thelen DG, Delp SL. Contributions of muscles to terminal-swing knee motions vary with walking speed. J Biomech. 2007;40:3660-71.

142. US Department of Commerce NTIS. Centers for Medicare and Medicaid Services. US Department of Health and Human Services. HFCA Common Procedure Coding Sytem (HCPCS). Springfield (VA); 2001.

143. Prinsen EC, Nederhand MJ, Koopman HFJM, Rietman JS. Added value of a user-adaptive prosthetic knee in gait initiation: off to a good start? IEEE Int Conf Rehabil Robot. 2015;p. 31-6.

144. Eberly VJ, Mulroy SJ, Gronley JK, Perry J, Yule WJ, Burnfield JM. Impact of a stance phase microprocessor-controlled knee prosthesis on level walking in lower functioning individuals with a transfemoral amputation. Prosthet Orthot Int. 2014;38:477-55.

145. Klute GK, Berge JS, Orendurff MS, Williams RM, Czerniecki JM. Prosthetic intervention effects on activity of lower-extremity amputees. Arch Phys Med Rehabil. 2006;87:717-22.

146. Michel V, Do MC. Are stance plantar flexor muscles necessary to generate propulsive forces during gait initiation? Neurosci Lett. 2002;325:139-43.

147. Michel V, Chong RK. The strategies to regulate and modulate the propulsive forces during gait initiation in lower limb amputees. Exp Brain Res. 2004;158:356-65.

148. Vrieling AH, van Keeken HG, Schoppen T, Otten B, Halbertsma JP, Hof AL, et al. Gait initiation in lower limb amputees. Gait Posture. 2008;27:423-30.

149. van Keeken HG, Vrieling AH, Hof AL, Halbertsma JP, Schoppen T, Postema K, et al. Controlling propulsive forces in gait initiation in transfemoral amputees. J Biomech Eng. 2008;130:011002.

150. van Keeken HG, Vrieling AH, Hof AL, Postema K, Otten B. Stabilizing moments of force on a prosthetic knee during stance in the first steps after gait initiation. Med Eng Phys. 2012;34:7339.

151. Nissan M. The initiation of gait in lower limb amputees: some related data. J Rehabil Res Dev. 1991;28:1-12.

152. Hansen AH, Miff SC, Childress DS, Gard SA, Meier MR. Net external energy of the biologic and prosthetic ankle during gait initiation. Gait Posture. 2010;31:13-17.

153. Brenière Y, Do MC. Control of gait initiation. J Mot Behav. 1991;23:235-40.

154. Pai YC, Patton J. Center of mass velocity-position predictions for balance control. J Biomech. 1997;30:347-54.

155. Hof AL, van Bockel RM, Schoppen T, Postema K. Control of lateral balance in walking: Experimental findings in normal subjects and above-knee amputees. Gait Posture. 2007;25:250-8.

156. English RD, Hubbard WA, McElroy GK. Establishment of consistent gait after fitting of new com- 
ponents. J Rehab Res Dev. 1995;32:32-5.

157. Vrieling AH, van Keeken HG, Schoppen T, Otten B, Halbertsma JPK, Hof AL, et al. Gait termination in lower limb amputees. Gait Posture. 2008;27:82-90.

158. van Keeken HG, Vrieling AH, Hof AL, Postema K, Otten B. Controlling horizontal deceleration during gait termination in transfemoral amputees: measurements and simulations. Med Eng Phys. 2012;35:583-90.

159. Jian Y, Winter DA, Ishac MG, Gilchrist L. Trajectory of the body COG and COP during initiation and termination of gait. Gait Posture. 1993;1:9-22.

160. Lynch J, Robertson DGE, editors. Biomechanics of planned gait termination. XXI Congress of International Society of Biomechanics; Taipei, Taiwan. 2007;

161. Hermodsson Y, Ekdahl C, Persson BM, Roxendal G. Standing balance in trans-tibial amputees following vascular disease or trauma: a comparative study with healthy subjects . Prosthet Orthot Int. 1994;18:150-8.

162. Gauthier-Gagnon C, Grise M, Potvin D. Enabling factors related to prosthetic use by people with transtibial and transfemoral amputation. Arch Phys Med Rehabil. 1999;80:706-13.

163. Miller WC, Deathe AB. The influence of balance confidence on social activity after discharge from prosthetic rehabilitation for first lower limb amputation. Prosthet Orthot Int. 2011;35:379-85.

164. Shirota C, Simon AM, Kuiken TA. Transfemoral amputee recovery strategies following trips to their sound and prosthesis sides throughout swing phase. J NeuroEng Rehabil. 2015;12:79.

165. Sheehan RC, Rábago CA, Rylander JH, Dingwell JB, Wilken JM. Use of perturbation-based gait training in a virtual environment to address mediolateral instability in an individual with unilateral transfemoral amputation. Phys Ther. 2016;[Epub ahead of print].

166. Crenshaw JR, Kaufman KR, Grabiner MD. Compensatory-step training of healthy, mobile people with unilateral, transfemoral or knee disarticulation amputations: a potential intervention for trip-related falls. Gait Posture. 2013;38:500-6.

167. Hak L, Houdijk H, Steenbrink F, Mert A, van der Wurff P, Beek PJ, et al. Speeding up or slowing down?: Gait adaptations to preserve gait stability in response to balance perturbations. Gait Posture. 2012;36:260-4.

168. Hak L, van Dieën JH, van der Wurff P, Prins MR, Mert A, Beek PJ, et al. Walking in an unstable environment: strategies used by transtibial amputees to prevent falling during gait. Arch Phys Med Rehabil. 2013;94:2186-93.

169. Beltran EJ, Dingwell JB, Wilken JM. Margin of stability in young adults with traumatic transtibial amputation walking in destabilizing environments. J Biomech. 2014;47:1138-43.

170. Theeven PJR, Hemmen B, Geers RPJ, Smeets RJEM, Brink PRG, Seelen HAM. Influence of advanced prosthetic knee joints on perceived performance and everyday life activity level of low functional persons with a transfemoral amputation or knee disarticulation. J Rehabil Med. 2012;44:454-61.

171. Burnfield JM, Eberly VJ, Gronely JK, Perry J, Yule WJ, Mulroy SJ. Impact of stance phase microprocessor-controlled knee prosthesis on ramp negotiation and community walking function in K2 level transfemoral amputees. Prosthet Orthot Int. 2012;36:95-104.

172. Legro MW, Reiber GD, Smith DG, del Aguila M, Larsen J, Boone D. Prosthetic evaluation questionnaire for persons with lower limb amputation: assessing prosthetic-related quality of life. Arch Phys Med Rehabil. 1998;79:931-8.

173. Resnik L, Borgia M. Reliability of outcome measures for people with lower-limb amputations: distinguishing true change from statistical error. Phys Ther. 2011;91:555-65. 
174. Powell LE, Myers AM. The activities-specific balance confidence (ABC) scale. J Gerontol A Biol Med Sci. 1995;50:M28-34.

175. Miller WC, Deathe AB, Speechley M. Psychometric properties of the activities-specific balance confidence scale among individuals with a lower-limb amputation. Arch Phys Med Rehabil. 2003;84:656-61.

176. Vrieling AH, van Keeken HG, Schoppen T, Otten EH, Halbertsma JPK, Postema K. Obstacle crossing in lower limb amputees. Gait Posture. 2007;26:587-94.

177. Podsiadlo P, Richardson S. The timed "up and go": a test of basic functional mobility for frail elderly persons. J Am Geriatr Soc. 1991;39:142-8.

178. Schoppen T, Boonstra A, Groothoff JW, de Vries J, Göeken LNH, Eisma WH. The time "up and go" test: reliability and validity in persons with unilateral lower limb amputations. Arch Phys Med Rehabil. 1999;80:825-8.

179. Zaino CA, Merchese VG, Westcott SL. Timed up and down stairs test: preliminary reliability and validity of a new measure of functional mobility. Pediatr Phys Ther. 2004;16:90-8.

180. Buell BC, Willingham LL, Allyn KJ, Hafner BJ, Smith DG, (eds). Evaluation of gait style to ascend and descent stairs for lower limb amputees. In: Proceedings of the 11th World Congres of the International Society of Prosthetics and Orthotics. Hong Kong; 2004 Aug 1-6. p. 367.

181. Buell BC, Willingham LL, Allyn KJ, Hafner BJ, Smith DG, (eds). Evaluation of gait style for hill descent for lower limb amputees. In: Proceedings of the 11th World Congres of the International Society of Prosthetics and Orthotics. Hong Kong; 2004 Aug 1-6. p. 53.

182. Taylor MJ, Gunther J. Standardized Walking obstacle course: preliminary reliability and validity of a functional measurement tool. J Rehabil Outcomes Meas. 1998;2:15-25.

183. Bohannon RW. One-leg balance test times. Percept Mot Skills. 1994;78:801-2.

184. Meulenbelt HE, Geertzen JH, Jonkman MF, Dijkstra PU. Skin problems of the stump in lower-limb amputees: 2. Influence on functioning in daily life. Acta Derm Venereol. 2011;91:178-82.

185. Deathe $A B$, Miller WC. The $L$ test of functional mobility: measurement properties of a modified version of the timed "up \& go" test designed for people with lower-limb amputations. Phys Ther. 2005;85:626-35.

186. Parker K, Kirby L, Adderson J, Thompson K. Ambulation of people with lower-limb amputations: relationship between capacity and performance measures. Arch Phys Med Rehabil. 2010;91:543-9.

187. Di Nardo F, Mengarelli A, Maranesi E, Burattini L, Fioretti S. Assessment of the ankle muscle co-contraction during normal gait: a surface electromyography study. J Electromyogr Kinesiol. 2015;25:347-54.

188. Falconer K, Winter DA. Quantitative assesment of co-contraction at the ankle joint in walking. Electromyogr Clin Neurophsyiol. 1985;25:135-49.

189. Frost G, Dowling J, Byson K, Bar-Or O. Cocontraction in three age groups of children during treadmill locomotion. J Electromygr Kinesiol. 1997;7:179-86.

190. Ingraham KA, Fey PA, Simon AM, Hargrove LJ. Assessing the relative contributions of active ankle and knee assistance on walking mechanics of transfemoral amputees using a powered prosthesis. PLoS One. 2016;11:e0147661.

191. Stuurgroep voor Protocollering en Prijssystematiek Prosthesen. Protocol verstrekkingsproces beenprothesen. Werkgroep Amputatie en Prothesiologie; 2010.

192. Rommers GM, Ryall N, Kap A, de Laat F, van der Linde H. The mobility scale for lower limb 
amputees: The SIGAM/WAP mobility scale. Disabil Rehabil. 2007;7:1:10.

193. Malchov C, Fiedler G. Effect of observation on lower limb gait biomechanics: preliminary results. Prosthet Orthot Int. 2015; [Epub ahead of print].

194. Kendell C, Lemaire ED, Kofman J, Dudek N. Gait adaptations of transfemoral prosthesis users across multiple walking tasks. Prosthet Orthot Int. 2016;40:89-95.

195. van Meulen FB, Klaassen B, Held J, Reenalda J, Buurke JH, van Beijnum BJ, et al. Objective evaluation of the quality of movement in daily life after stroke. Front Bioeng Biotechnol. 2016;13:210. 


\section{SUMMARY}

Chapter 1 provided a short overview on what a transfemoral amputation or knee disarticulation is. This was followed by a description of the different types of prosthetic knee units that are available which include non-microprocessor-controlled and microprocessorcontrolled prosthetic knees. Non-microprocessor-controlled prosthetic knees are limitedly able to change the knee damping properties. This is needed to adequately respond to changes in walking speed and negotiate environmental barriers, such as stairs and ramps. The limited ability to change knee damping properties lead to several adaptations in the gait pattern, which were outlined in chapter 1. In contrast to non-microprocessor-controlled prosthetic knees, microprocessor-controlled prosthetic knees, such as the Rheo Knee II, are capable of changing the knee damping properties and are therefore thought to be beneficial for individuals with an amputation. The hypothesis that microprocessor-controlled prosthetic knees are beneficial for individuals with an amputation has been subject of previous research. When looking in the existing literature, it is apparent that there seems to be a mismatch between more objective outcome measures and subjective outcome measures. After hypothesizing why this mismatch occurs, several research questions were postulated, including:

1. What compensations in terms of joint power and work can be seen in the joints of the residual and intact leg of individuals with a transtibial or transfemoral amputation?

2. What are the differences in muscle activation patterns during walking of individuals with a transfemoral amputation or knee disarticulation when compared to individuals without an amputation?

3. What is the influence of the Rheo Knee II on gait adaptations seen during level walking at varying walking speeds, gait initiation, gait termination, and responses to platform perturbations during walking?

4. What is the influence of the Rheo Knee II on prosthesis-related quality of life, functional status, and balance confidence?

The overarching theme of research question 1 and 2 is increasing our understanding of the influence of the amputation on the gait pattern of individuals with a transfemoral amputation or knee disarticulation. One of the possibilities to achieve this, is to systematically collect and combine results of earlier conducted trials. In chapter $\mathbf{2}$, the results of our systematic review and meta-analysis that focused on adaptations strategies of individuals with a transtibial or transfemoral amputation in terms of joint work or joint power are presented. A total of 13 articles were included of which 12 studied individuals with a transtibial amputation and 2 articles studied individuals with a transfemoral amputation. We found that in 
both groups, the hip extensors of the intact leg show increased activity during early stance. In addition, the plantar flexors of the intact leg of individuals with a transfemoral amputation show increased activity at the end of the stance phase. The majority of adaptations seem to be the consequence of the loss of active ankle and foot function of the amputated leg.

Another possibility to increase our understanding of the gait pattern of individuals with a transfemoral amputation or knee disarticulation is through quantifying muscle activation patterns. In chapter 3 , the results of our experiments comparing muscle activation patterns of walking of individuals with an amputation are compared to the muscle activation patterns of non-amputees. At the residual leg we measured muscle activity of the gluteus maximus, gluteus medius, tensor fasciae latae, rectus femoris, vastus lateralis, biceps femoris, semitendinosus and adductor magnus. At the intact leg, we measured the tibialis anterior, gastrocnemius medialis and soleus. We found that individuals with an amputation showed increased co-activation of the ankle dorsal and plantar flexors of the intact leg. It is thought that this co-activation improves joint stability, which is needed because individuals with an amputation heavily rely on their intact leg. In the residual leg, we found activity of almost all upper leg muscles during the stance to swing phase transition. This increased activity is thought to increase the socket fit at the beginning of the swing phase.

Research question 3 aimed to quantify the effect of the Rheo Knee II on gait adaptations during walking. In chapters $\mathbf{4}$ through $\mathbf{8}$ the results of our randomized cross-over trial are presented. For this trial we included participants that were at least one year after amputation, were at least able to walk outdoors, never had a microprocessor-controlled prosthetic knee prescribed before, and had no other musculoskeletal conditions affecting walking ability or stump problems. Participants were measured twice: once with their own non-microprocessor-controlled prosthetic knee and once with the Rheo Knee II. The order in which prosthetic knees were tested was randomized. In both prosthetic knee conditions the low-profile variflex with EVO prosthetic foot was used. A total of ten individuals were included in this trial as well as a control group consisting of ten individuals without an amputation.

As outlined in chapter 1, the distinctive features of a microprocessor-controlled prosthetic knee might not be used to their full extent during level walking at preferred walking, but might be more pronounced at slower or faster walking speed. In chapter 4 the results of our trial studying the added value of a microprocessor-controlled prosthetic knee at preferred walking speed, $70 \%$ preferred walking speed and 115\% preferred walking speed are presented. We calculated spatiotemporal and kinematic data for each walking speed condition. We found, that the use of the Rheo Knee II led to decreased vaulting of the intact ankle at $70 \%$ preferred walking speed. This could be the result of increased prosthetic knee flexion during the swing phase. On this outcome parameter, however, no statistically significant difference was found. On other outcome parameters, no differences were found. The 
effect of a the Rheo Knee II on level walking at different speeds, thus seems to be limited.

Because the activity pattern of individuals with an amputation has been shown to have a large frequency of activity bouts of 1 or 2 minutes in length, the starting and stopping of gait seems to an important aspect of activities of daily living. Therefore we quantified the added value of the Rheo Knee II during gait initiation (chapter 5) and gait termination (chapter 6). We hypothesized that the increased stance stability and early stance prosthetic knee flexion that are associated with the use of a microprocessor-controlled prosthetic knees would enable participants to more efficiently generate the forces needed to initiate gait or absorb the forces that is needed to terminate gait. We therefore calculated the impulses that are generated or absorbed by both the intact and prosthetic leg, the decoupling of the center of mass and center of pressure, and prosthetic knee kinematics during early stance. We found that the use of the Rheo Knee II had no influence on the studied outcome parameters when compared to the use of a non-microprocessor-controlled prosthetic knee. The outcomes of chapter 5 seem to indicate that the ankle is more momentous than the knee to generate the propulsive forces needed to initiate gait. The outcome of chapter 6 , again, indicate the prominent role the ankle has in the absorption of forces that is needed to terminate gait. In addition, while the Rheo Knee II can dissipate energy through early stance prosthetic knee flexion, participants did not use this feature during gait termination. In both prosthetic knee condition, participants kept the prosthetic knee extended throughout the stance phase, thereby minimizing the potential added value the Rheo Knee II might have during gait termination.

Finally we quantified the added value of the Rheo Knee II on responses to platform perturbations during walking. The results of this trial are described in chapter 7. Because of its complexity, this task is to thought to be most distinctive from level walking at preferred walking speed. Individuals walked at their preferred walking speed on an instrumented treadmill that is incorporated in a platform. During the single limb support phase of the prosthetic leg (stance phase perturbation) and during the prosthetic swing phase (swing phase perturbation), the platform was translated in the posterior direction. The primary outcome was the backwards margin of stability (BMoS). When walking with the Rheo Knee II, individuals increased the BMoS in the steps after the stance phase perturbation when compared to the non-microprocessor-controlled prosthetic knee condition. This is mainly achieved by decreasing foot forward placement in the Rheo Knee II condition. On the swing phase perturbations no differences were found. We also compared perturbed to non-perturbed walking to quantify the strategies that individuals use to cope with the balance perturbations. We found that the stance phase perturbation led to an increased BMoS in the Rheo Knee II condition and in the control group of non-amputees when compared to non-perturbed walking. This was not found in the non-microprocessor-controlled prosthetic knee condition. When looking at the strategies to increase the BMoS, the Rheo Knee II enabled participants to use strategies that are also used by non-amputees to increase the BMoS: predominantly a decrease in foot forward placement. Because we were not able to obtain kinetic variables, it 
is difficult to provide an explanation for this finding. We hypothesized that the increased stance stability that is attributed to the Rheo Knee II enabled participants to decrease foot forward placement and step length without increasing the risk of knee buckling.

In chapter 8 we addressed research question 4 and presented the results of our trial investigating the influence of the Rheo Knee II on prosthesis-related quality of life, selfreported balance confidence, and measures of mobility when compared to the use of a non-microprocessor-controlled prosthetic knee. We found that the use of the Rheo Knee II led to an significantly increased score on the Residual Limb Health subscale of the Prosthesis Evaluation Questionnaire. In addition, we found that the use of the Rheo Knee II increased the number of steps that are needed to complete an obstacle course. On other outcome parameters, no differences were found. These results are both in line and in conflict with previous trials. We believe that the fact that we did not provide a gait training program and the fact that we included participants with a relatively high functional level contributed to the ambiguity.

Finally, chapter 9, discussed the main finding of this thesis. Furthermore, this chapter outlines several methodological considerations for performing prosthetic knee research. Finally, the implications and possibilities for future research are described. 


\section{SAMENVATting}

Hoofdstuk 1 startte met het geven van een korte beschrijving over een transfemorale amputatie en een knie disarticulatie. Vervolgens werd er een overzicht gegeven van de verschillende type protheseknieën die verkrijgbaar zijn, waaronder niet-microprocessorgestuurde en microprocessorgestuurde protheseknieën. De niet-microprocessorgestuurde protheseknie is beperkt in staat om de demping van de knie te veranderen. Het veranderen van de kniedemping is van belang om te kunnen lopen op verschillende snelheden en om adequaat met omgevingsbarrières zoals trappen en hellingen om te gaan. Het feit dat nietmicroprocessorgestuurde protheseknieën beperkt in staat zijn de kniedemping te variëren leidt tot specifieke aanpassingen in het looppatroon welke in hoofdstuk 1 beschreven staan. In tegenstelling tot niet-microprocessorgestuurde protheseknieën zijn microprocessorgestuurde protheseknieën, zoals bijvoorbeeld de Rheo Knee II, wel in staat om de kniedemping te variëren. Vanwege de variabele kniedemping wordt verondersteld dat microprocessorgestuurde protheseknieën een meerwaarde hebben voor mensen met een amputatie. Deze veronderstelling is getest in eerder uitgevoerd onderzoek. Als men naar de resultaten van deze onderzoeken kijkt, lijkt er een mismatch te zijn tussen de meer objectieve uitkomstmaten en de meer subjectieve uitkomstmaten. Nadat in hoofdstuk 1 mogelijke verklaringen voor deze mismatch zijn beschreven, zijn er een viertal onderzoeksvragen beschreven:

1. Welke compensaties zijn er zichtbaar rondom de gewrichten van de stomp en het intacte been van mensen met een transtibiale of transfemorale amputatie in termen van joint work en joint power?

2. Wat zijn de verschillen in spieractivatiepatronen tussen mensen met een transfemorale amputatie of een knie disarticulatie en mensen zonder amputatie tijdens het lopen?

3. Wat is de invloed van de Rheo Knee II op aanpassingen in het gangbeeld tijdens het lopen op verschillende snelheden, het starten van het lopen, het stoppen van het lopen en op reacties op platform perturbaties tijdens het lopen?

4. Wat is de invloed van de Rheo Knee II op de kwaliteit van leven, vertrouwen in de balans en functionele status?

Het overkoepelende thema van onderzoeksvraag 1 en 2 is het beter begrijpen van de invloed van een amputatie op het looppatroon van mensen met een transfemorale amputatie of knie disarticulatie. Eén van de mogelijkheden om dit te bereiken is het systematisch verzamelen en combineren van resultaten uit eerder onderzoek. In hoofdstuk $\mathbf{2}$ staan de resultaten van onze systematische review en meta-analyse gepresenteerd. Deze is gericht op het in kaart brengen van de adaptatie strategieën in termen van joint work of joint power van mensen met een transtibiale of transfemorale amputatie. In deze review werden 
13 artikelen geïncludeerd waarvan er 12 resultaten presenteren van mensen met een transtibiale amputatie en 2 studies resultaten presenteren van mensen met een transfemorale amputatie. De resultaten lieten zien dat in beide groepen de heup extensoren een toename in activiteit laten zien tijdens het begin van de standfase. Daarnaast zijn de plantair flexoren van het intacte been van mensen met een transfemorale amputatie actiever aan het eind van de standfase. De meerderheid van de adaptaties lijken het gevolg te zijn van het verlies van de actieve functie van de enkel en de voet van het geamputeerde been.

Een andere mogelijkheid om ons begrip over het looppatroon van mensen met een transfemorale amputatie of knie disarticulatie te vergroten is het bestuderen van de spieractivatiepatronen tijdens het lopen. Hoofdstuk $\mathbf{3}$ presenteert de resultaten van onze experimenten waarin we de spieractivatiepatronen van mensen met een transfemorale amputatie of knie disarticulatie vergelijken met spieractivatiepatronen van mensen zonder amputatie. We hebben de volgende spieren van de stomp gemeten: de gluteus maximus, gluteus medius, tensor fasciae latae, rectus femoris, vastus lateralis, biceps femoris, semitendinosus en adductor magnus. In het intacte been zijn de tibialis anterior, gastrocnemius en soleus gemeten. De resultaten lieten een toegenomen co-activatie zien van de dorsaal en plantair flexoren bij mensen met een amputatie. Er wordt verondersteld dat deze co-activatie leidt tot een toename in stabiliteit van de enkel. Dit zou nodig zijn, omdat mensen met een amputatie erg afhankelijk zijn van hun intacte been. In de stomp vonden we activiteit van nagenoeg alle bovenbeenspieren tijdens de overgang van de stand- naar de zwaaifase. Er wordt verondersteld dat door deze spieractiviteit de kokerfit toeneemt aan het begin van de zwaaifase.

Onderzoeksvraag 3 was gericht op het bepalen van het effect van de Rheo Knee II op aanpassingen in het looppatroon. In hoofdstuk $\mathbf{4}$ tot en met $\mathbf{8}$ staan de resultaten van onze gerandomiseerde cross-over trial gepresenteerd. Voor dit onderzoek werden mensen geïncludeerd waarbij de amputatie ten minste één jaar geleden werd uitgevoerd, die in staat waren om buitenshuis te lopen, die nog nooit een microprocessorgestuurde prothese voorgeschreven hadden gekregen en die naast de amputatie geen andere musculoskeletale problemen of stompproblemen hadden die de loopvaardigheid zouden kunnen beïnvloeden. De deelnemers aan het onderzoek werden twee keer gemeten: één keer met hun eigen niet-microprocessorgestuurde protheseknie en één keer met de Rheo Knee II. De volgorde waarin de protheseknieën werden getest is gerandomiseerd. In beide condities werd gebruik gemaakt van de low-profile Variflex with EVO prothesevoet. In totaal hebben tien personen met een amputatie en een controlegroep, ook bestaande uit tien personen, aan het onderzoek meegedaan.

Zoals besproken in hoofdstuk 1 wordt het onderscheidend vermogen van de microprocessor-gestuurde protheseknie waarschijnlijk niet volledig benut tijdens het lopen op voorkeurssnelheid. Dit onderscheidend vermogen komt wellicht beter tot zijn recht bij langzamer of sneller lopen. In hoofdstuk $\mathbf{4}$ staan de resultaten beschreven van onze ex- 
perimenten gericht op de vraag of de Rheo Knee II een meerwaarde heeft ten opzichte van niet-microprocessorgestuurde protheseknieën bij het lopen op voorkeursnelheid, 70\% voorkeurssnelheid en $115 \%$ voorkeurssnelheid. Voor elke loopsnelheid werden spatiotemporele en kinematische variabelen berekend. Voor de $70 \%$ voorkeurssnelheid conditie vonden we dat het gebruik van de Rheo Knee II leidde tot een afname van vaulting van de intacte enkel. Dit kan het gevolg zijn van een toename van flexie van de protheseknie tijdens de zwaaifase. Echter, op deze uitkomstmaat werd geen statistisch significante verschil gevonden. Ook op de andere uitkomstmaten werd geen statistisch significant verschil gevonden. De meerwaarde van de Rheo Knee II tijdens het lopen op verschillende snelheden lijkt dus beperkt te zijn.

Een studie die het activiteitenpatroon van mensen met een amputatie heeft onderzocht liet zien dat er een hoge frequentie is van kortdurende periodes van aaneengesloten activiteit (1 of 2 minuten). Dit betekent dat mensen met een amputatie relatief vaak starten en stoppen met lopen. In hoofdstuk $\mathbf{5}$ staan de resultaten gepresenteerd van onze experimenten om de meerwaarde van de Rheo Knee II te bepalen tijdens het starten van het lopen en in hoofdstuk 6 de resultaten van de experimenten gericht op het stoppen van het lopen. We veronderstelden dat de toename in standsstabiliteit van het prothese been en de flexie van de protheseknie tijdens de vroege standfase er toe zouden leiden dat deelnemers beter in staat waren om de krachten te genereren die nodig zijn om te starten met lopen en de krachten te absorberen die nodig zijn om te stoppen met lopen. Om hier inzicht in te kunnen krijgen werden de impulsen die door het intacte en het prothese been werden gegenereerd of geabsorbeerd, de ontkoppeling van het center of mass en center of pressure, en de flexie van de protheseknie tijdens het begin van de standfase berekend. De resultaten van hoofdstuk 5 lijken te wijzen op het feit dat de enkel een belangrijkere rol heeft dan de knie in het generen van de propulsieve krachten die nodig zijn om te starten met lopen. De resultaten van hoofdstuk 6 wijzen in dezelfde richting: de enkel heeft een prominentere rol in het absorberen van de krachten die nodig zijn om te stoppen met lopen in vergelijking met de knie. Daarnaast lieten de resultaten zien dat het mechanisme waarmee de Rheo Knee II energie kan absorberen (flexie van de protheseknie tijdens de vroeg standfase) niet gebruikt werd door de deelnemers aan het onderzoek. Tijdens het lopen met zowel de Rheo Knee II als de niet-microprocessorgestuurde protheseknie werd de protheseknie geëxtendeerd tijdens de standfase. Hierdoor werd het mogelijk voordeel dat de Rheo Knee II heeft tijdens het stoppen van het lopen geminimaliseerd.

Ten slotte hebben we de meerwaarde van de Rheo Knee II op de reacties op platform perturbaties tijdens het lopen bepaald. De resultaten van deze experimenten staan beschreven in hoofdstuk 7. Vanwege de complexiteit van deze taak lijkt deze het meest verschillend te zijn van lopen op voorkeurssnelheid. De deelnemers liepen in deze experimenten op voorkeurssnelheid op een loopband die is ingebed in een platform. Het platform werd in achterwaartse richting geperturbeerd tijdens verschillende momenten in de gangcyclus: tijdens de enkele standfase van het prothese been (standfase perturbatie) en tijdens de 
zwaaifase van het prothesebeen (zwaaifase perturbatie). De primaire uitkomstmaat was de backwards margin of stability (BMoS). De deelnemers lieten een vergrote BMoS van de stappen na de standfase perturbatie zien in de Rheo Knee II conditie. Een vergrote BMoS wordt geassocieerd met een verminderd val risico. De toename in de BMoS was vooral het gevolg van een afname van het naar voren plaatsen zowel het prothese als het intacte been. Er werden geen verschillen gevonden voor de zwaaifase perturbatie. Om inzicht te krijgen in de wijze waarop mensen op de perturbaties reageren hebben we het lopen tijdens de platform perturbaties ook vergeleken met het lopen zonder platform perturbaties. In deze vergelijking vonden we dat de BMoS van de stappen na de standfase perturbatie significant groter was in vergelijking met de BMoS van het lopen zonder perturbaties voor zowel de Rheo Knee II conditie als voor de mensen zonder amputatie. Deze verschillen werden niet gevonden voor de niet-microprocessorgestuurde protheseknie. In deze conditie was de BMoS van de stappen na de standfase pertubatie vergelijkbaar met de BMoS van het lopen zonder perturbaties. Daarnaast vonden we dat de Rheo Knee II de deelnemers in staat stelde om dezelfde strategieën te gebruiken om de BMoS te vergroten die ook gebruikt worden door mensen zonder amputatie. De belangrijkste strategie is het verminderen van het naar voren plaatsen van zowel het intacte als het prothese been. Het is lastig om een verklaring te geven voor de gevonden verschillen, omdat we niet in staat waren om kinetische variabelen te verzamelen. We veronderstellen dat een toename in stabiliteit tijdens de vroege standfase deelnemers in staat stelde om het prothese been minder ver naar voren te plaatsen zonder daarbij het risico te lopen om te vallen.

In hoofdstuk 8 is ingegaan op onderzoeksvraag 4. Dit hoofdstuk presenteert de resultaten van onze experimenten waarin de invloed van de Rheo Knee II op de aan de prothese gerelateerde kwaliteit van leven, zelf-gerapporteerde vertrouwen in de balans en mobiliteitsmaten bepaald is. Wij vonden dat het gebruik van de Rheo Knee II leidde tot een significant toegenomen score op de subschaal Stompgezondheid van de Prosthesis Evaluation Questionnaire. Daarnaast vonden we ook dat het gebruik van de Rheo Knee II leidde tot een toename in het aantal stappen dat nodig was om een obstakelparcours te voltooien. Op de andere uitkomstmaten werden geen verschillen gevonden. Deze resultaten zijn zowel in lijn als in conflict met de resultaten van eerder uitgevoerd onderzoek. Wij zijn van mening dat het feit dat we geen trainingsprogramma hebben gekoppeld aan het gebruik van de Rheo Knee II en het feit dat we mensen hebben geïncludeerd met een veelal hoog functioneel niveau hebben bijgedragen aan de tegenstellingen die bestaan tussen onze resultaten en de resultaten van eerder uitgevoerd onderzoek.

Ten slotte zijn in hoofdstuk 9 de belangrijkste resultaten van dit proefschrift bediscussieerd. Daarnaast zijn er verschillende methodologische overwegingen met betrekking tot het uitvoeren van onderzoek naar protheseknieën besproken. Dit hoofdstuk is afgesloten met de implicaties van de gevonden resultaten en de mogelijkheden voor vervolgonderzoek. 


\section{DANKWOORD}

Zo, mijn proefschrift is af. Eindelijk tijd om eens terug te kijken op de afgelopen periode. Het eerste wat boven komt drijven is hoe anders mijn 'carrière' is verlopen dan dat ik me na mijn afstuderen als fysiotherapeut had bedacht. Een ieder die mij destijds gezegd had dat ik zou gaan promoveren had ik acuut op laten nemen. Uiteindelijk is dat een loos dreigement natuurlijk, want zo'n dokter ben en word ik niet. Toch is het er van gekomen en dat heb ik zeker niet alleen aan mezelf te danken. Een hele roedel mensen hebben elk op hun eigen wijze bijgedragen aan het feit dat ik hier nu sta. Achtentwintighonderd-en-acht bolletjes opplakken (ik heb het uitgerekend) heb ik helemaal zelf gedaan. Maar bij elke andere stap heeft er wel iemand meegekeken of meegedacht. Omdat ik ongetwijfeld mensen ga vergeten, wil ik beginnen met iedereen te bedanken!

In de eerste plaats wil ik mijn begeleiders bedanken. Marc, dank voor de moed die je hebt gehad om mij aan te stellen op het Rheo project. Je kunt mij lezen en weet me op de juiste momenten te stimuleren, af te remmen en mijn gang te laten gaan wanneer ik verzand in detaillistisch geneuzel. Daarnaast heb ik veel van je geleerd over het schrijven van artikelen en heb ik, denk ik, grotendeels jouw schrijfstijl overgenomen. Hans, ik laat het wellicht niet altijd blijken maar ik heb inhoudelijk veel van je geleerd. In je commentaar op artikelen weet je altijd de juiste snaar te raken. Ik heb inmiddels geleerd je commentaar te verwerken, anders krijg ik van de reviewer nagenoeg hetzelfde commentaar. Ook op strategisch vlak heb $i k$ veel van je geleerd: ik ken weinig mensen die zo in staat zijn in elke groep een ontspannen sfeer te creëren. Bart, hoewel je in een later stadium bij het onderzoek betrokken bent, kwam het voor mij op het juiste moment. Je biomechanische en technische kennis hebben een substantiële bijdrage aan het proefschrift geleverd. Daarnaast bezit je de gave om na een lange discussie de kern in één zin te kunnen samenvatten. Ook een speciaal woord van dank voor Jaap Buurke. Jaap, ondanks dat je formeel niet in de groep begeleiders zat, heeft het wel zo gevoeld. De wijze waarop jij richting geeft aan het cluster is een voorbeeld voor mij. Dank voor het vertrouwen dat je vanaf het begin af aan in mij hebt gehad en de kansen die je mij geeft om mij verder te ontwikkelen.

Mijn grote dank gaat ook uit naar alle proefpersonen die volledig belangeloos aan het onderzoek hebben meegedaan. Het was een intensief onderzoek met een grote tijdsinvestering terwijl jullie er weinig voor terug hebben gekregen. Dank daarvoor!

I would also like to express my gratitude to the members of my graduation committee that took the effort to review my thesis and be part of my PhD defense.

Furthermore, I'd like to thank the people from Össur who made this research possible and 
were there to answer questions when needed. Your integrity and drive for an objective evaluation of the Rheo Knee II shows that integer commercial research is possible. I would also like to thank you for your patience, when patient inclusion was not running as smooth as we had hoped. In particular, I would like to thank Knut Lechler, Freygardur Thorsteinsson, Peter Slijkhuis and Ása Gudlaug Lúdviksdóttir for their contributions.

Een aantal van de hoofdstukken in dit proefschrift waren niet mogelijk geweest als we geen gebruik hadden kunnen maken van het CAREN platform, van het Center for Augmented Learning and Training van het Nationaal Militair Revalidatiecentrum Aardenburg in Doorn. Ik wil Fred van der Meer, Agali Mert, en, in het bijzonder, Maarten Prins, Roos van der Lint en Tessa Frunt bedanken voor hun bijdrage.

Ik ben ook veel dank verschuldigd aan Jeroen Olsman. Jeroen, als orthopedisch instrumentmaker heb je alle prothese-aanpassingen gedaan. Maar je hebt veel meer gedaan dan dat. Zo zijn we samen regelmatig 'on tour' geweest om prothese-aanpassingen op locatie te doen zodat we ook mensen konden includeren die niet uit Enschede en omgeving kwamen. Daarnaast dank voor het feit dat je ben je gewoon een prettig mens bent.

Ik ben een heel groot woord van dank verschuldigd aan de collega's bij RRD. Het is fijn om in een organisatie met gedreven mensen te werken waarbij een sterk gevoel van saamhorigheid heerst. In vogelvlucht een aantal speciale bedankjes. Jos, bedankt voor de prachtige omslag en de tips voor het binnenwerk. Leendert, dank voor je begrip voor mijn technisch onvermogen. Wendy en Wil, zonder jullie zou ons werk niet mogelijk zijn. Simone, dank dat je me al die jaren als kamergenoot 'verdragen' hebt, voor je gezelligheid en humor en het omvormen van mijn $\mathrm{OOO}$ via $\mathrm{BOO}$ en OOB naar een BOB-light. Inger, met je vrolijkheid en humor heb je er voor gezorgd dat ik me snel thuis ging voelen bij RRD. Martin, de RRC wordt volgens mij al een tijdje niet meer bijgehouden, maar dank voor alle goede (en minder goede) grappen, je luisterend oor, je enthousiasme en je gezelligheid tijdens een aantal geslaagde congressen. De overbuurvrouwen, dank voor de kopjes koffie en de ruimte die er altijd is geweest voor een gesprek. Joke, je 'kan-ik-even-binnenkomen-momentjes' zijn altijd geslaagd. Gerda, dank voor het plannen van alle afspraken met Hans, zelfs als dat schier onmogelijk leek. De RRDiner groep, dank voor de vele mooie momenten binnen en buiten het werk en de geslaagde etentjes. De borrelcommissie, een deel van de gezelligheid zit ook in de voorbereiding van de borrel!

Marieke, Thijs en Corien: jullie verdienen een eigen alinea. Marieke, jij bent één van de personen waardoor ik me vanaf het begin af aan bij RRD heb thuis gevoeld. Op bepaalde punten lijken we best op elkaar waardoor het altijd erg prettig is geweest (en nog steeds is) om even met elkaar te sparren over zaken waar je tegenaan loopt. Ik ben ontzettend blij voor je dat je nu zo goed gaat! Thijs, er zijn weinig mensen die mij zo kunnen beledigen 
zoals jij kan. Je humor werkt enorm relativerend en je hebt voor een hoop ontspanning gezorgd de afgelopen jaren (squashen even daargelaten). Daarnaast sta je altijd klaar om te helpen waar je kan. Dank dat je mijn paranimf wil zijn. Corien, ik ben de laatste jaren jouw kamergenoot geweest. Je zegt wel eens gekscherend dat je mij meer ziet dan je familie en volgens mij is dat eigenlijk ook wel zo. Wat dat betreft prijs ik me gelukkig dat ik bij zo'n fijn mens op de kamer zit. Dank voor je luisterend oor, je oneindige begrip voor de bijzondere trekken die ik kan hebben, je opbouwende kritiek op al mijn pennenvruchten en de wijze waarop we samenwerken. Heel fijn dat ook jij mijn paranimf wil zijn.

Ook wil ik mijn overige vrienden bedanken. Debby, een teken van ware vriendschap is doorzien van het werkelijke verhaal, terwijl de rest voor de gek gehouden wordt. Marian, we kennen elkaar door en door. Dank dat je altijd voor me klaar staat. Wieger, dank voor de mooie vakanties samen (Noorwegen staat ook hoog in mijn top 5), alle andere uitjes en je betrokkenheid. Jolienke, dank dat je me begrijpt en dank voor de vele avonden aan de stoof of op het terras. Hester, dank voor je oneindige vrolijkheid en onze goede gesprekken waardoor ik mezelf beter heb leren kennen. Wouter, naast een gedeelde passie voor wijn en eten delen we ook een passie voor mopperen op de mensheid (o.a. de stiltecoupé). Saskia en Margery, het studentenhuis was een tweede familie voor me en nog steeds zijn jullie er altijd. Ruud, ik leer veel van je relativerend vermogen en het is altijd gezellig wanneer je weer in het Twentse bent. De SKBeters, dank voor de vele geslaagde etentjes en ik hoop dat jullie je een beetje koest houden tijdens de promotie.

Ondanks dat jullie meermaals hebben aangegeven dat jullie niet bedankt hoeven te worden omdat jullie niets aan het proefschrift zouden hebben bijgedragen, ga ik dat toch doen. $\mathrm{Ma}$, dank voor je niet aflatende interesse ook al hoorde ik je af en toe bijna hardop denken wat ik toch de hele dag doe op het werk. Ondanks de hobbels die er zijn geweest ben je altijd positief en ik ben best trots dat ik behoorlijk op je lijk. Jasper, als grote broer heb ik altijd een beetje tegen je opgekeken en dat zal ik ook wel blijven doen denk ik. Het feit dat je altijd gelijk wilt hebben (en ik ook), heeft mijn analytisch vermogen behoorlijk gevoed. Ik heb respect voor hoe hard je werkt om je doelen te bereiken. Daarnaast denk ik ook met veel plezier terug aan ons gebrom. Annerieke, een tweeling bestaat vaak uit twee tegenpolen en wij zijn daarin geen uitzondering. Ik weet daarom zeker dat ik zonder jou niet de persoon zou zijn geworden die ik nu ben. Daarnaast kan ik altijd bij je terecht op de momenten dat ik even een duwtje in de rug nodig heb. Ik ben trots op wat je de afgelopen jaren met hard werken bereikt hebt. Jacob, je hebt het als 'koude kant' vaak zwaar te verduren, maar je bent oprecht een aanwinst voor de familie. Dank dat je Annerieke zo gelukkig maakt.

Als laatste wil ik mijn vader bedanken. Pa, je was, bent en blijft voor mij één van de belangrijkste bronnen van inspiratie. Je hebt me geleerd hoe belangrijk het is om altijd het beste uit jezelf te halen. Ik weet hoe trots je geweest zou zijn vandaag. 


\section{ABOUt the AUthor}

Erik Christiaan Prinsen was born on May $15^{\text {th }} 1983$ in Groenlo, the Netherlands. In 2001 he received his high school diploma from the Ulenhof College in Doetinchem. In 2001, he commenced a study in Medical Biology at the Radboud University in Nijmegen were he obtained his propaedeutics in 2002. In the same year he succesfully applied to start a study in Physical Therapy at the Saxion University of Applied Sciences, which he completed in 2006 (cum laude). Afterwards, he started to work as a Physical Therapist at the regional Queen Beatrix hospital in Winterswijk. Besides his work as a physical therapist, he was working as the quality coordinator of the paramedical departments from 2007 to 2009 and as an interim manager of the physical therapy team from 2008 to 2009. Because of a continuous interest in science, Erik started a study in Physiotherapy Science at the Utrecht University in 2008 and obtained his Master degree (cum laude) in 2010. He was appointed to work on the present PhD project by prof. dr. Hans Rietman, dr. Marc Nederhand and dr. Jaap Buurke. He currently holds a post-doc position with Roessingh Research and Development. $\mathrm{He}$ is also chairman of the Dutch Society for Rehabilitation Physiotherapists.

\section{JOURNAL PUBLICATIONS}

- Prinsen EC, Nederhand MJ, Rietman JS, Koopman HFJM. Added value of a user-adaptive prosthetic knee in planned gait initiation: off to a good start? 2015 IEEE Int Conf Rehabil Robot 2015;Aug:31-36.

- Prinsen EC, Nederhand MJ, Olsman J, Rietman JS. Influence of a user-adaptive prosthetic knee on quality of life, balance confidence and measures of mobility: a randomised cross-over trial. Clin Rehab 2015;29:581-91.

- Tenniglo MJ, Nederhand MJ, Prinsen EC, Nene AV, Rietman JS, Buurke JH. Effect of chemodenervation of the rectus femoris muscle in adults with a stiff knee gait due to spastic paresis: a systematic review with a meta-analysis in patients with stroke. Arch Phys Med Rehabil 2014;95:576-87.

- Wentink EC, Schut VGH, Prinsen EC, Rietman JS, Veltink PH. Intention detection of gait initiation using kinematic sensors and EMG in transfemoral amputees. Gait Posture 2014;39:391-6.

- Wentink EC, Prinsen EC, Rietman JS, Veltink PH. Changes in muscle activity patterns during walking between transfemoral prosthetic users and controls. J Neuroeng Rehabil 2013;10:87. 
- van Dijk SB, Takken T, Prinsen EC, Wittink H. Different anthropometric adiposity measures and their association with cardiovascular disease risk factors: a meta-analysis. Neth Heart J 2012;20:208-18.

- Prinsen EC, Nederhand MJ, Rietman JS. Adaptation strategies of the lower extremities of patients with a transtibial or transfemoral amputation during level walking: a systematic review. Arch Phys Med Rehabil 2011;92:1311-25.

\section{CONFERENCE CONTRIBUtions}

- Prinsen E, Hijmans J, Brandenbarg D, Kahraman T, Buurke J, Koopman B, Vrieling A, Rietman $\mathrm{H}$. Restoration of walking ability in the early stages after transfemoral amputation. Preliminary results of an ongoing trial. ISPO World Congress. June 22-25, Lyon, France.

- Prinsen EC, Nederhand MJ, Olsman J, Rietman JS. Gait adaptations of the intact leg in transfemoral amputees. Influence of a microprocessor-controlled prosthetic knee. ISPGR. June 29-July 4 2014, Vancouver, Canada.

- Prinsen EC, Nederhand MJ, Rietman JS. Influence of a microprocessor-controlled prosthetic knee on biomechanics of gait termination: preliminary results. ISPO World Congress. February 4-7 2013, Hyderabad, India.

- Prinsen EC, Nederhand MJ, Rietman JS. Influence of a microprocessor-controlled prosthetic knee on biomechanics of gait initiation: preliminary results. ISPO World Congress. February 4-7 2013, Hyderabad, India.

- Prinsen EC, Nederhand MJ, Rietman JS. Introduction of a new variable to quantify decoupling of the center of mass and center of pressure during gait initiation. ISPGR. June 24-28 2012, Trondheim, Norway.

- Prinsen EC, Nederhand MJ, Rietman JS. Adaptation strategies of the lower extremities of patients with a transtibial or transfemoral amputation during level walking. A systematic review. ESMAC. September 15-17 2011, Vienna, Austria. 


\section{PROGRESS RANGE}

Progress 1: J.W.G.A. Pot, H. Boer, W.H. van Harten, H.J. Hermens, E.R. Seydel, Comprehensive Need-Assessment. Ontwikkeling van een meetinstrument voor zorgbehoeften en kwaliteitsbeoordeling door patiënten, Roessingh Research \& Development, the Netherlands, September 1994, ISBN 90-25452-01-2.

Progress 2: N.G. A. van Leerdam, H.J. Hermens, Revalidatietechnologie in Euregio, Roessingh Research \& Development, the Netherlands, July 1995, ISBN 90-75452-02-0.

Progress 3: L. Duda. L.O. van Noort, S. Röseler, B.O.L. Greitemann, W.H. van Harten, N.S. Klazinga, Rehabilitation in Germany and the Netherlands, A comparison of two rehabilitation systems, Roessingh Research \& Development, the Netherlands, August 1995, ISBN 90-75452-03-9.

Progress 4: Hermie J. Hermens, Anand V. Nenen, Gerald Zilvold, Electrophysiological Kinesiology, Proceedings of the 11 th congress of the International Society of Electrophysiology and Kinesiology in Enschede, The Netherlands 1996, Roessingh Research \& Development, the Netherlands, October 1996, ISBN 90-75452-04-7.

Progress 5: Wim H. van Harten, Bouwen aan een kwaliteitssysteem in de revalidatiezorg. Een poging tot constructieve technology assessment van een kwaliteitssysteem in een gezondheidszorginstelling, Roessingh Research \& Development, the Netherlands, december 1997, ISBN 90-75452-07-1.

Progress 6: Gert Baardman, Maarten J. IJzerman, Design and evaluation of a hybrid orthosis for people with paraplegia, Roessingh Research \& Development, the Netherlands, November 1997, ISBN 90-75452-08-X

Progress 7: Miriam M.R. Hutten, Lumbar Dynamometry: A useful method for assessment of patients with chronic low back pain?, Roessingh Research \& Development, the Netherlands, November 1999, ISBN 90-75452-13-6.

Progress 8: Drs. A. van der Salm, Prof. Dr. W.H. van Harten, Dr. C.G. B. Maathuis, Ketenkwaliteit Cerebrale Parese Zorg. Een beschrijving van de cerebrale parese zorg en mogelijke verbeteringen hierin, Roessingh Research \& Development, the Netherlands, april 2001, ISBN 90-75452-19-5.

Progress 9: Marc J. Nederhand, Muscle activation patterns in post traumatic neck pain, Roessingh Research \& Development, the Netherlands, March 2003, ISBN 90-75452-27-6.

Progress 10: Michiel J.A. Jannink, Usabillity of custom-made orthopaedic shoes in patients with degenerative disorders of the foot, Roessingh Research \& Development, the Netherlands, September 2004, ISBN 90-75452-28-4.

Progress 11: Mariëtte Blokhorst, State-dependent factors and attention in whiplash associated disorder, Roessingh Research \& Development, the Netherlands, January 2005, ISBN 90-365-2111-4. 
Progress 12: Jaap H. Buurke, Walking after stroke co-ordination patterns \& functional recovery, Roessingh Research \& Development, the Netherlands, February 2005, ISBN 90-3652140-8.

Progress 13: Arjan van der Salm, Spasticity reduction using electrical stimulation in the lower limb of spinal cord injury patients, Roessingh Research \& Development, the Netherlands, October 2005, ISBN 90-365-2253-6.

Progress 14: Govert J. Snoek, Patient preferences for reconstructive interventions of the upper limb in tetraplegia, Roessingh Research \& Development, the Netherlands, December 2005, ISBN 90-365-2255-2.

Progress 15: Joke de Kroon, Therapeutic electrical stimulation of the upper extremity in stroke, Roessingh Research \& Development, the Netherlands, December 2005, ISBN 90 365-2269-2.

Progress 16: Van Dijk H. Motor skill learning, age and augmented feedback. Roessingh Research and Development, the Netherlands, March 2006, ISBN 90-365-2302-9.

Progress 17: Mes CAJ. Improving non-optimal results in chronic pain treatment. Roessingh Research and Development, the Netherlands, January 2007, ISBN 90-365-2435-0.

Progress 18: Voerman GE. Musculoskeletal neck-shoulder pain: a new ambulant myofeedback intervention approach. Roessingh Research and Development, the Netherlands, March 2007, ISBN 90-365-2460-1

Progress 19: Kallenberg LAC. Multi-channel array EMG in chronic neck-shoulder pain. Roessingh Research and Development, the Netherlands, March 2007, ISBN 90-365-2459-8

Progress 20: Huis in 't Veld MHA. Work-related neck-shoulder pain: The role of cognitivebehavioural factors and remotely supervised treatment. Roessingh Research and Development, the Netherlands, December 2007, ISBN 978-90-365-2584-8

Progress 21: Judith F.M. Fleuren. Assessment of Spasticity: From EMG to patients' perception. Roessingh Research and Development, the Netherlands, October 2009, ISBN 978-90365-2869-6

Progress 22: Jasper Reenalda. Dynamic sitting to prevent pressure ulcers in spinal cord injured. Roessingh Research and Development, the Netherlands, October 2009, ISBN 97890-365-2884-9

Progress 23: G.B. (Gerdienke) Prange. Rehabilitation Robotics: Stimulating restoration of arm function after stroke. Roessingh Research and Development, the Netherlands, October 2009, ISBN 978-90-365-2901-3

Progress 24: Vos-van der Hulst M. Prognostic factors and underlying mechanisms in chronic low back pain. Roessingh Research and Development, the Netherlands, November 2009, ISBN 978-90-365-2881-8

Progress 25: Anke I.R. Kottink-Hutten. Assessment of a two-channel implantable peroneal nerve stimulator post-stroke. Roessingh Research and Development, the Netherlands, Febuary 2010, ISBN: 978-90-365-2959-4 
Progress 26: Marit G.H. van Weering. Towards a new treatment for chronic low back pain patients. Roessingh Research and Development, the Netherlands, May 2011, ISBN: 978-90365-3180-1

Progress 27: Jitske Gulmans. Crossing Boundaries: Improving Communication in cerebral palsy care. Roessingh Research and Development, the Netherlands, Febuary 2012, ISBN: 978-90-365-3305-8

Progress 28: Birgit Molier. Influence of augmented feedback on learning upper extremity tasks after stroke. Roessingh Research and Development, the Netherlands, March 2012, ISBN: 978-90-365-3296-9

Progress 29: Rosemary Dubbeldam. Towards a better understanding of foot and ankle kinematics in rheumatoid arthritis. Roessingh Reseacht and Development, the Netherlands, October 2012, ISBN: 978-90-365-3407-9

Progress 30: Richard Evering. Ambulatory feeback at daily physical activity patterns. Roessingh Research and Development, the Netherlands, April 2013, ISBN: 978-90-365-3512-0

Progress 31: Malhotra S. Does spasticity interfere with funktional recovery after stroke? Roessingh Research and Development, the Netherlands, November 2013, ISBN: 978-90-3653567-0

Progress 32: Monique Tabak. Telemedicine for patients with COPD. New treatment approches to improve daily activity behaviour. Roessingh Research and Development, the Netherlands, February 2014, ISBN: 978-94-6108-590-0

Progress 33: Hester Trompetter. ACT with pain. Measurement, efficacy and mechanisms of Acceptance \& Commitment Therapy. Roessingh Research and Development, the Netherlands, september 2014, ISBN: 978-90-365-3708-7

Progress 34: Harm op den Akker. Smart Tailoring of Real-Time Physical Activity Coaching Systems. Roessingh Research and Development, the Netherlands, October 2014, ISBN: 97890-365-3762-9

Progress 35: Stephanie Jansen-Kosterink. The added value of telemedicine for physical rehabilitation. Roessingh Research and Development, the Netherlands, December 2014, ISBN: 978-90-823196-0-6

Progress 36: Inge Marie Velstra. Advanced insights in upper limb function of individuals with cervical spinal cord injury. Roessingh Research and Development, the Netherlands, December 2015, ISBN: 978-90-365-3929-6

Progress 37: Marieke Kloosterman. Keep on Rolling. Functional evaluation of powerassisted wheelchair use. Roessingh Research and Development, the Netherlands, June 2016, ISBN: 978-90-365-4120-6. 


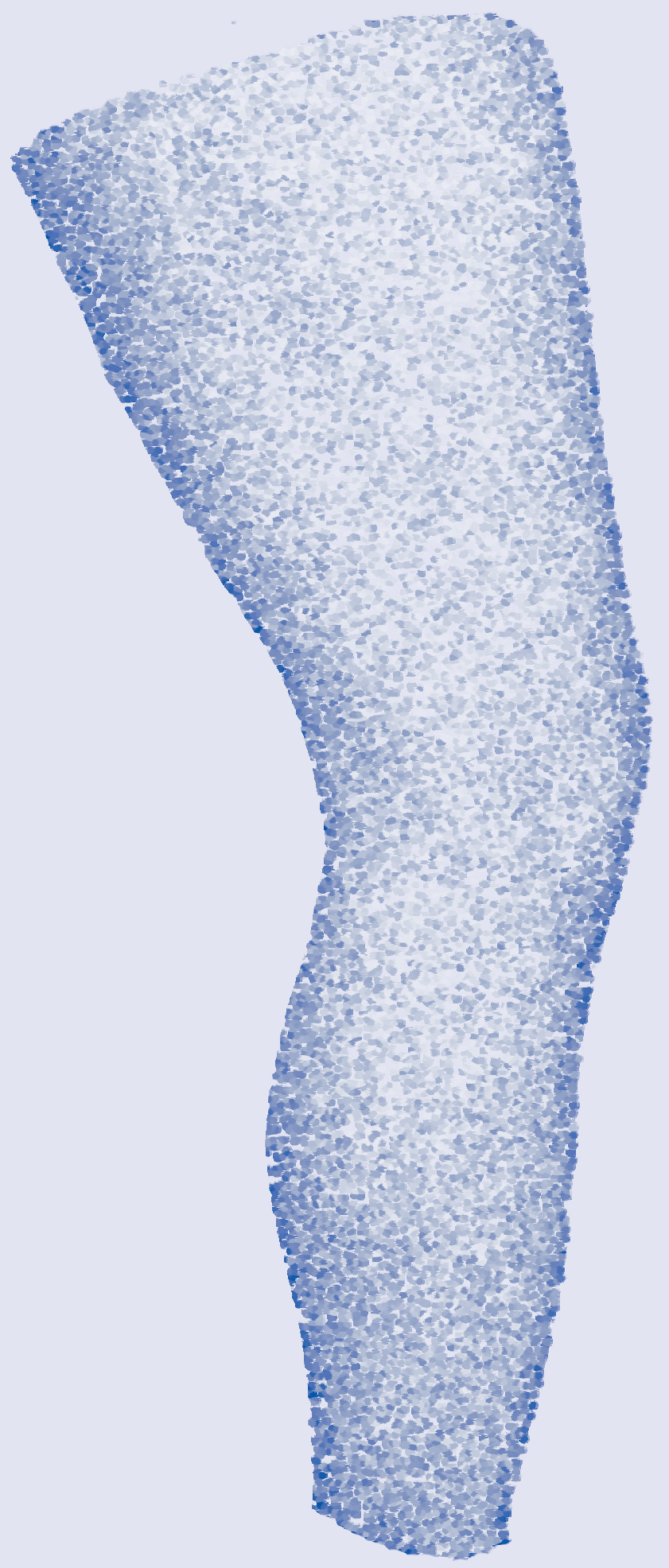

\section{Whyshossingh 38}

ISBN 978-90-365-4206-7 